\title{
A Dichotomy for the Generalized Model Counting Problem for Unions of Conjunctive Queries
}

\author{
Batya Kenig \\ University of Washington
}

\author{
Dan Suciu \\ University of Washington
}

\begin{abstract}
We study the generalized model counting problem, defined as follows: given a database, and a set of deterministic tuples, count the number of subsets of the database that include all deterministic tuples and satisfy the query. This problem is computationally equivalent to the evaluation of the query over a tuple-independent probabilistic database where all tuples have probabilities in $\left\{0, \frac{1}{2}, 1\right\}$. Previous work has established a dichotomy for Unions of Conjunctive Queries (UCQ) when the probabilities are arbitrary rational numbers, showing that, for each query, its complexity is either in polynomial time or \#P-hard. The query is called safe in the first case, and unsafe in the second case. Here, we strengthen the hardness proof, by proving that an unsafe UCQ query remains \#P-hard even if the probabilities are restricted to $\left\{0, \frac{1}{2}, 1\right\}$. This requires a complete redesign of the hardness proof, using new techniques. A related problem is the model counting problem, which asks for the probability of the query when the input probabilities are restricted to $\left\{0, \frac{1}{2}\right\}$. While our result does not extend to model counting for all unsafe UCQs, we prove that model counting is \#P-hard for a class of unsafe queries called Type-I forbidden queries.
\end{abstract}

\section{KEYWORDS}

Model counting, Tuple-Independent Databases, \#P-hardness

\section{ACM Reference Format:}

Batya Kenig and Dan Suciu. 2021. A Dichotomy for the Generalized Model Counting Problem for Unions of Conjunctive Queries. In Proceedings of the 40th ACM SIGMOD-SIGACT-SIGAI Symposium on Principles of Database Systems (PODS '21), June 20-25, 2021, Virtual Event, China. ACM, New York, NY, USA, 51 pages. https://doi.org/10.1145/3452021.3458313

\section{INTRODUCTION}

Fix a First Order (FO) sentence $Q$. The generalized model counting problem for $Q$ is the following: given a database $D B$, and a subset of tuples $D_{1} \subseteq D B$, count the number of models of $Q$ that are subsets of $D B$ and include all tuples in $D_{1}$. In the model counting problem, we set $D_{1}=\emptyset$, and the problem is to count the number of models of $Q$ that are subsets of $D B$.

An equivalent formulation to the generalized model counting problem is to state it as a special case of the query evaluation problem on Tuple-Independent probabilistic Database (TID) [11]. In

Permission to make digital or hard copies of all or part of this work for personal or classroom use is granted without fee provided that copies are not made or distributed for profit or commercial advantage and that copies bear this notice and the full citation on the first page. Copyrights for components of this work owned by others than ACM must be honored. Abstracting with credit is permitted. To copy otherwise, or republish, to post on servers or to redistribute to lists, requires prior specific permission and/or a fee. Request permissions from permissions@acm.org.

PODS '21, fune 20-25, 2021, Virtual Event, China

(C) 2021 Association for Computing Machinery.

ACM ISBN 978-1-4503-8381-3/21/06 . \$ \$15.00

https://doi.org/10.1145/3452021.3458313 that setting, each tuple in the domain has an associated probability, and the problem is to compute the probability that a query $Q$ is true over a possible world obtained by including randomly and independently each tuple in the domain. It is not hard to see that the generalized model counting problem corresponds to restricting the probabilities to $\{0,1 / 2,1\}$, while the model counting problem corresponds to the restricting them to $\{0,1 / 2\}$.

In this paper we will restrict the query $Q$ to be a Union of Conjunctive Queries or, equivalently, to a dual of a UCQ (see below). The following dichotomy is known [4]: either $Q$ is computable in PTIME over any TID, or the query evaluation problem is provably \#P-hard over arbitrary TIDs. In the first case the query is called safe, in the second case it is called unsafe. Moreover, one can decide by static analysis over the expression of the query $Q$ whether it is safe or unsafe. In this paper we strengthen that result by proving a dichotomy theorem for the generalized model counting problem: for any UCQ $Q$, if $Q$ is safe then the generalized model counting problem is in PTIME, and if $Q$ is unsafe then the problem is \#P-hard. The syntactic classification into safe/unsafe queries remains the same as for arbitrary TIDs.

One side of this result is trivial. If the query $Q$ is safe, then it can be evaluated in PTIME over any TID, even if all probabilities are in $\{0,1 / 2,1\}$. This paper is about the other side of the proof: if $Q$ is unsafe, then we show that the query evaluation problem is \#P-hard even if the TID is restricted to have probabilities in $\{0,1 / 2,1\}$. As we explain below, some parts of the hardness proof in [4] (namely Sections 6 and 7) continue to hold even when the probabilities are restricted to $\{0,1 / 2,1\}$, but the most difficult part (Section 8 ) requires an entirely new proof. The reason is that the previous proof [4] relies on multiple distinct probabilities in $(0,1)$, a number that depends on the size of the database. In our paper we develop entirely new proof techniques for this most critical piece of the hardness proof. Before we present the technical material, we comment on several aspects of our contributions.

\subsection{Significance}

If $Q$ is an unsafe query, then the evaluation problem over arbitrary TIDs is \#P-hard. But this problem may become tractable if one restricts the input TID. For example, Amarilli et al. [1] prove that any query $Q$ can be evaluated in PTIME if the input TID has bounded tree-width, and Van den Broeck et al. $[3,5]$ prove that any query in $\mathrm{FO}^{2}$ can be evaluated in PTIME if the TID is symmetric. This leads to a natural question: could the query evaluation problem become easier if we restrict what probabilities can appear in the TID? Our result answers this negatively: query evaluation remains hard even if the probabilities are restricted to $\{0,1 / 2,1\}$. In fact, it remains hard even if the probabilities are restricted to $\{0, c, 1\}$, where $c \in(0,1)$ is any fixed constant. The only property needed in our proof is the following simple fact: 
LEMMA 1.1. Let $f\left(x_{1}, \ldots, x_{n}\right)$ be a multivariate polynomial, not identically 0 , where each variable has degree $\leq 2$. Let $c_{1}, c_{2}, c_{3} \in \mathbb{R}$ be three distinct constants. Then there exists an assignment $\theta$ of the variables $x_{1}, \ldots, x_{n}$ with values in $\left\{c_{1}, c_{2}, c_{3}\right\}$, such that $f[\theta] \neq 0$.

The proof is by induction on $n$ : if $f=g x_{n}^{2}+h x_{n}+k$, where $g, h, k$ are multi-variate polynomials in the other variables, at least one not identically 0 , then there exists an assignment $\theta$ s.t. at least one of $g[\theta], h[\theta], k[\theta]$ is $\neq 0$; since a degree 2 polynomial in $x_{n}$ has at most 2 roots, there exists a "non-root" $c_{i} \in\left\{c_{1}, c_{2}, c_{3}\right\}$, thus $f\left[\theta ; x_{n}:=c_{i}\right] \neq 0$. While our hardness proof is complex, the only probabilities that we need to set in the proof are either 0 or 1 or non-roots of a degree 2 multivariate polynomial, where we use Lemma 1.1. Thus, if $Q$ is unsafe, then it remains \#P-hard even if all probabilities are in $\{0, c, 1\}$ for some fixed $c \in(0,1)$. This ruins any hope of improving query evaluation by restricting the probability values.

\subsection{Generalized- v.s. Standard Model Counting}

Our result states that, if a query is unsafe, then the evaluation problem over TID's with probabilities in $\{0,1 / 2,1\}$ is \#P-hard. But what if the probabilities were restricted to $\{0,1 / 2\}$ ? This corresponds to the model counting problem, and is a natural setting in probabilistic databases, where tuples not present in the database have probability 0 , while those in the database could be associated with probability 1/2. Amarilli and Kimelfeld [2] study precisely this problem and prove a dichotomy for the model counting problem for conjunctive queries without self-joins. We also prove \#P-hardness for the model counting problem, but only for a restricted class of queries called final, type I queries. This complements the result proven by Amarilli and Kimelfeld. We leave open the question whether any unsafe UCQ is hard for model counting. Thus, with the exception of forbidden type I queries, in this paper we study the generalized model counting problem for a query $Q$, which we denote by $\operatorname{GFOMC}(Q)$. We argue next that this is, in fact, a more natural problem than the model counting problem.

\subsection{Dual Queries}

The dual of a First Order query $Q$ is obtained by switching $\exists$ and $\forall$, and switching $\vee$ and $\wedge$. Many problems over Boolean formulas are closed under duals, for example the satisfiability for a class of Boolean formulas is in PTIME iff the validity for the class of duals is also in PTIME; similarly, model counting has the same complexity for a class of Boolean formulas and for its dual. The same property holds for GFOMC. The query evaluation problem for $Q$ on a TID is essentially the same as the evaluation problem of its dual $Q^{\prime}$ on the TID where each probability $p$ is replaced by $p^{\prime} \stackrel{\text { def }}{=} 1-p$, because $\operatorname{Pr}(Q)=1-\operatorname{Pr}^{\prime}\left(Q^{\prime}\right)$. Thus, $\operatorname{GFOMC}(Q)$ and $\operatorname{GFOMC}\left(Q^{\prime}\right)$ have the same complexity. However, duality does not preserve the complexity for model counting: if $Q$ is hard on TIDs with probabilities $\{0,1 / 2\}$, it doesn't follow that is dual $Q^{\prime}$ is also hard on TIDs with probabilities in $\{0,1 / 2\}$. For that reason, we find the generalized model counting problem a more robust notion than the model counting problem, and will focus on it in this paper.

Throughout the paper we will discuss duals of UCQs instead of UCQs. We denote the class of sentences that are duals of UCQs by $\forall C N F$. Since model counting for UCQs correspond to restricting probabilities to $\{0,1 / 2\}$, we define model counting for $\forall C N F$ to mean restricting the probabilities to $\{1 / 2,1\}$.

\subsection{Final Queries}

The hardness proofs in [4] follows a simple high level structure. First, show that if $Q$ is unsafe, then it can be rewritten to a simpler query $Q^{\prime}$, which is still unsafe, such the computation problem for $\operatorname{Pr}\left(Q^{\prime}\right)$ can be reduced in polynomial time to that for $\operatorname{Pr}(Q)$; in particular, hardness of $Q^{\prime}$ implies hardness of $Q$. Second, provide a direct \#P-hardness proof for any unsafe query $Q^{\prime}$ in the simpler class. Usually, the polynomial time reduction consists of adding to the database tuples with probabilities 0 or 1 , never different probability values (see [4, Definition 4.13]). For example, to prove that $\forall x \forall y(R(x) \vee S(x, y) \vee T(y) \vee A(x)) \wedge \forall y B(y)$ is hard it suffices to set all probabilities in $A$ to 0 and all probabilities in $B$ to 1 , and obtain the query $\forall x \forall y(R(x) \vee S(x, y) \vee T(y))$ then prove that the latter is hard. A forbidden query is an unsafe query where no more rewriting to a simpler unsafe query is possible.

The first step of the proof in [4] also applies to GFOMC. Thus, in order to prove that $\operatorname{GFOMC}(Q)$ is hard for any unsafe query $Q$, it suffices to prove it for "forbidden" queries $Q$. The definition of forbidden queries in [4] is quite technical. To prove hardness for Type I queries, we do not need forbidden queries, but instead prove hardness for a slightly larger, and easier to describe class of queries $Q$, called final queries; we will return to forbidden queries in the full version of this paper to prove hardness for Type II queries [7]. All queries considered in this paper are bipartite queries, meaning that they have only two variables, $x, y$, and three kinds of atoms: two unary atoms $R(x), T(y)$, and an arbitrary number of binary atoms $S_{j}(x, y), j=1,2, \ldots$. A type I query contains both atoms $R(x)$ and $T(y)$, and is unsafe iff these two atoms are connected by the clauses of $Q$. If setting any atom to 0 or to 1 makes the query safe, then we call it final; if the query is not final, then we can simply set that atom to 0 or 1 and prove hardness for the simpler query $Q^{\prime}$. Our main result in this paper consists of proving that, for every final query, $\operatorname{GFOMC}(Q)$ is \#P-hard.

\subsection{Reducing \#P2CNF to GFOMC}

A positive $2 \mathrm{CNF}$ formula is $\Phi=\bigwedge_{(i, j) \in E}\left(X_{i} \vee X_{j}\right)$. The model counting problem, denoted \#P2CNF is \#P-hard. Provan and Ball [9] proved that it remains $\# \mathrm{P}$-hard even when the graph $E$ is bipartite, in which case the problem is denoted \#PP2CNF. We use reductions from these problems to prove hardness for $\operatorname{GFOMC}(Q)$.

Our reduction is a polynomial-time reduction, also called Cookreduction (as opposed to the many-one polynomial time reduction, or Karp-reduction). Specifically, we construct a sequence of databases $D B_{1}, D B_{2}, \ldots$ with probabilities in $\{0, c, 1\}$ (i.e., where $c \in$ $(0,1)$ is fixed), and show how to compute \# $\Phi$ from $\operatorname{Pr}_{1}(Q), \operatorname{Pr}_{2}(Q), \ldots$ To compute \#Ф, we need to solve a linear system of equations, and the crux of the hardness proof consists of showing that the matrix of this system is non-singular. We call this matrix the big matrix, since its size is polynomial in the size of $\Phi$.

This is the place where our proof diverges from that in [4]. There, the databases $D B_{1}, D B_{2}, \ldots$ were isomorphic, but used different probabilities, hence the need to use very many distinct probability values. In contrast, we construct databases whose probabilities are 
only among $\{0, c, 1\}$, and, instead, we vary the number of tuples. Each database $D B_{i}$ consists of disjoint paths (formal definition in Sec. 3 ) of lengths that depends on $i$. Instead of varying the probability values in $(0,1)$ as in [4], here we fix the probability values in $\{0, c, 1\}$ and vary the length of the paths.

\subsection{Connecting Logic and Algebra}

We show that the non-singularity of the big matrix follows by proving that a certain "small matrix", $A$, is non-singular. The small matrix is a $2 \times 2$ matrix that describes the probabilities of a single link in the path. Intuitively, when the small matrix is non-singular, then by increasing the path length $i$ in $D B_{i}$ we gain more information about $\# \Phi$ from probability $\operatorname{Pr}(Q)$ on $D B_{i}$. The small matrix depends only on the query $Q$, more precisely, on the arithmetization of its lineage $Y$ on one link of the path. The arithmetization of a Boolean formula $Y$ is a multilinear polynomial $y$ that agrees with $Y$ at all points in $\{0,1\}^{n}$. For example, if $Q=\forall x \forall y(R(x) \vee S(x, y)) \wedge(S(x, y) \vee T(y))$, then the lineage is $Y=(R \vee S) \wedge(S \vee T)$, and its arithmetization is $y(r, s, t)=r t+s-r s t$. Equivalently, the arithmetization is the formula for the probability of $Y$; if $\operatorname{Pr}(R)=\operatorname{Pr}(S)=\operatorname{Pr}(T)=$ $1 / 2$, then $\operatorname{Pr}(Y)=y(1 / 2,1 / 2,1 / 2)=5 / 8$. The small matrix $A$ is $\left(\begin{array}{ll}y[r=0, t=0] & y[r=0, t=1] \\ y[r=1, t=0] & y[r=1, t=1]\end{array}\right)=\left(\begin{array}{cc}s & s \\ s & 1\end{array}\right)$. At its core, our hardness proof relies on the following connection between logic and algebra: the small matrix is non-singular iff the bipartite query $Q$ connects the atoms $R$ and $S$. We state here the formal lemma that captures this connection.

LEMMA 1.2. Let $y$ be the arithmetization of a Boolean formula $Y$, and let $R, T$ be two Boolean variables. Denote the following matrix of polynomials:

$$
\mathbf{y} \stackrel{\operatorname{def}}{=}\left[\begin{array}{ll}
y_{00} & y_{01} \\
y_{10} & y_{11}
\end{array}\right]
$$

where $y_{00} \stackrel{\text { def }}{=} y[r:=0 ; t:=0]$ is obtained by setting $r, t$ to 0 , and similarly for $y_{01}, y_{10}, y_{11}$. Then the following are equivalent: (1) $Y$ disconnects $R, T$, i.e. $Y \equiv F \wedge G$ such that $R \in \operatorname{Vars}(F), T \in$ $\operatorname{Vars}(G), \operatorname{Vars}(F) \cap \operatorname{Vars}(G)=\emptyset$. (2) $\operatorname{det}(\mathbf{y}) \equiv 0$.

The direction (1) $\Rightarrow(2)$ is immediate, because $y$ factorizes as $y=f \cdot g$ where $r$ occurs only in $f$ and $t$ only in $g$, thus $y_{i j}=f_{i} \cdot g_{j}$ and it follows that $\operatorname{det}(\mathbf{y}) \equiv 0$. For $(2) \Rightarrow(1)$ assume that $y_{00} \cdot y_{11} \equiv$ $y_{01} \cdot y_{10}$. Assume $^{1}$ w.l.o.g. that $\operatorname{gcd}\left(y_{00}, y_{01}, y_{10}, y_{11}\right)=1$, which also implies $\operatorname{gcd}\left(y_{00}, y_{01}, y_{10}\right)=1$ because $y_{00}$ is a multi-linear polynomial $^{2}$. Then define $f_{i} \stackrel{\text { def }}{=} \operatorname{gcd}\left(y_{i 0}, y_{i 1}\right)$ and $g_{j} \stackrel{\text { def }}{=} \operatorname{gcd}\left(y_{0 j}, y_{1 j}\right)$, and we have $y_{i j}=f_{i} \cdot g_{j}$ for all $i, j=0,1$, because $\operatorname{gcd}\left(f_{0}, g_{0}\right)=$ $\operatorname{gcd}\left(y_{00}, y_{01}, y_{10}\right)=1$, and similarly for $\operatorname{gcd}\left(f_{i}, g_{j}\right)=1^{3}$. The claim follows from $y=(1-r)(1-t) y_{00}+(1-r) t y_{01}+r(1-t) y_{10}+r t y_{11}=$ $\left((1-r) f_{0}+r f_{1}\right)\left((1-t) g_{0}+t g_{1}\right) \stackrel{\text { def }}{=} f \cdot g$, which implies condition

${ }^{1}$ If $h \stackrel{\text { def }}{=} \operatorname{gcd}\left(y_{00}, y_{01}, y_{10}, y_{11}\right) \neq 1$, then we define $y_{i j}^{\prime} \stackrel{\text { def }}{=} y_{i j} / h$, obtain $y_{i j}^{\prime}=f_{i}^{\prime} \cdot g_{j}$, and define $f_{i} \stackrel{\text { def }}{=} f_{i}^{\prime} h$.

${ }^{2}$ Assuming $p=\operatorname{gcd}\left(y_{00}, y_{01}, y_{10}\right)$ then $p^{2} \mid y_{00} \cdot y_{11} \equiv y_{01} \cdot y_{10}$, but $p \wedge y_{11}$, implying $p^{2} \mid y_{00}$, which is impossible since $y_{00}$ is multilinear.

${ }^{3}$ To see this, consider $y_{00}$, and let $p$ be an irreducible factor of $y_{00}$ (i.e., $y_{00}=y_{00}^{\prime} p$ ). Therefore, since $y_{00} y_{11} \equiv y_{01} y_{10}$ then $p \mid y_{01}$ or $p \mid y_{10}$. If it is the former, then $p \in$ $\operatorname{gcd}\left(y_{00}, y_{01}\right)=f_{0}$, and if the latter then $p \in \operatorname{gcd}\left(y_{00}, y_{10}\right)=g_{0}$. Hence, $y_{00} \equiv f_{0} g_{0}$.
(1) by defining $F, G$ the Boolean formulas associated to $f, g$ respectively.

Finally, our proof falls into place by the following argument. Let $Q$ be a bipartite query. If $Q$ connects the atoms $R(x), T(y)$, then its lineage $Y$ is connected, and thus $\operatorname{det}(\mathbf{y}) \not \equiv 0$. By Lemma 1.1, there exist probability values in $\{0, c, 1\}$ that ensures that the small matrix $A$ is non-singular. Then, the big matrix is non-singular, and we can compute in polynomial time \# $\Phi$ from the probabilities $\operatorname{Pr}_{1}(Q), \operatorname{Pr}_{2}(Q), \ldots$, completing the reduction.

\subsection{Type II Queries}

When a bipartite query $Q$ contains the atoms $R(x), T(y)$, then we call it a type I query. Otherwise, we call it a type II query. Our discussion so far has been restricted to type I queries; we prove their hardness in the main body of the paper. We discuss type II queries, and prove their hardness in the full version of this paper [7]. The proof for Type II queries is more complex than for Type I queries. While the two proofs share many common techniques, they are sufficiently distinct to justify a completely separate proof for Type II queries. In particular, the proof for type I queries is by reduction from $\# \mathrm{P} 2 \mathrm{CNF}$, and that for type II queries by reduction from \#PP2CNF.

\subsection{Organization}

In Section 2 we provide background on final queries, and formally state the problem and main result. We prove the hardness for queries of type I in Section 3, and defer the proof for type II queries to the full version of this paper [7].

\section{PROBLEM STATEMENT}

Fix a finite domain Dom and let Tup(Dom) denote the set of ground tuples consisting of relation names from some fixed vocabulary, and constants from Dom. With some abuse, we write Tup instead of Tup(Dom) when the domain is clear from the context.

A tuple-indepepndent probabilistic database is a pair $\Delta=($ Dom, $p)$ where $p:$ Tup $\rightarrow[0,1]$ associates a probability to each tuple. With some abuse, we simply say that $\Delta$ is a probabilistic database. Given a Boolean query $Q$, its probability is defined as $\operatorname{Pr}(Q) \stackrel{\text { def }}{=}$ $\sum_{W \subseteq \text { Tup: } W \models Q} \operatorname{Pr}(W)$, where the probability of a world $W$ is $\operatorname{Pr}(W) \stackrel{\text { def }}{=}$ $\prod_{t \in W} p(t) \times \prod_{t \in \text { Tup }-W}(1-p(t))$. The probabilistic query evaluation problem, $\mathrm{PQE}(Q)$, is the following: given a probabilistic database (Dom, $p$ ), where $p$ takes rational values, compute $\operatorname{Pr}(Q)$. The following dichotomy theorem was shown in [4]:

THEOREM 2.1. [4] Let $Q$ be any UCQ, then one of the following holds: either $\mathrm{PQE}(Q)$ is in PTIME, or $\mathrm{PQE}(Q)$ is \#P-hard. Moreover, there exists a syntactic condition on the query $Q$ called safety such that $\mathrm{PQE}(Q)$ is in PTIME when $Q$ is safe, and is \#P-hard when $Q$ is unsafe.

For the purpose of this paper we do not need the general definition of safety, and will omit it, except for the special case of bipartite queries defined below. If $Q$ is not safe, then we call it unsafe.

The generalized model counting problem for $Q$, denote $\operatorname{GFOMC}(Q)$, is the following restriction: given a probabilistic database (Dom, $p$ ) 
where $p(t) \in\{0,1 / 2,1\}$ for every tuple $t$, compute $\operatorname{Pr}(Q)$. Obviously, if $\operatorname{PQE}(Q)$ is in PTIME, then so is $\operatorname{GFOMC}(Q)$. We prove that the converse holds too:

Theorem 2.2. For any unsafe $\operatorname{UCQ} Q, \operatorname{GFOMC}(Q)$ is \#P-hard. This result continues to hold even if the probability $1 / 2$ is replaced by some constant probability $c \in(0,1)$.

The high level structure of the proof is similar to that in [4]. Starting with an unsafe query $Q$, first simplify it using simple rewrite rules, as long as the query is still unsafe, until one reaches an unsafe query where every further simplification is safe. Second, prove that each such simplified query $Q$ (called in [4] a forbidden query), $\operatorname{GFOMC}(Q)$ is \#P-hard. The first part of the proof is identical to [4]; we will only state the main result in Theorem 2.5 below, and refer the reader to [4] for the proof. The second part is novel and will be presented in the rest of the paper.

In this paper we will prove Theorem 2.2 for the duals of UCQs, and denote their class $\forall C N F$. We briefly review $\forall C N F$ here. A clause is a disjunction of atoms, $C \equiv R_{1}\left(\mathbf{x}_{1}\right) \vee R_{2}\left(\mathbf{x}_{2}\right) \vee \cdots$; we note that this is the dual notion of a conjunctive query. A homomorphism $C \rightarrow C^{\prime}$ is a function $\operatorname{Vars}(C) \rightarrow \operatorname{Vars}\left(C^{\prime}\right)$ that maps every atom in $C$ to an atom in $C^{\prime}$. We say that $C$ is minimized if every homomorphism $C \rightarrow C$ is a bijection. It is known that every clause is equivalent to a minimized clause ${ }^{4}$. A $\forall \mathrm{CNF}$ formula is a conjunction of clauses, $Q \equiv C_{1} \wedge C_{2} \wedge \cdots$; this is the dual of a UCQ. If there exists a homomorphism $C_{i} \rightarrow C_{j}$ then $C_{j}$ is redundant, and can be removed from $Q$. In this paper we always assume, unless otherwise stated, that all clauses are minimized, and there is no redundant clause. All logical variables are universally quantified, and we will freely switch between prenex normal form of the entire sentence, or of each clause individually, e.g. $\forall x \forall y\left(C_{1} \wedge C_{2}\right)$ v.s. $\forall x \forall y C_{1} \wedge \forall x \forall y C_{2}$.

The lineage of a $\forall C N F$ query $Q$ on a tuple independent database $\Delta=($ Dom,$p)$ is the propositional formula $\Phi_{\Delta}(Q)$ computed as usual, by induction ${ }^{5}$ on the structure of $Q$. We assume that each tuple in the domain Dom is associated with a Boolean variable, thus $\Phi_{\Delta}(Q)$ is a Boolean function over the tuples. We remark that $\Phi_{\Delta}(Q)$ is a Boolean CNF whose size is polynomial in the size of the domain Dom.

We consider a restricted vocabulary consisting of two unary symbols $R, T$, and one or more binary symbols $S_{1}, S_{2}, \ldots$ We call a probabilistic database $\Delta=($ Dom, $p)$ bipartite if Dom is the disjoint union Dom $=U \cup V$, and every tuple $t$ with probability $p(t) \neq 1$ is either $t=R(u)$ or $t=S_{j}(u, v)$ or $t=T(v)$ for $u \in U$ and $v \in V$. We denote by GFOMC $\mathrm{bi}(Q)$ the GFOMC problem where the probabilistic database is restricted to be bipartite.

We define next a bipartite query. It has only two variables $x, y$, its atoms are restricted to be $R(x)$, or $T(y)$, or $S_{j}(x, y)$ for some $j=1,2, \ldots$ Denote by $S_{J}(x, y) \stackrel{\text { def }}{=} \bigvee_{j \in J} S_{j}(x, y)$.

Definition 2.3. We define the following types of sentences:

${ }^{4}$ This follows from the same property for conjunctive queries.

$$
\begin{gathered}
\Phi_{\Delta}(\forall x Q) \stackrel{\text { def }}{=} \bigwedge_{u \in \text { Dom }} \Phi_{\Delta}(Q[u / x]) \\
\Phi_{\Delta}\left(Q_{1} \wedge Q_{2}\right) \stackrel{\text { def }}{=} \Phi_{\Delta}\left(Q_{1}\right) \wedge \Phi_{\Delta}\left(Q_{2}\right) \quad \Phi_{\Delta}\left(Q_{1} \vee Q_{2}\right) \stackrel{\text { def }}{=} \Phi_{\Delta}\left(Q_{1}\right) \vee \Phi_{\Delta}\left(Q_{2}\right)
\end{gathered}
$$

- A left clause of Type $I$ is $\forall x \forall y\left(R(x) \vee S_{J}(x, y)\right)$, where $J \neq \emptyset$.

- A left clause of Type II is $\forall x\left(\bigvee_{\ell=1}^{m}\left(\forall y S_{J_{\ell}}(x, y)\right)\right)$ where $\emptyset \neq$ $J_{1}, \ldots, J_{m} \subseteq[p]$, and $m>1$.

- A middle clause is $\forall x \forall y S_{J}(x, y)$ for $J \neq \emptyset$.

- A right clause of Type $I$ is $\forall y \forall x\left(S_{J}(x, y) \vee T(y)\right)$, where $J \neq \emptyset$.

- A right clause of Type II is $\forall y\left(\bigvee_{\ell=1}^{n}\left(\forall x S_{J_{\ell}}(x, y)\right)\right)$ where $\emptyset \neq$ $J_{1}, \ldots, J_{n} \subseteq[p]$, and $n>1$.

For every $A, B \in\{\mathrm{I}, \mathrm{II}\}$, we define a bipartite query of type $A-B$ to be a $\forall$ CNF query $Q \stackrel{\text { def }}{=} Q_{\text {left }} \wedge Q_{\text {middle }} \wedge Q_{\text {right }}$ where $Q_{\text {left }}$ is a conjunction of left clauses of type $A, Q_{\text {middle }}$ is a conjunction of middle clauses, and $Q_{\text {right }}$ is a conjunction of right clauses of type $B$.

An example of a left clause of type I is $\forall x \forall y\left(R(x) \vee S_{1}(x, y) \vee\right.$ $\left.S_{2}(x, y)\right)$. An example of a left clause of type II is $\forall x\left(\forall y S_{1}(x, y) \vee\right.$ $\left.\forall y S_{2}(x, y)\right)$. For Type II clauses the term "clause" is used with some abuse, since this sentence is not in prenex normal form; the prenex normal form of our example is $\forall x \forall y_{1} \forall y_{2}\left(S_{1}\left(x, y_{1}\right) \vee S_{2}\left(x, y_{2}\right)\right)$.

Definition 4.14 in [4] calls a UCQ query safe if it satisfies a certain syntactic condition. We review below the safety definition only for the special case of bipartite queries and, for the intuition behind this definition, we make two observations. First, let $Q=Q_{\text {left }} \wedge$ $Q_{\text {middle }}$ be a bipartite query without any right clauses. Then one can compute $\operatorname{Pr}(Q)$ in polynomial time on a probabilistic database $\Delta=$ (Dom, $p$ ), in other words $Q$ is safe. Indeed, $Q \equiv \bigwedge_{a \in \operatorname{Dom}} Q[a / x], x$ occurs in each atom of $Q$, hence the events $Q\left[a_{1} / x\right], Q\left[a_{2} / x\right], \ldots$ are independent, and therefore $\operatorname{Pr}(Q)=\prod_{a \in \operatorname{Dom}} \operatorname{Pr}(Q[a / x])$. It is then easy to check that each $\operatorname{Pr}(Q[a / x])$ can be computed in polynomial time. ${ }^{6}$ Second, suppose $Q$ is a bipartite query that can be partitioned into $Q \equiv Q^{\prime} \wedge Q^{\prime \prime}$, where $\operatorname{Symb}\left(Q^{\prime}\right) \cap \operatorname{Symb}\left(Q^{\prime \prime}\right)=\emptyset$, $Q^{\prime}$ has no right clauses, and $Q^{\prime \prime}$ has no left clauses. Then $Q$ is again safe, because $\operatorname{Pr}(Q)=\operatorname{Pr}\left(Q^{\prime}\right) \cdot \operatorname{Pr}\left(Q^{\prime \prime}\right)$. This justifies:

Definition 2.4. A bipartite query is unsafe if it contains a left clause $C_{0}$ and a right clause $C_{k}$ that are connected by a path, i.e. there exists a sequence $C_{0}, C_{1}, \ldots, C_{k}$, called a left-to-right path, such that every two consecutive clauses share an atom. The length of the unsafe query is the minimal $k$ for which there exists a leftto-right path of length $k$.

Let $A, B$ be two problems. A polynomial time many-one reduction from $A$ to $B$, in notation $A \leq_{m}^{P} B$, is a polynomial time computable function $f$ such that, for every instance $x$ of $A, f(x)$ is an instance of $B$ and the answer to problem $A$ on $x$ is the same as the answer to problem $B$ on $f(x)$. A polynomial time reduction from $A$ to $B$, in notation $A \leq P \quad B$, is a polynomial time algorithm for solving instances of $A$ given an oracle for solving instances of $B$. The first part of the proof of Theorem 2.2 is given by the following theorem, where $H_{0} \stackrel{\text { def }}{=} \forall x \forall y(R(x) \vee S(x, y) \vee T(y))$.

THEOREM 2.5. Let $Q$ be a $\forall C N F$ query that is unsafe (according to Definition 4.14 in [4]). Then, either $\mathrm{GFOMC}_{b i}\left(H_{0}\right) \leq_{m}^{P} \mathrm{GFOMC}_{b i}(Q)$, or there exists a bipartite, unsafe query $Q^{\prime}$ such that $\mathrm{GFOMC}_{b i}\left(Q^{\prime}\right) \leq_{m}^{P}$ $\operatorname{GFOMC}_{b i}(Q)$.

Proof. (Sketch) The proof follows directly from [4], more precisely from Theorems 6.3 and 7.3; note that a bipartite query is

${ }^{6}$ It has only unary atoms. 
called a two-leveled query in [4]. The only tool used in those proofs is Lemma 4.17, and it continues to hold if all probabilities are in $\{0,1 / 2,1\}$.

Thus, in order to prove Theorem 2.2, it suffices to show that GFOMC $_{\text {bi }}\left(H_{0}\right)$ is \#P-hard, and $\mathrm{GFOMC}_{\mathrm{bi}}(Q)$ is \#P-hard for any bipartite, unsafe query $Q$. Hardness for $H_{0}$ is already shown in [4], because that proof only uses probabilities in $\{0,1 / 2,1\}$; furthermore, Amarilli and Kimelfeld [2] strengthened this result by showing that model counting for $H_{0}$ is \#P-hard. Thus, we will not consider $H_{0}$ any further in this paper; we only consider bipartite queries.

Next, we need a technical lemma, whose proof is in the full version of the paper [7].

Lemma 2.6. Let $Q$ be a bipartite, unsafe query of type $A-B$, with $A, B \in\{I, I I\}$, and of length $k$. Then there exists a bipartite, unsafe query $Q^{\prime}$ of type $A-A$ of length $\geq 2 k$ such that $\mathrm{GFOMC}_{b i}\left(Q^{\prime}\right) \leq_{m}^{P}$ $\mathrm{GFOMC}_{b i}(Q)$.

We introduce now two simplification rules for queries (these are subsets of the rules in Definition 4.13 [4]). Fix a bipartite query $Q$, and let $S$ be any relational symbol. We denote by $Q[S:=$ false $]$ the query obtained by replacing every occurrence of the atom $S$ by false. Similarly, $Q[S:=$ true] is obtained by replacing $S$ by true. We sometimes abbreviate these rewritings by $Q[S:=0], Q[S:=1]$. As discussed earlier, we always assume that the rewritten query is reduced, by minimizing its clauses and removing redundant clauses.

Lemma 2.7. Let $Q$ be a bipartite query, and let $Q^{\prime}$ be either $Q[S:=$ $0]$ or $Q[S:=1]$. Then (1) $\operatorname{GFOMC}_{b i}\left(Q^{\prime}\right) \leq_{m}^{P} \operatorname{GFOMC}_{b i}(Q)$, (2) $Q$ and $Q^{\prime}$ have the same types (I or II), (3) if $Q^{\prime}$ is unsafe, then so is $Q$ (but the converse does not hold in general), and (4) the length of $Q^{\prime}$ is $\geq$ the length of $Q$.

The proof is immediate and omitted. This justifies:

Definition 2.8. A final query is a bipartite, unsafe query $Q$ with the following property: for any symbol $S$ of $Q$, both $Q[S:=0]$ and $Q[S:=1]$ are safe queries.

Intuitively, if we want to prove that an unsafe query $Q$ is \#P-hard, we can simplify it first to $Q^{\prime}=Q[S:=0]$ or $Q^{\prime}=Q[S:=1]$ and prove that $Q^{\prime}$ is \#P-hard. A final query is one where no further simplifications are possible. A "forbidden query" (Definition 7.2 in [4] and Definition C.10 in the full version of this paper [7]) is defined similarly, but considers some additional simplifications, thus, every forbidden query is final, but the converse does not hold. In this paper we only discuss final queries, but will return to forbidden queries in the full version [7].

We say that $Q$ is disconnected if $Q \equiv Q^{\prime} \wedge Q^{\prime \prime}$, where $Q^{\prime}, Q^{\prime \prime}$ use disjoint sets of symbols. Every final query is connected. Indeed, assuming the contrary, one of $Q^{\prime}, Q^{\prime \prime}$ must must be unsafe, and, assuming $Q^{\prime}$ is unsafe, we can set to true all symbols $S$ occurring in $Q^{\prime \prime}$ and obtain the simpler query $Q^{\prime}$ which is still unsafe, contradicting the fact that $Q$ is final.

Finally, we can now state the main technical result of this paper, which immediately implies Theorem 2.2 . Recall that the model counting problem for UCQ's is defined as $\mathrm{PQE}(Q)$ where the probabilities are restricted to $\{0,1 / 2\}$. Since our discussion is for $\forall C N F$ rather than UCQ's, we define the model counting problem, $\operatorname{FOMC}(Q)$, as $\operatorname{PQE}(Q)$ where the probabilities are restricted to $\{1 / 2,1\}$. In this paper we prove:

THEOREM 2.9. (1) If $Q$ is a final query of type I, then $\mathrm{FOMC}_{b i}(Q)$ is \#P-hard. (2) If $Q$ is a final query of type II of length $\geq 5$, then GFOMC $_{b i}(Q)$ is \#P-hard.

In the rest of the paper we prove Theorem 2.9 (1), and defer part (2) to the full version [7]. We end this section by showing how this theorem proves our main result, Theorem 2.2, which we prove for $\forall C N F$ queries rather than UCQs. Let $Q$ be any unsafe $\forall C N F$ query. By Theorem 2.5, either $\operatorname{GFOMC}_{\mathrm{bi}}\left(H_{0}\right) \leq_{m}^{P} \operatorname{GFOMC}(Q)$, in which case the theorem follows from the fact that $\mathrm{GFOMC}_{\mathrm{bi}}\left(\mathrm{H}_{0}\right)$ is \#P-hard, or $\operatorname{GFOMC}_{\text {bi }}\left(Q^{\prime}\right) \leq_{m}^{P} \operatorname{GFOMC}(Q)$ for some bipartite, unsafe query $Q^{\prime}$ of some type $A-B$. If $A$ is I, then by Lemma 2.6 GFOMC $_{\mathrm{bi}}\left(Q^{\prime \prime}\right) \leq_{m}^{P}$ GFOMC $_{\text {bi }}\left(Q^{\prime}\right)$ for some bipartite, unsafe query $Q^{\prime \prime}$ of type I-I: w.l.o.g. we may assume that $Q^{\prime \prime}$ is final (by Lemma 2.7), then the result follows from Theorem 2.9 (1). If $A$ is II, then we apply Lemma 2.6 three times, to obtain a bipartite, unsafe query $Q^{\prime \prime}$ of type II and of length $\geq 8$, such that GFOMC $\mathrm{bi}_{\mathrm{i}}\left(Q^{\prime \prime}\right) \leq_{m}^{P}$ GFOMC $_{\mathrm{bi}}\left(Q^{\prime}\right)$. As before, we can assume w.l.o.g. that $Q^{\prime \prime}$ is final, hence the result follows from Theorem 2.9 (2).

\section{HARDNESS OF FINAL QUERIES OF TYPE-I}

In this section we prove the first item of Theorem 2.9. A Positive $2 \mathrm{CNF}$, or P2CNF, is a formula $\Phi=\bigwedge_{(i, j) \in E}\left(X_{i} \vee X_{j}\right)$ with $n$ variables and $|E|=m$ clauses. The problem "given a $\operatorname{P} 2 \mathrm{CNF} \Phi$, compute the number of satisfying assignments $\#$ " is denoted $\# \mathrm{P} 2 C N F$ and is known to be \#P-hard. In this section we prove:

Theorem 3.1. For every final query $Q$ of type $I$, \#P2CNF $\leq P$ $\mathrm{FOMC}_{b i}(Q)$

Let $\theta:\left\{X_{1}, \ldots, X_{n}\right\} \rightarrow\{0,1\}^{n}$ be an assignment to $\Phi$ 's variables. We define its signature to be the mapping $\boldsymbol{k}(\theta):\{0,1\}^{n} \rightarrow$ $\{0, \ldots, m\}^{4} \times\{0, \ldots, n\}^{2}$ :

$$
\boldsymbol{k}(\theta) \stackrel{\text { def }}{=}\left(k_{00}(\theta), k_{01}(\theta), k_{10}(\theta), k_{11}(\theta), q_{0}(\theta), q_{1}(\theta)\right)
$$

where

$$
\begin{array}{r}
k_{a b}(\theta) \stackrel{\text { def }}{=}\left|\left\{(i, j) \in E: \theta\left(X_{i}\right)=a, \theta\left(X_{j}\right)=b\right\}\right| \quad a b \in\{0,1\}^{2} \\
q_{a}(\theta) \stackrel{\text { def }}{=}\left|X_{i} \in\left\{X_{1}, \ldots, X_{n}\right\}: \theta\left(X_{i}\right)=a\right| \quad a \in\{0,1\}
\end{array}
$$

Thus, $k_{00}$ is the number of clauses where both $X_{i}, X_{j}$ are false, $k_{11}$ the number of clauses where both are true, and $q_{1}(\theta)$ is the number of true variables $X_{i}$. We assume that $E$ is a directed graph, and contains at most one of $(i, j)$ or $(j, i)$ for all $i \neq j$. Thus, $k_{01}$ and $k_{10}$ may be different, and their sum $k_{01,10} \stackrel{\text { def }}{=} k_{01}+k_{10}$ represents the number of clauses with exactly one variable set to true, and we write $\boldsymbol{k}^{\prime}(\theta) \stackrel{\text { def }}{=}\left(k_{00}, k_{01,10}, k_{11}, q_{0}, q_{1}\right)$ for the undirected signature. For any vector $\boldsymbol{k}=\left(k_{00}, k_{01}, k_{10}, k_{11}, q_{0}, q_{1}\right)$, its count, $\# \boldsymbol{k}$, is the number of assignments $\theta:\left\{X_{1}, \ldots, X_{n}\right\} \rightarrow\{0,1\}^{n}$ with signature $\boldsymbol{k}$, and $\# \boldsymbol{k}^{\prime}$ is the similar undirected counts:

$$
\begin{aligned}
\forall \boldsymbol{k} & \in\{0, \ldots, m\}^{4} \times\{0, \ldots, n\}^{2}: \quad \# \boldsymbol{k} \stackrel{\text { def }}{=}|\{\theta: \boldsymbol{k}(\theta)=\boldsymbol{k}\}| \\
\forall \boldsymbol{k}^{\prime} & \in\{0, \ldots, m\}^{3} \times\{0, \ldots, n\}^{2}: \quad \# \boldsymbol{k}^{\prime} \stackrel{\text { def }}{=}\left|\left\{\theta: \boldsymbol{k}^{\prime}(\theta)=\boldsymbol{k}^{\prime}\right\}\right|
\end{aligned}
$$

Thus, there are $(m+1)^{4}(n+1)^{2}$ counts, and $(m+1)^{3}(n+1)^{2}$ undirected counts, of which at most $(m+1)^{2}(n+1)$ are non-zero, 
because, for any signature, $k_{00}+k_{01,10}+k_{11}=m$ and $q_{0}+q_{1}=n$. To prove Theorem 3.1, we start from a P2CNF $\Phi$ with $m$ clauses, and construct $(m+1)^{2}(n+1)$ bipartite TIDs $\Delta$. Then, we describe an algorithm that, using all probabilities $\operatorname{Pr}_{\Delta}(Q)$, computes all $(m+$ $1)^{2}(n+1)$ consistent undirected counts $\# \mathbf{k}^{\prime}$ (the others are $=0$ ). The reduction from \#P2CNF immediately follows by noting that $\# \Phi=\sum_{\boldsymbol{k}^{\prime}: k_{00}^{\prime}=0} \# \boldsymbol{k}^{\prime}$. This strategy requires computing a polynomial number of counts. The crux of the proof consists in constructing the databases $\Delta$ to make this computation possible. Each such database is a union of blocks; we describe next how to compute $\operatorname{Pr}_{\Delta}(Q)$ when the TID $\Delta$ is a union of blocks. In Section 3.2 we present the reduction from $\# \mathrm{P} 2 \mathrm{CNF}$, and in Section 3.3 we design the blocks such that the answers $\operatorname{Pr}_{\Delta}(Q)$ allow us to compute the counts \#k.

\subsection{The Block TID}

Throughout this section we fix a final query $Q$ of type-I:

$$
Q=\forall x \forall y\left(\bigwedge_{i=1}^{\ell}\left(R(x) \vee S_{J_{i}}(x, y)\right)\right) \wedge C(x, y) \wedge\left(\bigwedge_{k=1}^{r}\left(T(y) \vee S_{J_{k}}(x, y)\right)\right)
$$

Its vocabulary is $\mathcal{R}=\left\{R(x), S_{1}(x, y), \ldots, S_{p}(x, y), T(y)\right\}$. Recall that a bipartite TID $\Delta=($ Dom, $p)$ has a bipartite domain Dom $=$ $U \cup V$.

Definition 3.2. A block $B(u, v)$ is a bipartite TID with two distinguished constants $u, v$. We call $u, v$ the end points of $B(u, v)$, and call any other constant occurring in $B(u, v)$ an internal point.

In what follows, we assume that both end-points $u, v$ of a block are in its left domain, i.e. there are atoms $R(u), R(v)$ but not $T(u), T(v)$ (more precisely, the latter have probabilities 1 ); in the full version of this paper [7] we discuss Type II queries and there we allow $u, v$ to be on the left or right. Two blocks $B(u, v)$ and $B\left(u^{\prime}, v^{\prime}\right)$ are called disjoint if they share at most their end points: $\operatorname{Dom}(B(u, v)) \cap$ $\operatorname{Dom}\left(B\left(u^{\prime}, v^{\prime}\right)\right) \subseteq\{u, v\} \cap\left\{u^{\prime}, v^{\prime}\right\}$. Therefore, when the blocks are disjoint, they can only share tuples $R(u)$ or $R(v)$.

Definition 3.3. We denote by $Y(u, v)$ the lineage of $Q$ over the bipartite $\operatorname{TID} B(u, v): Y(u, v) \stackrel{\text { def }}{=} \Phi_{B(u, v)}(Q)$. For any pair $(a, b) \in$ $\{0,1\}^{2}$ we define

$$
Y_{a b}(u, v) \stackrel{\text { def }}{=} Y(u, v)[R(u):-a, R(v):-b]
$$

In other words, $Y_{a b}$ is the lineage $Y(u, v)$ where we substitute the Boolean variables $R(u), R(v)$ with the values $a, b$ respectively. Using (5), we define:

$$
Y_{a}(u) \stackrel{\text { def }}{=} Y_{a 0}(u, v) \vee Y_{a 1}(u, v)
$$

(we observe that due to monotonicity $Y_{a 0}(u, v) \Rightarrow Y_{a 1}(u, v)$, and hence $Y_{a}(u) \equiv Y_{a 1}(u, v)$. In this paper, we are interested in counting models for the query, and hence the representation of (6)).

THeorem 3.4. Let $U$ be a domain of size n, and let $\Delta=\bigcup_{u, v \in U} B(u, v)$ be a bipartite TID that is a disjoint union of blocks. Assume its bipartite domain is $V_{1} \cup V_{2}$ s.t. $U \subseteq V_{1}$, and that all tuples $\{R(u) \mid u \in U\}$ have probability c. Then:

$$
\underset{\Delta}{\operatorname{Pr}}(Q)=\sum_{\theta: U \rightarrow\{0,1\}} \operatorname{Pr}(\theta) \prod_{u, v \in U} \operatorname{Pr}\left(Y_{\theta(u) \theta(v)}(u, v)\right)
$$

where $\operatorname{Pr}(\theta)=\prod_{u \in U} c^{\theta(u)}(1-c)^{1-\theta(u)}$.
Proof. Since $\Phi_{\Delta}(Q)=\bigwedge_{u, v \in U} Y(u, v)$ and any two Boolean formulas $Y(u, v), Y\left(u^{\prime}, v^{\prime}\right)$ share at most the boolean variables $R(u)$ or $R(v)$ (when $u=u^{\prime}$ or $v=v^{\prime}$ ), we have:

$$
\begin{aligned}
& \operatorname{Pr}(Q)=\operatorname{Pr}\left(\Phi_{\Delta}(Q)\right)=\operatorname{Pr}\left(\bigwedge_{u, v \in U} Y(u, v)\right) \\
= & \sum_{\theta: U \rightarrow\{0,1\}^{n}} \operatorname{Pr}(\theta) \operatorname{Pr}\left(\bigwedge_{u, v \in U} Y(u, v)[R(u):-\theta(u), R(v):-\theta(v)]\right) \\
= & \sum_{\theta: U \rightarrow\{0,1\}^{n}} \operatorname{Pr}(\theta) \operatorname{Pr}\left(\bigwedge_{u, v \in U} Y_{\theta(u) \theta(v)}(u, v)\right) \\
= & \sum_{\theta: U \rightarrow\{0,1\}^{n}} \operatorname{Pr}(\theta) \prod_{u, v \in U} \operatorname{Pr}\left(Y_{\theta(u) \theta(v)}(u, v)\right)
\end{aligned}
$$

where (8) follows because the Boolean functions $Y_{\theta(u) \theta(v)}(u, v)$ and $Y_{\theta\left(u^{\prime}\right) \theta\left(v^{\prime}\right)}\left(u^{\prime}, v^{\prime}\right)$ are over disjoint sets of Boolean variables, and hence they are independent.

Block TID associated with a graph. Let $G(U, E)$ be the undirected graph associated with the P2CNF $\Phi=\bigwedge_{(u, v) \in E}\left(X_{u} \vee X_{v}\right)$, where $n=|U|$ and $m=|E|$. To define the TID, we consider an isomorphic copy of the set $U, U^{\prime}=\left\{u^{\prime} \mid u \in U\right\}$, and define the graph $G^{\prime}\left(U \cup U^{\prime}, E \cup E^{\prime}\right)$, where $E^{\prime}=\left\{\left(u, u^{\prime}\right) \mid u \in U, u^{\prime} \in U^{\prime}\right\}$. That is, $E^{\prime}$ contains, for each node $u \in U$, one edge connecting it to its isomorphic copy $u^{\prime}$ in $U^{\prime}$. We associate every edge $(u, v) \in E$ and $\left(u, u^{\prime}\right) \in E^{\prime}$ with blocks $B(u, v)$ and $B\left(u, u^{\prime}\right)$ respectively, and define the block-disjoint TID $^{7}$ associated with $G$ : $\Delta=\bigcup_{(u, v) \in E} B(u, v) \cup \bigcup_{\left(u, u^{\prime}\right) \in E^{\prime}} B\left(u, u^{\prime}\right)$, where for every nonedge $(a, b) \notin E \cup E^{\prime}$ we define $B(a, b)$ to be the trivial block consisting of all tuples $S_{1}(a, b), \ldots, S_{p}(a, b)$ with probability 1 . In this setting, by (7) we have:

$$
\begin{aligned}
\underset{\Delta}{\operatorname{Pr}}(Q)= & \sum_{\theta: U \rightarrow\{0,1\}^{n}} \operatorname{Pr}(\theta) \prod_{(u, v) \in E \cup E^{\prime}} \operatorname{Pr}\left(Y_{\theta(u) \theta(v)}(u, v)\right) \\
= & \sum_{\theta: U \rightarrow\{0,1\}^{n}} \operatorname{Pr}(\theta) \prod_{(u, v) \in E} \operatorname{Pr}\left(Y_{\theta(u) \theta(v)}(u, v)\right) \\
& \times \prod_{\left(u, u^{\prime}\right) \in E^{\prime}}\left(\operatorname{Pr}\left(Y_{\theta(u) 0}\left(u, u^{\prime}\right)\right)+\operatorname{Pr}\left(Y_{\theta(u) 1}\left(u, u^{\prime}\right)\right)\right) \quad(9) \\
= & \sum_{\theta: U \rightarrow\{0,1\}^{n}} \operatorname{Pr}(\theta) \prod_{(u, v) \in E} \operatorname{Pr}\left(Y_{\theta(u) \theta(v)}(u, v)\right) \prod_{u \in U} \operatorname{Pr}\left(Y_{\theta(u)}(u)\right) \\
= & \sum_{\theta: U \rightarrow\{0,1\}^{n}} \prod_{(u, v) \in E} \operatorname{Pr}\left(Y_{\theta(u) \theta(v)}(u, v)\right) \\
& \times \prod_{u \in U}\left(c \operatorname{Pr}\left(Y_{1}(u)\right)\right)^{\theta(u)}\left((1-c) \operatorname{Pr}\left(Y_{0}(u)\right)\right)^{1-\theta(u)}(11)
\end{aligned}
$$

where the transition from (9) to (10) is by the definition of $Y_{a}(u)$ in (6). The transition from (10) to (11) follows from the fact that $\operatorname{Pr}(\theta)=\prod_{u \in U} c^{\theta(u)}(1-c)^{1-\theta(u)}$.

\subsection{The Reduction from \#P2CNF to $\operatorname{FOMC}(Q)$}

In this section, we show that using an oracle to $\operatorname{FOMC}(Q)$ over a block disjoint TID $\Delta$ allows us to construct a system of linear equations $\mathcal{B}$ whose solution allows us to solve \#P2CNF. We establish three

\footnotetext{
${ }^{7}$ Not to be confused with Block-Disjoint-TIDs that refer to TIDs consisting of disjoint sets of mutual exclusive tuples [11].
} 
conditions on the blocks of $\Delta$, which guarantee that $\mathcal{B}$ is nonsingular, and thus has a unique solution. In section 3.3 we show how to construct the blocks such that these conditions hold.

Fix a final query $Q$ of type-I, and an instance of \#P2CNF $\Phi=$ $\bigwedge_{(i, j) \in E}\left(X_{i} \vee X_{j}\right)$. We let $U=\left\{u_{1}, \ldots, u_{n}\right\}, U^{\prime}=\left\{u_{1}^{\prime}, \ldots, u_{n}^{\prime}\right\}$, $|E|=m$, and $\left.E^{\prime}=\left\{\left(u_{i}, u_{i}^{\prime}\right) \mid u_{i} \in U, u_{i}^{\prime} \in U^{\prime}\right)\right\}$. We create $m$ blocks $B(u, v)$, for all $(u, v) \in E$ and $n$ blocks $B\left(u, u^{\prime}\right)$, for all $\left(u, u^{\prime}\right) \in E^{\prime}$, and define $\Delta=\bigcup_{(u, v) \in E \cup E^{\prime}} B(u, v)$. The blocks will be isomorphic, and therefore, the following quantities do not depend on $u, v$ :

$$
\begin{array}{rr}
y_{a b} \stackrel{\text { def }}{=} \operatorname{Pr}\left(Y_{a b}(u, v)\right) & (u, v) \in E \text { and } a b \in\{0,1\}^{2} \\
y_{a} \stackrel{\text { def }}{=} \operatorname{Pr}\left(Y_{a}(u)\right) & u \in U \text { and } a \in\{0,1\}
\end{array}
$$

A block $B(u, v)$ is symmetric if $\operatorname{Pr}\left(Y_{a b}(u, v)\right)=\operatorname{Pr}\left(Y_{b a}(u, v)\right)$. In our reduction, we construct symmetric blocks, and thus can assume that $y_{a b}=y_{b a}$.

Consider assignment $\theta$ occurring the sum $\sum_{\theta}$ of (11), and let $\boldsymbol{k}(\theta)=\left\{k_{00}(\theta), \ldots, k_{11}(\theta), q_{0}(\theta), q_{1}(\theta)\right\}$ be its signature. The factor $y_{a b}$ occurs precisely $k_{a b}$ times in the product, hence its exponent is $k_{a b}$, i.e. the number of edges $(u, v) \in E$ where $\theta(u)=a$, and $\theta(v)=b$. Likewise, the factor $y_{a}$ occurs precisely $q_{a}$ times in the product, hence its exponent is $q_{a}$, i.e. the number of edges $\left(u, u^{\prime}\right) \in E^{\prime}$ where $\theta(u)=a$. Therefore, (11) becomes:

$$
\begin{gathered}
\operatorname{Pr}_{\Delta}(Q)=\sum_{\substack{k_{00}+k_{01}+k_{10}+k_{11}=m \\
q_{0}+q_{1}=n}} \# \boldsymbol{k}\left(y_{00}\right)^{k_{00}}\left(y_{01}\right)^{k_{01}}\left(y_{10}\right)^{k_{10}}\left(y_{11}\right)^{k_{11}} \\
\cdot\left(c y_{1}\right)^{q_{1}}\left((1-c) y_{0}\right)^{q_{0}}
\end{gathered}
$$

Our unknowns are $\# \boldsymbol{k}$; there is one unknown for every signature $\boldsymbol{k}$. Since the blocks are symmetric, then $y_{01}=y_{10}$, then we may write:

$$
\begin{gathered}
\operatorname{Pr}(Q)=\sum_{\substack{k_{00}+k_{01,10}+k_{11}=m \\
q_{0}+q_{1}=n}} \# \boldsymbol{k}\left(y_{00}\right)^{k_{00}}\left(y_{10}\right)^{k_{01,10}}\left(y_{11}\right)^{k_{11}} \\
\cdot\left(c y_{1}\right)^{q_{1}}\left((1-c) y_{0}\right)^{q_{0}}
\end{gathered}
$$

where $k_{01,10} \stackrel{\text { def }}{=} k_{01}+k_{10}$. Eq. (15) has $(n+1)(m+1)^{2}$ unknowns \# $\boldsymbol{k}$ because $k_{00}+k_{11}+k_{01,10}=m$ and $q_{0}+q_{1}=n$. The coefficient associated with $\# \boldsymbol{k}$ is $\left(y_{00}\right)^{k_{00}}\left(y_{11}\right)^{k_{11}}\left(y_{10}\right)^{k_{01,10}}\left(c y_{1}\right)^{q_{1}}\left((1-c) y_{0}\right)^{q_{0}}$. To construct a system of linear equations that will allow us to solve for the $(n+1)(m+1)^{2}$ unknowns $\# \boldsymbol{k}$, we need to create $(n+1)(m+1)^{2}$ linearly independent equations corresponding to the coefficients.

To that end, we construct $\mathcal{B}$ to be an $(n+1)(m+1)^{2} \times(n+1)(m+1)^{2}$ matrix whose rows correspond to $(n+1)(m+1)^{2}$ distinct, block disjoint TIDs, and whose columns correspond to the $(n+1)(m+1)^{2}$ signatures $\boldsymbol{k}=k_{00}, k_{01,10}, k_{11}, q_{0}, q_{1}$. Every such block-disjointTID is comprised of $m+n$ disjoint blocks, one for every edge in $E \cup E^{\prime}$. Every block corresponding to an edge $(u, v) \in E$ is parameterized by one of $(m+1)^{2}$ values $p \in\left\{1, \ldots,(m+1)^{2}\right\}$, while every block corresponding to an edge $\left(u, u^{\prime}\right) \in E^{\prime}$ is parameterized by one of $(n+1)$ values $q \in\{1, \ldots, n+1\}$ (See Section 3.3). Taking all possible combinations of parameters, we arrive at $(n+1)(m+1)^{2}$ distinct, block-disjoint-TID, one for every combination of parameters in $\left\{1, \ldots,(m+1)^{2}\right\} \times\{1, \ldots, n+1\}$. Likewise, we observe that every coefficient $y_{00}^{k_{00}} y_{11}^{k_{11}} y_{10}^{k_{01,10}}\left(c y_{1}\right)^{q_{1}}\left((1-c) y_{0}\right)^{q_{0}}$ corresponds to a combination of two signatures, one for the edges in $E: \boldsymbol{k}_{1}=\left(k_{00}, k_{01,10}, k_{11}\right)$, and one for the edges in $E^{\prime}: \boldsymbol{k}_{2}=\left(q_{0}, q_{1}\right)$.
From this construction, along with the linear equations specified in (15), we observe that $\mathcal{B}$ is the Kronecker product of two matrices: an $(n+1) \times(n+1)$ matrix denoted $\mathcal{N}$, and an $(m+1)^{2} \times(m+1)^{2}$ matrix denoted $\mathcal{M}$. Hence, showing that $\mathcal{B}$ is non-singular reduces to showing that both $\mathcal{N}$ and $\mathcal{M}$ are non-singular. Since the size of the system $\mathcal{B}$ is polynomial in the size of the data, then this allows us to solve for the counts $\# \boldsymbol{k}$ in PTIME, thus proving hardness.

Proposition 3.5. The $(n+1) \times(n+1)$ system of linear equations $\mathcal{N}$ is invertible.

Proof. Let $t \in\{1, \ldots, n+1\}$ denote $n+1$ distinct, block disjoint TIDs. Each such TID induces a pair of probability values $y_{0}^{(t)}, y_{1}^{(t)}$ (The construction of these TIDs is deferred to Section 3.3). The columns of $\mathcal{N}$ represent the values $q \in\{0, \ldots, n\}$. Therefore, $[\mathcal{N}]_{t q}=\left(y_{1}^{(t)} c\right)^{q}\left((1-c) y_{0}^{(t)}\right)^{n-q}$. Factoring out $\left(y_{0}^{(t)}(1-c)\right)^{n}$ for each row of $\mathcal{N}$, we get $[\mathcal{N}]_{t q}=\left(\frac{c}{1-c} \cdot \frac{y_{1}^{(t)}}{y_{0}^{(t)}}\right)^{q}$. Hence, the quotient of each row $t$ is $\frac{c}{1-c} \cdot \frac{y_{1}^{(t)}}{y_{0}^{(t)}}$. To prove the claim we need to show that $y_{a}^{(t)}>0$ for all $t \geq 1$ and all $a \in\{0,1\}$. Further, we need to show that for every pair $t_{2}>t_{1} \geq 1$ it holds that $\frac{y_{1}^{\left(t_{1}\right)}}{y_{0}^{\left(t_{1}\right)}} \neq \frac{y_{1}^{\left(t_{2}\right)}}{y_{0}^{\left(t_{2}\right)}}$. We prove this claim in Lemma 3.25 in Section 3.3 because it relies on the structural properties of the blocks.

To prove that $\mathcal{M}$ is invertible, we show that it meets three conditions that characterize non-singular matrices. In the rest of this section we present the three conditions on the probabilities $y_{a b}$, and prove that if they are met then the resulting system $\mathcal{M}$ of $(m+1)^{2}$ linear equations is non-singular. In fact, we prove something more general required for proving hardness of Type-II queries.

Let $h \geq 1$ be a natural number. Let $\lambda_{1}, \lambda_{2} \in \mathbb{R}$, and $a_{i}, b_{i}, i=$ $0, \ldots, h$ be real numbers satisfying the following conditions:

$$
\begin{array}{rrr}
\lambda_{1} \neq \pm \lambda_{2} & \text { and } & \lambda_{1} \neq 0, \lambda_{2} \neq 0 \\
b_{i} \neq 0 & \forall i=0, h \\
a_{i} b_{j} \neq a_{j} b_{i} & i \neq j
\end{array}
$$

Let $\mathbf{p}=\left\{p_{1}, \ldots, p_{h}\right\}$ be $h$ natural numbers where $p_{i} \geq 1$. For $i=0, h$ we define:

$$
y_{i}(\mathbf{p})=\prod_{j=1}^{h}\left(a_{i} \lambda_{1}^{p_{j}}+b_{i} \lambda_{2}^{p_{j}}\right) \quad \forall i \in\{0, \ldots, h\}
$$

Finally, define the following $(m+1)^{h} \times(m+1)^{h}$ matrix $\mathcal{M}$ :

$$
\begin{aligned}
& \mathcal{M}_{\mathrm{p}, \mathrm{k}} \stackrel{\text { def }}{=} \prod_{i=0, h} y_{i}^{k_{i}}(\mathbf{p}), \quad \mathbf{p} \in\{1,2, \ldots, m+1\}^{h} \\
& \mathbf{k} \in\{0,1, \ldots, m\}^{h} \quad k_{0}+\ldots+k_{h}=m
\end{aligned}
$$

Example 3.6. For a simple example, assume $h=2, m=2$, then:

$$
\begin{aligned}
& y_{i}\left(p_{1}, p_{2}\right)=\left(a_{1} \lambda_{1}^{p_{1}}+b_{1} \lambda_{2}^{p_{1}}\right)\left(a_{1} \lambda_{1}^{p_{2}}+b_{1} \lambda_{2}^{p_{2}}\right) \quad i=0,1,2 \\
& \mathcal{M}=\left[\begin{array}{cccc}
y_{0}^{2}(1,1) & y_{0}(1,1) y_{1}(1,1) & \ldots & y_{2}^{2}(1,1) \\
y_{0}^{2}(1,2) & y_{0}(1,2) y_{1}(1,2) & \ldots & y_{2}^{2}(1,2) \\
& \ldots & & \\
y_{0}^{2}(3,3) & y_{0}(3,3) y_{1}(3,3) & \ldots & y_{2}^{2}(3,3)
\end{array}\right]
\end{aligned}
$$


Each row of $\mathcal{M}$ has all products $y_{0}^{k_{0}} y_{1}^{k_{1}} y_{2}^{k_{2}}$ where $k_{0}+k_{1}+k_{2}=2$.

Theorem 3.7. The matrix $\mathcal{M}$ is non-singular

Before we prove the theorem, we show how to use it to prove Theorem 3.1. We set $h=2$. Given the P2CNF formula defined by the graph $(U, E)$, we will construct a TID obtained as a disjoint union of blocks $\Delta=\bigcup_{(u, v) \in E} B(u, v)$. The probability of $Q$ is given by Eq. (15). Fix two numbers $p_{1}, p_{2} \geq 1$. We will describe in the next section how to construct a symmetric block $B(u, v)$ such that its probabilites are given by expresions similar to (19), more precisely:

$$
\begin{aligned}
& y_{00}=\prod_{j=1,2}\left(a_{00} \lambda_{1}^{p_{j}}+b_{00} \lambda_{2}^{p_{j}}\right) \\
& y_{10}=\prod_{j=1,2}\left(a_{10} \lambda_{1}^{p_{j}}+b_{10} \lambda_{2}^{p_{j}}\right) \\
& y_{11}=\prod_{j=1,2}\left(a_{11} \lambda_{1}^{p_{j}}+b_{11} \lambda_{2}^{p_{j}}\right)
\end{aligned}
$$

where the coefficients satisfy conditions (16)-(18). We use repeatedly the oracle for $\operatorname{Pr}(\Delta)$, once for each $(m+1)^{2}$ combination of values $p_{1}, p_{2} \in\{1,2, \ldots, m+1\}$, and obtain a system of linear equations with unknowns $\# \mathbf{k}^{\prime}$. By Theorem 3.7 this system has a unique solution, which can be computed in polynomial time using Gaussian elimination. This gives us all the undirected counts $\# \mathbf{k}^{\prime}$, from which we extract $\# \Phi=\sum_{\# \mathbf{k}^{\prime}: k_{00}=0} \# \mathbf{k}^{\prime}$.

In the rest of this section we prove Theorem 3.7, by proving a series of lemmas in calculus, of possible independent interest.

Lemma 3.8. For each $\mathbf{k}=\left(k_{1}, \ldots, k_{h}\right) \in\{0, \ldots, m\}^{h}$, define the following polynomial in variables $\mathbf{y}=\left(y_{1}, \ldots, y_{h}\right): g_{\mathbf{k}}(\mathbf{y}) \stackrel{\text { def }}{=} y_{1}^{k_{1}} \cdots y_{h}^{k_{h}}$. Then, the polynomials $g_{\mathbf{k}}, \mathbf{k} \in\{0, \ldots, m\}^{h}$ are linearly independent.

Proof. Assume the contrary, that there exist coefficients $a_{k_{1}, \ldots, k_{h}}$ such that, denoting $f(\mathbf{y}) \stackrel{\text { def }}{=} \sum_{\mathbf{k}} a_{\mathbf{k}} \prod_{i} y_{i}^{k_{i}}$, the polynomial $f$ is identically $0, f \equiv 0$. For each $i=1, h$, let $S_{i} \stackrel{\text { def }}{=}\left\{v_{i, 0}, \ldots, v_{i, m}\right\}$ be a set of $m+1$ distinct values, and denote by $A_{i}$ the Vandermonde matrix defined by the set $S_{i}:\left(A_{i}\right)_{k \ell}=v_{i, \ell}^{k}$, for $0 \leq k, \ell \leq m$. By our assumption that $f \equiv 0$, then for any combination of values $\left(u_{1}, \ldots, u_{h}\right) \in S_{1} \times \cdots \times S_{h}$ we have:

$$
\sum_{\mathbf{k}} a_{\mathbf{k}} \prod_{i} u_{i}^{k_{i}}=0
$$

By using all $(m+1)^{h}$ combinations of values $u_{1}, \ldots, u_{h}$, we obtain a linear system of $(m+1)^{h}$ unknowns $a_{k_{1}, \ldots, k_{h}}$, whose matrix is the Kronecker product $A \stackrel{\text { def }}{=} A_{1} \otimes A_{2} \otimes \cdots \otimes A_{h}$. Since $\operatorname{det}\left(A_{i}\right) \neq 0$ for all $i$, it follows that $\operatorname{det}(A) \neq 0$. This implies that the system has a single solution, $a_{k_{1}, \ldots, k_{h}}=0$ for all $k_{1}, \ldots, k_{h}$, proving that the polynomials are linearly independent.

LEMMA 3.9. Let $f\left(z_{1}, \ldots, z_{h}\right)$ be a polynomial of degree $\leq m$ in every $z_{i}$, such that $f \neq \equiv$. For all $i=1, h$, let $A_{i} \subseteq \mathbb{R}$ be a set of $m+1$ distinct, real values. Then there exists values $u_{i} \in A_{i}$, for $i=1, h$, such that $f\left(u_{1}, \ldots, u_{h}\right) \neq 0$.

Proof. By induction on $h$. When $h=1$, then $f$ has at most $m$ roots, hence there is at least one value $u_{1} \in A_{1}$ such that $f(u) \neq 0$. When $h>1$, consider any value $a \in A_{h}$, and set $z_{h}=a$. If $f\left[z_{h}:=\right.$ a] $\not \equiv 0$, then by induction on $h$ we get values $u_{1} \in A_{1}, \ldots, u_{h-1} \in$ $A_{h-1}$ for the other $h-1$ variables such that $f\left[z_{1}:=u_{1}, \ldots, z_{h-1}:=\right.$ $\left.u_{h-1}, z_{h}:=a\right] \neq 0$. If $f\left[z_{h}:=a\right] \equiv 0$ for all $m+1$ values $a \in A_{h}$, then $f$ can be divided by the polynomial $\prod_{a \in A_{h}}\left(z_{h}-a\right)$, which has degree $m+1$, contradiction.

Lemma 3.10. Let $_{g_{1}}, g_{2}, \ldots, g_{N}$ be linearly independent multivariate polynomials, in $h$ variables $y_{1}, \ldots, y_{h}$. Let $H: A\left(\subseteq \mathbb{R}^{h}\right) \rightarrow \mathbb{R}^{h}$ be a differentiable function such that its facobian $\mathcal{J}(H) \neq 0$ at some interior point in $A$, and define $f_{i}\left(z_{1}, \ldots, z_{h}\right) \stackrel{\text { def }}{=} g_{i}\left(H\left(z_{1}, \ldots, z_{h}\right)\right)$ for every $i$. Then the functions $f_{1}, \ldots, f_{N}$ are linearly independent.

Proof. Assume, by contradiction, that there exist constants $a_{1}, \ldots, a_{N}$ not all of which are 0 such that: $F=a_{1} f_{1}+a_{2} f_{2}+\cdots+$ $a_{N} f_{N} \equiv 0$. We prove that $G=a_{1} g_{1}+a_{2} g_{2}+\cdots+a_{N} g_{N} \equiv 0$, which is a contradiction. Let $\mathbf{v}=\left(v_{1}, \ldots, v_{h}\right) \in A$ be a point where $\mathcal{J}(H) \neq 0$. By the inverse function theorem, the function $H$ is invertible in some neighborhood of $H(\mathbf{v})$. Assume w.l.o.g. that this neighborhood is a product of open intervals, $\prod_{i}\left(\alpha_{i}, \beta_{i}\right)$, where $\alpha_{i}<\beta_{i}$, and let $A_{i} \subseteq\left(\alpha_{i}, \beta_{i}\right)$ be any finite set with $m+1$ distinct values, for each $i=1, h$. Since $H$ is invertible on $A_{1} \times \cdots \times A_{h}$, for any combination of values $\mathbf{u} \in A_{1} \times \cdots \times A_{h}$ there exists $\mathbf{w} \in \mathbb{R}^{h}$ such that $H(\mathbf{w})=\mathbf{u}$. By assumption, $F(\mathbf{w})=0$, and this implies $G(\mathbf{u})=G(H(\mathbf{w}))=F(\mathbf{w})=0$. Lemma 3.9 implies that $G \equiv 0$, which is a contradiction.

Lemma 3.11. Let $c_{1}, \ldots, c_{h}$ be distinct real values, and let $H$ : $\mathbb{R}^{h} \rightarrow \mathbb{R}^{h}$ be the following function: $H(\mathbf{z})=\mathbf{y}$, where:

$$
y_{i} \stackrel{\text { def }}{=} \prod_{j=1, h}\left(c_{i}+z_{j}\right)
$$

Let $\mathbf{u}=\left(u_{1}, \ldots, u_{h}\right)$ be any point with distinct coordinates, i.e. $u_{i} \neq$ $u_{j}$, such that $u_{i}+c_{j} \neq 0$ for all $i, j$. Then the facobian of $H$ at $\mathbf{u}$ is nonzero, $\mathcal{J}(H)(\mathbf{u}) \neq 0$.

Proof. Recall the definition of the Jacobian:

$$
\mathcal{J}(H) \stackrel{\text { def }}{=}\left[\begin{array}{ccc}
\frac{\partial y_{1}}{\partial z_{1}} & \cdots & \frac{\partial y_{1}}{\partial z_{h}} \\
\ddots & \ddots & \ddots \\
\frac{\partial y_{h}}{\partial z_{1}} & \cdots & \frac{\partial y_{h}}{\partial z_{h}}
\end{array}\right]
$$

Since $y_{i}=\prod_{j=1}^{h}\left(c_{i}+z_{j}\right)$ then $\frac{\partial y_{i}}{\partial z_{k}}=\prod_{j \neq k}\left(c_{i}+z_{j}\right)$. For each row $i \in[1, h]$, we factor out the product $\prod_{j=1}^{h}\left(c_{i}+z_{j}\right)$. This results in the following matrix: $\mathcal{J}^{\prime} \stackrel{\text { def }}{=}\left[\begin{array}{ccc}\frac{1}{c_{1}+z_{1}} & \cdots & \frac{1}{c_{1}+z_{h}} \\ \ddots & \ddots & \ddots \\ \frac{1}{c_{h}+z_{1}} & \cdots & \frac{1}{c_{h}+z_{h}}\end{array}\right]$. We note that $\operatorname{det}(\mathcal{J}(H))=\operatorname{det}\left(\mathcal{J}^{\prime}\right) \cdot \prod_{i=1}^{h} \prod_{j=1}^{h}\left(c_{i}+z_{j}\right)$. Therefore, if $\operatorname{det}\left(\mathcal{J}^{\prime}\right) \neq 0$ then $\operatorname{det}(\mathcal{J}) \neq 0$. The expression for $\operatorname{det}\left(\mathcal{J}^{\prime}\right)$ has a closed form [8]:

$$
\operatorname{det}\left(\mathcal{J}^{\prime}\right)=\frac{\prod_{1 \leq i<j \leq h}\left(c_{i}-c_{j}\right)\left(z_{i}-z_{j}\right)}{\prod_{1 \leq i<j \leq h}\left(c_{i}+z_{j}\right)}
$$

When $z_{j}=u_{j}$ for all $j$, then this value is $\neq 0$ because the $c_{i}$ 's are distinct, and the $u_{i}$ 's are distinct. 
For the next two statements we fix $c_{1}, \ldots, c_{h}$ to distinct real values and, for each $k_{1}, \ldots, k_{h} \in\{0, \ldots, m\}$, we define the following polynomial in variables $\mathbf{z}=\left(z_{1}, \ldots, z_{h}\right)$ :

$$
f_{k_{1}, \ldots, k_{h}}(\mathbf{z}) \stackrel{\text { def }}{=} \prod_{i=1}^{h} \prod_{j=1}^{h}\left(c_{i}+z_{j}\right)^{k_{i}}
$$

COROLLARy 3.12. The polynomials $f_{k_{1}, \ldots, k_{h}}$ of (22) are linearly independent.

Proof. By Lemma 3.8, the polynomials $g_{k_{1}, \ldots, k_{h}}(\mathrm{y}) \stackrel{\text { def }}{=} \prod_{i=1}^{h} y_{i}^{k_{i}}$ are linearly independent. By Lemma 3.11, the transformation $\mathbf{z} \mapsto \mathbf{y}$ given by (20) has a non-zero Jacobian (at some point). Then, by Lemma 3.10 it follows that the polynomials $f_{k_{1}, \ldots, k_{h}}(\mathbf{z})$ in (22) are also linearly independent.

Lemma 3.13. For each $i=1, h$, let $A_{i} \subseteq \mathbb{R}$ be a set of $m+1$ distinct, real values. Consider the following $(m+1)^{h} \times(m+1)^{h}$ matrix, whose rows are indexed by $\mathbf{u} \in A_{1}, \ldots, A_{h}$, and whose columns are indexed by $\mathbf{k} \in\{0,1,2, \cdots, m\}^{h}:$

$$
M_{\mathbf{u}, \mathbf{k}} \stackrel{\operatorname{def}}{=} \prod_{i=1}^{h} \prod_{j=1}^{h}\left(c_{i}+u_{j}\right)^{k_{i}}
$$

Then $M$ is non-singular.

Proof. We notice that each row of the matrix $M$ consists of the polynomials $f_{k_{1}, \ldots, k_{h}}(\mathbf{z})$ applied to some point in $\mathbf{u}$. We construct a matrix $M^{\prime}$ such that $\operatorname{det}\left(M^{\prime}\right) \neq 0$ and $M^{\prime} \operatorname{differs}$ from $M$ only by permutations of rows and columns. We construct, by induction on $k$, a $k \times(m+1)^{h}$ matrix $M_{k}$ such that each row consists of the values of the polynomials $f_{k_{1}, \ldots, k_{h}}(\mathbf{z})$ applied to some point in $\mathbf{u} \in A_{1} \times \cdots \times A_{h}$, and such that the $k \times k$ minor consisting of the first $k$ columns in $M_{k}$ is non-singular. When $k=1$ we choose any $\mathbf{u} \in A_{1} \times \cdots \times A_{h}$, and the statement holds because $M_{\mathbf{u},(0,0, \ldots, 0)}=1$ (i.e. all entries of the column $k_{1}=\cdots=k_{h}=0$ are 1 ). We show now how to construct $M_{k+1}$ by extending $M_{k}$. First, extend $M_{k}$ with a row consisting of the polynomials $f_{k_{1}, \ldots, k_{h}}(\mathbf{z})$. Let $D$ be the $(k+1) \times(k+1)$ minor $M_{k+1}$ defined by the first $k+1$ columns. $D$ is a linear combination of these polynomials, where the coefficient of each polynomial is the $k \times k$ minor consisting of the first $k+1$ columns except that containing the polynomial. By induction, the coefficient given by the minor consisting of the first $k$ columns is non-zero. By Corollary 3.12, $D(\mathbf{z})$ (viewed as a polynomial in $\mathbf{z}$ ) is non-zero, hence by Lemma 3.9 there exists $\mathbf{u} \in A_{1} \times \cdots \times A_{h}$ such that $D(\mathbf{u}) \neq 0$, proving the claim. Thus, we obtain $M^{\prime} \stackrel{\text { def }}{=} M_{(m+1)^{h}}$, a matrix that is non-singular. Since the matrix is non-singular, no two rows in $M^{\prime}$ can use the same value $\mathbf{u} \in A_{1} \times \cdots \times A_{h}$, and since both the number of rows in $M^{\prime}$ and the cardinality of the set $A_{1} \times \cdots \times A_{h}$ are the same, $(m+1)^{h}$, it follows that $M^{\prime}$ contains precisely the same rows as $M$, up to a permutation, proving the lemma.
Finally, we can now prove Theorem 3.7. For that we use the fact that $k_{0}=m-\left(k_{1}+\cdots+k_{h}\right)$ and write:

$$
M_{\mathrm{p}, \mathbf{k}}=y_{0}^{m}(\mathbf{p}) \prod_{i=1, h}\left(\frac{y_{i}(\mathbf{p})}{y_{0}(\mathbf{p})}\right)^{k_{i}}
$$

Since every row $\mathbf{p}$ in $M$ has the same factor $y_{0}^{m}(\mathbf{p})$, it suffices to prove that the matrix $M^{\prime}$ without this factor is non-singular:

$$
\begin{aligned}
& M_{\mathrm{p}, \mathbf{k}}^{\prime}=\prod_{i=1, h}\left(\frac{y_{i}(\mathbf{p})}{y_{0}(\mathbf{p})}\right)^{k_{i}}=\prod_{i=1, h} \prod_{j=1, h}\left(\frac{a_{i} \lambda_{1}^{p_{j}}+b_{i} \lambda_{2}^{p_{j}}}{a_{0} \lambda_{1}^{p_{j}}+b_{0} \lambda_{2}^{p_{j}}}\right)^{k_{i}} \\
&=\prod_{i=1, h} \prod_{j=1, h}\left(\frac{a_{i}+b_{i}\left(\frac{\lambda_{2}}{\lambda_{1}}\right)^{p_{j}}}{a_{0}+b_{0}\left(\frac{\lambda_{2}}{\lambda_{1}}\right)^{p_{j}}}\right)^{k_{i}} \\
&=\prod_{i=1, h} \prod_{j=1, h}\left(\frac{b_{i}}{b_{0}}+\frac{a_{i}-\frac{a_{0} b_{i}}{b_{0}}}{a_{0}+b_{0}\left(\frac{\lambda_{2}}{\lambda_{1}}\right)^{p_{j}}}\right)^{k_{j}} \\
& \stackrel{\text { def }}{=} \prod_{i=1, h} \prod_{j=1, h}\left(c_{i}+\frac{d_{i}}{a_{0}+b_{0}\left(\frac{\lambda_{2}}{\lambda_{1}}\right)^{p_{j}}}\right)^{k_{j}}
\end{aligned}
$$

We now use Lemma 3.13. Since $\lambda_{2} / \lambda_{1} \notin\{-1,0,+1\}$, the function $p_{j} \mapsto z_{j} \stackrel{\text { def }}{=} \frac{d_{i}}{a_{0}+b_{0}\left(\frac{\lambda_{2}}{\lambda_{1}}\right)^{p_{j}}}$ is injective, therefore the $m+1$ distinct values $p_{j}=1,2,3, \ldots, m+1$ will yield $m+1$ distinct values of $z_{j}$. By Lemma 3.13, the matrix $M^{\prime}$ is non-singular, completing the proof of Theorem 3.7.

\subsection{Designing the blocks $B(u, v)$}

In this section, we design the block $\operatorname{TID} B(u, v)$ such that the probability of the lineage of $Q$ over $B(u, v)$ can be expressed as in (19), and that it meets the conditions of (16)-(18).

We design our blocks to prove hardness for final type-I queries, where every endpoint (i.e., $u$ and $v$ ) is assigned one of two values $\{0,1\}$. Since we design symmetric blocks, then $h=\{00,10,11\}$. In our construction, each block is parameterized by a pair $\mathbf{p}=\left\{p_{1}, p_{2}\right\}$. The main focus of this section is a block that is parameterized by a single value $p$, denoted $B_{p}(u, v)$. We show how two blocks $B_{p_{1}}(u, v), B_{p_{2}}(u, v)$ can be combined in parallel to create a block $B_{\mathbf{p}}(u, v)$ that has the desired form and properties.

The Block $B_{p}(u, v)$. The bipartite domain of $B_{p}(u, v)$ is $V_{1}(p) \cup V_{2}(p)$, and is defined as follows:

$$
\begin{aligned}
& V_{1}(p)=\{u, v\} \cup\left\{r_{k}: k \in[1, p-1]\right\} \\
& V_{2}(p)=\left\{t_{k}: k \in[1, p]\right\}
\end{aligned}
$$

where the $r_{k}$ and $t_{k}$ are fresh constants. The tuple probabilities of $B_{p}(u, v)$ are:

$$
\operatorname{Pr}(R(u))=\left\{\begin{array}{cc}
c & u \in V_{1}(p) \\
1 & \text { otherwise }
\end{array} \quad \operatorname{Pr}(T(v))= \begin{cases}c & v \in V_{2}(p) \\
1 & \text { otherwise }\end{cases}\right.
$$

where $c \in(0,1)$ is a constant. For every binary symbol $S \in \mathcal{R}$ the probabilities are:

- $\operatorname{Pr}\left(S\left(u, t_{1}\right)\right)=c$. 
- $\operatorname{Pr}\left(S\left(v, t_{p}\right)\right)=c$.

- $\operatorname{Pr}\left(S\left(r_{k}, t_{k}\right)\right)=c$ for all $k \in[1, p-1]$.

- $\operatorname{Pr}\left(S\left(r_{k}, t_{k+1}\right)\right)=c$ for all $k \in[1, p-1]$.

- Otherwise, $\operatorname{Pr}(S(a, b))=1$.

Example 3.14. The graphical representation of $B_{p}(u, v)$ is:

$$
u \stackrel{\text { def }}{=} r_{0}-t_{1}-r_{1}-\cdots-r_{p-1}-t_{p}-r_{p} \stackrel{\text { def }}{=} v
$$

The binary atoms corresponding to the edges of the path have a probability of $c$, and atoms corresponding to non-edges (e.g., $\left.S\left(t_{1}, t_{3}\right)\right)$ have a probability of 1 . Also note that $r_{0} \stackrel{\text { def }}{=} u$, and $r_{p} \stackrel{\text { def }}{=} v$.

Let $p \geq 1$ be a natural number. The lineage of $Q$ over the block TID $B_{p}(u, v)$ is denoted $Y^{(p)}(u, v)$, and its arithmetization $y(p)$. That is, $y(p)$ is the multilinear polynomial representing the probability $\operatorname{Pr}\left(Y^{(p)}(u, v)\right)$. We associate the endpoints $u$ and $v$ with the unary atoms $R(u)$ and $R(v)$ respectively. Thus $Y_{a b}^{(p)}(u, v) \stackrel{\text { def }}{=}$ $Y^{(p)}(u, v)[R(u):-a, R(v):-b]$, and $y_{a b}(p) \stackrel{\text { def }}{=} y(p)[R(u):-a, R(v):-b]$ is defined accordingly. The following proposition follows directly from the way we construct the blocks.

Proposition 3.15. Let $Y^{(p)}(u, v)$ denote the lineage of $Q$ over a p-length block-TID $B_{p}(u, v)$. Then $Y^{(p)}(u, v)=Y^{(p)}(v, u)$.

Since $Y^{(p)}(u, v)=Y^{(p)}(v, u)$, then $Y_{a b}^{(p)}(u, v)=Y_{a b}(v, u)=$ $Y_{b a}(u, v)$. Accordingly, we have that $y_{a b}(p)=y_{b a}(p)$.

We define $z_{a b}(p)$ to be the valuation of $y_{a b}(p)$ when all of the RVs in $Y_{a b}^{(p)}(u, v)$ have a probability of $c$. For any natural $p \geq 1$ :

$$
z_{a b}(p) \stackrel{\text { def }}{=} y_{a b}(p)[c, \ldots, c]
$$

In the rest of this section we design the block $B_{p}(u, v)$ and prove that it meets the following conditions.

Theorem 3.16. Let $B_{p}(u, v)$ be a block TID, parameterized by a parameter $p \geq 1$. For every $i \in\{00,10,11\}$ it holds that:

$$
z_{i}(p)=\left(a_{i} \lambda_{1}^{p}+b_{i} \lambda_{2}^{p}\right) \quad \forall i \in\{00,10,11\}
$$

where $\lambda_{1}, \lambda_{2}$ and $a_{i}, b_{i}$ are constants, independent of $p$, satisfying:

$$
\begin{array}{rrr}
\lambda_{1} \neq \pm \lambda_{2} & \text { and } & \lambda_{1} \neq 0, \lambda_{2} \neq 0 \\
b_{i} \neq 0 & \forall i \in\{00,10,11\} \\
a_{i} b_{j} \neq a_{j} b_{i} & i \neq j
\end{array}
$$

Let $\mathbf{p}=\left\{p_{1}, p_{2}\right\}$. We now describe how to create the block $B_{\mathbf{p}}(u, v)$ that has probability $y_{i}(\mathbf{p})($ as in (19)) for every $i \in\{00,10,11\}$ To do so, we construct two disjoint blocks between nodes $u$ and $v$ with parameters $p_{1}, p_{2}$ respectively (see Figure 1 ). The lineage of this block is $Y_{i}^{\left(p_{1}\right)}(u, v) \wedge Y_{i}^{\left(p_{2}\right)}(u, v)$. Since the blocks are disjoint, then $\operatorname{var}\left(Y^{\left(p_{1}\right)}(u, v)\right) \cap \operatorname{var}\left(Y^{\left(p_{2}\right)}(u, v)\right)=\{R(u), R(v)\}$. In particular, the Boolean functions $Y_{i}^{\left(p_{1}\right)}(u, v)$ and $Y_{i}^{\left(p_{2}\right)}(u, v)$ are disjoint for every $i \in\{00,10,11\}$. So, we get that:

$y_{a b}(\mathbf{p})=\operatorname{Pr}\left(\bigwedge_{j=1}^{2} Y_{a b}^{\left(p_{j}\right)}(u, v)\right)=\prod_{j=1}^{2} \operatorname{Pr}\left(Y_{a b}^{\left(p_{j}\right)}(u, v)\right)=y_{a b}\left(p_{1}\right) y_{a b}\left(p_{2}\right)$

Consequently, once we prove Theorem 3.16, the conditions of (16)(18) follow from (30).

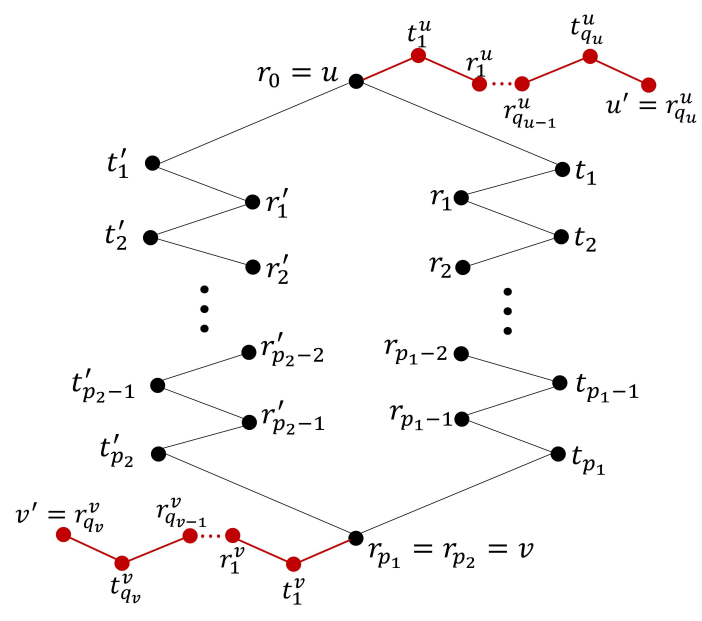

Figure 1: Block $B_{\mathbf{p}}(u, v)$ where $\mathbf{p}=\left\{p_{1}, p_{2}\right\}$, and blocks $B_{q_{u}}\left(u, u^{\prime}\right)$ and $B_{q_{v}}\left(v, v^{\prime}\right)$.

To prove Theorem 3.16 we first show that $Y^{(p)}(u, v)$ is a connected Boolean function. Consequently, we can apply Lemma 1.2 to argue that the determinant of the "small matrix" associated with $y(p)$ (see (1)) is not identically zero, and, by Lemma 1.1, there are probability values in $\{0, c, 1\}$ for which the small matrix is nonsingular. In fact, we prove something stronger: if $Q$ is final, then the matrix remains nonsingular even if we set all probabilities to $c$. Some further analysis of this matrix, along with two simple properties of the lineage $Y^{(p)}(u, v)$ allow us to prove Theorem 3.16.

We recall that $Q(s, t)$ is the Boolean formula that results from mapping $x \mapsto s$ and $y \mapsto t$. We note that $Q(s, t)$ is identically true whenever $(s, t) \notin\left\{\left(u, t_{1}\right),\left(v, t_{p}\right),\left(r_{k}, t_{k}\right),\left(r_{k}, t_{k+1}\right) \mid k \in[1, p-1]\right\}$. Therefore:

$$
Y^{(p)}(u, v)=\bigwedge_{i=1}^{p} Q\left(r_{i-1}, t_{i}\right) \wedge Q\left(r_{i}, t_{i}\right)
$$

where $r_{0}=u$ and $r_{p}=v$. In particular, when $p=1$ then $Y^{(1)}(u, v)=$ $Q\left(u, t_{1}\right) \wedge Q\left(v, t_{1}\right)$.

We say that a Boolean function $f$ is disconnected if $f=f_{1} \wedge f_{2}$ where $f_{1}$ and $f_{2}$ are non-constant, disjoint Boolean functions (i.e., $\left.\operatorname{var}\left(f_{1}\right) \cap \operatorname{var}\left(f_{2}\right)=\emptyset\right)$. Otherwise, we say that $f$ is connected.

Lemma 3.17. If $Q$ is an unsafe type-I query, then $Y^{(p)}(u, v)$ is connected.

Proof. By induction on $p$. Since $Q$ is unsafe, it is connected. Therefore, it must hold that $h_{1} \stackrel{\text { def }}{=} Q\left(u, t_{1}\right)$ and $h_{2} \stackrel{\text { def }}{=} Q\left(v, t_{1}\right)$ are connected. Observe that when $p=1$ then $Y^{(1)}(u, v)=h_{1} \wedge h_{2}$. Since $T\left(t_{1}\right) \in \operatorname{var}\left(h_{1}\right) \cap \operatorname{var}\left(h_{2}\right)$ then $h_{1}$ and $h_{2}$ are not disjoint. Therefore, $Y^{(1)}(u, v)$ is disconnected only if there is some homomorphism $C\left(u, t_{1}\right) \rightarrow C_{R}\left(u, t_{1}\right)\left(\right.$ or $\left.C\left(v, t_{1}\right) \rightarrow C_{R}\left(v, t_{1}\right)\right)$ where $C$ is a clause in $Q_{\text {left }} \wedge Q_{\text {middle }}$ and $C_{R}$ is a right clause. We note that $C \notin Q_{\text {left }}$ because all left clauses, and only left clauses, contain the unary symbol $R$. If $C \in Q_{\text {middle }}$, it means that $C_{R}$ is a redundant clause, which is a contradiction (we assume that all clauses of $Q$ are non-redundant). Symmetrically, for any $k \in[1, p-1]$ it holds 
that $Q\left(r_{k}, t_{k}\right) \wedge Q\left(r_{k}, t_{k+1}\right)$ are connected via the unary atom $R\left(r_{k}\right)$, and thus connected.

Let $p>1$. Observe that $Y^{(p)}(u, v)=Y^{(p-1)}\left(u, r_{p-1}\right) \wedge Y^{(1)}\left(r_{p-1}, v\right)$ where $Y^{(1)}\left(r_{p-1}, v\right)=Q\left(r_{p-1}, t_{p}\right) \wedge Q\left(v, t_{p}\right)$. By the induction hypothesis, $Y^{(p-1)}\left(u, r_{p-1}\right)$ and $Y^{(1)}\left(r_{p-1}, v\right)$ are both connected. In addition, $Y^{(p-1)}\left(u, r_{p-1}\right)$ and $Y^{(1)}\left(r_{p-1}, v\right)$ are connected via the unary left atom $R\left(r_{p-1}\right)$ that appears in both Boolean formulas. By the previous reasoning none of the clauses containing $R\left(r_{p-1}\right)$ become redundant and hence, $Y^{p}(u, v)$ is connected.

Note, in particular, that Lemma 3.17 holds for final queries.

Let $N$ be the number of RVs in $y_{a b}(1)$. We define the matrix of polynomials:

$$
A \stackrel{(1)}{\stackrel{\text { def }}{=}}\left[\begin{array}{ll}
y_{00}(1) & y_{01}(1) \\
y_{10}(1) & y_{11}(1)
\end{array}\right]
$$

and the polynomial $f_{A}:[0,1]^{N} \rightarrow[0,1]$ associated with the determinant of $A^{(1)}$ :

$$
f_{A}\left(u_{1}, \ldots, u_{N}\right) \stackrel{\text { def }}{=} \operatorname{det}\left(A^{(1)}\right)=y_{00}(1) y_{11}(1)-y_{01}(1) y_{10}(1)
$$

and observe that $f_{A}$ is a degree- 2 polynomial. The previous lemma, and Lemma 1.2 from the introduction, imply that $f_{A} \not \equiv 0$. Therefore, by Lemma 1.1, there exists an assignment $\boldsymbol{u}$ of the variables of $f_{A}$ with probabilities in $\{0, c, 1\}$ such that $f_{A}(\boldsymbol{u}) \neq 0$. Next, we show a stronger result: if $Q$ is a final query, then $f_{A}(\boldsymbol{u}) \neq 0$ for any assignment $\boldsymbol{u}:\left\{u_{1}, \ldots, u_{N}\right\} \rightarrow(0,1)^{N}$, and in particular, $f_{A}(c, \ldots, c) \neq 0$.

Theorem 3.18. If $u_{i} \in(0,1)$ for all $i \in[1, N]$ then $f_{A}\left(u_{1}, \ldots, u_{N}\right) \neq 0$.

To prove Theorem 3.18, we use the fact that $Q$ is final, and prove:

Lemma 3.19. Let $Q$ be a final Type-I query, and let $X$ be a Boolean $R V$ in $Y^{(p)}(u, v)$ corresponding to any atom other than $R(u)$ and $R(v)$. Then $Y^{(p)}[X:-a]$ is disconnected for $a \in\{0,1\}$.

Proof. For $i \geq 1$, we define: $f(i) \stackrel{\text { def }}{=} Q\left(r_{i-1}, t_{i}\right) \wedge Q\left(r_{i}, t_{i}\right)$.

Case 1: $X=R\left(r_{k}\right)$ for some $k \in[1, p-1]$ (we let $r_{0}=u$, and $\left.r_{p}=v\right)$. We note that $Y^{(p)}=h_{1} \wedge h_{2}$ where $h_{1} \stackrel{\text { def }}{=} \bigwedge_{i=1}^{k} f(i)$ and $h_{2} \stackrel{\text { def }}{=} \bigwedge_{i=k+1}^{p} f(i)$. Since $\operatorname{var}\left(h_{1}\right) \cap \operatorname{var}\left(h_{2}\right)=\{X\}$, the result follows.

Case 2: $X=T\left(t_{k}\right)$ for some $k \in[1, p]$. We express $Y^{(p)}=h_{1} \wedge h_{2}$ where $h_{1} \stackrel{\text { def }}{=} \bigwedge_{i=1}^{k-1} f(i) \wedge Q\left(r_{k-1}, t_{k}\right)$ and $h_{2} \stackrel{\text { def }}{=} Q\left(r_{k}, t_{k}\right) \bigwedge_{i=k+1}^{p} f(i)$. The result follows from noting that $\operatorname{var}\left(h_{1}\right) \cap \operatorname{var}\left(h_{2}\right)=\{X\}$.

Case 3: $X=S\left(r_{k}, t_{k}\right)$ for some binary symbol $S \in \operatorname{Symb}(Q)$ where $k \in[1, p]$. Consider the Boolean function $Q\left(r_{k}, t_{k}\right)$. Since $Q$ is final, then setting a value to $S\left(r_{k}, t_{k}\right)$ makes it safe. Hence, it does one of the following: (1) Makes $Q\left(r_{k}, t_{k}\right)$ disconnected, (2) Makes $Q_{\text {left }}\left(r_{k}, t_{k}\right)$ redundant or, (3) Makes $Q_{\text {right }}\left(r_{k}, t_{k}\right)$ redundant. If (1) then we are done because if $Q\left(r_{k}, t_{k}\right)\left[S\left(r_{k}, t_{k}\right)\right]$ is disconnected, then clearly so is $Y^{(p)}$. Otherwise, since (2) is equivalent to setting $R\left(r_{k}\right) \leftarrow 1$ and since (3) is equivalent to setting $T\left(t_{k}\right) \leftarrow 1$ then the result follows from cases 1 and 2 respectively.

Corollary 3.20. There exists some constant $\alpha \neq 0$ such that: $f_{A}=\alpha \prod_{i=1}^{N} u_{i}\left(1-u_{i}\right)$

Proof. From Lemma 1.2 it follows that $f_{A}\left[u_{i}:=0\right]=f_{A}\left[u_{i}:=\right.$ $1]=0$. Therefore, it follows that $f_{A}$ is divisible by $u_{i}\left(1-u_{i}\right)$, for every $i$. Hence $f_{A}=\alpha \prod_{i=1}^{N} u_{i}\left(1-u_{i}\right)$ and, since each variable has degree $\leq 2$ in $f_{A}$, it follows that $\alpha$ is a constant.
Theorem 3.18 follows from Corollary 3.20 because $f_{A}(\boldsymbol{u}) \neq 0$ for any assignment $\boldsymbol{u}: \operatorname{var}\left(f_{A}\right) \rightarrow(0,1)^{N}$. Thus, we have established that $f_{A}(c, \ldots, c) \neq 0$. That is, $\operatorname{det}\left(A^{(1)}\right) \neq 0$ when the real variables in $y(1)$ have a uniform value of $c$. In other words, we have shown that the matrix:

$$
A^{(1)} \stackrel{\text { def }}{=}\left[\begin{array}{ll}
z_{00}(1) & z_{01}(1) \\
z_{10}(1) & z_{11}(1)
\end{array}\right]
$$

is invertible.

So far, we have discussed only the "small" matrix $A^{(1)}$ that corresponds to one step of the zig-zag block $B_{p}(u, v)$; in other words, this is the matrix that we have for $B_{1}(u, v)$, when $p=1$. Next, we show how to compute $A^{(p)}$. We define:

$$
A^{(p)} \stackrel{\text { def }}{=}\left[\begin{array}{ll}
z_{00}(p) & z_{01}(p) \\
z_{10}(p) & z_{11}(p)
\end{array}\right]
$$

Lemma 3.21. When $p \geq 1$ then $A^{(p)}=\left[A^{(1)} \cdot C\right]^{p-1} \cdot A^{(1)}$ where $C=\left[\begin{array}{cc}1-c & 0 \\ 0 & c\end{array}\right]$. When $p=0$ then $A^{(p)}=\mathbb{I}$ where $\mathbb{I}$ is the identity matrix.

Proof. For the case where $p \geq 1$, the proof is by induction on $p$. The base case for $p=1$ is immediate. So, we assume correctness for $p-1$ and prove for $p$.

$$
\begin{aligned}
Y^{(p)}(u, v) & \stackrel{\text { def }}{=} \bigwedge_{(s, t) \in B_{p}(u, v)} Q(s, t) \\
& =\underbrace{\bigwedge_{(s, t) \in B_{p-1}\left(r_{0}, r_{p-1}\right)} Q(s, t)}_{Y^{(p-1)}\left(r_{0}, r_{p-1}\right)} \underbrace{\bigwedge_{(s, t) \in B_{1}\left(r_{p-1}, r_{p}\right)} Q(s, t)}_{Y^{(1)}\left(r_{p-1}, r_{p}\right)}
\end{aligned}
$$

We note that the only atom common to both $Y^{(p-1)}\left(r_{0}, r_{p-1}\right)$ and $Y^{(1)}\left(r_{p-1}, r_{p}\right)$ is $R\left(r_{p-1}\right)$. Since $\operatorname{Pr}\left(R\left(r_{p-1}\right)=1\right)=c$, we have that:

$$
z_{a b}(p)=(1-c)\left(z_{a 0}(p-1)\right)\left(z_{0 b}(1)\right)+c\left(z_{a 1}(p-1)\right)\left(z_{1 b}(1)\right)
$$

Writing (36) in matrix terms and applying the induction hypothesis, we get that:

$$
\begin{aligned}
A^{(p)} & =\left[\begin{array}{ll}
z_{00}(p) & z_{01}(p) \\
z_{10}(p) & z_{11}(p)
\end{array}\right] \\
& =\left[\begin{array}{ll}
z_{00}(p-1) & z_{01}(p-1) \\
z_{10}(p-1) & z_{11}(p-1)
\end{array}\right]\left[\begin{array}{cc}
1-c & 0 \\
0 & c
\end{array}\right]\left[\begin{array}{ll}
z_{00}(1) & z_{01}(1) \\
z_{10}(1) & z_{11}(1)
\end{array}\right] \\
& =\left[\left[\begin{array}{ll}
z_{00}(1) & z_{01}(1) \\
z_{10}(1) & z_{11}(1)
\end{array}\right]\left[\begin{array}{rr}
1-c & 0 \\
0 & c
\end{array}\right]\right]^{p-2}\left[\begin{array}{ll}
z_{00}(1) & z_{01}(1) \\
z_{10}(1) & z_{11}(1)
\end{array}\right] \\
& \cdot\left[\begin{array}{rr}
1-c & 0 \\
0 & c
\end{array}\right]\left[\begin{array}{ll}
z_{00}(1) & z_{01}(1) \\
z_{10}(1) & z_{11}(1)
\end{array}\right] \\
& =\left[\left[\begin{array}{ll}
z_{00}(1) & z_{01}(1) \\
z_{10}(1) & z_{11}(1)
\end{array}\right]\left[\begin{array}{rr}
1-c & 0 \\
0 & c
\end{array}\right]\right]^{p-1}\left[\begin{array}{ll}
z_{00}(1) & z_{01}(1) \\
z_{10}(1) & z_{11}(1)
\end{array}\right]
\end{aligned}
$$

where (37) follows from writing $z_{a b}(p)$ in matrix terms, (38) follows from the induction hypothesis, and (39) from simplification.

When $p=0$, we are basically looking at an empty lineage expression $Y^{(0)}(u, u)$, that is trivially true for $R(u) \in\{0,1\}$. In other words, $Y_{00}^{(0)}=Y_{11}^{(0)}=1$ and $Y_{01}^{(0)}=Y_{10}^{(0)}=0$.

An immediate corollary of Lemma 3.21 is that $A^{(p)}=\left[A^{(1)} C\right]^{p} C^{-1}$. It is easy to see that $C^{-1}=\left[\begin{array}{cc}\frac{1}{1-c} & 0 \\ 0 & \frac{1}{c}\end{array}\right]$. 
Let $\lambda_{1}$ and $\lambda_{2}$ be the eigenvalues of $A^{(1)}$ (defined in (34)). We prove now condition (27), which is the first of the three conditions that we need to establish. We require the following simple proposition.

Proposition 3.22. The following hold: (1) $z_{00}(1)<z_{01}(1)=$ $z_{10}(1)<z_{11}(1)$, and $(2) 0<z_{a b}(1) \leq 1$ for all $a, b \in\{0,1\}$

Proof. We note that $Y^{(1)}(u, v)$ and $Y^{(1)}(v, u)$ are identical. Consequently, $Y_{a b}^{(1)} \equiv Y_{b a}^{(1)}$ up to the renaming of the variables. Therefore, $z_{01}(1)=z_{10}(1)$. Since $Q$ does not contain negations, then its lineage is a monotonic Boolean function. Further, since the Boolean function $Y^{(1)}$ depends on both atoms $R(u)$ and $R(v)$, then (1) follows. Item (2) follows by noting that $Y_{00}^{(1)}$ is satisfiable for any final type-I query, thus $z_{00}(1)>0$.

In what follows, we define

$$
B \stackrel{\text { def }}{=}\left[A^{(1)} \cdot C\right]=\left[\begin{array}{l}
(1-c) z_{00}(1) c z_{01}(1) \\
(1-c) z_{10}(1) c z_{11}(1)
\end{array}\right]
$$

Lemma 3.23. Let $\lambda_{1}, \lambda_{2}$ be the eigenvalues of $B$ (see (40)). Then: $\lambda_{1} \neq 0, \lambda_{2} \neq 0$, and $\lambda_{1} \neq \pm \lambda_{2}$.

Proof. This follows immediately from $\lambda_{1}+\lambda_{2}=\operatorname{Tr}(B)=(1-$ c) $z_{00}+c z_{11}>0$ because $c \in(0,1)$ and by Proposition $3.220<$ $z_{00}<z_{11}$. Also, $\lambda_{1} \lambda_{2}=\operatorname{det}(B)=\operatorname{det}\left(A^{(1)}\right) \operatorname{det}(C) \neq 0$, and the fact that a matrix where all eigenvalues are equal is a diagonal matrix (which $B$ obviously is not).

For a more elementary argument, recall that the characteristic polynomial of $B$ (see (40)) is (we drop the parameter (1)):

$$
\operatorname{det}(\lambda I-B)=\lambda^{2}-\lambda\left(c z_{11}+(1-c) z_{00}\right)+c(1-c)\left(z_{00} z_{11}-z_{01} z_{10}\right)
$$

From (41) we see that $\lambda=0$ is a root of the characteristic polynomial iff $\operatorname{det}\left(A^{(1)}\right)=z_{00} z_{11}-z_{01} z_{10}=0$. By Theorem 3.18 , this cannot be the case. Therefore, $\lambda_{1}, \lambda_{2} \neq 0$. Also from (41) we get that the two roots of $\operatorname{det}\left(\lambda I-A^{(1)}\right)$ are:

$$
\lambda_{12}=\frac{\left(c z_{11}+(1-c) z_{00}\right) \pm \sqrt{\left(c z_{11}-(1-c) z_{00}\right)^{2}+4 c(1-c) z_{01} z_{10}}}{2} .
$$

Since $z_{01}=z_{10}>0$ (Proposition 3.22)) and since $c \in(0,1)$, it follows that $\lambda_{1} \neq \lambda_{2}$. Since, by Proposition $3.22,(1-c) z_{00}+c z_{11}>0$ it follows that $\lambda_{1} \neq \pm \lambda_{2}$.

Corollary 3.24. For every $p \geq 1$ it holds that $\operatorname{det}\left(A^{(p)}\right) \neq 0$. Or, that $y_{00}^{(p)} y_{11}^{(p)}-y_{01}^{(p)} y_{10}^{(p)} \neq 0$.

Proof. By Lemma 3.21, we have that $A^{(p)}=B^{p} C^{-1}$. Hence $\operatorname{det}\left(A^{(p)}\right)=0$ iff $\operatorname{det}(B)=0$. By Lemma 3.23, the two eigenvalues of $B$ are non-zero. Hence, $\operatorname{det}(B) \neq 0$.

The following Lemma is required to establish the fact that $\mathcal{N}$ is invertible (Proposition 3.5). We recall the definition of $y_{a}^{(t)}$ in (6).

Lemma 3.25. For every $a \in\{0,1\}$ and $t \geq 1$ it holds that $y_{a}^{(t)}>0$. Also, for every pair of integers $t_{2}>t_{1} \geq 1$ it holds that $\frac{y_{1}^{\left(t_{1}\right)}}{y_{0}^{\left(t_{1}\right)}} \neq \frac{y_{1}^{\left(t_{2}\right)}}{y_{0}^{\left(t_{2}\right)}}$.
Proof. The claim that $y_{a}^{(t)}>0$ follows by noting that $Y_{00}^{(1)}$ is satisfiable for any final type-I query and that $y_{a}^{(t)}=y_{a 0}^{(t)}+y_{a 1}^{(t)} \geq$ $y_{00}^{(t)}$ by Proposition 3.22 .

Now, we show that for any $d>0$ it holds that $y_{1}^{(t+d)} y_{0}^{(t)} \neq$ $y_{1}^{(t)} y_{0}^{(t+d)}$. We observe that for any $a \in\{0,1\}$ it holds that:

$$
\begin{aligned}
y_{a}^{(t+d)} & =y_{a 0}^{(t+d)}+y_{a 1}^{(t+d)} \\
& =y_{a 0}^{(t)} y_{00}^{(d)}+y_{a 1}^{(t)} y_{10}^{(d)}+y_{a 0}^{(t)} y_{01}^{(d)}+y_{a 1}^{(t)} y_{11}^{(d)}
\end{aligned}
$$

Hence, after simplifying the expression and using the fact that $y_{01}^{(t)}=y_{10}^{(t)}$ (Proposition 3.15), we get that

$$
y_{1}^{(t+d)} y_{0}^{(t)}-y_{1}^{(t)} y_{0}^{(t+d)}=\left(y_{11}^{(d)}-y_{00}^{(d)}\right)\left(y_{00}^{(t)} y_{11}^{(t)}-y_{01}^{(t)} y_{10}^{(t)}\right)
$$

By monotonicity and non-redundancy we get that for any $d \geq 1$ it holds that $\left(y_{11}^{(d)}-y_{00}^{(d)}\right)>0$. By Corollary 3.24 we have that $\left(y_{00}^{(t)} y_{11}^{(t)}-y_{01}^{(t)} y_{10}^{(t)}\right) \neq 0$ for all $t \geq 1$. This completes the proof. $\square$

Since $B$ has two distinct, non-zero eigenvalues, then it has two linearly independent eigenvectors. In other words, $B$ is diagonizable, and thus $B^{p}=P D^{k} P^{-1}$ where $P$ is the matrix whose columns are the eigenvectors of $B$, and $D$ is the diagonal matrix of its eigenvalues. Let $v_{1}=\left(c_{11}, c_{21}\right)^{T}$, and $v_{2}=\left(c_{12}, c_{22}\right)^{T}$ be the two linearly independent eigenvectors corresponding to eigenvalues $\lambda_{1}$ and $\lambda_{2}$ respectively. By Lemma 3.21, we have that $A^{(p)}=B^{p} \cdot C^{-1}$, and hence has the following form.

$$
\begin{aligned}
A^{(p)} & =B^{p} C^{-1}=\left(P D^{p} P^{-1}\right) C^{-1}=(P)\left(D^{p}\right)\left(P^{-1} C^{-1}\right) \\
& =\left[\begin{array}{ll}
c_{11} & c_{12} \\
c_{21} & c_{22}
\end{array}\right]\left[\begin{array}{cc}
\lambda_{1}^{p} & 0 \\
0 & \lambda_{2}^{p}
\end{array}\right]\left[\begin{array}{ll}
b_{11} & b_{12} \\
b_{21} & b_{22}
\end{array}\right] \\
& =\left[\begin{array}{ll}
c_{11} b_{11} \lambda_{1}^{p}+c_{12} b_{21} \lambda_{2}^{p} & c_{11} b_{12} \lambda_{1}^{p}+c_{12} b_{22} \lambda_{2}^{p} \\
c_{21} b_{11} \lambda_{1}^{p}+c_{22} b_{21} \lambda_{2}^{p} & c_{21} b_{12} \lambda_{1}^{p}+c_{22} b_{22} \lambda_{2}^{p}
\end{array}\right] \\
& =\left[\begin{array}{ll}
a_{00} \lambda_{1}^{p}+b_{00} \lambda_{2}^{p} & a_{01} \lambda_{1}^{p}+b_{01} \lambda_{2}^{p} \\
a_{10} \lambda_{1}^{p}+b_{10} \lambda_{2}^{p} & a_{11} \lambda_{1}^{p}+b_{11} \lambda_{2}^{p}
\end{array}\right] \\
& =\left[\begin{array}{ll}
a_{00} \lambda_{1}^{p}+b_{00} \lambda_{2}^{p} & a_{10} \lambda_{1}^{p}+b_{10} \lambda_{2}^{p} \\
a_{10} \lambda_{1}^{p}+b_{10} \lambda_{2}^{p} & a_{11} \lambda_{1}^{p}+b_{11} \lambda_{2}^{p}
\end{array}\right]
\end{aligned}
$$

where the transition from (43) to (44) is by defining $a_{00} \stackrel{\text { def }}{=} c_{11} b_{11}$, $b_{00} \stackrel{\text { def }}{=} c_{12} b_{21}$ etc. The transition from (44) to (45) follows from the fact that, by construction, $A^{(p)}$ is symmetric (see Proposition 3.15). Notice that this establishes Eq.(26), which we need to prove as part of Theorem 3.16. In particular, we have that:

$$
A^{(1)}=\left[\begin{array}{lll}
a_{00} \lambda_{1}+b_{00} \lambda_{2} & a_{10} \lambda_{1}+b_{10} \lambda_{2} \\
a_{10} \lambda_{1}+b_{10} \lambda_{2} & a_{11} \lambda_{1}+b_{11} \lambda_{2}
\end{array}\right]
$$

Proof of Theorem 3.16. From (45) it follows that $z_{i}(p)$ can be written in the form of (26) for all $i \in\{00,10,11\}$. In Lemma 3.23, we have shown that $\lambda_{1}, \lambda_{2} \neq 0$, and that $\lambda_{1} \neq \pm \lambda_{2}$, thus proving condition (27).

By Lemma 3.21 we have that $A^{(0)}=\left(A^{(1)}\right)^{0}=\mathbb{I}$ where $\mathbb{I}$ is the identity matrix. Therefore, from (45), we get the following three equations:

$$
a_{00}+b_{00}=1 \quad a_{11}+b_{11}=1 \quad a_{10}+b_{10}=0
$$

We show that $b_{i} \neq 0$ for all $i \in\{00,10,11\}$. If $b_{10}=0$ then, since $a_{10}=-b_{10}$ it follows that $z_{10}(1)=0$, which is a contradiction 
(Proposition 3.22). Thus, $b_{10} \neq 0$. Assume that $b_{00}=0$. This means that $a_{00}=1$, and that for any $p \geq 1$, we have that $z_{00}(p)=\lambda_{1}^{p}$ (see (45)). Recall that $z_{00}(p)=(1-c) z_{00}(p-1) z_{00}(1)+c z_{01}(p-$ 1) $z_{10}(1)$. By proposition 3.22 we have that $z_{01}(p-1)>z_{00}(p-1)$, and that $z_{10}(1)>z_{00}(1)$. Therefore, $z_{00}(p)>z_{00}(p-1) z_{00}(1)=$ $\lambda_{1}^{p-1} \lambda_{1}=\lambda^{p}$, and we arrive at a contradiction. Similarly, if $b_{11}=0$ then $a_{11}=1$, and $z_{11}(p)=\lambda_{1}^{p}$. Now, since $z_{11}(p)=(1-c) z_{10}(p-$ 1) $z_{01}(1)+c z_{11}(p-1) z_{11}(1)$, then since by Proposition $3.22 z_{11}(p)>$ $z_{10}(p)=z_{01}(p)$ for all $p \geq 1$, we have that $z_{11}(p)<\lambda_{1}^{p}$, which is a contradiction.

Finally, we show that $a_{i} b_{j} \neq a_{j} b_{i}$ for $i \neq j$. Assume, by contradiction, that $a_{00} b_{11}=a_{11} b_{00}$. Substituting $b_{11}=\left(1-a_{11}\right)$ and $b_{00}=\left(1-a_{00}\right)$, this implies that $a_{00}=a_{11}$ and thus $b_{00}=b_{11}$. But, by (46), this means that $z_{00}(1)=z_{11}(1)$ which, by Proposition 3.22 , is a contradiction. Now, assume, by contradiction, that $a_{00} b_{10}=a_{10} b_{00}$. From (47) we have that $-a_{10}=b_{10}$. Substituting, this gives us that $a_{00} b_{10}=-b_{10}\left(1-a_{00}\right)$ or, that $b_{10}=0$. But then $z_{10}(1)=0$, which, by Proposition 3.22 , is a contradiction. Symmetrically, it is shown that $a_{11} b_{10} \neq a_{10} b_{11}$. This completes the proof.

\section{CONCLUSIONS}

One can think of the model counting problem as: given a set of tuples to exclude, compute the number of models of a sentence that do not use any of the excluded tuples. In this paper we studied the generalized model counting problem, where we are also given a set of tuples to include, and need to count only those models that contain all these tuples, and none of the excluded ones. We have established a dichotomy for Unions of Conjunctive Queries or, equivalently, for $\forall \mathrm{CNF}$ formulas. For a special case, called final queries of type I we have also established a dichotomy for the model counting problem; this complements a result by Amarilli et al. [1] that prove a dichotomy for model counting for conjunctive queries without self-joins. We leave open the question whether UCQs admit a dichotomy for model counting.

\section{REFERENCES}

[1] Antoine Amarilli, Pierre Bourhis, and Pierre Senellart. Provenance circuits for trees and treelike instances. In Automata, Languages, and Programming - 42nd International Colloquium, ICALP 2015, Kyoto, Japan, July 6-10, 2015, Proceedings, Part II, pages 56-68, 2015. doi : 10.1007/978-3-662-47666-6\_5.

[2] Antoine Amarilli and Benny Kimelfeld. Model counting for conjunctive queries without self-joins. CoRR, abs/1908.07093, 2019. URL: http://arxiv.org/abs/1908. 07093, arXiv: 1908.07093.

[3] Paul Beame, Guy Van den Broeck, Eric Gribkoff, and Dan Suciu. Symmetric weighted first-order model counting. In Proceedings of the 34th ACM Symposium on Principles of Database Systems, PODS 2015, Melbourne, Victoria, Australia, May 31 - June 4, 2015, pages 313-328, 2015. doi : 10.1145/2745754.2745760.

[4] Nilesh N. Dalvi and Dan Suciu. The dichotomy of probabilistic inference for unions of conjunctive queries. F. ACM, 59(6):30:1-30:87, 2012. doi :10.1145/ 2395116.2395119

[5] Guy Van den Broeck, Wannes Meert, and Adnan Darwiche. Skolemization for weighted first-order model counting. In Principles of Knowledge Representation and Reasoning: Proceedings of the Fourteenth International Conference, KR 2014, Vienna, Austria, July 20-24, 2014, 2014. URL: http://www.aaai.org/ocs/index.php/ KR/KR14/paper/view/8012.

[6] Dan Geiger and Judea Pearl. Logical and algorithmic properties of independence and their application to bayesian networks. Ann. Math. Artif. Intell., 2:165-178, 1990. doi : 10.1007/BF01531004.

[7] Batya Kenig and Dan Suciu. A dichotomy for the generalized model counting problem for unions of conjunctive queries. CoRR, abs/2008.00896, 2020. URL: https://arxiv.org/abs/2008.00896, arXiv: 2008.00896.

[8] C. Krattenthaler. Advanced determinant calculus. In Dominique Foata and Guo-Niu Han, editors, The Andrews Festschrift, pages 349-426, Berlin, Heidelberg, 2001. Springer Berlin Heidelberg.

[9] J. Scott Provan and Michael O. Ball. The complexity of counting cuts and of computing the probability that a graph is connected. SIAM F. Comput., 12(4):777788, 1983. doi : $10.1137 / 0212053$.

[10] Richard P. Stanley. Enumerative Combinatorics: Volume 1. Cambridge University Press, USA, 2nd edition, 2011.

[11] Dan Suciu, Dan Olteanu, Christopher Ré, and Christoph Koch. Probabilistic Databases. Synthesis Lectures on Data Management. Morgan \& Claypool Publishers, 2011. doi : 10.2200/S00362ED1V01Y201105DTM016. 


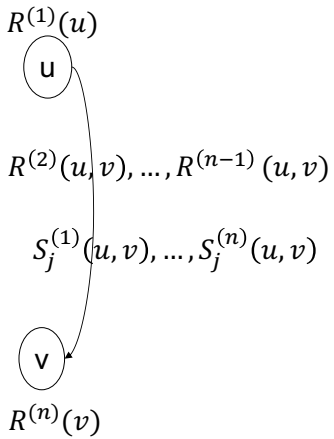

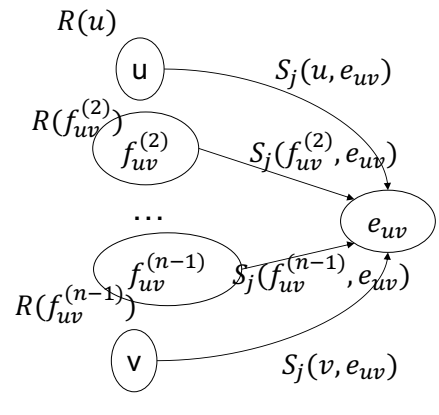

$R(v)$

\begin{abstract}
$\Delta$
$\mathrm{zg}(\Delta)$

Figure 2: Illustration for a reduction $\operatorname{PQE}(z g(Q)) \leq_{m}^{P} \mathrm{PQE}(Q)$ where $Q$ is a Type I-II query. Given a query $Q$ we construct the query $\mathrm{zg}(Q)$. Then, given the database $\Delta$ for $\mathrm{zg}(Q)$ on the left, we construct the database $\mathrm{zg}(\Delta)$ for $Q$ on the right. Here $n$ is one plus the longest right clause of $Q$; for example if $Q_{\text {right }}=\forall y\left(\forall x S_{1}(x, y) \vee \forall x S_{2}(x, y)\right.$, then $n=3$ and there is a single node $f_{u v}^{(2)}$, so that in total there are three edges incoming to $e_{u v}$.
\end{abstract}

\title{
APPENDIX
}

\section{A PROOF OF LEMMA 2.6}

Let $Q$ be an unsafe, bipartite query of length $k$, and let $C_{0}, C_{1}, \ldots, C_{k}$ be a minimal left-right path. Let $\mathcal{R}$ denote its vocabulary. We describe (a) a new unsafe, bipartite query $\mathrm{zg}(Q)$ of length $2 k$, over a new vocabulary $\mathrm{zg}(\mathcal{R})$ and (b) a polynomial-time mapping that takes a bipartite TID $\Delta$ over the vocabulary $\mathrm{zg}(\mathcal{R})$ and returns bipartite TID zg $(\Delta)$ over the vocabulary $\mathcal{R}$ such that $\operatorname{Pr}_{\Delta}(Q)=\operatorname{Pr}_{\mathrm{zg}(\Delta)}(\mathrm{zg}(Q))$ and $\mathrm{zg}(Q)$ is long. The probabilities values in $\Delta$ and $\mathrm{zg}(\Delta))$ are the same, which proves that $\operatorname{GFOMC}_{\mathrm{bi}}(\mathrm{zg}(Q)) \leq_{m}^{P} \mathrm{GFOMC}_{\mathrm{bi}}(Q)$. Moreover, if $Q$ is of type $A-B$, then $\mathrm{zg}(Q)$ is of type $A-A$. These properties prove Lemma 2.6.

Define a number $n$ as follows. If $Q_{\text {right }}$ is of Type I, then $n=2$. Otherwise, recall from Def. 2.3 that the right clauses of type II have the form $\forall y\left(\bigvee_{i=1}^{\ell}\left(\forall x S_{J_{\ell}}(x, y)\right)\right)$. We define $n$ to be the maximum between 3 , and the largest value of $\ell$ of all right clauses. Thus, by definition $n \geq 3$.

The vocabulary $\mathbf{z g}(\mathcal{R})$. We start by describing the mapping zg between the vocabularies. The vocabulary $\mathcal{R}$ contains a set of binary symbols $S_{1}, \ldots, S_{t}$. In addition, it may contain a single unary symbol $R$, and a single unary symbol $T$. The new vocabulary zg $(\mathcal{R}) \operatorname{consists}$ of $m+n$ disjoint copies of $\mathcal{R}$, as follows:

- If $R \in \mathcal{R}$ then $\operatorname{zg}(\mathcal{R})$ contains symbols $R^{(1)}, R^{(2)}, \ldots, R^{(n)}$, where the first and last one are unary, and all others are binary.

- For every binary symbol $S \in \mathcal{R}, \mathrm{zg}(\mathcal{R})$ contains the following binary symbols: $S^{(1)}, \ldots, S^{(n)}$.

- If $T \in \mathcal{R}$, then $\operatorname{zg}(\mathcal{R})$ contains a binary symbol $T^{(12)}$.

Notice that the only unary symbols are $R^{(1)}$ and $R^{(n)}$; the symbol $T$ became a binary symbol $T^{(12)}$. These will be the unary symbols " $R$ and $T$ " of the new query $z g(Q)$. Thus, if $Q$ was of type I-I or I-II then $\operatorname{zg}(Q)$ will be of type I-I because it has the two unary symbols $R^{(1)}$ and $R^{(n)}$. If $Q$ was of type II-I or II-II, then it has no $R$-symbol, hence $\mathrm{zg}(Q)$ has no unary symbols, i.e. it will be of type II-II.

The database $\mathbf{z g}(\Delta)$. Next, given a bipartite probabilistic database $\Delta=($ Dom, $p)$ over vocabulary $\mathrm{zg}(\mathcal{R})$, we describe how to construct $\left(\mathrm{zg}(\Delta), p^{\prime}\right)$. Since $\Delta$ is bipartite, is domain is Dom $=V_{1} \cup V_{2}$ and the only tuples $t$ with $p(t) \neq 1$ are those of the form $S_{j}(u, v), u \in V_{1}, v \in V_{2}$. Define the following bipartite database, $\operatorname{zg}(\Delta)=\left(\mathrm{Dom}^{\prime}, p^{\prime}\right)$ over the vocabulary $\mathcal{R}$. Its domain Dom ${ }^{\prime}=U_{1} \cup U_{2}$ consists of the following constants:

- For every $u \in V_{1}, u$ is in $U_{1}$.

- For every $v \in V_{2}, v$ is in $U_{1}$.

- For every pair $u \in V_{1}, v \in V_{2}$, there is a fresh element denoted $e_{u v}$, which is in $U_{2}$.

- For every pair $u \in V_{1}, v \in V_{2}$, there are $n-2$ fresh elements $f_{u v}^{(i)}, i=2, \ldots, n-1$, all of which are in $U_{1}$.

To define the tuple probabilities $p^{\prime}$ in $\Delta$, we establish a 1-to-1 correspondence between the tuples in $\Delta$ and those in $\mathrm{zg}(\Delta)$, which, in turn, defines $p^{\prime}$ in terms of $p$ :

$$
\text { - } p^{\prime}(R(u)) \stackrel{\text { def }}{=} p\left(R^{(1)}(u)\right) \text { for all } u \in V_{1} \text {. }
$$


- $p^{\prime}\left(R\left(f_{u v}^{(i)}\right)\right)=p\left(R^{(i)}(u, v)\right)$ for all $u \in V_{1}, v \in V_{2}, i \in[2, n-1]$.

- $p^{\prime}(R(v)) \stackrel{\text { def }}{=} p\left(R^{(n)}(v)\right)$ for all $v \in V_{2}$.

- $p^{\prime}\left(S\left(u, e_{u v}\right)\right) \stackrel{\text { def }}{=} p\left(S^{(1)}(u, v)\right)$ for all $u \in V_{1}, v \in V_{2}$.

- $p^{\prime}\left(S\left(f_{u v}^{(i)}, e_{u v}\right)\right) \stackrel{\text { def }}{=} p\left(S^{(i)}(u, v)\right)$ for all $u \in V_{1}, v \in V_{2}, i=2, \ldots, n-1$.

- $p^{\prime}\left(S\left(v, e_{u v}\right)\right) \stackrel{\text { def }}{=} p\left(S^{(n)}(u, v)\right)$ for all $u \in V_{1}, v \in V_{2}$.

- $p^{\prime}\left(T\left(e_{u v}\right) \stackrel{\text { def }}{=} p\left(T^{(12)}(u, v)\right)\right.$

All other tuples have probability $p^{\prime}(t)=1$.

The querry $\mathbf{z g}(Q)$. Finally, we define the zig-zag query $\mathrm{zg}(Q)$.

- For every left clause $C$ in $Q$ there are $n$ clauses in $\mathrm{zg}(Q)$, of which the first is a left clause, the last is a right clause, and the rest are middle clauses. More precisely, if $C$ is of type I clause, $C=R(x) \vee S_{J}(x, y)$ then $\mathrm{zg}(Q)$ contains the following clauses:

$$
\begin{aligned}
& \forall x \forall y\left(R^{(1)}(x) \vee S_{J}^{(1)}(x, y)\right) \\
\forall & \forall y\left(R^{(i)}(x, y) \vee S_{J}^{(i)}(x, y)\right) \\
& \forall x \forall y\left(R^{(n)}(y) \vee S_{J}^{(n)}(x, y)\right)
\end{aligned} \quad i=2, \ldots, n-1
$$

If $C$ is of type II, $C=\forall x\left(\bigvee_{i=1}^{m} \forall y\left(S_{J_{i}}(x, y)\right)\right)$ then $\mathrm{zg}(Q)$ contains the following clauses:

$$
\begin{aligned}
& \forall x\left(\bigvee_{i=1}^{m} \forall y\left(S_{J_{i}}^{(1)}(x, y)\right)\right) \\
& \forall x \forall y\left(\bigvee_{i=1}^{m} S_{J_{i}}^{(j)}(x, y)\right) \\
& \forall y\left(\bigvee_{i=1}^{m} \forall x\left(S_{J_{i}}^{(n)}(x, y)\right)\right)
\end{aligned}
$$

- Every middle clause $C=S_{J}(x, y)$ in $Q$ becomes $n$ middle clauses in $\mathrm{zg}(Q)$ :

$$
\forall x \forall y S_{J}^{(i)}(x, y) \quad i=1, \ldots, n
$$

- Every right clause becomes several middle clauses. There are two cases. If the right part of $Q$ is of Type I, then every right clause has the form $C=\forall x \forall y\left(S_{J}(x, y) \vee T(y)\right)$. In that case recall that $n=2$ and there will be exactly two middle clauses in $\mathrm{zg}(Q)$ :

$$
\begin{aligned}
& \forall x \forall y\left(S_{J}^{(1)}(x, y) \vee T^{(12)}(x, y)\right) \\
& \forall x \forall y\left(S_{J}^{(2)}(x, y) \vee T^{(12)}(x, y)\right)
\end{aligned}
$$

If the right part of $Q$ is of Type II, then every right clause has the form $C=\forall y\left(\bigvee_{i=1}^{\ell} \forall x S_{J_{i}}(x, y)\right)$. In this case, we create $n^{\ell}$ middle clauses in $\mathrm{zg}(Q)$, as follows. For every function $\phi:[\ell] \rightarrow[n]$ there will be one middle clause (for a total of $n^{\ell}$ middle clauses):

$$
C^{(\phi)} \stackrel{\text { def }}{=} \forall x \forall y\left(S_{J_{1}}^{(\phi(1))}(x, y) \vee \cdots \vee S_{J_{\ell}}^{(\phi(l))}(x, y)\right)
$$

$\mathrm{zg}(Q)$ is defined as the conjunction of all clauses above. Recall that, by assumption, we minimize every $\forall \mathrm{CNF}$ expression, that means that the clauses described above need to be minimized, and redundant ones need to be removed.

We prove several properties of $\mathrm{zg}(Q)$ :

Lemma A.1. $\operatorname{Pr}_{\Delta}(\mathrm{zg}(Q))=\operatorname{Pr}_{\mathrm{zg}(\Delta)}(\mathrm{zg}(Q))$

Proof. We prove a stronger statement: the lineage of $Q$ on $\mathrm{zg}(\Delta)$ is equivalent to the lineage of $\mathrm{zg}(Q)$ on $\Delta$, up to the 1-to-1 correspondence between the tuples described above. For that, we will show that for each clause $C$ of $Q$, it's lineage is equivalent to the conjunction of lineages of clauses derived from $C$ in $\mathrm{zg}(Q)$. There are several cases. In all cases we exploit the fact that $x$ in $C$ can be mapped only to constants of the form $u, f_{u v}^{(2)}, \ldots, f_{u v}^{(n-1)}, v$, and $y$ can be mapped only to constants of the form $e_{u v}$, where $u \in V_{1}, v \in V_{2}$ :

- Suppose $C$ is a left clause. If it is of Type I, $C=R(x) \vee S_{J}(x, y)$, then its lineage (in $\left.z g(\Delta)\right)$ is the conjunction (over $u \in V_{1}$ and $v \in V_{2}$ ) of:

$$
\left(R(u) \vee S_{J}\left(u, e_{u v}\right)\right) \wedge \bigwedge_{i=2}^{n-1}\left(R\left(f_{u v}^{(i)}\right) \vee S_{J}\left(f_{u v}^{(i)}, e_{u v}\right)\right) \wedge\left(R(v) \vee S_{J}\left(v, e_{u v}\right)\right)
$$


This is precisely the lineage of the clauses (48)-(49). If $C$ is of type II, $C=\bigvee_{i=1}^{\ell} \forall y S_{J_{i}}(x, y)$ then its lineage is the conjunction of the following Boolean formulas:

$$
\begin{aligned}
& \bigwedge_{u \in V_{1}}\left(\bigvee_{i=1}^{\ell}\left(\bigwedge_{v \in V_{2}} S_{J_{i}}\left(u, e_{u v}\right)\right)\right) \\
& \bigwedge_{v \in V_{2}}\left(\bigvee_{i=1}^{\ell}\left(\bigwedge_{u \in V_{1}} S_{J_{i}}\left(v, e_{u v}\right)\right)\right) \\
& \bigwedge_{j=2}^{n-1}\left(\bigvee_{i=1}^{\ell} S_{J_{i}}\left(f_{u v}^{(j)}, e_{u v}\right)\right)
\end{aligned}
$$

which is precisely the lineage of the clauses (50)-(51).

- If $C$ is a middle clause, $C=S_{J}(x, y)$, then it's lineages in $\operatorname{zg}(\Delta)$ is the Boolean expression:

$$
S_{J}\left(u, e_{u v}\right) \wedge \bigwedge_{j \in[2, n-1]} S_{J}\left(f_{u v}^{(j)}, e_{u v}\right) \wedge S_{J}\left(v, e_{u v}\right)
$$

This is precisely the lineage of clause (52).

- If $C$ is a right clause, then we distinguish two cases. If it is of Type I, $C=S_{J}(x, y) \vee T(y)$, then recall that $n=2$, and its lineage is the conjunction over all $u, v$ of the expression:

$$
\left(S_{J}\left(u, e_{u v}\right) \vee T\left(e_{u v}\right)\right) \wedge\left(S_{J}\left(v, e_{u v}\right) \vee T\left(e_{u v}\right)\right)
$$

which is precisely the lineage of the expressions (53)-(54). If it is of Type II, $C=\forall y\left(\bigvee_{i=1}^{\ell} \forall x\left(S_{J_{i}}(x, y)\right)\right)$, then we note that $y$ must be mapped to some value $e_{u v}$. Fixing $y$ to $e_{u v}$ implies exactly $n$ possibilities for each $x$. Namely, $\left\{u, v, f_{u v}^{(2)}, \ldots, f_{u v}^{(n)}\right\}$. The lineage of $C$ is the conjunction, over all $u \in U_{1}, v \in V_{2}$, of the Boolean formula:

$$
\begin{aligned}
& \left(S_{J_{1}}\left(u, e_{u v}\right) \wedge S_{J_{1}}\left(f_{u v}^{(2)}, e_{u v}\right) \wedge \cdots S_{J_{1}}\left(f_{u v}^{(n-1)}, e_{u v}\right) \wedge S_{J_{1}}\left(v, e_{u v}\right)\right) \\
& \cdots \\
& \left(S_{J_{\ell}}\left(u, e_{u v}\right) \wedge S_{J_{\ell}}\left(f_{u v}^{(2)}, e_{u v}\right) \wedge \cdots S_{J_{\ell}}\left(f_{u v}^{(n-1)}, e_{u v}\right) \wedge S_{J_{\ell}}\left(v, e_{u v}\right)\right)
\end{aligned}
$$

which is equivalent to:

$$
\left(\bigwedge_{i \in \ell} S_{J_{1}}^{(i)}(u, v) \vee \cdots \vee \bigwedge_{i \in \ell} S_{J_{\ell}}^{(i)}(u, v)\right)
$$

We apply the distributivity law of $\vee$ over $\wedge$ to convert this expression into a CNF expression, and obtain the conjunction of all lineages of clauses (55).

This completes the proof.

Lemma A.2. If $Q$ is an unsafe query, then $\operatorname{zg}(Q)$ is also unsafe.

Proof. We start by observing that the following clauses in $\mathrm{zg}(Q)$ are both minimized and non-redundant:

- Every left clause of the form (48) or (50) is minimized and non-redundant.

- If $C$ is a middle clause in $Q$, then the middle clause $C^{(i)}$ in $\operatorname{zg}(Q)$ is minimized and non-redundant.

- If $C=\forall y \bigvee_{i=1}^{\ell} \forall x S_{J_{i}}(x, y)$ is a right clause in $Q$, then every middle clause $C^{(\phi)}$ of the form (55) where $\phi$ is injective (i.e. the indices $\phi(1), \phi(2), \ldots, \phi(\ell)$ are mapped to distinct elements of $[n])$ is minimized and non-redundant. Indeed, if there was some homomorhism $C_{0}^{\prime} \rightarrow\left(S_{J_{1}}^{(\phi(1))}(x, y) \vee S_{J_{2}}^{(\phi(2))}(x, y) \vee \cdots S_{J_{\ell}}^{(\phi(\ell))}(x, y)\right)$, where $C_{0}^{\prime}$ is a clause in $\mathrm{zg}(Q)$ derived from some clause $C_{0}$ in $Q$, then we can construct a homorphism $C_{0} \rightarrow\left(\forall x S_{J_{1}}(x, y) \vee \forall x S_{J_{2}}(x, y) \vee \ldots\right)$, implying that $C$ was redundant in $Q$, which is a contradiction. To see why, note that such a homomorphism would imply that $\operatorname{Symb}\left(C_{0}\right) \subseteq J_{i}$ for some $i \in[\ell]$. We remark here that, if $\phi$ is not injective, then $C^{(\phi)}$ may be redundant. Since we have chosen $n$ such that $n \geq \ell$, for for every right clause $C$ there exists some; injective $\phi$, and in that case $C^{(\phi)}$ is non-redundant. 
Example A.3. We justify here the reason for introducing the "dead end" branches $f_{u v}^{(i)}$. Consider $\operatorname{zg}(Q)$ where $Q$ is the following query:

$$
\begin{aligned}
Q & =\forall x \forall y\left(R(x) \vee S_{0}(x, y)\right) \\
& \wedge \forall x \forall y\left(S_{0}(x, y) \vee S_{1}(x, y)\right) \wedge \underbrace{\left(S_{1}(x, y) \vee S_{2}(x, y) \vee S_{3}(x, y)\right)}_{\stackrel{\text { def }}{=} D} \\
& \wedge \underbrace{\forall y\left(\forall x\left(U(x, y) \vee S_{1}(x, y) \vee S_{2}(x, y)\right) \vee \forall x\left(U(x, y) \vee S_{1}(x, y) \vee S_{3}(x, y)\right) \vee \forall x\left(U(x, y) \vee S_{2}(x, y) \vee S_{3}(x, y)\right)\right)}_{\stackrel{\text { def }}{=} C}
\end{aligned}
$$

The middle clause $D$ generates two clauses in $\mathrm{zg}(Q)$ :

$$
D^{(1)} \stackrel{\text { def }}{=}\left(S_{1}^{(1)}(x, y) \vee S_{2}^{(1)}(x, y) \vee S_{3}^{(1)}(x, y)\right) \quad D^{(2)} \stackrel{\text { def }}{=}\left(S_{1}^{(2)}(x, y) \vee S_{2}^{(2)}(x, y) \vee S_{3}^{(2)}(x, y)\right)
$$

Assuming $n=2$ (i.e. no "dead end" branches $\left.f_{u v}^{(i)}\right)$, the right clause $C$ is mapped to the conjunction of $2^{3}=8$ clauses $C^{(\phi)}$ and all become redundant. For example, when $\phi(1)=\phi(2)=1, \phi(3)=2$ then:

$$
C^{(\phi)}=\forall x \forall y\left(U^{(1)}(x, y) \vee S_{1}^{(1)}(x, y) \vee S_{2}^{(1)}(x, y) \vee S_{3}^{(1)}(x, y) \vee U^{(2)}(x, y) \vee S_{2}^{(2)}(x, y) \vee S_{3}^{(2)}(x, y)\right)
$$

And this is redundant because of $D^{(1)}$. It is easy to check that all 8 clauses $C^{(\phi)}$ are made redundant by either $D^{(1)}$ or $D^{(2)}$. We notice that the query $Q$ is even a forbidden query, to be discussed in Sec. B, where $U$ is called a ubiquitous symbol. Thus, the dead end branches cannot be avoided even if $Q$ were guaranteed to be a forbidden query.

Next, we prove that $\mathrm{zg}(Q)$ is an unsafe bipartite query, by showing the existence of a left-to-right path. By assumption $Q$ is an unsafe, bipartite query of length $k$, hence there exists a left-to-right path $C_{0}, C_{1}, \ldots, C_{k}$ in $Q$. We define a left-to-right path in zg $(Q)$ as follows. The path starts with:

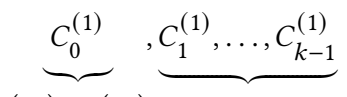

$$
(48) \text { or }(50) \quad(52)
$$

that is, we start with the translation of $C_{0}$ into a left clause $C_{0}^{(1)}$ using expression (48) if $C_{0}$ is of type I, or (50) if $C_{0}$ is of type II, then continue with the translations of the middle clauses, all using branch $i=1$. The path ends with the following:

$$
\underbrace{C_{k-1}^{(n)}, \ldots, C_{1}^{(n)}}_{(52)}, \underbrace{C_{0}^{(n)}}_{(49) \text { or }(51)}
$$

That is, it ends at the translation of $C_{0}$ into a right clause $C_{0}^{(n)}$, as per (49) or (51). So far, all clauses we have used are non-redundant. It remains to connect $C_{k-1}^{(1)}$ with $C_{k-1}^{(n)}$, and for that we use the right clause $C_{k}$. Assume first that $C_{k}$ is of type I, i.e. $C_{k}=\forall x \forall y\left(S_{J}(x, y) \vee T(y)\right)$; in that case $n=2$. By assumption $C_{k-1}$ and $C_{k}$ have some common symbol, call it $S$; obviously $S$ must be among the symbols $S_{J}$, since it cannot be $T$ (because $C_{k-1}$ is not a right clause). Define $C_{k}^{(1)}, C_{k}^{(2)}$ to be the middle clauses (53) and (54) respectively. The left-to-right path in $\mathrm{zg}(Q)$ is:

$$
C_{0}^{(1)}, C_{1}^{(1)}, \ldots, C_{k-1}^{(1)}, C_{k}^{(1)}, C_{k}^{(2)}, C_{k-1}^{(2)}, \ldots, C_{0}^{(2)}
$$

The clauses $C_{k-1}^{(1)}, C_{k}^{(1)}$ share the common symbol $S^{(1)}$; the clauses $C_{k}^{(1)}, C_{k}^{(2)}$ share the common symbol $T^{(12)}$, and the clauses $C_{k}^{(2)}, C_{k-1}^{(2)}$ share the common symbol $S^{(2)}$. Thus, the path above is a left-right path of length $2 k+1 \mathrm{in} z \mathrm{z}(Q)$. Assume now that $C_{k}$ is of type II, i.e. $C_{k}=\forall x S_{J_{1}}(x, y) \vee \cdots \forall x S_{J_{\ell}}(x, y)$ and let $S \in \operatorname{Symb}\left(C_{k-1}\right) \cap \operatorname{Symb}\left(C_{k}\right)$; assume wlog that $S \in \operatorname{Symb}\left(S_{J_{1}}\right)$. We consider two derived middle clause $C_{k}^{\left(\phi_{1}\right)}, C_{k}^{\left(\phi_{2}\right)}$ of the form (55), as follows. Both $\phi_{1}, \phi_{2}$ will be injective, ensuring that both clauses are non-redundant, and are defined as follows:

$$
\begin{array}{llr}
\phi_{1}(1) \stackrel{\text { def }}{=} 1 & \phi_{1}(2) \stackrel{\text { def }}{=} 2 & \phi_{1}:\{3, \ldots, \ell\} \rightarrow\{3, \ldots, n\} \text { any injective function } \\
\phi_{2}(1) \stackrel{\text { def }}{=} n & \phi_{2}(2) \stackrel{\text { def }}{=} 2 & \phi_{2}:\{3, \ldots, \ell\} \rightarrow\{1,3, \ldots, n-1\} \text { any injective function }
\end{array}
$$

Recall that we have defined $n \geq 3$, henc $\phi_{2}$ is injective. Thus, $C_{k}^{\left(\phi_{1}\right)}=\left(S_{J_{1}}^{(1)} \vee S_{J_{2}}^{(2)} \vee \cdots\right)$ and $C_{k}^{\left(\phi_{2}\right)}=\left(S_{J_{1}}^{(n)} \vee S_{J_{2}}^{(2)} \vee \cdots\right)$. Now we observe that $C_{k-1}^{(1)}, C_{k}^{\left(\phi_{1}\right)}$ share the common symbol $S^{(1)}$, the clauses $C_{k}^{\left(\phi_{1}\right)}, C_{k}^{\left(\phi_{2}\right)}$ share all common symbols in $S_{J_{2}}^{(2)}$, and $C_{k}^{\left(\phi_{2}\right)}, C_{k-1}^{(2)}$ share the common symbols $S^{(n)}$. Thus, the following is a left-to-right path in $\operatorname{zg}(Q)$ :

$$
C_{0}^{(1)}, C_{1}^{(1)}, \ldots, C_{k-1}^{(1)}, C_{k}^{\left(\phi_{1}\right)}, C_{k}^{\left(\phi_{2}\right)}, C_{k-1}^{(n)}, \ldots, C_{0}^{(n)}
$$


This proves that $\mathrm{zg}(Q)$ is an unsafe, bipartite query of length $\leq 2 k+1$.

Finally, it remains to prove that every left-to-right path in $\operatorname{zg}(Q)$ has length $\geq 2 k$, where $k$ is the length of the unsafe query $Q$. Consider any left-ot-right path in $\mathrm{zg}(Q): C_{0}^{\prime}, C_{1}^{\prime}, \ldots, C_{m}^{\prime}$. Since $C_{0}^{\prime}$ is a left clause it must be of the form (48) or (50), hence all its symbols are from the branch 1 , like $S^{(1)}$. Let $C_{\ell}^{\prime}$ be the first clause that contains an index of a branch other than 1, i.e. either some $S^{(i)}, i>1$ or $T^{(12)}$. $C_{\ell}^{\prime}$ must also have a symbol on branch 1 , because it shares a symbol with $C_{\ell-1}^{\prime}$ whose symbols are all on branch 1 , hence $C_{\ell}^{\prime}$ can only be of the form (53) or (55). Therefore, the fragment $C_{0}^{\prime}, C_{1}^{\prime}, \ldots, C_{\ell}^{\prime}$ of the path in $\mathrm{zg}(Q)$ corresponds to a left-to-right path $C_{0}, C_{1}, \ldots, C_{\ell}$ in $Q$, namely consisting of the clauses in $Q$ that generated the clauses $C_{0}^{\prime}, C_{1}^{\prime}, \ldots, C_{\ell}^{\prime}$ in $\operatorname{zg}(Q)$. Since $k$ is the length of the shortest left-right path in $Q$, we have $\ell \geq k$. We reason similarly about the suffix of the path. Since $C_{m}^{\prime}$ is a right clause, it is of the form (49) or (51) (note that it originates from a left clause $C_{m}$ ), hence all its symbols are on branch $n$, i.e. $S^{(n)}$. Let $C_{p}^{\prime}$ be the last clause that has some symbol on a branch other than $n$. Using the same argument as above, we conclude that $C_{p}^{\prime}$ originates from a right clause $C_{p}$, hence the fragment $C_{p}^{\prime}, C_{p+1}^{\prime}, \ldots, C_{m}^{\prime}$ of the path in $\mathrm{zg}(Q)$ corresponds to a left-to-right path in $Q$ (in reverse order): $C_{m}, C_{m-1}, \ldots, C_{p+1}, C_{p}$. Therefore, $m-p \geq k$. Since $\ell \leq p$ we conclude that $m \geq \ell+(m-p) \geq 2 k$.

\section{B BACKGROUND ON FACTORIZATION AND INDEPENDENCE}

Every multivariate polynomial $f$ admits a unique decomposition into irreducible factors: $f=g_{1}^{k_{1}} g_{2}^{k_{2}} \cdots g_{m}^{k_{m}}$. In this paper we use repeatedly the following:

THeorem B.1. Let $f_{00}, f_{01}, f_{10}, f_{11}$ be four multivariate polynomials, such that the following determinant is identically zero:

$$
\operatorname{det}\left(\begin{array}{ll}
f_{00} & f_{01} \\
f_{10} & f_{11}
\end{array}\right) \equiv 0
$$

Then, there exists polynomials $g_{0}, g_{1}$ and $h_{0}, h_{1}$ such that the following identity holds:

$$
\left[\begin{array}{l}
g_{0} \\
g_{1}
\end{array}\right] \cdot\left[\begin{array}{ll}
h_{0} & h_{1}
\end{array}\right] \equiv\left[\begin{array}{ll}
f_{00} & f_{01} \\
f_{10} & f_{11}
\end{array}\right]
$$

Proof. By induction on the total degree of $f_{00} f_{11}$. Let $p$ be any irreducible factor of $f_{00} f_{11} \equiv f_{01} f_{10}$. Assume that $p \mid f_{00}$ and $p \mid f_{01}$ (the other three cases are similar and omitted). Then the polynomials $f_{00} / p, f_{01} / p, f_{10}, f_{11}$ also satisfy the condition of the theorem, therefore, by induction hypothesis, there exists $g_{0}, g_{1}, h_{0}, h_{1}$ such that:

$$
\left[\begin{array}{l}
g_{0} \\
g_{1}
\end{array}\right] \cdot\left[\begin{array}{ll}
h_{0} & h_{1}
\end{array}\right] \equiv\left[\begin{array}{ll}
f_{00} / p & f_{01} \\
f_{10} / p & f_{11}
\end{array}\right]
$$

Then the polynomials $g_{0}, g_{1}, p h_{0}, h_{1}$ satisfy the condition of the theorem:

$$
\left[\begin{array}{l}
g_{0} \\
g_{1}
\end{array}\right] \cdot\left[\begin{array}{ll}
p h_{0} & h_{1}
\end{array}\right] \equiv\left[\begin{array}{ll}
f_{00} & f_{01} \\
f_{10} & f_{11}
\end{array}\right]
$$

The next two results concern variations on Lemma 1.1. We are given four monotone Boolean functions $F, G, H, K$ over $n$ variables, with arithmetizations $f, g, h, k$, and seek a valuation $\varphi \in\{0,1 / 2,1\}^{n}$ such that, on one hand $f[\varphi] g[\varphi] \neq h[\varphi] k[\varphi]$, on the other hand $\varphi$ avoids the value 0 as much as possible. We start with a simple case, when $\varphi$ avoids the value 0 completely.

If $\theta$ is a truth assignment of the Boolean variables of $F$, then we denote by $F_{\theta}$ the Boolean function obtained from $F$ by setting $X:=1$ for all variables where $\theta(X)=1$, and leaving the other variables free. For example, if $F\left(X_{1}, X_{2}, X_{3}, X_{4}\right)$ and $\theta\left(X_{1}\right)=\theta\left(X_{3}\right)=1, \theta\left(X_{2}\right)=\theta\left(X_{4}\right)=0$ then $F_{\theta} \stackrel{\text { def }}{=} F\left(1, X_{2}, 1, X_{4}\right)$. We denote by $\# F_{\theta}$ the number of models of the residual formula $F_{\theta}$.

Lemмa B.2. Let $F, G, H, K$ be four monotone Boolean functions with $n$ variables and $f, g, h, k$ their arithmetization. If $F \wedge G \not \equiv H \wedge K$ then there exists $\varphi \in\{1 / 2,1\}^{n}$ such that $f[\varphi] g[\varphi] \neq h[\varphi] k[\varphi]$.

Proof. Let $\theta$ be an assignment that distinguishes the two. Assume w.l.o.g. that:

$$
F[\theta]=0, \quad G[\theta] \in\{0,1\}, H[\theta]=K[\theta]=1
$$

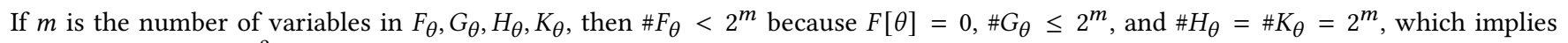
$\# H_{\theta} \# K_{\theta}-\# F_{\theta} \# G_{\theta}=2^{2 m}-\# F_{\theta} \# G_{\theta}>0$.

Corollary B.3. With the notations in Lemma B.2, if there exists any assignment $\varphi \in\{0,1 / 2,1\}$ such that $f[\varphi] g[\varphi] \neq h[\varphi] k[\varphi]$, then there exists an assignment $\varphi^{\prime} \in\{0,1 / 2,1\}$ such that $0 \neq f\left[\varphi^{\prime}\right] g\left[\varphi^{\prime}\right] \neq h\left[\varphi^{\prime}\right] k\left[\varphi^{\prime}\right] \neq 0$. 
Proof. If $f[\varphi] \neq 0, g[\varphi] \neq 0$, and $h[\varphi], k[\varphi] \neq 0$, then we are done. Otherwise, assume w.l.o.g. that $f[\varphi]=0$ while $h[\varphi], k[\varphi] \neq 0$. Let $\theta$ be the following assignment: $\theta(X)=0$ if $\varphi(X)=0$, and $\theta(X)=1$. We claim that $F[\theta]=0$. To see this, let $\theta^{\prime}$ be $\theta$ except the variables that are assigned $1 / 2$ in $\varphi$ remain unassigned. We now observe that $F_{\theta^{\prime}}(1 / 2, \ldots, 1 / 2)=c \cdot \# F_{\theta^{\prime}}$ where $c>0$. Since \#F $F_{\theta^{\prime}}=0$ then no assignment to the variables in the residual formula $F_{\theta^{\prime}}$ satisfies it, including the assignment that sets all of its variables to 1 .

So, we have that $F[\theta]=0$, and $H[\theta]=K[\theta]=1$, implying that $F \wedge G \not \equiv H \wedge K$, and we can use Lemma B.2.

When $F, G$ are equivalent to $H, K$ then obviously no $\varphi$ exists that satisfies Lemma B.2. But even when $F, G$ are different from $H, K$, such a $\varphi$ may still not exists, as illustrated by the following:

Example B.4. Consider the following four functions:

$$
\begin{array}{ll}
F=\left(X_{1} \vee X_{3}\right) \vee\left(Y_{1} \vee Y_{3}\right) \vee T & H=\left(X_{1} \vee X_{3}\right) \vee\left(Y_{2} \vee Y_{3}\right) \vee T \\
G=\left(X_{2} \vee X_{3}\right) \vee\left(Y_{2} \vee Y_{3}\right) \vee T & K=\left(X_{2} \vee X_{3}\right) \vee\left(Y_{1} \vee Y_{3}\right) \vee T \\
T=\left(Y_{1} \vee Y_{2} \vee Y_{3}\right)\left(X_{1} \vee X_{2} \vee X_{3}\right) &
\end{array}
$$

They are distinct, yet for any $\varphi \in\{1 / 2,1\}^{6}, f[\varphi] g[\varphi]=h[\varphi] k[\varphi]$. Indeed, if we set any variable $X_{i}$ to 1 then $F=H$ and $G=K$, if we set any

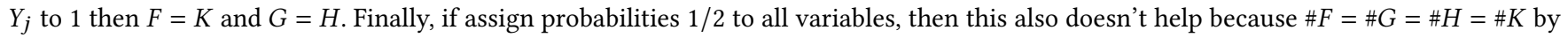
symmetry.

Lemma B.5. Let $F, G, H, K$ be monotone Boolean functions with $n$ variables, and let $U_{1}, \ldots, U_{k}$ be some Boolean variables with the property that $F\left[U_{i}:=1\right] \equiv G\left[U_{i}:=1\right] \equiv H\left[U_{i}:=1\right] \equiv K\left[U_{i}:=1\right]$, for all $i=1, k$. Assume that there exists $\varphi_{0} \in\{0,1 / 2,1\}$ such that $f\left[\varphi_{0}\right] g\left[\varphi_{0}\right] \neq$ $h\left[\varphi_{0}\right] k\left[\varphi_{0}\right]$. Then there exists $\varphi \in\{0,1 / 2,1\}$ such that $0 \neq f[\varphi] g[\varphi] \neq h[\varphi] k[\varphi] \neq 0$ and $\varphi\left(U_{i}\right) \in\{1 / 2,1\}$ for all $i=1, k$.

Proof. The multilinear polynomial $f$ admits the following expansion in terms of the variables $u_{1}, \ldots, u_{k}$ :

$$
f=\sum_{\tau \in\{0,1\}^{k}} \mathbf{u}^{\tau} f[\tau]
$$

where $\mathbf{u}^{\tau} \stackrel{\text { def }}{=} \prod_{i: \tau\left(u_{i}\right)=0}\left(1-u_{i}\right) \cdot \prod_{i: \tau\left(u_{i}\right)=1} u_{i}$. By assumption, for every $\tau \neq(0,0, \ldots, 0)$, we have $f[\tau]=g[\tau]=h[\tau]=k[\tau]$, thus we can write:

$$
\begin{aligned}
f & =\prod_{i}\left(1-u_{i}\right) f(0, \ldots, 0)+\Delta & h & =\prod_{i}\left(1-u_{i}\right) h(0, \ldots, 0)+\Delta \\
g & =\prod_{i}\left(1-u_{i}\right) g(0, \ldots, 0)+\Delta & k & =\prod_{i}\left(1-u_{i}\right) k(0, \ldots, 0)+\Delta
\end{aligned}
$$

where $\Delta$ is the same quantity for all four polynomials.

We can assume w.l.o.g. that $F \wedge G \equiv H \wedge K$, otherwise the claim follows immediately from Lemma B.2. We consider two cases. First, if $F \vee G \not \equiv H \vee K$. In that case we can assume w.l.o.g. that there exists a truth assignment $\theta$ such that $F[\theta]=G[\theta]=H[\theta]=0$ and $K[\theta]=1$; notice that $\theta\left(U_{i}\right)=0$ for all $U_{i}$ (otherwise $F[\theta]=K[\theta]$ ). Define $\varphi$ as follow: $\varphi\left(U_{i}\right)=1 / 2$ and $\varphi(X)=\theta(X)$ when $X \notin\left\{U_{1}, \ldots, U_{k}\right\}$. We first note that since $K$ is monotonic, then $k[\varphi]=1$. We compute $f[\varphi]$ using the formula above, noting that for $\tau=(0,0, \ldots, 0), f=0$, and for any other $\tau, f[\tau]=k[\tau]=1$ because $K[\theta]=1$. Therefore, $v \stackrel{\text { def }}{=} \prod\left(1-u_{i}\right)=1 / 2^{k}$ and $\Delta=\left(2^{k}-1\right) / 2^{k}=1-v$. We obtain:

$$
\begin{array}{rlrl}
f[\varphi] & =(1-v)>0 & & h[\varphi]=(1-v)>0 \\
g[\varphi]=(1-v)>0 & & k[\varphi]=1
\end{array}
$$

and we obtain $h[\varphi] k[\varphi]-f[\varphi] g[\varphi]>(1-v)-(1-v)^{2}>0$.

Second, assume that both identities hold: $F \wedge G \equiv H \wedge K$ and $F \vee G \equiv H \vee K$. Then $f+g \equiv h+k$ because:

$$
\operatorname{Pr}(F \vee G)=f+g-\operatorname{Pr}(F \wedge G)=\operatorname{Pr}(H \vee K)=h+k-\operatorname{Pr}(H \wedge K)
$$

Let $\varphi_{0}$ be an assignment such that $f\left[\varphi_{0}\right] g\left[\varphi_{0}\right] \neq h\left[\varphi_{0}\right] k\left[\varphi_{0}\right]$. Assume w.l.o.g. that $\varphi_{0}\left(U_{i}\right)=0$ for all $i$; otherwise, if $\varphi_{0}\left(U_{i}\right)=1 / 2$ then we simply remove the variable $U_{i}$ from the list $U_{1}, \ldots, U_{k}$ and decrease $k$. We define $\varphi$ as follows: $\varphi\left(U_{i}\right)=1 / 2$ for all variables $U_{i}$, and $\varphi(X)=\varphi_{0}(X)$ for all other variables. To prove the claim of the lemma, let $f_{0}, g_{0}, h_{0}, k_{0}$ be the polynomials in the variables $u_{1}, \ldots, u_{k}$ obtained by substituting all other variables $X$ with the value $\varphi_{0}(X)$. Thus, $f_{0}(0,0, \ldots, 0)=f\left[\varphi_{0}\right]$, and similarly for $g, h, k$, therefore,

$$
\begin{aligned}
k[\varphi] h[\varphi]-f[\varphi] g[\varphi] & =\left(\frac{1}{2^{k}} h_{0}(0, \ldots, 0)+\Delta\right)\left(\frac{1}{2^{k}} k_{0}(0, \ldots, 0)+\Delta\right)-\left(\frac{1}{2^{k}} f_{0}(0, \ldots, 0)+\Delta\right)\left(\frac{1}{2^{k}} g_{0}(0, \ldots, 0)+\Delta\right) \\
& =\frac{1}{2^{2 k}}\left(h\left[\varphi_{0}\right] k\left[\varphi_{0}\right]-f\left[\varphi_{0}\right] g\left[\varphi_{0}\right]\right)+\frac{\Delta}{2^{k}}\left(h\left[\varphi_{0}\right]+k\left[\varphi_{0}\right]-f\left[\varphi_{0}\right]-g\left[\varphi_{0}\right]\right) \\
& =\frac{1}{2^{2 k}}\left(h\left[\varphi_{0}\right] k\left[\varphi_{0}\right]-f\left[\varphi_{0}\right] g\left[\varphi_{0}\right]\right) \neq 0
\end{aligned}
$$

because $f+g=h+k$. Also, $\Delta>0$, because at least one of the four values $f\left[\varphi_{0}\right], g\left[\varphi_{0}\right], h\left[\varphi_{0}\right], k\left[\varphi_{0}\right]$ is $>0$, hence setting any $u_{i}:=1$ can only increase that value. This proves that $f[\varphi], g[\varphi], h[\varphi], k[\varphi]$ are $\neq 0$. 
Next, we discuss tight connections between Boolean formulas, their arithmetizations, and conditional independence statements, of possible independent interest.

Definition B.6. Fix a Boolean formula $F$.

- We say that $F$ is connected if, whenever $F \equiv F_{1} \wedge F_{2}$ where $F_{1}, F_{2}$ do not share any common Boolean variables, then either $F_{1} \equiv$ true or $F_{2} \equiv$ true. Otherwise we say that $F$ decomposes into $F_{1}, F_{2}$.

- We say that $F$ disconnects two sets of variables $\mathbf{U}, \mathbf{V}$, if $F \equiv F_{1} \wedge F_{2}$ where $F_{1}, F_{2}$ do not share any common Boolean variables, $\mathbf{V} \cap \operatorname{Vars}\left(F_{1}\right)=\emptyset, \mathbf{U} \cap \operatorname{Vars}\left(F_{2}\right)=\emptyset$. Otherwise, we say that $\mathbf{U}, \mathbf{V}$ are connected.

- A Boolean variable $X$ in $F$ disconnects $\mathbf{U}, \mathbf{V}$ if both $F[X:=0]$ and $F[X:=1]$ disconnect $\mathbf{U}, \mathbf{V}$.

Notice that, if $F$ does not depend on $\mathbf{U}$, then it trivially disconnects $\mathbf{U}, \mathbf{V}$, by writing $F=\operatorname{true} \wedge F$. We describe now the equivalent notions on multi-variate polynomials $f$.

Definition B.7. Let $f$ be a multivariate polynomial.

- We say that $f$ is irreducible if, whenever $f \equiv f_{1} \cdot f_{2}$, then either $f_{1}$ or $f_{2}$ is a constant polynomial.

- We say that $f$ disconnects two sets of variables $\mathbf{u}, \mathbf{v}$ if $f \equiv f_{1} \cdot f_{2}$ and $\mathbf{v} \cap \operatorname{Vars}\left(f_{1}\right)=\mathbf{u} \cap \operatorname{Vars}\left(f_{2}\right)=\emptyset$.

- We say that $x$ disconnects $\mathbf{u}, \mathbf{v}$ if both $f[x:=0]$ and $f[x:=1]$ disconnect $\mathbf{u}, \mathbf{v}$.

We prove that, if the arithmetization $f$ factorizes $f=g \cdot h$, then the associated Boolean function decomposes.

LEMmA B.8. Let $F$ be a Boolean formula, and assume its arithmetization factorizes as $f=g \cdot h$, where both $g$, $h$ are non-constant multi-linear polynomials. Then, there exists two Boolean formulas $G, H$ such that $F=G \wedge H$ and $g$, $h$ are, up to a constant, the arithmetization of $G$, $H$; in other words, there exists some constant $c \neq 0$ such that $\operatorname{Pr}(G)=c \cdot g$ and $\operatorname{Pr}(H)=h / c$.

Proof. We can assume w.l.o.g. that, $F \not \equiv$ false, and let $\theta$ be any assignment such that $F[\theta]=\operatorname{true}$. Then $f[\theta]=g\left[\theta_{1}\right] h\left[\theta_{2}\right]=1$, where $\theta_{1}, \theta_{2}$ are the restrictions of $\theta$ to $\operatorname{Vars}(g)$ and $\operatorname{Vars}(h)$ respectively. Denote $c=h\left[\theta_{2}\right] \neq 0$, then $g\left[\theta_{2}\right]=1 / c$. Define $G$, $H$ as follows. $\operatorname{Vars}(G)=\operatorname{Vars}(g), \operatorname{Vars}(H)=\operatorname{Vars}(h)$, and for any assignment $\theta_{1}^{\prime}$ of $\operatorname{Vars}(G)$, define $G\left[\theta_{1}^{\prime}\right] \stackrel{\text { def }}{=} c \cdot g\left[\theta_{1}^{\prime}\right]$ (similarly for $H$ ). We claim that this is correct, i.e. $c \cdot g\left[\theta_{1}^{\prime}\right] \in\{0,1\}$. Indeed, consider the full assignment $\theta^{\prime}=\theta_{1}^{\prime} \cup \theta_{2}$. If $F\left[\theta^{\prime}\right]=$ false then $0=f\left[\theta^{\prime}\right]=g\left[\theta_{1}^{\prime}\right] h\left[\theta_{2}\right]=c \cdot g\left[\theta_{1}^{\prime}\right]$, and if $F\left[\theta^{\prime}\right]=$ true then $1=f\left[\theta^{\prime}\right]=g\left[\theta_{1}^{\prime}\right] h\left[\theta_{2}\right]=c \cdot g\left[\theta_{1}^{\prime}\right]$, proving the claim. Thus, $\operatorname{Pr}(G)=c \cdot g$ and similarly $\operatorname{Pr}(H)=h / c$.

The following are easy to check:

Leмma B.9. Let $F$ be a Boolean formula and $f$ be the multilinear polynomial representing its arithmetization. Then:

- $F$ is connected iff $f$ is irreducible.

- $F$ disconnects $\mathbf{U}, \mathbf{V}$ iff $f$ disconnects $\mathbf{u}, \mathbf{v}$.

- $X$ disconnects $\mathbf{U}, \mathbf{V}$ iff $x$ disconnects $\mathbf{u}, \mathbf{v}$.

In this paper we are concerned only with monotone Boolean formulas $F$, which admit a unique, canonical CNF representation, where no clause is redundant (i.e. is not a superset of some other clause). Then, connectedness can be viewed as a graph-theoretic property, since it is equivalent to saying that there exists clauses $C_{0}, C_{1}, \ldots, C_{k}$ such that $\mathrm{U} \cap \operatorname{Vars}\left(C_{0}\right) \neq \emptyset, \mathrm{V} \cap \operatorname{Vars}\left(C_{k}\right) \neq \emptyset$ and $\operatorname{Vars}\left(C_{i-1}\right) \cap \operatorname{Vars}\left(C_{i}\right) \neq \emptyset$ for $i=1, k$. Define the distance, $d(\mathbf{U}, \mathbf{V})$, to be the minimum such $k$. Notice that we may have $d(U, V)=0$ even if $U, V$ are single variables. Connectedness is also an algebraic property, and related to polynomial factorization. We will make use repeatedly of these equivalent formulations.

Fix a set of variables $\mathbf{U}$. For any number $m \geq 0$, define the ball $B(\mathbf{U}, m) \stackrel{\text { def }}{=}\{Z \mid d(\mathbf{U}, Z) \leq m\}$. The following is easy to check:

Lemma B.10. Fix a monotone Boolean formula $F$.

(1) If $X$ disconnects $\mathrm{U}_{1}, \mathbf{V}$ and $X$ also disconnects $\mathrm{U}_{2}, \mathrm{~V}$, then it disconnects $\mathrm{U}_{1} \cup \mathrm{U}_{2}, \mathrm{~V}$.

(2) If $X$ disconnects $\mathbf{U}, \mathbf{V}$ and $m=d(\mathbf{U}, X), n=d(\mathbf{V}, X)$, then $X$ disconnects $B(\mathbf{U}, m-2)$ and $B(\mathbf{V}, n-2)$.

A third characterization uses conditional independence. Let $\operatorname{Pr}(-)$ denote the distribution where each random variable $X$ is set to true independently, with probability $\operatorname{Pr}(X)=x$. For a fixed Boolean formula $F$, define $\operatorname{Pr}_{F}$ the probability $\operatorname{space} \operatorname{Pr} \stackrel{\text { def }}{=} \operatorname{Pr}(-\mid F):$ that is, its outcomes are assignments that satisfy $F$. We write $\mathbf{U} \perp_{F} \mathbf{V}$ when $\mathbf{U}, \mathbf{V}$ are independent in the probability space $\operatorname{Pr}_{F}$. We prove the following:

Lemma B.11. A Boolean variable $X$ disconnects $\mathbf{U}, \mathbf{V}$, iff $\mathbf{U} \perp_{F} \mathbf{V} \mid X$.

Proof. We start by establishing the connection between $\operatorname{Pr}_{F}$ and the arithmetization $f$. Consider some partial assignment $\theta$ of the variables in $F$ with values in $\{0,1\}$. Then $\operatorname{Pr}_{F}(\theta)=f[\theta] / f$, where the polynomial $f$ is evaluated over values $x=\operatorname{Pr}(X=1), y=\operatorname{Pr}(Y=1), \ldots$ For example, if $F=X \vee Y$, then $\operatorname{Pr}(F)=f(x, y)=x+y-x y$ and $\operatorname{Pr}_{F}(X=1)=\operatorname{Pr}(X=1 \mid F)=f(1, y) / f(x, y)=1 /(x+y-x y)$. Assume first that $X$ disconnects $\mathbf{U}, \mathbf{V}$. Then $F=G \wedge H$ and $f=g \cdot h$, where $\mathbf{u} \subseteq \operatorname{Vars}(g)$, $\mathbf{v} \subseteq \operatorname{Vars}(h)$. For any assignments $\theta_{1}, \theta_{2}$ of the variables $\mathbf{U}, \mathbf{V}$ respectively, with values $\{0,1\}$ we write $\mathbf{U}=\theta_{1}$ for the event that the outcomes of $\mathbf{U}$ are those given by $\theta_{1}$. Then we have $\operatorname{Pr}_{F}\left(\mathbf{U}=\theta_{1}, \mathbf{V}=\theta_{2}\right)=f\left[\mathbf{U}=\theta_{1}, \mathbf{V}=\theta_{2}\right] / f=g\left[\mathbf{U}=\theta_{1}\right] \cdot h\left[\mathbf{V}=\theta_{2}\right] /(g \cdot h)=\operatorname{Pr}_{G}\left(\mathbf{U}=\theta_{1}\right) \cdot \operatorname{Pr}_{H}\left(\mathbf{V}=\theta_{2}\right)$. Conversely, assume 
$\operatorname{Pr}_{F}\left(\mathbf{U}=\theta_{1}, \mathbf{V}=\theta_{2}\right)=\operatorname{Pr}_{F}\left(\mathbf{U}=\theta_{1}\right) \operatorname{Pr}_{F}\left(\mathbf{V}=\theta_{2}\right)$. Then $f\left[\theta_{1}, \theta_{2}\right] / f=\left(f\left[\theta_{1}\right] / f\right) \cdot\left(f\left[\theta_{2}\right] / f\right)$, or, equivalently, $f\left[\theta_{1}, \theta_{2}\right] \cdot f=f\left[\theta_{1}\right] f\left[\theta_{2}\right]$. By Theorem B.1 there exists polynomials $g_{0}, g_{1}, h_{0}, h_{1}$ such that:

$$
f \equiv g_{0} h_{0} \quad f\left[\theta_{1}\right] \equiv g_{1} h_{0} \quad f\left[\theta_{2}\right] \equiv g_{0} h_{1} \quad f\left[\theta_{1} \theta_{2}\right] \equiv g_{1} h_{1}
$$

Since $f$ is multi-linear, $\operatorname{Vars}\left(g_{0}\right) \cap \operatorname{Vars}\left(h_{0}\right)=\emptyset$. From the first two identities we conclude that $\mathbf{u} \subseteq \operatorname{Vars}\left(g_{0}\right)$ (since, recall, $\theta_{1}$ assigns the variables $\mathbf{u}$ to 0,1$)$, and from identities one and three we conclude that $\mathbf{v} \subseteq \operatorname{Vars}\left(h_{0}\right)$. Thus, the factorization $f \equiv g_{0} h_{0}$ disconnects $\mathbf{u}$, $\mathbf{v}$, which proves the lemma.

Recall that, for every joint distribution of random variables, if $U_{1} U_{2} \perp V \mid X$ then $U_{1} \perp V \mid X$ and $U_{2} \perp V \mid X$. The converse does not hold in general, but it holds for $\perp_{F}$, by Lemma B.10.

Definition B.12. Let $F$ be a monotone Boolean formula, where $X$ disconnects $\mathbf{U}, \mathbf{V}$, and let $Y$ be any other variable in $F$. If $F$ does not disconnect either $\mathbf{U} Y, \mathbf{V}$, nor $\mathbf{U}, \mathbf{V} Y$, then we say that $Y$ is a migrating variable w.r.t. $X, \mathbf{U}, \mathbf{V}$.

Suppose $X$ disconnects $\mathbf{U}, \mathbf{V}$ in $F$, in other words $F[X=0]=G_{0} \wedge H_{0}$ and $F[X=1]=G_{1} \wedge H_{1}$, where $\operatorname{Vars}\left(G_{0}\right) \cap \operatorname{Vars}\left(H_{0}\right)=\emptyset$, $\operatorname{Vars}\left(G_{1}\right) \cap \operatorname{Vars}\left(H_{1}\right)=\emptyset$ and $\mathbf{U}$ occurs only in $G_{0}, G_{1}$ while $\mathbf{V}$ occurs only in $H_{0}, H_{1}$. Consider where $Y$ occurs. If it occurs in $G_{0}, G_{1}$ then $X$ separates $Y$ and $\mathbf{V}$; if it occurs in $H_{0}, H_{1}$ then $X$ separates $\mathbf{U}$ and $Y$. If none of these hold, then we say that it is migrating.

Lemma B.13. Assuming $m=d(\mathbf{U}, X), n=d(\mathbf{V}, X)$, if $Y$ migrates w.r.t. $X, \mathbf{U}, \mathbf{V}$, then $d(\mathbf{U}, Y) \geq m-1$, and $d(\mathbf{V}, Y) \geq n-1$.

Proof. Follows from Lemma B.10, item 2.

Example B.14. Consider the following Boolean function:

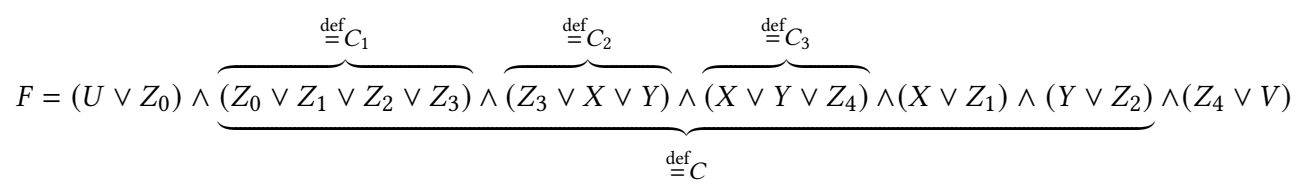

$X$ disconnects $U, V$, and we have:

$$
\begin{aligned}
& F[X:=0]= \overbrace{\left(U \vee Z_{0}\right) \wedge Z_{1}}^{\stackrel{\text { def }}{=} G_{0}} \overbrace{\left(Z_{3} \vee Y\right) \wedge\left(Y \vee Z_{4}\right) \wedge\left(Y \vee Z_{2}\right) \wedge\left(Z_{4} \vee V\right)}^{\stackrel{\text { def }}{=} H_{0}} \\
& F[X:=1]=\underbrace{\left(U \vee Z_{0}\right) \wedge\left(Z_{0} \vee Z_{1} \vee Z_{2} \vee Z_{3}\right) \wedge\left(Y \vee Z_{2}\right)}_{\stackrel{\text { def }}{=} G_{1}} \wedge \underbrace{\left(Z_{4} \vee V\right)}_{\stackrel{\text { def }}{=} H_{1}}
\end{aligned}
$$

Here $Y, Z_{2}$, and $Z_{3}$ migrate from the right to the left.

As we shall see, migrating variables add complexity to our proof. However, we prove that the migration property is symmetric: if $X$ causes $Y$ to migrate, then $Y$ causes $X$ to migrate. To prove this we use the following result of independent interest.

LEMma B.15. Let $X, Y, \mathbf{U}, \mathbf{V}$ be jointly distributed random variable, and assume that $Y$ is binary (i.e. it has only two outcomes). Then, the following implication holds: if $(\mathrm{U} \perp \mathrm{V} \mid X)$ and $(\mathrm{U} X \perp \mathrm{V} \mid Y)$ then either $(\mathrm{V} \perp Y)$ or $(\mathrm{U} \perp Y \mid X)$.

This implication does not hold in general, but it holds when $Y$ is binary. A similar, but different example is given by Geiger and Pearl [6], in Corollary 8.

Proof. Let $\operatorname{Pr}(-)$ denote the joint distribution. As usual we write $\operatorname{Pr}(X)$ or $\operatorname{Pr}(X Y)$ etc, for the marginal distribution. The first condition, $(\mathbf{U} \perp \mathbf{V} \mid X)$ says that $\operatorname{Pr}(\mathbf{U} X) \cdot \operatorname{Pr}(\mathbf{V} X)-\operatorname{Pr}(\mathbf{U V X}) \cdot \operatorname{Pr}(X)=0$. We use the fact that $Y$ has only two outcomes, and expand each probability, using the formula $\operatorname{Pr}(-)=\operatorname{Pr}(-\mid Y=0)(1-y)+\operatorname{Pr}(-\mid Y=1) y$, where $y \stackrel{\text { def }}{=} \operatorname{Pr}(Y=1)$, and further abbreviate $p_{0}(-)=\operatorname{Pr}(-\mid Y=0)$ and $p_{1}(-)=\operatorname{Pr}(-\mid Y=1)$, thus, for example $\operatorname{Pr}(\mathbf{U})=p_{0}(\mathbf{U})(1-y)+p_{1}(\mathbf{U}) y$. We also use the second condition, $(\mathbf{U} X \perp \mathbf{V} \mid Y)$, which implies $\operatorname{Pr}(\mathbf{U} X \mathbf{V} \mid Y)=\operatorname{Pr}(\mathbf{U} X \mid Y) \cdot \operatorname{Pr}(\mathbf{V} \mid Y)$ and $\operatorname{Pr}(\mathbf{V} X \mid Y)=\operatorname{Pr}(\mathbf{V} \mid Y) \cdot \operatorname{Pr}(X \mid Y)$, and derive:

$$
\begin{aligned}
0 & =\operatorname{Pr}(\mathbf{U} X) \cdot \operatorname{Pr}(\mathbf{V} X)-\operatorname{Pr}(\mathbf{U V X}) \cdot \operatorname{Pr}(X) \\
& =\left(p_{0}(\mathbf{U} X)(1-y)+p_{1}(\mathbf{U} X) y\right) \cdot\left(p_{0}(\mathbf{V} X)(1-y)+p_{1}(\mathbf{V} X) y\right)-\left(p_{0}(\mathbf{U V X})(1-y)+p_{1}(\mathbf{U V X}) y\right) \cdot\left(p_{0}(X)(1-y)+p_{1}(X) y\right) \\
& =\left(p_{0}(\mathbf{U} X)(1-y)+p_{1}(\mathbf{U} X) y\right) \cdot\left(p_{0}(\mathbf{V}) p_{0}(X)(1-y)+p_{1}(\mathbf{V}) p_{1}(X) y\right)-\left(p_{0}(\mathbf{U} X) p_{0}(\mathbf{V})(1-y)+p_{1}(\mathbf{U} X) p_{1}(\mathbf{V}) y\right) \cdot\left(p_{0}(X)(1-y)+p_{1}(X) y\right)
\end{aligned}
$$

We multiply out both products. The term $p_{0}(\mathrm{UX}) p_{0}(\mathbf{V}) p_{0}(X)(1-y)^{2}$ occurs in both products, and cancels out, and similarly for the term $p_{1}(\mathrm{U} X) p_{1}(\mathrm{~V}) p_{1}(X) y^{2}$, thus we obtain:

$$
\begin{aligned}
0 & =\left(p_{0}(\mathbf{U} X) p_{1}(\mathbf{V}) p_{1}(X) y(1-y)+p_{1}(\mathbf{U} X) p_{0}(\mathbf{V}) p_{0}(X) y(1-y)\right)-\left(p_{0}(\mathbf{U} X) p_{0}(\mathbf{V}) p_{1}(X) y(1-y)+p_{1}(\mathbf{U} X) p_{1}(\mathbf{V}) p_{0}(X) y(1-y)\right) \\
& =\left(p_{0}(\mathbf{U} X) p_{1}(X)-p_{1}(\mathbf{U} X) p_{0}(X)\right) \cdot\left(p_{1}(\mathbf{V})-p_{0}(\mathbf{V})\right) y(1-y)
\end{aligned}
$$


We can assume w.l.o.g. that $y(1-y) \neq 0$. If $\left(p_{1}(\mathbf{V})-p_{0}(\mathbf{V})\right)=0$ then $\mathbf{V} \perp Y$. Suppose $p_{1}(\mathbf{U} X) p_{0}(X)-p_{0}(\mathbf{U} X) p_{1}(X)=0$, then:

$$
\frac{p_{1}(\mathrm{U} X)}{p_{1}(X)}=\frac{p_{0}(\mathrm{U} X)}{p_{0}(X)}
$$

This is equivalent to $\operatorname{Pr}(\mathbf{U} \mid X, Y=1)=\operatorname{Pr}(\mathbf{U} \mid X, Y=0)$, or $\mathbf{U} \perp Y \mid X$.

We prove:

Corollary B.16. Let $F$ be a monotone, connected Boolean formula. Suppose $X$ disconnects $\mathrm{U}, \mathrm{V}$, and $Y$ also disconnects $\mathrm{U}$, V. Then $Y$ is migrating w.r.t. $X, \mathbf{U}, \mathbf{V}$ iff $X$ is migrating w.r.t. $Y, \mathbf{U}, \mathbf{V}$.

Proof. We prove the counterpositive: if $X$ does not migrate w.r.t. $Y, \mathrm{U}, \mathrm{V}$, then $Y$ does not migrate w.r.t. $X, \mathrm{U}, \mathrm{V}$. Since $X$ disconnects $\mathrm{U}, \mathbf{V}$, then, by Lemma B.11, we have $\mathbf{U} \perp_{F} \mathbf{V} \mid X$. If $X$ does not migrate w.r.t. $Y, \mathbf{U}, \mathbf{V}$, then $Y$ disconnects either $\mathbf{U} X, \mathbf{V}$ or $\mathbf{U}, \mathbf{V} X$. Assuming the former, we have $\mathbf{U} X \perp_{F} \mathbf{V} \mid Y$, Lemma B.15 implies that either $\left(\mathbf{V} \perp_{F} Y\right)$ or $\left(\mathbf{U} \perp_{F} Y \mid X\right)$ holds. The first is not possible because $F$ is connected, hence we have $\left(\mathrm{U} \perp_{F} \mathrm{Y} \mid X\right)$. Then we also have $\left(\mathrm{U} \perp_{F} \mathrm{~V} Y \mid X\right)$, proving that $Y$ does not migrate.

\section{PROOF OF THEOREM $2.9(2)$}

In this section we prove Theorem 2.9 (2): if $Q$ is a bipartite, unsafe query of type II of length $\geq 5$, then $\# P P 2 C N F \leq^{P}$ GFOMC $\mathrm{bi}(Q)$. For type I queries we proved that all probabilities in a block can be set to $1 / 2$, and therefore we obtained a symmetry (more precisely, we obtained $y_{01}=y_{10}$ ), allowing us to reduce from \#P2CNF. For type II queries we need to use all three probability values $0,1 / 2,1$ and may not have symmetries. This makes the proof a bit more complicated, and our reduction will be from \#PP2CNF rather than \#P2CNF.

Recall the definition of a bipartite query $Q$ in Def. 2.3. In this section $Q$ is of type II-II, and here we simply call it of type II. Recall the definition of a left-rigth path from Def. 2.4: it is a sequence of clauses $C_{0}, C_{1}, \ldots, C_{k}$ such that $C_{0}$ is a left clause, $C_{k}$ is a right clause, and every consecutive clauses share a common relational symbol. We will use repeatedly this simple fact:

Lemma C.1. Fix a left-to-right path $C_{0}, \ldots, C_{k}$. If $Q$ is a final query, then every symbol occurring in $Q$ must also occur in the left-to-right path.

Indeed, if a symbol $S$ does not occur on the path, then the query $Q[S:=1]$ still contains the path $C_{0}, \ldots, C_{k}$, since none of these clauses contained $S$, and therefore $Q[S:=1]$ is an unsafe query, contradicting the assumption that it is final.

\section{C.1 The Coloring Count Problem}

For Type I queries, our reduction was not directly from \#P2CNF, but from a more general problem, asking for all signature counts. Here, too, we need to consider a more general problem, which we define formally, and call it the Coloring Count Problem.

Fix two numbers $m \geq 2, n \geq 2$. For every bipartite graph $G=(U, V, E \subseteq U \times V)$, a coloring is a pair of functions $\sigma: U \rightarrow[m]$ and $\tau: V \rightarrow[n]$. The coloring associates a color to each node. Let $M \stackrel{\text { def }}{=} \max (|U|,|V|,|E|)$. Given a coloring $\sigma, \tau$, we denote by $k_{\alpha \beta}(\sigma, \tau)$ the number of edges whose endpoints are colored with $\alpha$ and $\beta$ respectively; denote by $k_{\alpha, \hat{1}}(\sigma, \tau)$ the number of nodes $u$ colored $\alpha$, and by $k_{\hat{1}, \beta}(\sigma, \tau)$ the number of nodes colored $\beta$. (The choice for the notation $\hat{1}$ will be come clear in the next section.) All these numbers are $\leq M$. Taking together, these numbers form the signature of a coloring $\sigma, \tau$, which is a mapping $\mathbf{k}(\sigma, \tau):([m] \cup\{\hat{1}\}) \times([n] \cup\{\hat{1}\}) \rightarrow\{0, \ldots, M\}$ where $k_{\hat{1}, \hat{1}}(\sigma, \tau) \stackrel{\text { def }}{=} 0$; equivalently, the signature is a vector with $(m+1)(n+1)$ dimensions and with values in $[0, M]$, i.e. $\in\{0, \ldots, M\}(m+1)(n+1)$. Formally:

$$
\begin{aligned}
\forall \alpha \in[m], \forall \beta \in[n]: & k_{\alpha \beta}(\sigma, \tau) \stackrel{\operatorname{def}}{=}|\{(u, v) \in E \mid \sigma(u)=\alpha, \tau(v)=\beta\}| \\
& k_{\alpha \hat{1}}(\sigma, \tau) \stackrel{\operatorname{def}}{=}|\{u \in U \mid \sigma(u)=\alpha\}| \\
& k_{\hat{1}, \beta}(\sigma, \tau) \stackrel{\operatorname{def}^{\prime}}{=}|\{v \in V \mid \tau(v)=\beta\}| \\
\text { Signature of } \sigma, \tau: & \mathbf{k}(\sigma, \tau) \stackrel{\text { def }}{=}\left(k_{\alpha, \beta}(\sigma, \tau)\right)_{\alpha \in[m] \cup\{\hat{1}\}, \beta \in[n] \cup\{\hat{1}\}}
\end{aligned}
$$

Conversely, given such a vector $\mathbf{k}:([m] \cup\{\hat{1}\}) \times([n] \cup\{\hat{1}\}) \rightarrow\{0, \ldots, M\}$, its coloring count, \#k, is the number of colorings $\sigma, \tau$ with signature $\mathbf{k}$ :

$$
\forall \mathbf{k} \in\{0, \ldots, M\}^{(m+1)(n+1)}: \quad \quad \# \mathbf{k} \stackrel{\operatorname{def}}{=}|\{(\sigma, \tau) \mid \mathbf{k}(\sigma, \tau)=\mathbf{k}\}|
$$

Definition C.2 (Coloring Counting Problem). We assume $m \geq 2, n \geq 2$ to be fixed. The Coloring Counting Problem, CCP $(m, n)$, is the following: given a bipartite graph $(U, V, E \subseteq U \times V)$, compute all coloring counts: $\{\# \mathbf{k} \mid \mathbf{k}:([m] \cup\{\hat{1}\}) \times([n] \cup\{\hat{1}\}) \rightarrow\{0, \ldots, M\}\}$, where $M=\max (|U|,|V|,|E|)$

Notice that the number of coloring counts is $(M+1)^{(m+1)(n+1)}$, hence, under the assumption that $m$ and $n$ are constant (in other words, $m, n=O(1))$, then the size of the output is polynomial in the size of the graph. 
Theorem C.3. For all $m, n \geq 2, \operatorname{CCP}(m, n)$ is \#P-hard.

Proof. Assuming we have an oracle for $\operatorname{CCP}(m, n)$, we describe a PTIME algorithm for computing \#PP2CNF. Let $\Phi=\bigwedge(u, v) \in E\left(X_{u} \vee Y_{v}\right)$ be a PP2CNF, where $E \subseteq U \times V$, and let $M=|E|$. Use the oracle to compute all coloring counts \#k, for all vectors $\mathbf{k}$. Call a coloring $\sigma, \tau$ valid if it uses only two colors, i.e. $\sigma(U) \subseteq\{1,2\}$ and $\tau(V) \subseteq\{1,2\}$, and call a signature $\mathbf{k}$ valid if $k_{\alpha \beta}=0$ whenever $\alpha>2$ or $\beta>2$. A valid coloring corresponds to a truth assignment, e.g. by associating false to color 1 and true to color 2 . The assignment satisfies $\Phi$ iff its signature satisfies $k_{11}=0$. Thus, \#Ф is the sum of \#k over all valid, satisfying signatures $\mathbf{k}$.

For example, assume we have 3 colors for the left, $a, b, c$ and 3 colors for the right, $u, v, w$. Then one can think of a vector $\mathbf{k}$ as a $4 \times 4$ matrix:

$$
\mathbf{k}=\left[\begin{array}{llll}
k_{a u} & k_{a v} & k_{a w} & k_{a \hat{1}} \\
k_{b u} & k_{b v} & k_{b w} & k_{b \hat{1}} \\
k_{c u} & k_{c v} & k_{c w} & k_{c \hat{1}} \\
k_{\hat{1} u} & k_{\hat{1} v} & k_{\hat{1} w} & k_{\hat{1} \hat{1}}
\end{array}\right]
$$

Given a bipartite graph with $M=|E|$ edges (we assume no isolated vertices), there are $M^{16}$ matrices $\mathbf{k}$. An oracle for the CCP(3,3) will compute all $M^{16}$ numbers \#k. To answer the \#PP2CNF formula, we consider only colorings that use the colors $a, b$ on the left, and $u, v$ on the right, e.g. $k_{c u}=k_{c v}=\ldots=0$. Using the counts \#k for these matrices, we can obtain \#PP2CNF.

In the rest of this appendix we prove:

THEOREM C.4. if $Q$ is a bipartite, unsafe query of type II-II of length $\geq 5$, then there exists numbers $m, n$ that depend only on $Q$ such that $\mathrm{CCP}(m, n) \leq^{P} \operatorname{GFOMC}_{b i}(Q)$

Theorem C.3 and Theorem C.4 prove Theorem 2.9 (2). In the rest of this appendix we prove Theorem C.4.

\section{C.2 Möbius Inversion formula for Type II Queries}

The principle behind the formula for Type I queries was the Shannon expansion formula: $\operatorname{Pr}(F)=\operatorname{Pr}(F[X:=0])(1-p)+\operatorname{Pr}(F[X:=1]) p$, where $X$ is a boolean variable and $p=\operatorname{Pr}(X)$. Essentially, we applied this formula repeatedly, once for each Boolean variable associated to a unary atom $R(u)$. For a Type II query, we no longer have unary atoms. Instead, we will use a different principle: the inclusion/exclusion formula, $\operatorname{Pr}(F \vee G)=\operatorname{Pr}(F)+\operatorname{Pr}(G)-\operatorname{Pr}(F \wedge G)$. We start by expressing $Q$ as a union, so we can enable the inclusion exclusion formula.

Recall the definition of a bipartite query $Q$ in Def. 2.3. $Q$ is of type II-II, and here we simply call it of type II. We will rewrite $Q$ as follows:

$$
\begin{aligned}
Q & =Q_{\text {left }} \wedge Q_{\text {middle }} \wedge Q_{\text {right }} \\
Q_{\text {left }} & =\forall x\left(\forall y G_{1}(x, y) \vee \ldots \vee \forall y G_{m}(x, y)\right) \stackrel{\text { def }}{=} \forall x G(x) \\
Q_{\text {middle }} & =\forall x \forall y C(x, y) \\
Q_{\text {right }} & =\forall y\left(\forall x H_{1}(x, y) \vee \ldots \vee \forall x H_{n}(x, y)\right) \stackrel{\text { def }}{=} \forall y H(y)
\end{aligned}
$$

where $m \geq 2, n \geq 2$, and $G_{i}(x, y), C(x, y), H_{j}(x, y)$ are CNF formulas, i.e. conjunctions of one or more clauses. Indeed, each type II query can be written this way, because $Q_{\text {left }}$ is a conjunction of left clauses, each of the form $\forall x\left(\bigvee_{\ell=1}^{m} \forall y S_{J_{\ell}}(x, y)\right)$, hence we obtain (57) by distributing $\wedge$ over $\vee$, in other words converting from CNF to DNF. We apply similar reason to $Q_{\text {right }}$ and obtain (59)

Example C.5. We illustrate this transformation on a $Q_{\text {left }}$ with two left clauses:

$$
\begin{aligned}
& Q_{\text {left }}=\underbrace{\forall x\left(\forall y\left(S_{1}(x, y) \vee S_{2}(x, y)\right) \vee\left(\forall y\left(S_{1}(x, y) \vee S_{3}(x, y)\right)\right)\right)} \wedge \underbrace{\forall x\left(\left(\forall y\left(S_{1}(x, y)\right)\right) \vee \forall y\left(S_{2}(x, y) \vee S_{3}(x, y)\right)\right)} \\
& \text { left clause } 1 \quad \text { left clause } 2 \\
& =\forall x\left(\forall y\left(S_{1}(x, y)\right) \vee \forall y\left(\left(S_{1}(x, y) \vee S_{2}(x, y)\right) \wedge\left(S_{2}(x, y) \vee S_{3}(x, y)\right)\right) \vee \forall y\left(\left(S_{1}(x, y) \vee S_{3}(x, y)\right) \wedge\left(S_{2}(x, y) \vee S_{3}(x, y)\right)\right)\right) \\
& \underbrace{=}_{\stackrel{\text { def }}{=} G_{1}(x, y)} \underbrace{S_{3}(x, y)}_{\stackrel{\text { def }}{=} G_{2}(x, y)} \\
& =\forall x\left(\forall y G_{1}(x, y) \vee \forall y G_{2}(x, y) \vee \forall y G_{3}(x, y)\right)
\end{aligned}
$$

Thus, we can write $Q$ as:

$$
Q=\left(\forall x \bigvee_{i} \forall y G_{i}\right) \wedge(\forall x \forall y C) \wedge\left(\forall y \bigvee_{j} \forall x H_{j}\right)
$$

which gets us closer to using the inclusion/exclusion formula. Here, each $G_{i}(x, y)$ and every $H_{j}(x, y)$ is a CNF formula, i.e. a conjunction of clauses. At this point we observe that some of the terms in the inclusion/exclusion formula can be logically equivalent. For example, given three Boolean formulas $F_{1}, F_{2}, F_{3}$, the expansion of $\operatorname{Pr}\left(F_{1} \vee F_{2} \vee F_{3}\right)$ has 7 terms, but some may be logically equivalent, e.g. we may have $F_{1} \wedge F_{2} \equiv F_{1} \wedge F_{2} \wedge F_{3}$. In our proof it is important to ensure that all terms are logically in-equivalent (we make this precise in Lemma C.16 below), and for that reason we consider next the lattice consisting of all logically inequivalent conjunctions: 
Definition C.6. Let $\mathbf{F}=\left\{F_{1}, \ldots, F_{m}\right\}$ be a set of formulas; For each set $\alpha \subseteq[m]$ denote by $F_{\alpha} \stackrel{\text { def }}{=} \bigwedge_{i \in \alpha} F_{i}$. The closure of $\alpha$ is: $\bar{\alpha} \stackrel{\text { def }}{=}\{i \mid$ $\left.F_{\alpha} \Rightarrow F_{i}\right\}$. A set $\alpha$ is closed if $\alpha=\bar{\alpha}$. The lattice associated with $\mathbf{F}$ denoted $\hat{L}(\mathbf{F})$ consists of all closed sets ordered by reverse set inclusion $\bar{\alpha} \leq \bar{\beta}$ if $\bar{\beta} \subseteq \bar{\alpha}$. We denote by $\hat{1}$ the top element of $\hat{L}(\mathbf{F})$ (that is, $\hat{1}=\emptyset$ ), and define $F_{\hat{1}} \stackrel{\text { def }}{=} F_{1} \vee \ldots \vee F_{m}$. The Möbius function $\mu: \hat{L}(\mathbf{F}) \rightarrow \mathbb{Z}$ is defined as $\mu(\hat{1})=1, \mu(\alpha)=-\sum_{\beta>\alpha} \mu(\beta)$. The support of $\mathbf{F}$ is $L(\mathbf{F}) \stackrel{\text { def }}{=}\{\alpha \in \hat{L}(\mathbf{F}) \mid \mu(\alpha) \neq 0\}$.

Intuitively, the lattice is obtained as follows. Compute all $2^{m}$ conjunctions $F_{\alpha}$, then group them into equivalence classes based on logical equivalence. The lattice consists of all equivalence classes. In each class there exists a maximal $\alpha$ such that $F_{\alpha}$ is in that class, and this $\alpha$ is closed; we use it as representative for the class. By convention, the top element of the lattice is defined as $F_{\hat{1}}=F_{1} \vee \cdots \vee F_{m}$; this is consistent to what we need in the rest of this section, and also standard in the context of the Möbius function, see [10, pp.117]. The support $L(\mathbf{F})$ is obtained by removing all elements $\alpha$ where $\mu(\alpha)=0$.

The Möbius inversion formula generalizes inclusion/exclusion, by combining equivalent terms. More precisely, if $\mathrm{Y}=\left\{Y_{1}, \ldots, Y_{m}\right\}$ is a set of $m$ formulas, then, Möbius' inversion formula is:

$$
\operatorname{Pr}\left(Y_{1} \vee \ldots \vee Y_{m}\right)=-\sum_{\alpha<\hat{1}} \mu(\alpha) \operatorname{Pr}\left(Y_{\alpha}\right)
$$

Obviously it suffices to sum only over the support, less $\hat{1}$, i.e. $\alpha \in L(\mathbf{F})-\{\hat{1}\}$, a fact that we will exploit later. As before, we write $y$ for the arithmetization of $Y$, i.e. the probability above expressed in terms of the probabilities of the Boolean variables, $z_{1}, z_{2}, \ldots$ Then:

$$
\operatorname{Pr}\left(y_{1} \vee \ldots \vee y_{m}\right)=-\sum_{\alpha<\hat{1}} \mu(\alpha) \cdot y_{\alpha}
$$

Example C.7. Consider the following set $\mathrm{Y}=\left\{Y_{1}, Y_{2}, Y_{3}\right\}$ :

$$
Y_{1}=Z_{1} Z_{2} \quad Y_{2}=Z_{1} Z_{3} \quad Y_{3}=Z_{2} Z_{3}
$$

where $Z_{1} Z_{2}$ means $Z_{1} \wedge Z_{2}$. We notice that $Y_{12}=Y_{13}=Y_{23}=Y_{123}=Z_{1} Z_{2} Z_{3}$ and therefore $\hat{L}(\mathrm{Y})=\{\emptyset, 1,2,3,123\}$. The Möbius function is $\mu(\emptyset)=1, \mu(1)=\mu(2)=\mu(3)=-1, \mu(123)=2$, thus the support is $\{\emptyset, 1,2,3,123\}$, and we obtain:

$$
\operatorname{Pr}\left(Z_{1} Z_{2} \vee Z_{1} Z_{3} \vee Z_{2} Z_{3}\right)=\operatorname{Pr}\left(Y_{1}\right)+\operatorname{Pr}\left(Y_{2}\right)+\operatorname{Pr}\left(Y_{3}\right)-2 \operatorname{Pr}\left(Y_{1} Y_{2} Y_{3}\right)
$$

For another example, consider $Y=\left\{Y_{1}, Y_{2}, Y_{3}\right\}$ where:

$$
Y_{1}=Z_{1} Z_{2} \quad Y_{2}=Z_{2} Z_{3} \quad Y_{3}=Z_{3} Z_{4}
$$

Then $\hat{L}(\mathrm{Y})=\{\emptyset, 1,2,3,12,23,123\}$, and $\mu(\emptyset)=1, \mu(1)=\mu(2)=\mu(3)=-1, \mu(12)=\mu(23)=1, \mu(123)=0$. The support consists of $\emptyset, 1,2,3,12,23$ and thus:

$$
\operatorname{Pr}\left(Y_{1} \vee Y_{2} \vee Y_{3}\right)=\operatorname{Pr}\left(Y_{1}\right)+\operatorname{Pr}\left(Y_{2}\right)+\operatorname{Pr}\left(Y_{3}\right)-\operatorname{Pr}\left(Y_{1} Y_{2}\right)-\operatorname{Pr}\left(Y_{2} Y_{3}\right)
$$

Given a bipartite query $Q$ of type II, we denote the following sets of formulas:

$$
\mathrm{G} \stackrel{\text { def }}{=}\left\{G_{1}(x, y) \wedge C(x, y), \ldots, G_{m}(x, y) \wedge C(x, y)\right\} \quad \quad \mathbf{H} \stackrel{\text { def }}{=}\left\{C(x, y) \wedge H_{1}(x, y), \ldots, C(x, y) \wedge H_{n}(x, y)\right\}
$$

where $G_{1}, \ldots, G_{m}, H_{1}, \ldots, H_{n}$ are the CNF formulas that occur in (57), (58), and (59) respectively. We define two lattices:

Definition C.8. Fix a bipartite, unsafe query query $Q$ of type II, as in Eq.(56). The left and right lattice supports of $Q$ are $L(\mathrm{G})$ and $L(\mathrm{H})$, where $\mathbf{G}$ and $\mathbf{H}$ are the sets defined above. The strict supports are $L_{0}(\mathbf{G}) \stackrel{\text { def }}{=} L(\mathbf{G})-\{\hat{1}\}$ and $L_{0}(\mathbf{H}) \stackrel{\text { def }}{=} L(\mathbf{H})-\{\hat{1}\}$, and we denote by $\bar{m}=\left|L_{0}(\mathbf{G})\right|$ and $\bar{n}=\left|L_{0}(\mathbf{H})\right|$ their sizes. Notice that $3 \leq \bar{m} \leq 2^{m}-1$ and $3 \leq \bar{n} \leq 2^{n}-1$.

We define the following, where $\alpha \in L(\mathbf{G})$ and $\beta \in L(\mathbf{H})$ :

$$
\begin{aligned}
G_{\alpha}(x) & \stackrel{\text { def }}{=} \forall y G_{\alpha}(x, y) \\
H_{\beta}(y) & \stackrel{\text { def }}{=} \forall x H_{\beta}(x, y) \\
Q_{\alpha \beta}(x, y) & \stackrel{\text { def }}{=} G_{\alpha}(x) \wedge Q \wedge H_{\beta}(y)
\end{aligned}
$$

We notice that, if $\alpha, \beta \neq \hat{1}$, then $\forall x \forall y Q_{\alpha \beta}(x, y)$ is equivalent to the following expression (recall that $Q_{\text {middle }}=\forall x \forall y C(x, y)$ ).

$$
\forall x \forall y Q_{\alpha \beta}(x, y)=\forall x \forall y\left(G_{\alpha}(x, y) \wedge C(x, y) \wedge H_{\beta}(x, y)\right)
$$

because, for every $\alpha \neq \hat{1}, \forall x G_{\alpha}(x) \Rightarrow Q_{\text {left. }}$. Indeed, each CNF expression $G_{i}$ in (60) is a conjunction of subclauses $S_{J_{k}}$, one from each left clause. Therefore, for any left clause $\forall x\left(\bigvee_{k} \forall y S_{J_{k}}(x, y)\right)$ in $Q_{\text {left }}$, the logical implication $G_{i}(x) \Rightarrow \bigvee_{k} \forall y S_{J_{k}}(x, y)$ holds, and therefore, $\forall x G_{\alpha}(x) \wedge Q_{\text {left }} \equiv \forall x G_{\alpha}(x)$ (since $\alpha \neq \emptyset$ ). On the other hand, if $\alpha=\hat{1}$, then:

$$
\forall x \forall y Q_{\hat{1} \beta}(x, y)=Q \wedge \forall y H_{\beta}(y) \quad \forall x \forall y Q_{\alpha \hat{1}}(x, y)=\forall x G_{\alpha}(x) \wedge Q
$$

because $Q_{\text {left }} \Rightarrow \forall x G_{\hat{1}}(x)$ and $Q_{\text {right }} \Rightarrow \forall y H_{\hat{1}}(y)$. 
Example C.9. Consider:

$$
Q=\forall x(\underbrace{\forall y S_{1}(x, y)}_{G_{1}(x, y)} \vee \underbrace{\forall y S_{2}(x, y)}_{G_{2}(x, y)}) \wedge \forall \forall y\left(S_{1}(x, y) \vee S_{3}(x, y)\right) \wedge \forall y(\underbrace{\forall x S_{3}(x, y)}_{H_{1}(x, y)} \vee \underbrace{\forall x S_{4}(x, y)}_{H_{2}(x, y)})
$$

Then:

$$
\begin{array}{llll}
G_{1}(x)=\forall y S_{1}(x, y) & G_{2}(x)=\forall y S_{2}(x, y) & G_{12}(x)=\forall y\left(S_{1}(x, y) \wedge S_{2}(x, y)\right) & G_{\hat{1}}(x)=\forall y\left(S_{1}(x, y) \vee S_{2}(x, y)\right) \\
H_{1}(y)=\forall x S_{3}(x, y) & H_{2}(y)=\forall x S_{4}(x, y) & H_{12}(x)=\forall x\left(S_{3}(x, y) \wedge S_{4}(x, y)\right) & H_{\hat{1}}(y)=\forall x\left(S_{3}(x, y) \vee S_{4}(x, y)\right)
\end{array}
$$

We show now a few examples of $Q_{\alpha \beta}$ :

$$
\begin{aligned}
\forall x \forall y Q_{1,1}(x, y) & =\forall x \forall y\left(S_{1}(x, y) \wedge S_{3}(x, y)\right) & & \forall x \forall y Q_{1,2}(x, y)=\forall x \forall y\left(S_{1}(x, y) \wedge S_{4}(x, y)\right) \\
\forall x \forall y Q_{1,12}(x, y) & =\forall x \forall y\left(S_{1}(x, y) \wedge S_{3}(x, y) \wedge S_{4}(x, y)\right) & & \forall x \forall y Q_{2,2}(x, y)=\forall x \forall y\left(S_{2}(x, y) \wedge\left(S_{1}(x, y) \vee S_{3}(x, y)\right) \wedge S_{4}(x, y)\right)
\end{aligned}
$$

Notice that the middle clause became redudant in all queries except $Q_{2,2}$.

\section{C.3 Forbidden Queries of Type II}

In our hardness proof we need all queries $Q_{\alpha \beta}$ to be connected: in example C.9 none of these queries is connected. To ensure this property, we need to restrict our queries to a strict subclass of final queries of Type II, which are called forbidden queries in [4]. Every final query of type II can be simplified to a forbidden query, hence it suffices to prove hardness for forbidden queries. In this section we give the formal definition of forbidden queries, prove the connectedness property, then prove that every final query of type II can be simplified to a forbidden query. The key results in this section are based on [4]. Here we expand those results and give a complete characterization of the forbidden queries. Our setting here is slightly simpler than that in [4], because we assume that the bipartite query $Q$ is long, i.e. the shortest left-right path has length $k \geq 2$.

Recall that a left clause is a union of subclauses $C(x)=\forall y S_{J_{1}}(x, y) \vee \forall y S_{J_{2}}(x, y) \vee \cdots$ A binary symbol $U(x, y)$ is $C$-ubiquitous if it occurs in all its subclauses $S_{J_{1}}, S_{J_{2}}, \ldots$ A binary symbol $U(x, y)$ is left ubiquitous if it is $C$-ubiquitous for all left clauses $C$. We define similarly right ubiquitous symbols, and denote them with $V$.

Definition C.10. Let $Q$ be a query of type II. $Q$ is called a forbidden query if it is a final query, and, for every left-right path $C_{0}, C_{1}, \ldots, C_{k}$ of minimal length, every symbol in $C_{0}$ is either ubiquitous, or occurs in $C_{1}$; similarly, every symbol in $C_{k}$ is either ubiquitous or occurs in $C_{k-1}$.

The following was shown in [4], and we included its proof here, later in this section:

THEOREM C.11. Let $Q$ be a final query of type II of length $k$, where $k \geq 2$. Then there exists a query $Q^{\prime}$ such that $\mathrm{GFOMC}_{b i}\left(Q^{\prime}\right) \leq_{m}^{P} \mathrm{GFOMC}_{b i}(Q)$ and $Q^{\prime}$ is either of type I, or $Q^{\prime}$ is a forbidden query of type II of length $\geq k$.

We also give a complete syntactic characterization of the forbidden queries, which is novel:

Theorem C.12. Let $Q=Q_{\text {left }} \wedge Q_{\text {middle }} \wedge Q_{\text {right }}$ be a forbidden query, and let $\mathbf{U}=\left\{U_{1}, \ldots, U_{r}\right\}, \mathbf{V}=\left\{V_{1}, \ldots, V_{t}\right\}$ denote the sets of leftubiquitous and right-ubiquitous symbols respectively, and let $C_{0}, C_{1}, \ldots, C_{k}$ be a left-to-right path of minimal length. Then, every clause in $Q$ has one of the following forms:

- A left clause, $\bigvee_{i} \forall y S_{J_{i}}(x, y)$, where each subclause $S_{J_{i}}$ is:

$$
U_{1}(x, y) \vee \cdots \vee U_{r}(x, y) \vee S_{J_{1}}(x, y) \vee S_{J_{2}}(x, y) \vee \cdots
$$

where $S_{J_{1}}, S_{J_{2}}, \ldots \subseteq \operatorname{Symb}\left(C_{1}\right)$. Notice that the left clause $C_{0}$ contains all left ubiquitous symbols.

- The middle clause $C_{1}$ does not contain any left-ubiquitous symbol.

- If $C$ is any middle clause that contains a left ubiquitous symbol, then $\operatorname{Symb}(C) \subseteq \operatorname{Symb}\left(C_{0}\right) \cup \operatorname{Symb}\left(C_{1}\right)$.

- A middle clause without any ubiquitous symbols, of the form $S_{j_{1}}(x, y) \vee S_{j_{2}}(x, y) \vee \cdots$.

- Symmetric clauses on the right.

Furthermore, if $r>1$, then for each $i=1, \ldots, r$ there exists at least one middle clause that contains $U_{i}$ and no other left ubiquitous symbol. Similarly, on the right.

Example C.13. We illustrate here a simple forbidden query:

$\forall x\left(\forall y\left(U(x, y) \vee S_{1}(x, y)\right) \vee \forall y\left(U(x, y) \vee S_{2}(x, y)\right)\right) \quad \wedge \forall x \forall y\left(S_{1}(x, y) \vee S_{2}(x, y) \vee S_{3}(x, y) \vee S_{4}(x, y)\right) \quad \wedge \forall y\left(\forall x\left(V(x, y) \vee S_{3}(x, y)\right) \vee \forall x\left(V(x, y) \vee S_{4}(x\right.\right.$

Here $U$ is a left-ubiquitous symbol, and $V$ is a right-ubiquitous symbol.

Example C.14. The ubiquitous symbols need not be unique, and may occur in middle clauses. Consider:

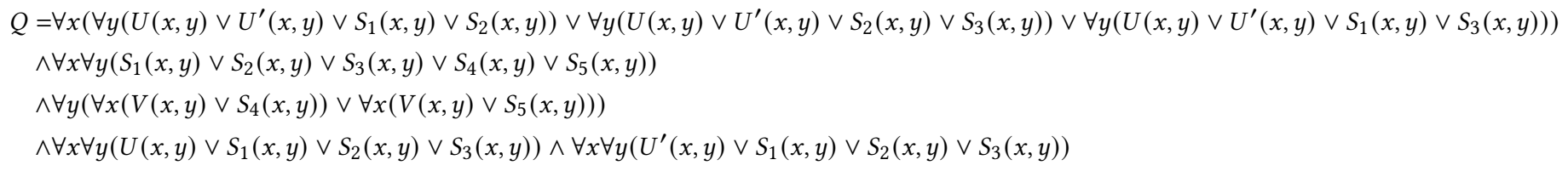


Before we prove the two theorems, we show the two consequences that will need later in this paper.

Lemma C.15 (Connected). Let $Q$ be a forbidden query. Then $\forall \alpha \in L(\mathrm{G}), \forall \beta \in L(\mathrm{H})$, the queries $\forall x \forall y Q_{\alpha \beta}(x, y)$ are connected, and depend on all relational symbols in $Q$.

Proof. Fix a left-to-right path $C_{0}, C_{1}, \ldots, C_{k}$ of minimal length; by Lemma C.1, all symbols in $Q$ occur on this path. Referring to the expressions in Eq. (64), all clauses $C_{1}, \ldots, C_{k-1}$ occur in $C(x, y)$. Assume first that $\alpha, \beta \neq \hat{1}$, then $\forall x \forall y Q_{\alpha \beta}(x, y)=\forall x \forall y\left(G_{\alpha}(x, y) \wedge C(x, y) \wedge\right.$ $\left.H_{\beta}(x, y)\right)$, and we prove that none of the clauses $C_{1}, \ldots, C_{k-1}$ becomes redundant. Recall that $G_{\alpha}(x, y)$ is a conjunction CNF expressions $G_{i}(x, y)$, each of which is a conjunction of subclauses $S_{J_{k}}(x, y)$ of some left clause of $Q$ (see Example C.5). Therefore every clause $S_{J_{k}}(x, y)$ of $G_{\alpha}(x, y)$ contains all ubiquitous symbols, while none of the clauses $C_{1}, \ldots, C_{k-1}$ contains all ubiquitous symbols, proving that none of the middle clauses on the minimal-length left-to-right path are not redundant. (In fact, by Theorem C.12, the middle clause $C_{1}$ does not contain any ubiquitous symbol. Since the path is minimal then none of the middle clauses contain any ubiquitous symbol.) Some clauses $S_{J_{k}}(x, y)$ of $G_{\alpha}(x, y)$ may become redundant, but the only homomorphisms $C^{\prime} \rightarrow S_{J_{k}}$ must be from some other clause $C^{\prime}$ of $G_{\alpha}(x, y)$ : otherwise, if $C^{\prime}$ is a middle clause, then we obtain a homomorphism $C^{\prime} \rightarrow C_{0}^{\prime}$, where $C_{0}^{\prime}$ is the left clause that contains $S_{J_{k}}$. Thus, at least one subclause of $G_{\alpha}(x, y)$ has to be non-redundant, proving that $Q_{\alpha \beta}$ depends on all relational symbols in $Q$. It remains to consider the cases $\alpha=\hat{1}$ or $\beta=\hat{1}$; assuming $\alpha=\hat{1}$, by Eq. (65) $\forall x \forall y Q_{\hat{1} \alpha}(x, y)=Q \wedge \forall y H_{\beta}(y)$ and the argument is similar.x

Lemma C.16 (Invertible). The mapping $(\alpha, \beta) \mapsto Q_{\alpha \beta}(x, y)$ is invertible. More precisely: if the logical implication $\forall x \forall y Q_{\alpha_{1}} \beta_{1}(x, y) \Rightarrow$ $\forall x \forall y Q_{\alpha_{2} \beta_{2}}(x, y)$ holds, then $\alpha_{1} \leq \alpha_{2}$ and $\beta_{1} \leq \beta_{2}$, in the lattices $\hat{L}(\mathrm{G})$ and $\hat{L}(\mathbf{H})$ respectively.

Proof. We expand $\forall x \forall y Q_{\alpha_{1} \beta_{1}} \Rightarrow \forall x \forall y Q_{\alpha_{2} \beta_{2}}$ and drop the quantifiers, to obtain:

$$
G_{\alpha_{1}}(x, y) \wedge C(x, y) \wedge H_{\beta_{1}}(x, y) \Rightarrow G_{\alpha_{2}}(x, y) \wedge C(x, y) \wedge H_{\beta_{2}}(x, y)
$$

Let $\mathbf{V}$ be all right ubiquitous symbols. If we set them to $\mathbf{V}:=1$, then $H_{\beta}[\mathbf{V}:=1]=1$ for every $\beta$, therefore we obtain:

$$
G_{\alpha_{1}}(x, y) \wedge C[\mathbf{V}:=1](x, y) \Rightarrow G_{\alpha_{2}}(x, y) \wedge C[\mathbf{V}:=1](x, y)
$$

We conjoin both terms with $C(x, y)$ and notice that $C[\mathbf{V}:=1] \wedge C \equiv C$ and therefore we obtain:

$$
G_{\alpha_{1}}(x, y) \wedge C(x, y) \Rightarrow G_{\alpha_{2}}(x, y) \wedge C(x, y)
$$

By definition this means $\alpha_{1} \leq \alpha_{2}$. We prove similarly that $\beta_{1} \leq \beta_{2}$.

We will now give the proof of Theorem C.11, but first illustrate the basic idea on an example.

Example C.17. The query $Q$ in Example C.9 is not forbidden; we repeat it here:

$$
Q=\forall x\left(\forall y S_{1}(x, y) \vee \forall y S_{2}(x, y)\right) \wedge \forall x \forall y\left(S_{1}(x, y) \vee S_{3}(x, y)\right) \wedge \forall y\left(\forall x S_{3}(x, y) \vee \forall x S_{4}(x, y)\right)
$$

Notice that $S_{2}$ does not occur in the middle clause. Define the following query, obtained by replacing $S_{2}$ with a unary symbol $R(x)$ :

$$
Q^{\prime}=\forall x \forall y\left(S_{1}^{\prime}(x, y) \vee R(x)\right) \wedge \forall x \forall y\left(S_{1}^{\prime}(x, y) \vee S_{3}^{\prime}(x, y)\right) \wedge \forall y\left(\forall x S_{3}^{\prime}(x, y) \vee \forall x S_{4}^{\prime}(x, y)\right)
$$

We claim that GFOMC $_{\mathrm{bi}}\left(Q^{\prime}\right) \leq_{m}^{P}$ GFOMC $_{\mathrm{bi}}(Q)$; since $Q^{\prime}$ is a query of Type I-II, we have already shown that GFOMC $\left(Q^{\prime}\right)$ is \#P-hard, and this implies that GFOMC $(Q)$ is also \#P-hard. To prove the claim, consider any probabilistic database $\Delta^{\prime}=\left(\right.$ Dom $\left.{ }^{\prime}, p^{\prime}\right)$ for $Q^{\prime}$. Define the following probabilistic database $\Delta=($ Dom, $p)$ for $Q$, where Dom $\stackrel{\text { def }}{=} \operatorname{Dom}^{\prime} \cup\left\{b_{1}\right\}$ for a fresh constant $b_{1}$, and where the probabilities are defined as follows, for all $a, b \in$ Dom:

$$
\begin{aligned}
& p\left(S_{2}\left(a, b_{1}\right)\right) \stackrel{\text { def }}{=} p^{\prime}(R(a)) \\
& j=1,3,4: p\left(S_{j}\left(a, b_{1}\right)\right) \stackrel{\text { def }}{=} 1 \\
& p\left(S_{2}(a, b)\right) \stackrel{\text { def }}{=} 1 \\
& p\left(S_{j}(a, b)\right) \stackrel{\text { def }}{=} p^{\prime}\left(S_{j}^{\prime}(a, b)\right)
\end{aligned}
$$

In $Q$, we have $\forall y S_{1}(x, y) \equiv\left(\forall y \neq b_{1} S_{1}(x, y)\right) \wedge S_{1}\left(x, b_{1}\right) \equiv \forall y S_{1}^{\prime}(x, y)$, where here the variable $y$ in $\forall y S_{1}^{\prime}(x, y)$ ranges over Dom', i.e. without $b_{1}$. Similarly, $\forall y S_{2}(x, y) \equiv\left(\forall y \neq b_{1} S_{2}(x, y)\right) \wedge\left(S_{2}\left(x, b_{1}\right)\right) \equiv R(x)$, etc, and the query $Q$ becomes:

$$
\begin{array}{rlll}
Q=\forall x\left(\forall y S_{1}(x, y) \vee \forall y S_{2}(x, y)\right) \wedge & & \forall x \forall y\left(S_{1}(x, y) \vee S_{3}(x, y)\right) \wedge & \forall y\left(\forall x S_{3}(x, y) \vee \forall x S_{4}(x, y)\right) \\
\equiv \forall x\left(R(x) \vee \forall y S_{1}^{\prime}(x, y)\right) \wedge & & \forall x \forall y\left(S_{1}^{\prime}(x, y) \vee S_{3}^{\prime}(x, y)\right) \wedge & \forall y\left(\forall x S_{3}^{\prime}(x, y) \vee \forall x S_{4}^{\prime}(x, y)\right)
\end{array}
$$

which is equivalent to $Q^{\prime}$, proving $\operatorname{Pr}(Q)=\operatorname{Pr}^{\prime}\left(Q^{\prime}\right)$.

Proof. (of Theorem C.11) Fix a left-right path in $Q$, not necessarily of minimal length, denote it $C_{0}, C_{1}, \ldots, C_{k}$, and recall that $C_{0}$ is a union of of subclauses $C_{0}=\forall x\left(\forall y S_{J_{1}}(x, y) \vee \forall y S_{J_{2}}(x, y) \vee \cdots\right)$. We start with the following:

Claim 1 (VARIANT of Lemma $8.36 \mathrm{IN}$ [4]). Suppose $S_{1}$ is a symbol that occurs in both $C_{0}, C_{1}, S_{0}$ is a symbol that occurs in $C_{0}$ and does not occur in $C_{1}, C_{2}, \ldots, C_{k}$. Then, if there exists a subclause $S_{J}(x, y)$ of $C_{0}$ that contains $S_{1}$ but not $S_{0}$, then there exists an unsafe query $Q^{\prime}$ with strictly fewer binary symbols such that $\mathrm{GFOMC}_{b i}\left(Q^{\prime}\right) \leq_{m}^{P} \mathrm{GFOMC}_{b i}(Q)$. 
The intuition is that, since $S_{0}$ only occurs in $C_{0}$, it should be a ubiquitous symbol, but fails to be one; then we can simplify $Q$ to $Q^{\prime}$. The query $Q^{\prime}$ will have left clauses that are slightly more general than those introduced in Definition 2.3: it may contain left clauses of the form:

$$
\forall x\left(R_{1}(x) \vee R_{2}(x) \vee \cdots \vee \forall y S_{J_{1}}(x, y) \vee \forall y S_{J_{2}}(x, y) \vee \cdots\right)
$$

Its middle and right clauses are as given in Definition 2.3. That is, its left clauses may contain multiple unary symbols and/or multiple subclauses. Such left clauses can be further simplified to either Type I left clauses, or Type II leff clauses, see Propositions 8.6 and 8.7 in [4].

Proof. (Of Claim 1) Let $S_{J_{1}}, \ldots, S_{J_{t}}$ be all the subclauses of $C_{0}$ that contain $S_{0}$; by assumption there exists at least one other sub-clause that contains $S_{1}$ and not $S_{0}$. Define $Q^{\prime}$ the query obtained from $Q$ as follows. The vocabulary consists of (a) for every symbol $S_{j}$ in $Q$ other than $S_{0}$, there is a fresh binary symbol $S_{j}^{\prime}(x, y)$ in $Q^{\prime}$. (b) for every $q=1, \ldots, t$ and every symbol $S_{j}$ that occurs in the subclause $S_{J_{q}}$ (including $\left.S_{0}\right)$ there is a fresh unary symbol denoted $R_{j}^{(q)}(x)$. Notice that $Q^{\prime}$ has one less binary symbol, since there is no $S_{0}^{\prime}(x, y)$. For any subclause $S_{J_{0}}(x, y)=S_{j_{1}}(x, y) \vee S_{j_{2}}(x, y) \vee \cdots \vee S_{j_{m}}(x, y)$ that occurs anywhere in $Q$, we denote the following expressions:

$$
S_{J_{0}}^{\prime} \stackrel{\text { def }}{=} S_{j_{1}}^{\prime}(x, y) \vee \cdots \vee S_{j_{m}}^{\prime}(x, y) \quad R_{J_{0}}^{(q)}= \begin{cases}R_{j_{1}}^{(q)}(x) \vee \cdots \vee R_{j_{m}}^{(q)}(x) & \text { if } \operatorname{Symb}\left(S_{J_{0}}\right) \subseteq \operatorname{Symb}\left(S_{J_{q}}\right) \\ \operatorname{true} & \text { otherwise }\end{cases}
$$

We construct the query $Q^{\prime}$ from $Q$ by replacing each subclause $S_{J_{0}}(x, y)$ with a new expression, according to the following two cases (the justification will become clear below, when we describe the mapping from $\Delta$ to $\Delta^{\prime}$ ):

Case 1: $S_{0} \in \operatorname{Symb}\left(S_{J_{0}}\right)$. Then replace $S_{J_{0}}(x, y)$ with $\bigwedge_{q=1, t} R_{J_{0}}^{(q)}(x)$. (Note that this might be true.)

Case 2: $S_{0} \notin \operatorname{Symb}\left(S_{J_{0}}\right)$. Then replace $S_{J_{0}}(x, y)$ with $\bigwedge_{q=1, t} R_{J_{0}}^{(q)}(x) \wedge S_{J_{0}}^{\prime}(x, y)$

We show now that $\operatorname{GFOMC}_{\mathrm{bi}}\left(Q^{\prime}\right) \leq_{m}^{P} \operatorname{GFOMC}_{\mathrm{bi}}(Q)$. Given a database $\Delta^{\prime}=\left(\operatorname{Dom}^{\prime}, p^{\prime}\right)$, we define (Dom $\stackrel{\text { def }}{=}$ Dom $\left.\cup\left\{b_{1}, b_{2}, \ldots, b_{t}\right\}, p\right)$, where $b_{1}, \ldots, b_{t}$ are fresh constants, and define:

$$
\begin{aligned}
& \forall q=1, t: p\left(S_{0}\left(a, b_{q}\right)\right) \stackrel{\text { def }}{=} p^{\prime}\left(R_{0}^{(q)}(a)\right) \\
& \forall q=1, t: \forall S_{j} \in \operatorname{Symb}\left(S_{J_{q}}\right)-\left\{S_{0}\right\} p\left(S_{j}\left(a, b_{q}\right)\right) \stackrel{\text { def }}{=} p^{\prime}\left(R_{j}^{(q)}(a)\right) \\
& \forall q=1, t: \forall S_{j} \in \operatorname{Symb}(Q)-\operatorname{Symb}\left(S_{J_{q}}\right) \quad p\left(S_{j}\left(a, b_{q}\right)\right) \stackrel{\text { def }}{=} 1 \\
& \begin{array}{l}
p\left(S_{0}(a, b)\right) \stackrel{\text { def }}{=} 1 \\
p\left(S_{j}(a, b)\right) \stackrel{\text { def }}{=} p^{\prime}\left(S_{j}^{\prime}(a, b)\right) \\
p\left(S_{j}(a, b)\right) \stackrel{\text { def }}{=} p^{\prime}\left(S_{j}^{\prime}(a, b)\right)
\end{array}
\end{aligned}
$$

We check that the lineage of $Q$ on $\Delta$ is the same as that of $Q^{\prime}$ on $\Delta^{\prime}$. Consider any subclause of $Q$ and assume first that it contains $S_{0}$ : $S_{J_{0}}(x, y)=S_{0}(x, y) \vee S_{j_{1}}(x, y) \vee \cdots$ When we substitute $y:=b_{q}$, then $S_{0}\left(x, b_{q}\right) \equiv R_{0}^{(q)}(x)$, and for every other symbol $S_{j_{i}}\left(x, b_{q}\right)$ is either $R_{j_{i}}^{(q)}(x)$ or true, hence the expression is equivalent to $R_{J_{0}}^{(q)}$; when we substitute $y:=b$ for some $b \in$ Dom', then $S_{0}(x, b) \equiv$ true and entire expression vanishes. This justifies Case 1 above. Case 2 is justified similarly and ommitted.

It remains to prove that $Q^{\prime}$ is an unsafe query. For that we prove that, once converted into $\mathrm{CNF}, Q^{\prime}$ has a left-right path of clauses that is non-redundant. We start by observing that every clause $C_{1}, C_{2}, \ldots, C_{k}$ on our path (other than $C_{0}$ ) is converted into an isomorphic clause, where each symbol $S_{j}(x, y)$ is replaced by $S_{j}^{\prime}(x, y)$. Indeed, by assumption, no subclause $S_{J_{0}}(x, y)$ on this path contains $S_{0}$, hence they are converted according to case 2. Moreover, $R_{J_{0}}^{(q)} \equiv$ true, because there must exist a symbol $S_{j}$ in $S_{J_{0}}$ that does not occur in $C_{0}$ : indeed, when $S_{J_{0}}$ is any middle clause $C_{i}, i=1, \ldots, k-1$, and $\operatorname{Symb}\left(C_{i}\right)=\operatorname{Symb}\left(S_{J_{0}}\right) \subseteq \operatorname{Symb}\left(S_{J}\right)$ then there is a homomorphism $C_{i} \rightarrow S_{J} \rightarrow C_{0}, \operatorname{contradiction}$. Since the length of $Q$ is $\geq 2, S_{J}$ has no common symbols with the right clause $C_{k}$. Consider any other clause $C$ of $Q$. Each of its subclauses $S_{J_{0}}$ is converted to a conjunction of up to $t+1$ expressions, i.e. either $\bigwedge_{q=1, t} R_{J_{0}}^{(q)}(x)$ or $\bigwedge_{q=1, t} R_{J_{0}}^{(q)}(x) \wedge S_{J_{0}}^{\prime}(x, y)$; notice that some expressions $R_{J_{0}}^{(q)}$ may be $\equiv$ true. We convert the resulting expression into CNF, thus from $C$ we obtain a conjunction $C^{\prime} \wedge C^{\prime \prime} \wedge \cdots$, each obtained by making one choice out of the up to $t+1$ choices for each subclause of $C$. Considering now $C_{0}$, we define $C_{0}^{\prime}$ to be obtained as follows: (a) for each subclause $S_{J_{q}}(x, y)$ of $C_{0}$, choose $R_{J_{0}}^{(q)}(x)$ (actually one can check that no other choice exists here) (b) for each other subclause $S_{J_{0}}$, choose $S_{J_{0}}^{\prime}$. That is, $C_{0}$ looks like this: $R_{J_{1}}^{(1)}(x) \vee R_{J_{1}}^{(2)}(x) \vee \cdots \vee R_{J_{t}}^{(t)}(x) \vee \forall y S_{J_{0}}^{\prime}(x, y) \vee \cdots$ By assumption there exists at least one subclause $S_{J_{0}}$ other than $S_{J_{1}}, \ldots, S_{J_{t}}$, hence $C_{0}^{\prime}$ is a left clause, i.e. it does not degenerate to $\vee_{q} R_{J_{q}}^{(q)}(x)$. (If we applied this construction to Example C.13 trying to remove $U(x, y)$, then the left clause degenerates to $R_{0}^{(1)}(x) \vee R_{1}^{(1)}(x) \vee R_{0}^{(2)}(x) \vee R_{2}^{(2)}(x)$.) Furthermore, the symbol $S_{1}^{\prime}$ is common in $C_{0}^{\prime}$ and $C_{1}^{\prime}$, hence $C_{0}^{\prime}, C_{1}^{\prime}, \ldots, C_{k}^{\prime}$ is a left-right path. It remains to prove that it is not redundant. For that, assume the contrary, that there exists a homomorphism $f: C^{\prime} \rightarrow C_{i}^{\prime}$, for some clause $C^{\prime} \not \equiv$ true. Let $C$ be the original clause from which we derived $C^{\prime}$ (thus $C$ became $\left.C^{\prime} \wedge C^{\prime \prime} \wedge \cdots\right)$, and consider any subclause $S_{J_{0}}(x, y)$ of $C$. We will construct a homomorphism $g: S_{J_{0}}(x, y) \rightarrow C_{i}$; by taking their union we obtain a homomorphism $C \rightarrow C_{i}$, contradicting the fact that $Q$ has no redundant clauses. To construct $g$, we consider the two cases above. Case 1: $S_{0} \in \operatorname{Symb}\left(S_{J_{0}}\right)$, then $C^{\prime}$ must contain some expression $R_{J_{0}}^{(q)}(x)$ for some choice of $q=1, \ldots, t$, and since $R_{J_{0}}^{(q)}(x) \not \equiv$ true (otherwise $C^{\prime} \equiv$ true), we have that $\operatorname{Symb}\left(S_{J_{0}}\right) \subseteq \operatorname{Symb}\left(S_{J_{q}}\right)$. Given the homomorphism $f: C^{\prime} \rightarrow C_{i}^{\prime}$, we have that $C_{i}^{\prime}$ contains $R_{J_{0}}^{(q)}(x)$, hence it must be 
that $i=0$. We simply define $g$ to map $S_{J_{0}}$ to the subclause $S_{J_{q}}$ of $C_{0}$. Case $2: S_{0} \notin \operatorname{Symb}\left(S_{J_{0}}\right)$. Its translation is $\wedge_{q=1, t} R_{J_{0}}^{(q)}(x) \wedge S_{J_{0}}^{\prime}(x, y)$, hence $C^{\prime}$ must contain either some $R_{J_{0}}^{(q)}(x)$, in which case we argue as in Case 1 , or contains $S_{J_{0}}^{\prime}(x, y)$. In that case we use the homomorphism $f$ : it maps $S_{J_{0}}^{\prime}(x, y)$ to $C_{i}^{\prime}$, we simply define $g$ similarly from $S_{J_{0}}^{\prime}(x, y)$ to $C_{i}$.

This concludes our proof of Claim 1 .

Example C.18. We illustrate with an example showing some of the subtleties of the proof of Claim 1. Consider two clauses $C_{0}, C_{1}$ that form the beginning of a left-right path. We apply Claim 1 to the symbol $S_{0}$ in $C_{0}$, noting that it does not co-occur with $S_{1}$, and show their conversions to $C_{0}^{\prime}, C_{1}^{\prime}$ below:

$$
\begin{array}{ll}
C_{0}=\forall x\left(\forall y S_{1}(x, y) \vee \forall y\left(S_{0}(x, y) \vee S_{2}(x, y)\right) \vee \forall y\left(S_{0}(x, y) \vee S_{3}(x, y)\right)\right) & C_{1}=\forall x \forall y\left(S_{1}(x, y) \vee S_{2}(x, y) \vee S_{4}(x, y)\right) \\
C_{0}^{\prime}=\forall x\left(\forall y S_{1}^{\prime}(x, y) \vee R_{0}^{(1)}(x) \vee R_{2}^{(1)}(x) \vee R_{0}^{(2)}(x) \vee R_{3}^{(2)}(x)\right) & C_{1}^{\prime}=\forall x \forall y\left(S_{1}^{\prime}(x, y) \vee S_{2}^{\prime}(x, y) \vee S_{4}^{\prime}(x, y)\right)
\end{array}
$$

There are two subclauses containing $S_{0}$. Thus, there are two unary symbols $R_{0}^{(1)}, R_{0}^{(2)}$ because $S_{0}$ occurs in both, and only one symbol $R_{2}^{(1)}$ and one symbol $R_{3}^{(2)}$ because $S_{2}, S_{3}$ occur only in one subclause respectively. We illustrate why $C_{0}^{\prime}, C_{1}$ ' do not become redundant, by considering several other clauses, which are not part of the left right path, and thus we denote them $D_{1}, D_{2}, \ldots$ :

$$
\begin{array}{ll}
D_{1}=\forall x\left(\forall y\left(S_{0}(x, y) \vee S_{1}(x, y)\right) \vee \forall y S_{2}(x, y)\right) & D_{1}^{\prime}=\forall x\left(\operatorname{true} \vee\left(R_{2}^{(1)}(x) \wedge \forall y S_{2}^{\prime}(x, y)\right)\right) \equiv \operatorname{true} \\
D_{2}=\forall x\left(\forall y\left(S_{1}(x, y) \vee S_{2}(x, y)\right) \vee \forall y S_{3}(x, y)\right) & D_{2}^{\prime}=\forall x\left(\forall y\left(S_{1}^{\prime}(x, y) \vee S_{2}^{\prime}(x, y)\right) \vee\left(R_{3}^{(2)}(x) \wedge \forall y S_{3}^{\prime}(x, y)\right)\right) \\
D_{3}=\forall x\left(\forall y S_{1}(x, y) \vee \forall y\left(S_{2}(x, y) \vee S_{3}(x, y)\right)\right) & D_{3}^{\prime}=\forall x\left(\forall y S_{1}^{\prime}(x, y) \vee \forall y\left(S_{2}^{\prime}(x, y) \vee S_{3}^{\prime}(x, y)\right)\right)
\end{array}
$$

Clause $D_{1}$ rewrites to true hence does not lead to any redundancies. Clause $D_{2}$ rewrites to the expression $D_{2}^{\prime}$, which further rewrites to two clauses: $\forall x\left(\forall y\left(S_{1}^{\prime}(x, y) \vee S_{2}^{\prime}(x, y)\right) \vee R_{3}^{(2)}(x)\right)$ and $\forall x\left(\forall y\left(S_{1}^{\prime}(x, y) \vee S_{2}^{\prime}(x, y)\right) \vee \forall y S_{3}^{\prime}(x, y)\right)$. Considering the first clause, we notice that there exists a homomorphism from $R_{3}^{(2)}(x)$ to $C_{0}^{\prime}$, but this is because there was a homomorphism from $\forall y S_{3}(x, y)$ to $\forall y\left(S_{0}(x, y) \vee S_{3}(x, y)\right)$; this does not extend to the entire clause (otherwise there would be a homomorphism $D_{2} \rightarrow C_{0}$ ). Finally, in $D_{3}$ we observe that the subclause $S_{J_{0}} \stackrel{\text { def }}{=} S_{2}(x, y) \vee S_{3}(x, y)$ rewrites to $S_{2}^{\prime}(x, y) \vee S_{3}^{\prime}(x, y)$, because $R_{J_{0}}^{(1)}(x) \equiv R_{J_{0}}^{(2)}(x) \equiv$ true since $S_{2}, S_{3}$ occur in separate clauses with $S_{0}$; one subtlety of the proof of the claim is precisely to ensure that and expression like this does not rewrite to $R_{2}^{(1)}(x) \wedge R_{3}^{(2)}(x) \wedge \forall y S_{3}^{\prime}(x, y)$, because after converting to $\mathrm{CNF}$ it will render $C_{0}^{\prime}$ redundant.

Continuing the proof of Theorem C.11, we can now assume w.l.o.g. that for every $S_{1}$ common to $C_{0}, C_{1}$ and for every $S_{0}$ occurring only in $C_{0}$, every subclause of $C_{0}$ containing $S_{1}$ must also contain $S_{0}$. Next we show:

Claim 2. Every subclause $S_{J}(x, y)$ of $C_{0}$ has some common symbol with $C_{1}$ (this is Lemma 8.38 in [4]).

Indeed, if $S_{J}^{\prime}$ is any other subclause of $C_{0}$ that does have some common symbol $S_{1}$ with $C_{1}$, then all symbols $S_{0}$ of $S_{J}$ must occur in $S_{J}^{\prime}$, therefore $\forall y S_{J}(x, y) \vee \forall y S_{J}^{\prime}(x, y) \equiv \forall y S_{J}(x, y)$, contradicting the assumption that $C_{0}$ has non redundant subclauses, proving claim 2 .

At this point we will restrict the left-right path $C_{0}, C_{1}, \ldots, C_{k}$ to be of minimal length.

Claim 3. Every symbol in $C_{0}$ is either $C_{0}$-ubiquitous, or occurs in $C_{1}$.

Proof. Let $S_{0} \in \operatorname{Symb}\left(C_{0}\right)-\operatorname{Symb}\left(C_{1}\right)$. By minimality, $S_{0}$ does not occur in $C_{1}, C_{2}, \ldots, C_{k}$. We prove that $S_{0}$ is $C_{0}$-ubiquitous. If it is not, then there exists a subclause $S_{J}$ that does not contain $S_{0}$. By Claim $2, S_{J}$ contains some symbol $S_{1}$ common with $C_{1}$, thus, by Claim $1 S_{J}$ must contain $S_{0}$, contradiction.

\section{Claim 4. There exists at least one $C_{0}$-ubiquitous symbol.}

Indeed, otherwise, all symbols of $C_{0}$ occur in $C_{1}$, which implies that there exists a homomorphism $C_{0} \rightarrow C_{1}$ (because $C_{1}$ is a middle clause), contradicting the fact that $Q$ has no redundant clauses; this proves the claim. Finally, we prove:

CLAIm 5. If $C_{0}, C_{0}^{\prime}$ are two left clauses then the $C_{0}$-ubiquitous symbols are the same as the $C_{0}^{\prime}$-ubiquitous symbols.

We first prove that $C_{0}^{\prime}$ shares some common symbols with $C_{1}$. Assuming otherwise, since all symbols in $Q$ must occur on the path $C_{0}, C_{1}, \ldots, C_{k}$, and this path has minimal length, it follows that all symbols of $C_{0}^{\prime}$ occur in $C_{0}$, none occur in $C_{1}$ (by the assumption in the claim), thus they are $C_{0}$-ubiquitous. In particular, $\operatorname{Symb}\left(C_{0}^{\prime}\right) \subseteq \operatorname{Symb}\left(S_{J}\right)$ for any subclause $S_{J}$ of $C_{0}$, which implies that there exists homomorphisms $C_{0}^{\prime} \rightarrow S_{J} \rightarrow C_{0}$ contradicting the assumption that $C_{0}$ is non-redundant. Thus, $C_{0}^{\prime}$ shares some common symbols with $C_{1}$, and therefore $C_{0}^{\prime}, C_{1}, C_{2}, \ldots, C_{k}$ is also a left-right path of minimal length. Since the previous argument applies to this path as well, symbols in $C_{0}^{\prime}$ are also partitioned into those common with $C_{1}$ and symbols ubiquitous in $C_{0}^{\prime}$. Consider a symbol ubiquitous in $C_{0}^{\prime}$ : it must occur on the path $C_{0}, C_{1}, \ldots, C_{k}$ and, since it doesn't occur in $C_{1}$, it must occur in $C_{0}$ (since the path is of minimal length), implying that it is ubiquitous in $C_{0}$. By symmetry, every ubiquitous symbol in $C_{0}$ is also a ubiquitous symbol in $C_{0}^{\prime}$. This proves the claim.

Finally, we prove Theorem C.12, which follows from the next technical lemma. 
Lemma C.19. Let $Q$ be a forbidden query and let $C_{0}, C_{1}, \ldots, C_{k}$ be a left-right path of minimal length. Then (1) There exists at least one left ubiquitous symbol that does not occur in $C_{1}$; in particular, the query $Q$ has at least one left ubiquitous symbol U. (2) More: no ubiquitous symbol occurs in $C_{1}$. (3) for every left clause $C=\forall y S_{J_{1}}(x, y) \vee \forall y S_{J_{2}}(x, y) \vee \cdots$, every subclause $S_{J_{i}}$ has some common symbol with $C_{1}$. (4) If there are more than one left ubiquitous symbols, then each of them occurs in some middle clause. (5) For any clause $C$ other than $C_{1}$, if $\operatorname{Symb}(C) \subseteq \operatorname{Symb}\left(C_{0}\right) \cup \operatorname{Symb}\left(C_{1}\right)$ then $C$ contains some ubiquitous symbol. (6) If $C$ is a middle clause containing some left ubiquitous symbol $U$, then $\operatorname{Symb}(C) \cap \operatorname{Symb}\left(C_{2}\right)=\emptyset$; equivalently, $\operatorname{Symb}(C) \subseteq \operatorname{Symb}\left(C_{0}\right) \cup \operatorname{Symb}\left(C_{1}\right)$.

Proof. (1) Assuming otherwise, then by Claim 3, every symbol of $C_{0}$ must occur in $C_{1}$, Symb $\left(C_{0}\right) \subseteq \operatorname{Symb}\left(C_{1}\right)$ which implies that there exists a homomorphism $C_{0} \rightarrow C_{1}$. (2) We strengthen the claim by showing that no ubiquitous symbol can occur in $C_{1}$. Let $U$ occur in $C_{1}$. We claim that $Q[U:=0]$ is an unsafe query, contradicting the assumption that $Q$ is final. For that we prove that none of the clauses $C_{0}[U:=0], C_{1}[U:=0], C_{2}, \ldots, C_{k}$ is redundant in $Q[U:=0]$. It is easy to see that there is no homomorphism $C[U:=0] \rightarrow C_{i}[U:=0]$ for $i=0,1$, because both $C_{0}, C_{1}$ contain $U$ and that would imply the existence of a homomorphism $C \rightarrow C_{i}$. Assuming there exists a homomorphism $C[U:=0] \rightarrow C_{i}$ for $i \geq 2$; we must have $i=2$, because the path has minimal length. Let $S$ be a non-ubiquitous symbol in $\operatorname{Symb}\left(C_{0}\right) \cap \operatorname{Symb}\left(C_{1}\right)$, thus $S \notin \operatorname{Symb}\left(C_{2}\right)$ since the path is of minimal length. Consider now the left-right path $C_{0}, C, C_{2}, C_{3}, \ldots, C_{k}$ in $Q$. This is also of minimal length, hence by the definition of a forbidden query, $S$ must occur in $C$, but this contradicts the existence of a homomorphism $C[U:=0] \rightarrow C_{2}$. (3) From Claim 5, we know that the $C_{0}$-ubiquitous symbols are the same as the $C$-ubiquitous symbols. Since $C_{0}, C_{1}, \ldots, C_{k}$ is of minimal length and contains all relational symbols of $Q$, then $\operatorname{Symb}(C) \subseteq \operatorname{Symb}\left(C_{0}\right) \cup \operatorname{Symb}\left(C_{1}\right)$. Now, $\operatorname{suppose} \operatorname{that}$ $S_{J_{i}}$ is a sub-clause of $C$ that does not contain any symbol from $C_{1}$. This means that $S_{J_{i}}$ contains only left ubiquitous symbols. Since the query is minimized, then $S_{J_{i}}$ is the only subclause of $C$. But then, $C \rightarrow C_{0}$ because every subclause of $C_{0}$ contains all ubiquitous symbols (i.e., $\left.\operatorname{Symb}\left(S_{J_{i}}\right)\right)$, which is a contradiction.

(4) Suppose there are at least two left ubiquitous symbols $U_{1}, U_{2}$, and suppose $U_{1}$ does not occur in any middle clause. We claim that $Q\left[U_{1}:=0\right]$ is an unsafe query, contradicting the fact that $Q$ is final. To prove the claim, we show that none of the clauses $C_{0}\left[U_{1}:=0\right], C_{1}, \ldots, C_{k}$ in $Q\left[U_{1}:=0\right]$ is redundant. Assume that there exists a homomorphism $C\left[U_{1}:=0\right] \rightarrow C_{i}$ for $i \geq 1$; then $C$ must contain $U_{1}$ (otherwise there exists a homomorphism $C \rightarrow C_{i}$ ), hence $C$ is a left clause, but in that case it also contains $U_{2}$, hence the homomorphism is not possible. Similarly, if there exists a homomorphism $C\left[U_{1}:=0\right] \rightarrow C_{0}\left[U_{1}:=0\right]$, then there exists a homomorphism $C \rightarrow C_{0}$, because $C_{0}$ contains $U_{1}$ in every subclause. (5) Suppose $\operatorname{Symb}(C) \subseteq \operatorname{Symb}\left(C_{0}\right) \cup \operatorname{Symb}\left(C_{1}\right)$. If $C$ has no ubiquitous symbols, then we claim that $\operatorname{Symb}(C) \subseteq \operatorname{Symb}\left(C_{1}\right)$, but this implies that there exists a homomorphism $C \rightarrow C_{1}$ which is a contradiction. To prove the claim, let $S$ be any symbol in $C$. Since $S$ is not ubiquitous, if $S \in \operatorname{Symb}\left(C_{0}\right)$ then it must also belong to $C_{1}$, proving the claim. (6) Assume otherwise, then $C_{0}, C, C_{2}, \ldots, C_{k}$ is also a left-right path of minimal length, hence by item (2) $C$ cannot contain any ubiquitous symbol, contradiction.

\section{C.4 Computing $Q$ on a Block-database}

Recall that $\Phi_{\Delta}(Q)$ denotes the lineage of $Q$ on the TID $\Delta$. As before, a block $B(u, v)$ is a bipartite TID with two distinguished constants $u, v$. We denote by $U(B), V(B)$ the two partitions of the domain of $B$, that is $\operatorname{Dom}(B)=U(B) \cup V(B)$. By definition, $u \in U(B), v \in V(B)$. We define:

$$
Y_{\alpha \beta}(u, v) \stackrel{\text { def }}{=} \Phi_{B(u, v)}\left(Q_{\alpha \beta}(u, v)\right)
$$

where $Q_{\alpha \beta}$ was introduced in Eq. (63). We prove the following theorem, which is the analogous of Theorem 3.4 for type II queries: Recall that $L(\mathbf{G}), L(\mathbf{H})$ are the supports of the left lattice, and the right lattice of the query, and this includes $\hat{1}$. In this section we want to remove $\hat{1}$, and denote $L_{0}(\mathbf{G}) \stackrel{\text { def }}{=} L(\mathbf{G})-\{\hat{1}\}, L_{0}(\mathbf{H}) \stackrel{\text { def }}{=} L(\mathbf{H})-\{\hat{1}\}$.

Theorem C.20. Let $U, V$ be two disjoint sets, and let $\Delta=\bigcup_{u \in U, v \in V} B(u, v)$ be a disjoint union of blocks (that is, no two blocks share any tuple or any constant, expect for endpoints $u, v)$. Then:

$$
\operatorname{Pr}(Q)=(-1)^{|U|+|V|} \sum_{\substack{\sigma: U \rightarrow L_{0}(\mathbf{G}), \tau: V \rightarrow L_{0}(\mathbf{H})}}\left(\prod_{u \in U} \mu(\sigma(u))\right) \cdot\left(\prod_{v \in V} \mu(\tau(v))\right) \cdot \prod_{u \in U, v \in V} \operatorname{Pr}\left(Y_{\sigma(u) \tau(v)}(u, v)\right)
$$

The sum above has exponentially many terms, namely $2^{\left|L_{0}(\mathbf{G})\right|+\left|L_{0}(\mathbf{H})\right|}$; we will show later how to use this formula.

Proof. The TID $\Delta$ is bipartite, hence its domain is partitioned into $\operatorname{Dom}(\Delta)=\bar{U} \cup \bar{V}$. We have $U \subseteq \bar{U}, V \subseteq \bar{V}$, and the inclusions may be strict since each block $B(u, v)$ may have its own left and right constants, other than $u, v$.

By definition, $\Phi_{\Delta}(Q)=\Phi_{\Delta}\left(Q_{\text {left }}\right) \wedge \Phi_{\Delta}\left(Q_{\text {middle }}\right) \wedge \Phi_{\Delta}\left(Q_{\text {right }}\right)$. Since $Q_{\text {left }}=\forall x\left(\vee_{i=1, m} \forall y G_{i}(x, y)\right)$, its lineage is given by $\Phi_{\Delta}\left(Q_{\text {left }}\right)=$ $\bigwedge_{a \in \bar{U}} \Phi_{\Delta}\left(\bigvee_{i=1, m} \forall y G_{i}(a, y)\right)$. When $a \in \bar{U}-U$, then the lineage $\Phi_{\Delta}\left(\bigvee_{i=1, m} \forall y G_{i}(a, y)\right)$ is equal to the lineage restricted to the unique block $B(u, v)$ that contains $a$, i.e. $\Phi_{\Delta}\left(\bigvee_{i=1, m} \forall y G_{i}(a, y)\right)=\Phi_{B(u, v)}\left(\bigvee_{i=1, m} \forall y G_{i}(a, y)\right)$, because, by construction, $a$ can only be connected to constants in the same block. When $a \in U$, then the lineage may span multiple blocks. Using this observation, and repeating it for $Q_{\text {right }}$, we 
derive the following expressions for the lineages:

$$
\begin{aligned}
& \Phi_{\Delta}\left(Q_{\mathrm{left}}\right)=\bigwedge_{a \in \bar{U}} \Phi_{\Delta}\left(\bigvee_{i} \forall y\left(G_{i}(a, y)\right)\right)=\left(\bigwedge_{u \in U} \Phi_{\Delta}\left(\bigvee_{i} \forall y\left(G_{i}(u, y)\right)\right) \wedge\left(\bigwedge_{a \in \bar{U}-U} \Phi_{\Delta}\left(\bigvee_{i} \forall y\left(G_{i}(a, y)\right)\right)\right)\right. \\
& =\left(\bigwedge_{u \in U} \Phi_{\Delta}\left(\bigvee_{i} \forall y\left(G_{i}(u, y)\right)\right)\right) \wedge\left(\bigwedge_{u \in U, v \in V} \Phi_{B(u, v)}\left(Q_{\text {left }}\right)\right) \\
& \Phi_{\Delta}\left(Q_{\text {right }}\right)=\left(\bigwedge_{v \in V} \Phi_{\Delta}\left(\bigvee_{j} \forall x\left(H_{j}(x, v)\right)\right) \wedge\left(\bigwedge_{u \in U, v \in V} \Phi_{B(u, v)}\left(Q_{\text {right }}\right)\right)\right. \\
& \Phi_{\Delta}\left(Q_{\text {middle }}\right)=\bigvee_{u \in U, v \in V} \Phi_{B(u, v)}\left(Q_{\text {middle }}\right) \\
& \Phi_{\Delta}(Q)=\Phi_{\Delta}\left(Q_{\text {left }}\right) \wedge \Phi_{\Delta}\left(Q_{\text {middle }}\right) \wedge \Phi_{\Delta}\left(Q_{\text {right }}\right) \\
& =\left(\bigwedge _ { u \in U } \Phi _ { \Delta } ( \bigvee _ { i } \forall y ( G _ { i } ( u , y ) \underbrace { \wedge C ( u , y ) } _ { \text { part of } Q } ) ) \wedge \bigwedge _ { u \in U , v \in V } \Phi _ { B ( u , v ) } ( Q ) \wedge \left(\bigwedge_{v \in V} \Phi_{\Delta}\left(\bigvee_{j} \forall x(H_{j}(x, v) \underbrace{\wedge C(x, v)}_{\text {part of } Q})\right)\right.\right.
\end{aligned}
$$

In the last line we added the redundant terms $C(u, y)$ and $C(x, v)$ (recall that $Q_{\text {middle }}=\forall x \forall y C(x, y)$ ). Now we will apply Möbius' inversion formula on the expression for $\Phi_{\Delta}(Q)$ above, repeatedly, once for each $u \in U$, then once for each $v \in V$. We show how to do it for one fixed constant $u_{1} \in U$. First, we separate $u_{1}$ from the conjunction $\bigwedge_{u \in U}$ above:

$$
\begin{aligned}
\Phi_{\Delta}(Q) & =\Phi_{\Delta}\left(\bigvee_{i} \forall y\left(G_{i}\left(u_{1}, y\right) \wedge C\left(u_{1}, y\right)\right)\right) \\
& \wedge \underbrace{\operatorname{REST}}_{\left.\bigwedge_{u \in U-\left\{u_{1}\right\}} \Phi_{\Delta}\left(\bigvee_{i} \forall y\left(G_{i}(u, y) \wedge C(u, y)\right)\right) \wedge \bigwedge_{u \in U, v \in V} \Phi_{B(u, v)}(Q) \wedge \bigwedge_{v \in V} \Phi_{\Delta}\left(\bigvee_{j} \forall x\left(H_{j}(x, v) \wedge C(x, v)\right)\right)\right)} \\
& =\Phi_{\Delta}\left(\bigvee_{i} \forall y\left(G_{i}\left(u_{1}, y\right) \wedge C\left(u_{1}, y\right)\right)\right) \wedge \operatorname{REST}=\bigvee_{i}\left(\Phi_{\Delta}\left(\forall y G_{i}\left(u_{1}, y\right) \wedge C\left(u_{1}, y\right)\right) \wedge \text { REST }\right) \\
\operatorname{Pr}(Q)=\operatorname{Pr}\left(\Phi_{\Delta}(Q)\right) & =-\sum_{\alpha_{1} \in L_{0}(\mathrm{G})} \mu\left(\alpha_{1}\right) \operatorname{Pr}\left(\Phi_{\Delta}\left(\forall y\left(G_{\alpha_{1}}\left(u_{1}, y\right) \wedge C\left(u_{1}, y\right)\right)\right) \wedge \operatorname{REST}\right)
\end{aligned}
$$

Recall that the lattice $\hat{L}_{0}(\mathrm{G})$ was defined by the formulas $G_{1} \wedge C, G_{2} \wedge C, \ldots$, and not by $G_{1}, G_{2}, \ldots$ We introduced earlier the term $C\left(u_{1}, y\right)$ in order to be able to apply the Möbius formula for this lattice. Next, we consider formula REST, which contains the conjunction $\bigwedge_{u \in U-\left\{u_{1}\right\}}$. We separate a second $u_{2} \in U-\left\{u_{1}\right\}$, and repeat this argument for $u_{2}, u_{3}, \ldots \in U$, reducing the REST formula, until we arrive at:

$$
\begin{array}{r}
\operatorname{Pr}(Q)=(-1)^{|U|} \sum_{\sigma: U \rightarrow L_{0}(\mathrm{G})}\left(\prod_{u \in U} \mu(\sigma(u))\right) \operatorname{Pr}\left(\bigwedge_{u \in U} \Phi_{\Delta}\left(\forall y\left(G_{\sigma(u)}(u, y) \wedge C(u, y)\right)\right) \wedge \operatorname{REST}\right) \\
\text { where REST } \stackrel{\text { def }}{=} \bigwedge_{u \in U, v \in V} \Phi_{B(u, v)}(Q) \wedge\left(\bigwedge_{v \in V} \Phi_{\Delta}\left(\bigvee_{j} \forall x\left(H_{j}(x, v) \wedge C(x, v)\right)\right)\right)
\end{array}
$$

We repeat the same process on the right clauses $\bigwedge_{v \in V} \Phi_{\Delta}\left(\vee_{j} \forall x\left(H_{j}(x, v) \wedge C(x, v)\right)\right)$, and obtain:

$$
\begin{aligned}
\operatorname{Pr}(Q)= & (-1)^{|U|+|V|} \sum_{\sigma: U \rightarrow L_{0}(\mathbf{G}), \tau: V \rightarrow L_{0}(\mathbf{H})}\left(\prod_{u \in U} \mu(\sigma(u))\right)\left(\prod_{v \in V} \mu(\tau(v))\right) \\
& \operatorname{Pr}\left(\bigwedge_{u \in U} \Phi_{\Delta}\left(\forall y\left(G_{\sigma(u)}(u, y) \wedge C(u, y)\right)\right) \wedge \bigwedge_{u \in U, v \in V} \Phi_{B(u, v)}(Q) \wedge \bigwedge_{v \in V} \Phi_{\Delta}\left(\forall x\left(H_{\tau(v)}(x, v) \wedge C(x, v)\right)\right)\right)
\end{aligned}
$$


Next, we observe that:

$$
\begin{gathered}
\Phi_{\Delta}\left(\forall y\left(G_{\sigma(u)}(u, y) \wedge C(u, y)\right)\right)=\bigwedge_{v \in V} \Phi_{B(u, v)}\left(\forall y\left(G_{\sigma(u)}(u, y) \wedge C(u, y)\right)\right) \\
\Phi_{\Delta}\left(\forall x\left(H_{\tau(v)}(x, v) \wedge C(x, v)\right)\right)=\bigwedge_{u \in U} \Phi_{B(u, v)}\left(\forall x\left(H_{\tau(v)}(x, v) \wedge C(x, v)\right)\right)
\end{gathered}
$$

Since $C$ is the middle part of $Q$, we have $\Phi_{B(u, v)}(C(u, y) \wedge Q) \equiv \Phi_{B(u, v)}(Q)$ and therefore we can eliminate $C(u, v)$ and obtain:

$$
\begin{aligned}
\operatorname{Pr}(Q) & =(-1)^{|U|+|V|} \sum_{\sigma: U \rightarrow L_{0}(\mathbf{G}), \tau: V \rightarrow L_{0}(\mathbf{H})}\left(\prod_{u \in U} \mu(\sigma(u))\right)\left(\prod_{v \in V} \mu(\tau(v))\right) \operatorname{Pr}\left(\bigwedge_{u \in U, v \in V} \Phi_{B(u, v)}\left(\forall y\left(G_{\sigma(u)}(u, y)\right)\right) \wedge \Phi_{B(u, v)}(Q) \wedge \Phi_{B(u, v)}\left(\forall x\left(H_{\tau(v)}(x, v)\right)\right)\right) \\
& =(-1)^{|U|+|V|} \sum_{\sigma: U \rightarrow L_{0}(\mathbf{G}), \tau: V \rightarrow L_{0}(\mathbf{H})}\left(\prod_{u \in U} \mu(\sigma(u))\right)\left(\prod_{v \in V} \mu(\tau(v))\right) \operatorname{Pr}\left(\bigwedge_{u \in U, v \in V} \Phi_{B(u, v)}\left(\forall y\left(G_{\sigma(u)}(u, y)\right) \wedge Q \wedge \forall x\left(H_{\tau(v)}(x, v)\right)\right)\right)(72) \\
& =(-1)^{|U|+|V|} \sum_{\sigma: U \rightarrow L_{0}(\mathbf{G}), \tau: V \rightarrow L_{0}(\mathbf{H})}\left(\prod_{u \in U} \mu(\sigma(u))\right)\left(\prod_{v \in V} \mu(\tau(v))\right) \prod_{u \in U, v \in V} \operatorname{Pr}\left(\Phi_{B(u, v)}\left(\forall y\left(G_{\sigma(u)}(u, y)\right) \wedge Q \wedge \forall x\left(H_{\tau(v)}(x, v)\right)\right)\right)
\end{aligned}
$$

This completes the proof of Theorem C.20.

Let $G(U, V, E)$ be a bipartite graph, defining a CCP problem. As for Type I queries (Eq. (4)) we construct a TID that is a union of disjoint blocks $\bigcup_{u \in U, v \in V} B(u, v)$, where for every non-edge $(a, b) \notin E$ we define $B(a, b)$ to be the trivial block where all tuples have probability 1 . However, unlike the formula (4) for type I queries, now we need to cope the products of Möbius functions in Eq. (67). To remove those, we extend the graph by adding, for each node $u \in U$, one outgoing edge $\left(u, u^{\prime}\right)$ where $u^{\prime}$ is a fresh node with no other incoming edges; similarly, we add edges $\left(v^{\prime}, v\right)$, one for each node $v \in V$. More precisely, the new graph is $\left(U \cup V^{\prime}, V \cup U^{\prime}, E \cup E^{\prime}\right)$, where $V^{\prime}=\left\{v^{\prime} \mid v \in V\right\}$, $U^{\prime}=\left\{u^{\prime} \mid u \in U\right\}$ and $E^{\prime}=\left\{\left(u, u^{\prime}\right) \mid u \in U\right\} \cup\left\{\left(v, v^{\prime}\right) \mid v \in V\right\}$. Then, we have:

Corollary C.21. Given the notations above:

$$
\begin{aligned}
\operatorname{Pr}(Q)= & \sum_{\substack{\sigma: U \rightarrow L_{0}(\mathbf{G}), \tau: V \rightarrow L_{0}(\mathbf{H})}}\left(\prod_{u \in U} \mu(\sigma(u))\right) \cdot\left(\prod_{v \in V} \mu(\tau(v))\right) \cdot \prod_{(u, v) \in E} \operatorname{Pr}\left(Y_{\sigma(u) \tau(v)}(u, v)\right) \cdot \prod_{u \in U} \operatorname{Pr}\left(Y_{\sigma(u), \hat{1}}\left(u, u^{\prime}\right)\right) \cdot \prod_{v \in V} \operatorname{Pr}\left(Y_{\hat{1} \tau(v)}\left(v^{\prime}, v\right)\right) \\
\text { where } Y_{\alpha \beta}(u, v) & \stackrel{\text { def }}{=} \Phi_{B(u, v)}\left(G_{\alpha}(u) \wedge Q \wedge H_{\alpha}(v)\right) \\
Y_{\alpha \hat{1}}\left(u, u^{\prime}\right) & \stackrel{\text { def }}{=} \Phi_{B\left(u, u^{\prime}\right)}\left(G_{\alpha}(u) \wedge Q\right)=\Phi_{B\left(u, u^{\prime}\right)}\left(G_{\alpha}(u) \wedge Q \wedge H_{\hat{1}}\left(u^{\prime}\right)\right) \quad \text { see Eq. }(65) \\
Y_{\hat{1} \beta}\left(v^{\prime}, v\right) & \stackrel{\text { def }}{=} \Phi_{B\left(v^{\prime}, v\right)}\left(Q \wedge H_{\alpha}(v)\right)=\Phi_{B\left(v^{\prime}, v\right)}\left(G_{\hat{1}}\left(v^{\prime}\right) \wedge Q \wedge H_{\alpha}(v)\right)
\end{aligned}
$$

Proof. (Sketch) The proof consists of a straightforward extension of the proof of Theorem C.20. We begin the proof similarly, but in Eq.(68) we replace the sets $U$ and $V$ by $U \cup V^{\prime}$ and $V \cup U^{\prime}$ respectively. Next, we apply the Möbius inversion formula repeatedly, once to each $u \in U$ as before, but we do not apply it to any $v^{\prime} \in V^{\prime}$. Therefore, there are two changes to the expression REST in (69). First, it will have the following residual:

$$
\bigwedge_{v^{\prime} \in V^{\prime}} \Phi_{\Delta}\left(\bigvee_{i} \forall y G_{i}\left(v^{\prime}, y\right)\right)
$$

Since $v^{\prime}$ has a single outgoing edge $\left(v^{\prime}, v\right)$, this residual is restricted to the lineage in the block $B\left(v^{\prime}, v\right)$, i.e. it is equivalent to:

$$
\bigwedge_{v \in V} \Phi_{B\left(v^{\prime}, v\right)}\left(\bigvee_{i} \forall y G_{i}\left(v^{\prime}, y\right)\right)
$$

The second change in (69) is that we need to expand the expressions $\bigwedge_{u \in U, v \in V} \Phi_{B(u, v)}(Q)$ with $\bigwedge_{u \in U} \Phi_{B\left(u, u^{\prime}\right)}(Q)$ and $\bigwedge_{v \in V} \Phi_{B\left(v^{\prime}, v\right)}(Q)$. The latter absorbs the residual (73), because $\left(\bigvee_{i} \forall y G_{i}(x, y)\right)$ is implied by $Q$ (i.e. it is one of the conjuncts of $\left.Q\right)$ :

$$
\forall v \in V: \quad \quad \Phi_{B\left(v^{\prime}, v\right)}\left(\bigvee_{i} \forall y G_{i}\left(v^{\prime}, y\right)\right) \wedge \Phi_{B\left(v^{\prime}, v\right)}(Q) \equiv \Phi_{B\left(v^{\prime}, v\right)}(Q)
$$

Similarly, when we apply Möbius inversion formula to each $v \in V$ we do not apply it to any $u^{\prime} \in U^{\prime}$, and are left with a similar residual on the right, which also gets absorbed. Thus, the only change to Eq. (70) is the addition of the boolean formulas $\bigwedge_{u \in U} \Phi_{B\left(u, u^{\prime}\right)}(Q)$ and $\bigwedge_{v \in V} \Phi_{B\left(v^{\prime}, v\right)}(Q)$. Next, we need to modify Eq. (71) from $\bigwedge_{v \in V}$ to $\bigwedge_{v \in\left(V \cup\left\{u^{\prime}\right\}\right)}$, in effect adding the conjunct $\bigwedge_{u \in U} \Phi_{B\left(u, u^{\prime}\right)}\left(\forall y G_{\sigma(u)}(u, y)\right)$, and similarly for the line below Eq. (71). The Boolean formula under $\operatorname{Pr}(\cdots)$ in Eq. (72) becomes:

$$
\bigwedge_{u \in U, v \in V} \Phi_{B(u, v)}\left(\forall y\left(G_{\sigma(u)}(u, y)\right) \wedge Q \wedge \forall x\left(H_{\tau(v)}(x, v)\right)\right) \wedge \bigwedge_{u \in U} \Phi_{B\left(u, u^{\prime}\right)}\left(\forall y\left(G_{\sigma(u)}(u, y)\right) \wedge Q\right) \wedge \bigwedge_{v \in V} \Phi_{B\left(v^{\prime}, v\right)}\left(\wedge Q \wedge \forall x\left(H_{\tau(v)}(x, v)\right)\right)
$$


Since distinct blocks do not share any tuples, the conjuncts above are independent, hence, as before, the probability is their $\operatorname{product}, \operatorname{Pr}(Q)=$

$$
\prod_{u \in U, v \in V} \operatorname{Pr}\left(\Phi_{B(u, v)}\left(\forall y\left(G_{\sigma(u)}(u, y)\right) \wedge Q \wedge \forall x\left(H_{\tau(v)}(x, v)\right)\right)\right) \cdot \prod_{u \in U} \operatorname{Pr}\left(\Phi_{B\left(u, u^{\prime}\right)}\left(\forall y\left(G_{\sigma(u)}(u, y)\right) \wedge Q\right)\right) \cdot \prod_{v \in V} \operatorname{Pr}\left(\Phi_{B\left(v^{\prime}, v\right)}\left(\wedge Q \wedge \forall x\left(H_{\tau(v)}(x, v)\right)\right)\right)
$$

Finally, we notice that $u \in U, v \in V$ in the first product can be replaced by $(u, v) \in E$, because all non-edges are trivially true.

We can now describe the reduction from $\operatorname{CCP}$ to $\operatorname{GFOMC}(Q)$. Let $Q$ be any bipartite, unsafe type II query (meaning: type II-II), and let $\bar{m}, \bar{n}$ be the sizes of its lattice supports $L_{0}(\mathrm{G})$ and $L_{0}(\mathbf{H})$. Since $Q$ is unsafe, we have $\bar{m}, \bar{n} \geq 3$. Our goal is to prove $\operatorname{CCP}(\bar{m}, \bar{n}) \leq P$ GFOMC $(Q)$. Fix an instance of a $\operatorname{CCP}(\bar{m}, \bar{n})$ problem $(U, V, E)$. We extend the graph as before to $\left(U \cup V^{\prime}, V \cup U^{\prime}, E \cup E^{\prime}\right)$. We define the TID $\Delta$ to be a union of blocks $B(u, v)$ for all $(u, v) \in E \cup E^{\prime}$; as before, $B(a, b)$ is trivially true when $(a, b)$ is not an edge. The blocks (to be defined in the next section) will be isomorphic, and therefore, the following quantities do not depend on $u, v$ :

$$
\begin{array}{ll}
y_{\alpha \beta} \stackrel{\text { def }}{=} \operatorname{Pr}\left(Y_{\alpha, \beta}(u, v)\right) & u \in U, v \in V \\
y_{\alpha *} \stackrel{\text { def }}{=} \operatorname{Pr}\left(Y_{\alpha, \hat{1}}\left(u, u^{\prime}\right)\right) & u \in U \\
y_{\hat{1} \beta} \stackrel{\text { def }}{=} \operatorname{Pr}\left(Y_{\hat{1} \beta}\left(v^{\prime}, v\right)\right) & v \in V
\end{array}
$$

By Corollary C.21, we obtain:

$$
\operatorname{Pr}(Q)=\sum_{\substack{\sigma: U \rightarrow L_{0}(\mathbf{G}) \\ \tau: V \rightarrow L_{0}(\mathbf{H})}}\left(\prod_{u \in U} \mu(\sigma(u))\right) \cdot\left(\prod_{v \in V} \mu(\tau(v))\right) \cdot\left(\prod_{(u, v) \in E} y_{\sigma(u), \tau(v)}\right) \cdot\left(\prod_{u \in U} y_{\sigma(u), \hat{1}}\right) \cdot\left(\prod_{v \in V} y_{\hat{1} \tau(v)}\right)
$$

Recall that $L_{0}(\mathrm{G})=L(\mathrm{G})-\{\hat{1}\}$ is the strict support of the left lattice, and $\bar{m}$ is its size. Consider now the coloring counting problem given by the the graph $(U, V, E)$. Every pair $\sigma, \tau$ defines a coloring of the graph $(U, V, E)$. Denoting $\mathbf{k} \stackrel{\text { def }}{=}\left\{k_{\alpha, \beta}, k_{\alpha, \hat{1}}, k_{\hat{1}, \beta} \mid \alpha \subseteq[\bar{m}], \beta \subseteq[\bar{n}]\right\}$ its signature, the factor $y_{\alpha \beta}$ will occur precisely $k_{\alpha \beta}$ times, i.e. with the exponent $k_{\alpha \beta}$ where $k_{\alpha \beta}$ is the number of edges in the bipartite graph whose edges are colored $\alpha$ and $\beta$ respectively. The factor $y_{\hat{1}, \beta}$ will occur $k_{\hat{1}, \beta}$ times where $k_{\hat{1}, \beta}$ represents the number of nodes in $V$ colored $\beta$. Likewise, $k_{\alpha, \hat{1}}$ represents the number of nodes in $U$ colored $\alpha$. Therefore:

$$
\operatorname{Pr}(Q)=\sum_{\mathbf{k}} \# \mathbf{k}\left(\prod_{\alpha}(\mu(\alpha))^{k_{\alpha, \hat{1}}}\right) \cdot\left(\prod_{\beta}(\mu(\beta))^{k_{\hat{1}, \beta}}\right) \cdot\left(\prod_{\alpha, \beta} y_{\alpha \beta}^{k_{\alpha \beta}}\right) \cdot\left(\prod_{\beta} y_{\hat{1}, \beta}^{k_{\hat{1}, \beta}}\right) \cdot\left(\prod_{\alpha} y_{\alpha, \hat{1}}^{k_{\alpha, \hat{1}}}\right)
$$

where $\alpha, \beta$ range over the strict supports $L_{0}(\mathrm{G}), L_{0}(\mathrm{H})$ (i.e. are $\left.\neq \hat{1}\right)$. Our unknowns are \#k; there is one unknown for every coloring signature $\mathbf{k}$. We introduce new variables $x_{\mathbf{k}}$, indexed by the signatures $\mathbf{k}$, as follows:

$$
x_{\mathbf{k}} \stackrel{\text { def }}{=} \# \mathbf{k} \cdot\left(\prod_{\alpha}(\mu(\alpha))^{k_{\alpha, \hat{1}}}\right) \cdot\left(\prod_{\beta}(\mu(\beta))^{k_{\hat{1}, \beta}}\right)
$$

Thus, one call to the oracle for $\operatorname{Pr}(Q)$ computes the following linear combination of the unknowns $x_{\mathbf{k}}$ :

$$
\operatorname{Pr}(Q)=\sum_{\mathbf{k}}\left(\prod_{\substack{\alpha \in L(\mathbf{G}) \\ \beta \in L(\mathbf{H}) \\(\alpha, \beta) \neq(\hat{1}, \hat{1})}} y_{\alpha \beta}^{k_{\alpha \beta}}\right) x_{\mathbf{k}}
$$

Notice that here $\alpha, \beta$ range over the entire support, i.e. including $\hat{1}$, except of the combination $\alpha=\hat{1}, \beta=\hat{1}$, because that does not occur in $\operatorname{Pr}(Q)$, Eq. (75).

Let $h \stackrel{\text { def }}{=}(\bar{m}+1)(\bar{n}+1)=O(1)$. The equation above has $(M+1)^{h}$ unknowns $x_{\mathbf{k}}$ because for every pair $\alpha \in L(\mathbf{G})$ and $\beta \in L(\mathbf{H})$ there can be between 0 and $M$ blocks with the $\alpha \beta$ configuration. Accordingly, there are $(M+1)^{h}$ coefficients $y_{\alpha \beta}^{k_{\alpha \beta}}$. To simplify the notation, lets denote the pair $\alpha \beta$ by a single index $i$, where $i=1, \ldots, h$. The equation becomes:

$$
\operatorname{Pr}(Q)=\sum_{k_{1}, \ldots, k_{h} \in\{0, \ldots, M\}}\left(\prod_{i=1, h} y_{i}^{k_{i}}\right) x_{k_{1} k_{2} \cdots k_{h}}
$$


Let $\mathbf{p}=\left\{p_{1}, \ldots, p_{h}\right\}$ be a set of $h$ natural numbers where $p_{i} \geq 1$. We will show in the next several sections how to construct a block $B(u, v)$ that depends on $\mathbf{p}$, hence we denote it $B^{(\mathbf{p})}(u, v)$, where all tuples have probabilities in $\{0,1 / 2,1\}$, such that:

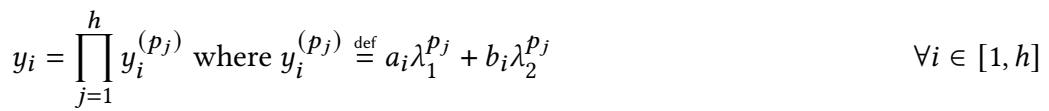

where $\lambda_{1}, \lambda_{2}$ and $a_{i}, b_{i}, i \in[1, h]$ are constants independent of $p_{1}, \ldots, p_{h}$ satisfying the following.

$$
\begin{array}{rrr}
\lambda_{1} \neq \pm \lambda_{2} & \text { and } & \lambda_{1} \neq 0, \lambda_{2} \neq 0 \\
b_{i} \neq 0 & \forall i \in[h] \\
a_{i} b_{j} \neq a_{j} b_{i} & i \neq j
\end{array}
$$

By Theorem 3.7, if we set the values of $p_{1}, \ldots, p_{h}$ independently to $1,2, \ldots,(M+1)$, then we obtain a system with $(M+1)^{h}$ equations whose matrix is non-singuar, from which we can compute the unknowns \#k in polynomial time (by Gaussian elimination), and thus sholve the instance of the $\operatorname{CCP}(\bar{m}, \bar{n})$ problem. In the next section we describe how to construct the block $B \mathbf{P}(u, v)$ to ensure that the probability $(77)$ is given by an expression of the type (77). We notice that $B^{\mathbf{P}}(u, v)$ has the same structure for all $u$, $v$; in what follows we only discuss a single block $B \mathbf{P}(u, v)$.

\section{C.5 Designing the Block $B^{(\mathbf{p})}(u, v)$}

Fix $\mathbf{p}=\left(p_{1}, \ldots, p_{h}\right)$ a vector of $h$ natural numbers $\geq 1$. We describe here the block $B^{(\mathbf{p})}(u, v)$; its tuples and probabilities are the same for all choices of $u, v$, thus our discussion below does not depend on $u$, v. The block $B^{(\mathbf{p})}(u, v)$ will consists of a union of $h$ blocks, each corresponding to one of the parameters $p_{1}, \ldots, p_{h}$ :

$$
B^{(\mathbf{p})}(u, v)=\bigcup_{j=1, h} B_{j}^{p_{j}}(u, v)
$$

Since the blocks $B_{1}^{p_{1}}, \ldots, B_{h}^{p_{h}}$ have disjoint sets of tuples, we have:

$$
\forall \alpha \in L(\mathrm{G}), \beta \in L(\mathbf{H}): \quad Y_{\alpha \beta}^{(\mathbf{p})}(u, v) \stackrel{\text { def }}{=} \Phi_{B^{(\mathbf{p})}(u, v)}\left(G_{\alpha}(u) \wedge Q \wedge H_{\beta}(v)\right)=\bigwedge_{j=1, h} \Phi_{B_{j}^{p_{j}}(u, v)}\left(G_{\alpha}(u) \wedge Q \wedge H_{\beta}(v)\right) \stackrel{\text { def }}{=} \bigwedge_{j=1, h} Y_{\alpha \beta, j}^{\left(p_{j}\right)}
$$

Indeed, each clause of the lineage of $G_{\alpha}(u)$ lies entirely within one block $B_{j}^{p_{j}}$, because $G_{\alpha}(u)=\forall y G_{\alpha}(u, y)$ has a single variable $\forall y$ (see Eq. (61)). Consider now the query $Q$. The only clauses whose lineage may span multiple blocks are those in $Q_{\text {left }} \equiv \forall x\left(\vee_{j=1, m} \forall y G_{j}(x, y)\right)$, and only those clauses obtained by mapping $x$ to $u$. But that formula is absorbed by $G_{\alpha}(u)$, in other words:

$$
\left(\forall y G_{\alpha}(u, y)\right) \wedge\left(\bigvee_{j=1, m} \forall y G_{j}(u, y)\right) \equiv \forall y G_{\alpha}(u, y)
$$

Therefore, the probability $y_{\alpha \beta}=\operatorname{Pr}\left(Y_{\alpha \beta}(u, v)\right)$ is a product of probabilities one for each block:

$$
\forall \alpha \in L(\mathbf{G}), \beta \in L(\mathbf{H}): \quad y_{\alpha \beta}^{(\mathbf{p})}=\prod_{j=1, h} \operatorname{Pr}\left(\Phi_{B_{j}^{\left(p_{j}\right)}(u, v)}\left(G_{\alpha}(u) \wedge Q \wedge H_{\beta}(v)\right)\right) \stackrel{\text { def }}{=} \prod_{j=1, h} y_{\alpha \beta, j}^{\left(p_{j}\right)}
$$

We will design the blocks $B_{j}^{\left(p_{j}\right)}(u, v)$ to be similar, and differ only in their parameter $p_{j}$. To simplify the notation, we drop the index $j$ : thus, the expressions $p_{j}, B_{j}^{\left(p_{j}\right)}(u, v), y_{\alpha \beta, j}, Y_{\alpha \beta, j}^{\left(p_{j}\right)}$, etc become $p, B^{(p)}(u, v), y_{\alpha \beta}, Y_{\alpha \beta}^{(p)}$. Our goal is to design the block $B^{(p)}(u, v)$, where $p \geq 1$ is a natural number, such that, for all $\alpha, \beta$ (including $\hat{1}$ ), we have:

$$
y_{\alpha \beta}^{(p)}=\operatorname{Pr}\left(Y_{\alpha \beta}\right)=a_{\alpha \beta} \lambda_{1}^{p}+b_{\alpha \beta} \lambda_{2}^{p}
$$

where the parameters $\lambda_{1}, \lambda_{2}, a_{\alpha \beta}, a_{\alpha, \hat{1}}$, etc, are independent of $p$ and satisfy the conditions (78)-(80).

We describe now the block $B^{(p)}(u, v)$, and will refer to Fig. 3. Let $m$ be the maximum number of subclauses in any left or right clause; notice that $m \geq 2$. An elementary block $B(a, b)$ is the set of tuples $B(a, b) \stackrel{\text { def }}{=}\left\{S_{1}(a, b), S_{2}(a, b), \ldots\right\}$, i.e. there is exactly one tuple $S(a, b)$ for each binary symbol $S \in \mathcal{R}$.

Definition C.22. The block $B^{(p)}(u, v)$ is the disjoint union of the following blocks:

- A prefix block $B_{\text {pref }}\left(u, r_{0}\right)$, which, in turn, is the disjoint union of $r$ parallel blocks:

$$
B_{\text {pref }}\left(u, r_{0}\right)=\bigcup_{i=1, r}\left(B\left(u, t_{\text {pref }, i}\right) \cup B\left(r_{0}, t_{\text {pref }, i}\right)\right)
$$

where $B\left(u, t_{\text {pref }, i}\right), B\left(r_{0}, t_{\text {pref, } i}\right)$ are elementary blocks. The number $r$ will be chosen later. 
- A zig-zag part, which is a union of $2 p+1$ elementary blocks:

$$
B\left(r_{0}, t_{0}\right) \cup B\left(r_{1}, t_{0}\right) \cup B\left(r_{1}, t_{1}\right) \cup \ldots \cup B\left(r_{p}, t_{p-1}\right) \cup B\left(r_{p}, t_{p}\right)
$$

- A suffix block $B_{\text {suff }}\left(t_{p}, v\right)$, which is the union of $r$ parallel blocks (same $r$ as for the prefix):

$$
B_{\text {suff }}\left(t_{p}, v\right)=\bigcup_{i=1, r}\left(B\left(r_{\text {suff }, i}, t_{p}\right) \cup B\left(r_{\text {suff }, i}, v\right)\right)
$$

where $B\left(r_{\text {suff }, i}, t_{p}\right), B\left(r_{\text {suff }, i}, v\right)$ are elementary blocks.

- For each constant $r_{i}$ (including $r_{i, \text { suff }}$ ) introduced above there are $m-2$ dead-end branches of elementary blocks: $B\left(r_{i}, e_{i}^{(1)}\right) \cup \ldots \cup$ $B\left(r_{i}, e_{i}^{(m-2)}\right)$.

- For each constant $t_{i}$ introduced above (including $\left.t_{\text {pref, } i}\right)$ there are $m-2$ dead-end branches of elementary blocks: $B\left(f_{i}^{(1)}, t_{i}\right) \cup \ldots \cup$ $B\left(f_{i}^{(m-2)}, t_{i}\right)$.

- For any other pairs of constants $a, b$ not explicitly mentioned above, there is a trivial elementary block $B(a, b)$ where all tuples have probability 1 .

We denote by $B\left(r_{0}, t_{p}\right)$ the $z i g$-zag portion of the block:

$$
B\left(r_{0}, t_{p}\right)=B\left(r_{0}, t_{0}\right) \cup \bigcup_{i=1, p}\left(B\left(r_{i}, t_{i-1}\right) \cup B\left(r_{i}, t_{i}\right) \cup \bigcup_{j}^{(\underbrace{\left(B\left(r_{i}, e_{i}^{(j)}\right) \cup B\left(f_{i-1}^{(j)}, t_{i-1}\right)\right)}_{\text {dead ends }})}\right.
$$

Therefore the entire block is:

$$
B^{(p)}(u, v)=B_{\text {pref }}\left(u, r_{0}\right) \cup(\underbrace{\bigcup_{j} B\left(r_{0}, e_{0}^{(j)}\right)}_{\text {dead end }}) \cup B\left(r_{0}, t_{p}\right) \cup(\underbrace{\left.\bigcup_{j}^{\bigcup_{\text {dea }} B\left(f_{i}^{(j)}, t_{p}\right)}\right)}_{\text {dead end }}
$$

\section{C.6 Two Properties of $Y_{\alpha \beta}^{(p)}$}

In this section we prove two properties of $Y_{\alpha \beta}^{(p)}$. First, the mapping $(\alpha, \beta) \mapsto Y_{\alpha \beta}^{(p)}$ is invertible (see Lemma C.16). Second, if $Q$ is a forbidden query, then $Y_{\alpha \beta}^{(p)}$ is a connected Boolean formula, for every $\alpha \in L(\mathrm{G}), \beta \in L(\mathbf{H})$ (see Lemma C.15). To simplify the discussion, we consider $Y_{\alpha \beta}^{(p)}$ to be the lineage on the zig-zag block $B^{(p)}\left(r_{0}, t_{p}\right)(82)$, and will not consider the prefix and the suffix blocks of $B^{(p)}(u, v)$; the proofs of both properties extend immediately to the complete block $B^{(p)}(u, v)$. Thus, $Y_{\alpha \beta}^{(p)}$ means the the lineage restricted to the zig-zag block $B^{(p)}\left(r_{0}, t_{p}\right)$ :

$$
Y_{\alpha \beta}^{(p)} \stackrel{\text { def }}{=} \Phi_{B\left(r_{0}, t_{p}\right)}\left(G_{\alpha}\left(r_{0}\right) \wedge Q \wedge H_{\beta}\left(t_{p}\right)\right) \quad y_{\alpha \beta}^{(p)} \stackrel{\text { def }}{=} \operatorname{Pr}\left(Y_{\alpha \beta}^{(p)}\right)
$$

As usual, each tuple in $B^{(p)}\left(r_{0}, t_{p}\right)$ becomes a Boolean variable.

Lemma C.23. The mapping $(\alpha, \beta) \mapsto Y_{\alpha \beta}^{(p)}$ is invertible. More precisely, if the logical implication $\forall x \forall y Y_{\alpha_{1} \beta_{1}}^{(p)}(x, y) \Rightarrow \forall x \forall y Y_{\alpha_{2} \beta_{2}}^{(p)}(x, y)$ holds, then $\alpha_{1} \leq \alpha_{2}$ and $\beta_{1} \leq \beta_{2}$, in the lattices $\hat{L}(\mathbf{G})$ and $\hat{L}(\mathbf{H})$ respectively.

Proof. The proof is identical to that of Lemma C.16 and omitted.

LEMmA C.24. Let $Q$ be a forbidden query of type II. Then, for all $\alpha \in L(\mathrm{G}), \beta \in L(\mathbf{H})$, the Boolean formulas $Y_{\alpha \beta}^{(p)}$ are connected, and depend on all Boolean variables in the block $B^{(p)}\left(r_{0}, t_{0}\right)$.

Proof. The proof extends the proof idea in lemma C.15, and it is slightly simpler because $Q$ is forbidden. Let $C_{0}, C_{1}, \ldots, C_{k}$ be a leftto-right path in $Q$, of minimal length; we will use it to construct a long path of clauses in $Y_{\alpha \beta}^{(p)}$ that goes through all zig-zag blocks. We start with one block in the zig-zag fragment, say $B\left(r_{i}, t_{i-1}\right)$. The middle clauses $C_{1}, \ldots, C_{k-1}$ have only two logical variables $x, y$, e.g. $\forall x \forall y\left(S_{1}(x, y) \vee S_{2}(x, y) \vee \cdots\right)$, hence their groundings $C_{1}\left(r_{i}, t_{i-1}\right), C_{2}\left(r_{i}, t_{i-1}\right), \ldots C_{p-1}\left(r_{i}, t_{i-1}\right)$ are non-redundant clauses (since they were non-redundant in $Q)$ and are connected. Similarly, on the next block in the zig-zag, $C_{1}\left(r_{i}, t_{i}\right), C_{2}\left(r_{i}, t_{i}\right), \ldots C_{k-1}\left(r_{i}, t_{i}\right)$ are non-redundant and connected. It remains to connect $C_{1}\left(r_{i}, t_{i-1}\right)$ and $C_{1}\left(r_{i}, t_{i}\right)$ and for that we use the left clause $C_{0}=\forall x \bigvee_{k=1, \ell} \forall y S_{J_{k}}(x, y)$. Consider the first 


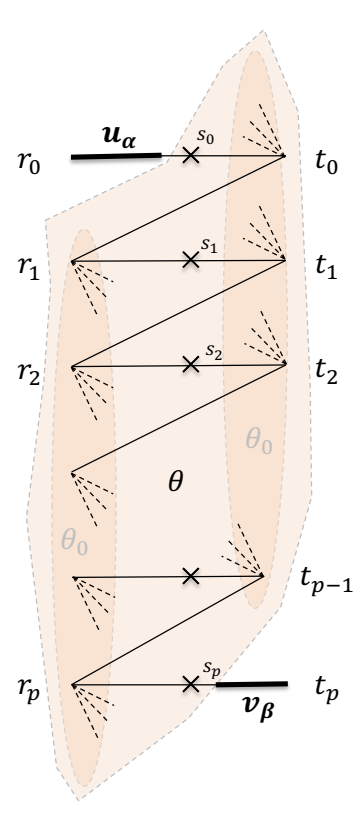

(a)

Choose $\theta_{0} \subseteq \theta$ such that:

$0<\left|\lambda_{1}\right|<\lambda_{2}$

$\forall \alpha, \beta: b_{\alpha \beta}>0$

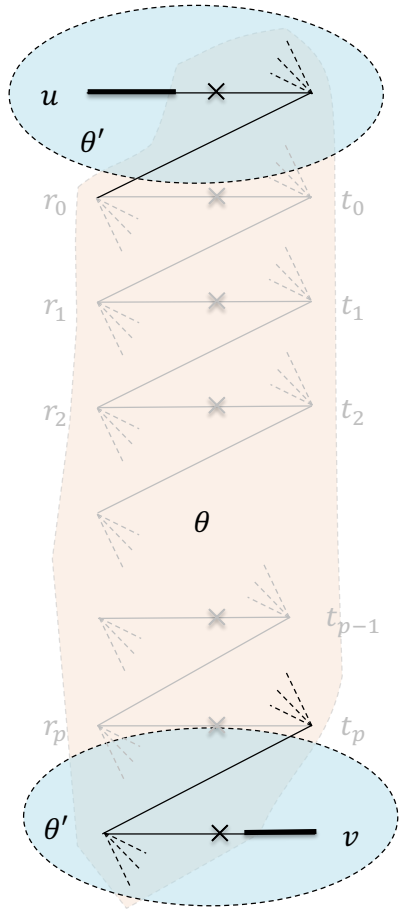

(b)

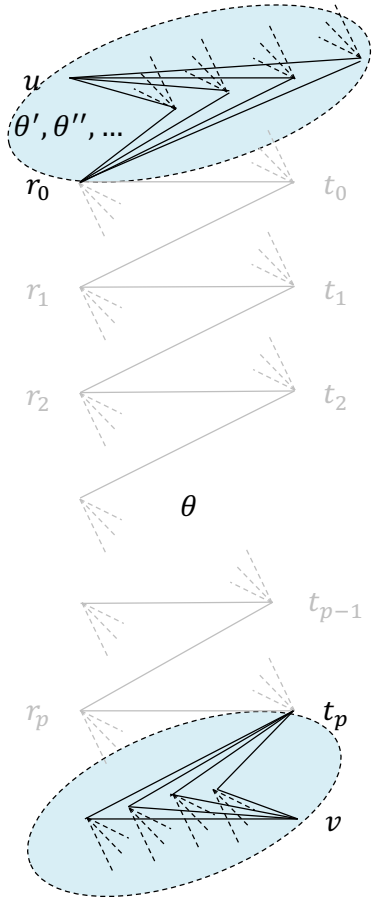

(b)

Parallel branches of $\theta^{\prime}$ such that: For all $\left(\alpha_{1}, \beta_{1}\right) \neq\left(\alpha_{2}, \beta_{2}\right)$ : $a_{\alpha_{1} \beta_{1}} b_{\alpha_{2} \beta_{2}} \neq a_{\alpha_{2} \beta_{2}} b_{\alpha_{1} \beta_{1}}$

Figure 3: Illustration of the Block $B^{(p)}(u, v)$ and the progression of assignments $\theta_{0}, \theta, \theta^{\prime}$ that, together, satisfy conditions (78), (79), and (80). Fig. (a) shows only the zig-zag part $B^{(p)}\left(r_{0}, t_{p}\right)$, where we choose $\theta$ to satisfy conditions (78) and (79). The assignment $\theta$ is independent of $\alpha, \beta$ and leaves unassigned some Boolean variables at the beginning and that the end ( $\left.u_{\alpha}, v_{\beta}\right)$. Fig. (b) extends the block $B^{(p)}\left(r_{0}, t_{p}\right)$ with a prefix and a suffix consisting of a single branch, thus $B^{(p)}(u, v)$ is isomorphic to $B^{(p+2)}\left(r_{0}, t_{p+2}\right)$. Here $\theta^{\prime}$ s extends the assignment $\theta$ to the remaining variables in the prefix and suffix in order to ensure one condition $a_{\alpha_{1} \beta_{1}} b_{\alpha_{2} \beta_{2}} \neq$ $a_{\alpha_{2} \beta_{2}} b_{\alpha_{1} \beta_{1}}$. Fig. (c) extends the prefix/suffix with parallel branches in order to satisfy all conditions $a_{\alpha_{1} \beta_{1}} b_{\alpha_{2} \beta_{2}} \neq a_{\alpha_{2} \beta_{2}} b_{\alpha_{1} \beta_{1}}$.

two sub-clauses $S_{J_{1}}, S_{J_{2}}$ in $C_{0}$ : since $Q$ is forbidden (see Def. C.10), each has a common symbol with $C_{1}$. We consider the grounding of $C_{0}$ that maps $S_{J_{1}}$ to the block $B\left(r_{i}, t_{i-1}\right)$, maps $S_{J_{2}}$ to the block $B\left(r_{i}, t_{i}\right)$, and maps each all other subclauses to distinct dead-end branches $B\left(r_{i}, e_{i}^{(j)}\right)$. This is possible because there are $m-2$ dead-end branches, and $m \geq \ell$, and it is also necessary, in order to ensure that the grounded clause is not redundant, see Example A.3 in Appendix A. Therefore, this grounding is a clause in $Y_{\alpha \beta}^{(p)}$ that is connected to $C_{1}\left(r_{i}, t_{i-1}\right)$ via $S_{J_{1}}\left(r_{i}, t_{i-1}\right)$ and is also connected to $C_{1}\left(r_{i}, t_{i}\right)$ via $S_{J_{2}}\left(r_{i}, t_{i}\right)$. By repeating this for all links of the zig-zag chain, we obtain a long sequence of connected clauses that start with $C_{1}\left(r_{0}, t_{0}\right)$, end with $C_{k-1}\left(r_{p}, t_{p}\right)$. With the same argument we can extend this connected component along all dead-end branches, i.e extend it with $C_{1}\left(r_{i}, e_{i}^{(j)}\right), \ldots, C_{k-1}\left(r_{i}, e_{i}^{(j)}\right)$ for all $i, j$, and similarly with $C_{k-1}\left(f_{i}^{(j)}, t_{i}\right), \ldots, C_{1}\left(f_{i}^{(j)}, t_{i}\right)$. Thus, this giant connected component has a zig-zag, with many spikes. It remains to prove that the clauses at the end of the zig-zag and at the end of the spikes are also connected. Now consider the beginning of the zig-zag, which is a grounding of $G_{\alpha}\left(r_{0}, t_{0}\right)$. When $\alpha \neq \hat{1}$, then, as we argued in the proof of Lemma C.15, no clause of $G_{\alpha}\left(r_{0}, t_{0}\right)$ can make $C_{1}\left(r_{0}, t_{0}\right)$ redundant, no middle clause can make any clause in $G_{\alpha}\left(r_{0}, t_{0}\right)$ redundant, and, while some clauses within $G_{\alpha}\left(r_{0}, t_{0}\right)$ might have homomorphisms to others, any remaining non-redundant clause of $G_{\alpha}\left(r_{0}, t_{0}\right)$ contains all ubiquitous symbols. The case when $\alpha=\hat{1}$ differs a little from Lemma C.15, and here we consider two cases. If some ubiquitous symbol of $Q$ occurs in a middle clause $C$, then all ubiquitous symbols of $Q$ occur in some middle clauses by Lemma C.19 (4). Since each such middle clauses is non-redundant in $C\left(r_{0}, t_{0}\right)$, and is connected to the path $C_{1}, C_{2}, \ldots$ (otherwise it consists only of ubiquitous symbols, hence there exists a homomorphism $C \rightarrow C_{0}$ ), the entire Boolean formula is connected and has all Boolean variables. If no ubiquitous symbol occurs in a middle clause, then every clause in $G_{\hat{1}}\left(r_{0}, t_{0}\right)$ is non-redundant, because if there were a homomorphism from some middle clause $C\left(r_{0}, t_{0}\right)$, then $C$ must contain only non-ubiquitous symbols, hence all are in $C_{1}$, by the Definition C.10 of forbidden queries, which implies a homomorphism $C \rightarrow C_{1}$, contradiction. 
Recall from Section B that the distance of two Boolean variables in a monotone formula is the smallest number of clauses that connects them. Fix a left-right path $C_{0}, C_{1}, \ldots, C_{k}$ in $Q$, of minimal length. Let $U$ be any left-ubiquitous symbol in $C_{0}$; recall that, by Lemma C.19, $U$ does not occur in $C_{1}$. Similarly, let $V$ be a right-ubiquitous symbol in $C_{k}$ and note that that it does not occur in $C_{k-1}$. We think of the Boolean variables (tuples) in the zig-zag block $B^{(p)}\left(r_{0}, t_{p}\right)$ as being partially ordered: $U\left(r_{0}, t_{0}\right)$ is the very "first" tuple, and $X$ comes before $Y$ if $d\left(U\left(r_{0}, t_{)}, X\right)<d\left(U\left(r_{0}, t_{0}\right), Y\right)\right.$ in the Boolean formula $Y_{\alpha \beta}^{(p)}$. This is only a pre-order, because we may have equal distances, it helps understanding the structure of the block $B^{(p)}\left(r_{0}, t_{p}\right)$. The next lemma shows that this pre-order is independent of the choice of $\alpha, \beta$.

Lemma C.25. Let $X$ denote the Boolean variable associated to any tuple of the zig-zag block $B\left(r_{0}, t_{p}\right)$. Then the distance between $U\left(r_{0}, t_{0}\right)$ and $X$ is the same in all formulas $Y_{\alpha \beta}^{(p)}$, i.e. it does not depend on $\alpha, \beta$. Similarly, the distance from $X$ to $V\left(r_{p}, t_{p}\right)$ is the same.

Proof. Every clause in $Y_{\alpha \beta}^{(p)}$ that contains $U\left(r_{0}, t_{0}\right)$ is connected to the clause $C_{1}\left(r_{0}, t_{0}\right)$ (and is not connected to $C_{2}\left(r_{0}, t_{0}\right)$ ). From there, the distance to any variable $X$ is the same, regardless of the choice of $\alpha, \beta$. The same argument applies to $Y_{\alpha \hat{1}}^{(p)}$ and $Y_{\hat{1} \beta}^{(p)}$.

Lemмa C.26. Let $X$ be the Boolean variable associated to a tuple on the main branch of the zig-zag block $B\left(r_{0}, t_{p}\right)$; that is, $X$ has the form $X=S\left(r_{i}, t_{i}\right)$ or $X=S\left(r_{i}, t_{i-1}\right)$, but not on a dead-end branch like $S\left(r_{i}, e_{i}^{(j)}\right)$. Assume that $d\left(U\left(r_{0}, t_{0}\right), X\right) \geq 2$ and $d\left(X, V\left(r_{p}, t_{p}\right)\right) \geq 2$. Then $X$ disconnects $U, V$ in $Y_{\alpha \beta}^{(p)}$, for all $\alpha, \beta$ (see Definition B.6).

Proof. Let $S$ be the relational symbol of the tuple $X$, and assume that $X=S\left(r_{i}, t_{i}\right)$; the case $X=S\left(r_{i}, t_{i-1}\right)$ is similar. Since $Q$ is final, both $Q[S:=0]$ and $Q[S:=1]$ are safe, and this can happen in one of two ways. The first is that the left clause $C_{0}$ and right clause $C_{k}$ remain left/right clauses in $Q[S:=0]$ (or $Q[S:=1]$ ), but the path $C_{1}, \ldots, C_{k-1}$ is disconnected, i.e. one of the clauses becomes true or becomes redundant. In that case setting $X:=0$ or $X:=1$ also disconnects the Boolean variables $U\left(r_{i}, t_{i}\right)$ from $V\left(r_{i}, t_{i}\right)$. The second case is when $Q[S:=0]$ or $Q[S:=1]$ has no left clause, or no right clause: this happens either because they become true, or redundant, or they degenerate to middle clauses (e.g. setting $S_{1}:=0$ in $\left.\forall y\left(U(x, y) \vee S_{1}(x, y)\right) \vee \forall y\left(U(x, y) \vee S_{2}(x, y)\right)\right)$. In that case the connection between $C_{1}\left(r_{i}, t_{i-1}\right)$ and $C_{1}\left(r_{i}, t_{i}\right)$ is broken, again disconnecting $U\left(r_{0}, t_{0}\right)$ from $V\left(r_{p}, t_{p}\right)$.

\section{C.7 Consistent Assignments}

We want to assign the same probabilities to similar tuples in similar blocks, e.g. the grounding of $S_{3}$ should have the same probability in all blocks $B\left(r_{0}, t_{0}\right), B\left(r_{1}, t_{1}\right), B\left(r_{2}, t_{2}\right), \ldots$ We make this precise below. We denote with lower case $s, s^{\prime}, \ldots$ the real variables representing the probabilities of the ground tuples, e.g. $S\left(r_{i}, t_{i}\right), S^{\prime}\left(r_{i}, t_{i}\right), \ldots$ We group the Boolean variables and their associated real variables into equivalence classes defined follows:

- For each symbol $S \in \mathcal{R}$ the odd equivalence class is the set of Boolean variables corresponding to its grounding in the odd-numbered zig-zag blocks: $S\left(r_{0}, t_{0}\right), S\left(r_{1}, t_{1}\right), S\left(r_{2}, t_{2}\right), \ldots, S\left(r_{p}, t_{p}\right)$.

- For each symbol $S \in \mathcal{R}$ the even equivalence class is the set of real variables corresponding to its grounding in the even-numbered zig-zag blocks: $S\left(r_{1}, t_{0}\right), S\left(r_{2}, t_{1}\right), S\left(r_{3}, t_{2}\right), \ldots, S\left(r_{p}, t_{p-1}\right)$.

- For each symbol $S \in \mathcal{R}$ the $j$ 'th left dead-end equivalence class is the set of variables corresponding to the $S\left(r_{1}, e_{1}^{(j)}\right), \ldots, S\left(r_{p}, e_{p}^{(j)}\right)$; similarly for the $j$ 'th right dead-end equivalence class, $S\left(f_{0}^{(j)}, t_{0}\right), S\left(f_{1}^{(j)}, t_{1}\right), \ldots, S\left(f_{p-1}^{(j)}, t_{p}\right)$.

- We will write $\ldots, S_{i-1}, S_{i}, S_{i+1}, \ldots$ for variables in the same equivalence class derived from some symbol $S$. We denote similarly the real variables, $\ldots, s_{i-1}, s_{i}, s_{i+1}, \ldots$ This notation is with some abuse, because does not specify whether we mean the odd equivalence class, or the even class, or one of the dead end classes. Depending on the type of class, it may contain either $p$ or $p+1$ variables.

- Finally, we notice that the distance between two consecutive variables in the same odd equivalence class or the same even equivalence class is exactly $2 k$, i.e. $d\left(S_{i-1}, S_{i}\right)=2 k, \forall i$. The distance between two consecutive variables in a dead-end equivalence class is $\geq 2 k$, but in general depends on the symbol $S$.

Definition C.27. Let $\theta$ be any partial assignment from the Boolean variables in the zig-zag block $B\left(r_{0}, t_{p}\right)$ to $\{0,1 / 2,1\}$. We say that $\theta$ is consistent if, for every equivalence class $E$ the following holds. If $X, Y$ are two variables in that equivalence class and $\theta(X)$ is defined, then either $\theta(Y)$ is also defined as $\theta(X)=\theta(Y)$, or $\theta(Y)$ is undefined and $Y=S\left(r_{0}, t_{0}\right)$ or $Y=S\left(r_{p}, t_{p}\right)$ for some symbol $S$.

In other words, $\theta$ must act in the same way on the entire equivalence class, with only exception that it may leave some variables in the first block $B\left(r_{0}, t_{0}\right)$ and the last block $B\left(r_{p}, t_{p}\right)$ undefined.

We define now a partial, consistent assignment $\theta_{0}$ as follows, see also Fig. 3 (a). Let $X$ be a tuple on a dead-end branch, e.g. $X=S\left(r_{i}, e_{i}^{(j)}\right)$. Setting $X:=0$ or $X:=1$ may disconnect $U\left(r_{0}, t_{0}\right)$ from $V\left(r_{p}, t_{p}\right)$, for example, if $X$ occurs in one grounding of $C_{0}$ that connects $C_{1}\left(r_{i}, t_{i-1}\right)$ with $C_{1}\left(r_{i}, t_{i}\right)$, then setting $X:=0$ or $X:=1$ may disconnect the main chain from $U\left(r_{0}, t_{0}\right)$ to $V\left(r_{p}, t_{p}\right)$. If $\operatorname{setting} X:=0$ or $X:=1$ does not disconnect $U\left(r_{0}, t_{0}\right)$ from $V\left(r_{p}, t_{p}\right)$, then we define $\theta_{0}(X):=0$, or 1 respectively, and do this for all tuples the equivalence class of $X$; it holds that the tuples $U\left(r_{0}, t_{0}\right)$ and $V\left(r_{p}, t_{p}\right)$ remain connected in all Boolean formulas $Y_{\alpha \beta}^{(p)}\left[\theta_{0}\right]$, for all $\alpha$, $\beta$. We proceed similarly with the 
dead-end tuples on the right, i.e. we set an entire equivalence class to 0 or to 1 if $U\left(r_{0}, t_{0}\right)$ and $V\left(r_{p}, t_{p}\right)$ remain connected. After this process, $\theta_{0}$ has the following property:

Definition C.28. Let $U, V$ be the left- and right-ubiquitous symbols introduced above. We say that the consistent partial assigning $\theta_{0}$ is final if, forall $\alpha, \beta$, the Boolean function $Y_{\alpha \beta}^{(p)}\left[\theta_{0}\right]$ is connected, and, for any tuple $X$ in $B\left(r_{0}, t_{p}\right)$, setting $X:=0$ or $X:=1$ disconnects $U\left(r_{0}, t_{0}\right)$ from $V\left(r_{p}, t_{p}\right)$.

We will fix $\theta_{0}$ from now on.

\section{C.8 The Eigenvalues of the Zig-zag Block}

We will now give a closed form formula for the probability of $Y_{\alpha \beta}^{(p)}$, which, recall, we are using temporarily to denote the lineage on the zig-zag block $B\left(r_{0}, t_{p}\right)$, see Eq. (83), i.e. without the prefix/suffix blocks. Since $\alpha \in L(\mathbf{G})$ and $\beta \in L(\mathbf{H})$, the probabilities $y_{\alpha \beta}^{(p)}$ form a $(\bar{m}+1) \times(\bar{n}+1)$ matrix. However, we show that this matrix has rank 2 , hence it can be described by a $2 \times 2$ matrix, and, as a consequence, $y_{\alpha \beta}^{(p)}$ is given in terms of two eigenvalues: $y_{\alpha \beta}^{(p)}=a_{\alpha \beta} \lambda_{1}^{p}+b_{\alpha \beta} \lambda_{2}^{p}$ for $\lambda_{1}, \lambda_{2} \neq 0$ and $\lambda_{1} \neq \pm \lambda_{2}$.

Let $C_{0}, C_{1}, \ldots, C_{k}$ be a left-right path in $Q$, of minimal length, and recall that $U, V$ are two ubiquitous symbols that do not occur in $C_{1}, C_{k-1}$. Fix an index $j$ such that $3 \leq j \leq k-5$, and fix a symbol $S \in \operatorname{Symb}\left(C_{j}\right) \cap \operatorname{Symb}\left(C_{j+1}\right)$. Since $Q$ is final, both $Q[S:=0]$ and $Q[S:=1]$ are safe queries, which implies that $S$ disconnects $Q_{\text {left }}$ from $Q_{\text {right }}$ in both $Q[S:=0]$ and $Q[S:=1]$. We will call $S$ an articulation symbol. We denote by $S_{0}, S_{1}, \ldots, S_{p}$ the Boolean variables forming the odd equivalence class $S\left(r_{0}, t_{0}\right), \ldots, S\left(r_{p}, t_{p}\right)$, and denote by $s_{0}, s_{1}, \ldots, s_{p}$ their associated real variables. In Fig. 3 (a) the articulation variables are shown with an $x$.

Denote by $B\left(r_{0}, r_{i}\right)$ and $B\left(t_{i}, t_{p}\right)$ the following subsets of the zig-zag block $B\left(r_{0}, t_{p}\right)$ :

$$
\begin{aligned}
& B\left(r_{0}, r_{i}\right) \stackrel{\text { def }}{=} \bigcup_{\ell=1, i}\left(B\left(r_{\ell-1}, t_{\ell-1}\right) \cup B\left(r_{\ell}, t_{\ell-1}\right) \cup \bigcup_{j}\left(B\left(r_{\ell}, e_{\ell}^{(j)}\right) \cup B\left(f_{\ell-1}^{(j)}, t_{\ell-1}\right)\right)\right) \\
& B\left(t_{i}, t_{p}\right) \stackrel{\text { def }}{=} \bigcup_{\ell=i+1, p}\left(B\left(r_{\ell}, t_{\ell-1}\right) \cup B\left(r_{\ell}, t_{\ell}\right) \cup \bigcup_{j}\left(B\left(f_{\ell-1}^{(j)}, t_{\ell-1}\right) \cup B\left(r_{\ell}, e_{\ell}^{(j)}\right)\right)\right)
\end{aligned}
$$

These are precisely the two connected components of $B\left(r_{0}, t_{p}\right)$ after we remove the single block $B\left(r_{i}, t_{i}\right)$.

Lemma C.29. The Boolean variable $S_{i}$ disconnects $B\left(r_{0}, r_{i}\right)$ and $B\left(t_{i}, t_{p}\right)$ (see Def. B.6) in the Boolean function $Y_{\alpha \beta}^{(p)}$, for any $\alpha, \beta$.

In other words, we have:

$$
Y_{\alpha \beta}^{(p)}\left[S_{i}:=0\right]=A_{0} \wedge B_{0} \quad Y_{\alpha \beta}^{(p)}\left[S_{i}:=1\right]=A_{1} \wedge B_{1}
$$

where all Boolean variables from $B\left(r_{0}, r_{i}\right)$ are in $A_{0}, A_{1}$, and all Boolean variables from $B\left(t_{i}, t_{p}\right)$ are in $B_{0}, B_{1}$. The proof follows immediately from the fact that $S$ disconnects $Q_{\text {left }}$ from $Q_{\text {right }}$ in both $Q[S:=0]$ and $S[S:=1]$.

Since $S_{\ell}$ is in $A_{0}, A_{1}$ for $\ell<i$, we can repeat this process and use $S_{\ell}$ to disconnect $A_{0}, A_{1}$, etc. More generally, consider any values $v_{0}, v_{1}, \ldots, v_{p} \in\{0,1\}$. Then, assigning these values to the variables of the articulation symbol splits $Y_{\alpha \beta}^{(p)}$ into $p+2$ formulas with no common Boolean variables.

$$
Y_{\alpha \beta}^{(p)}\left[S_{0}:=v_{0}, S_{1}:=v_{1}, \cdots, S_{p}:=v_{p}\right]=U_{\alpha}^{\left(v_{0}\right)} \wedge Z_{1}^{\left(v_{0} v_{1}\right)} \wedge \cdots \wedge Z_{p}^{\left(v_{p-1} v_{p}\right)} \wedge V_{\beta}^{\left(v_{p}\right)}
$$

The only expressions that depend on the choice of $\alpha, \beta$ are $U_{\alpha}^{\left(v_{0}\right)}$ and $V_{\beta}^{\left(v_{p}\right)}$. Since all Boolean expressions on the RHS in (85) have disjoint Boolean variables, their probabilities are independent, hence their arithmetization is a product of multilinear polynomials:

$$
y_{\alpha \beta}^{(p)}\left[s_{0}:=v_{0}, s_{1}:=v_{1}, \cdots, s_{p}:=v_{p}\right]=u_{\alpha}^{\left(v_{0}\right)} \cdot z_{1}^{\left(v_{0} v_{1}\right)} \cdots z_{p}^{\left(v_{p-1} v_{p}\right)} \cdot v_{\beta}^{\left(v_{p}\right)}
$$

We express the result in matrix form, where $\operatorname{diag}(a, b)$, denotes the diagonal matrix $\left[\begin{array}{cc}a & 0 \\ 0 & b\end{array}\right]$. This is an important expression which we define formally:

Definition C.30. The polynomial $y_{\alpha \beta}^{(p)}$ is defined as follows:

$$
y_{\alpha, \beta}^{(p)}=\left[\begin{array}{ll}
u_{\alpha}^{(0)} & u_{\alpha}^{(1)}
\end{array}\right] \cdot \operatorname{diag}\left(1-s_{0}, s_{0}\right) \cdot\left[\begin{array}{cc}
z_{1}^{(00)} & z_{1}^{(01)} \\
z_{1}^{(10)} & z_{1}^{(11)}
\end{array}\right] \cdot \operatorname{diag}\left(1-s_{1}, s_{1}\right) \cdot\left[\begin{array}{cc}
z_{2}^{(00)} & z_{2}^{(01)} \\
z_{2}^{(10)} & z_{2}^{(11)}
\end{array}\right] \ldots\left[\begin{array}{cc}
z_{p}^{(00)} & z_{p}^{(01)} \\
z_{p}^{(10)} & z_{p}^{(11)}
\end{array}\right] \cdot \operatorname{diag}\left(1-s_{p}, s_{p}\right)\left[\begin{array}{c}
v_{\beta}^{(0)} \\
v_{\beta}^{(1)}
\end{array}\right]
$$


Denote by $\mathrm{z}_{i}$ the $2 \times 2$ matrix:

$$
\mathbf{z}_{i} \stackrel{\text { def }}{=}\left[\begin{array}{ll}
z_{i}^{(00)} & z_{i}^{(01)} \\
z_{i}^{(10)} & z_{i}^{(11)}
\end{array}\right]
$$

We view it as matrix of multilinear polynomials, where the variables represent the (yet unchosen) probabilities of the tuples in all our blocks.

Our next task is to define a consistent assignment $\theta$ that extends $\theta_{0}$ from Sec. C.6, such that $\operatorname{det}\left(\mathrm{z}_{i}[\theta]\right) \neq 0$. For that we will use Lemma 1.2 in the introduction. However, we have a problem: the matrix $\mathbf{z}_{i}$ may contain two variables from the same equivalence class, and the assignment $\theta$ from Lemma 1.2 might assign them different values, making $\theta$ is inconsistent. This happens whenever the decomposition in Eq. (84) has migrating variables. Since we chose the articulation variable $S$ in $\operatorname{Symb}\left(C_{j}\right) \cap \operatorname{Symb}\left(C_{j+1}\right)$, the Boolean variable $S_{i}=S\left(r_{i}, t_{i}\right)$ disconnects $U_{i} \stackrel{\text { def }}{=} U\left(r_{i}, t_{i}\right), V_{i} \stackrel{\text { def }}{=} V\left(r_{i}, t_{i}\right)$, i.e. the left-ubiquitous symbol and the right-ubiquitous symbol in block $B\left(r_{i}, t_{i}\right)$. The distance from $U_{i}$ to $S_{i}$ is $j$, and the distance from $S_{i}$ to $V_{i}$ is $k-j-1$, hence, by Lemma B.10 item 2, $S_{i}$ also separates all the symbols in $C_{0}, \ldots, C_{j-2}$ from $C_{j+2}, \ldots, C_{k}$ (more precisely: their groundings in the block $B\left(r_{i}, t_{i}\right)$ ), hence the only possible migrating variables are of the form $S_{i}^{\prime} \stackrel{\text { def }}{=} S^{\prime}\left(r_{i}, t_{i}\right)$, with $S^{\prime} \in \operatorname{Symb}\left(C_{j-1}\right) \cup \cdots \cup \operatorname{Symb}\left(C_{j+1}\right)$. In other words, $d\left(S_{i}^{\prime}, S_{i}\right) \leq 1$. Suppose that $S_{i}^{\prime}$ migrates from left to right, i.e. it occurs in $A_{0}$ and in $B_{1}$ in Eq. (84). Then:

- $s_{i}^{\prime}$ occurs in the first column of $\mathbf{z}_{i}$, because this is part of $A_{0}$.

- $s_{i}^{\prime}$ occurs in the second row of $\mathbf{z}_{i+1}$, because this is part of $B_{0}$; then it's equivalent variable $s_{i-1}^{\prime}$ occurs in the second row of $\mathbf{z}_{i}$. If $S_{i}^{\prime}$ migrates from right to left, i.e. from $B_{1}$ to $A_{0}$, then:

- $s_{i}^{\prime}$ occurs in the first row of $\mathbf{z}_{i+1}$, because this is part of $B_{0}$; hence $s_{i-1}^{\prime}$ occurs in the first row of $\mathbf{z}_{i}$.

- $s_{i}^{\prime}$ occurs in the second column of $\mathbf{z}_{i}$, because this is part of $A_{1}$.

Pictorially, these two cases are illustrated as follows:

$$
\mathbf{z}_{i}=\left[\begin{array}{ll}
z_{i}^{(00)}\left(s_{i}^{\prime}\right) & z_{i}^{(01)} \\
z_{i}^{(10)}\left(s_{i-1}^{\prime}, s_{i}^{\prime}\right) & z_{i}^{(11)}\left(s_{i-1}^{\prime}\right)
\end{array}\right] \quad \mathbf{z}_{i}=\left[\begin{array}{ll}
z_{i}^{(00)}\left(s_{i-1}^{\prime}\right) & z_{i}^{(01)}\left(s_{i-1}^{\prime}, s_{i}^{\prime}\right) \\
z_{i}^{(10)} & z_{i}^{(11)}\left(s_{i}^{\prime}\right)
\end{array}\right]
$$

We seek a consistent assignment $\theta$, hence we need $\theta\left(s_{i-1}^{\prime}\right)=\theta\left(s_{i}^{\prime}\right)$, and for that we will simply substitute the real variable $s_{i-1}^{\prime}$ with the variable $s_{i}^{\prime}$. To do that, we need the following technical lemma.

Lemma C.31. Let $F$ be a connected Boolean function, and $\mathbf{A}=\left(A_{1}, \ldots, A_{k}\right), \mathbf{B}=\left(B_{1}, \ldots, B_{k}\right)$ be two tuples ofk Boolean variables each. Let $X$ be a variable that disconnects $\mathbf{A}, \mathbf{B}$, such that $d(\mathrm{~A}, X) \geq 3$ and $d(\mathbf{B}, X) \geq 3$. Let $f$ be the arithmetization of $F$. Then the polynomial $f[\mathbf{b}:=\mathbf{a}]$, where each variable $b_{i}$ is substituted by the variable $a_{i}$, is irreducible.

Notice that, while $f$ is a multilinear polynomial, each variable $a_{i}$ has degree 2 in the polynomial $f[\mathbf{b}:=\mathbf{a}]$. In general, if $f$ is irreducible, then equating variables does not keep it irreducible. For example, if $F=A \vee B$ then $f=a+b-a b$ and $f[b:=a]=2 a-a^{2}$ factorizes as $a(2-a)$. The lemma gives sufficient conditions for which $f[\mathbf{B}:=\mathbf{A}]$ is irreducible.

Proof. By Lemma B.10 (2) $X$ disconnects a ball around of A from a ball around B. Let $C, D$ be two variables s.t. $d(\mathbf{A}, C)=d(\mathbf{B}, D)=1$, thus, $X$ disconnects $\mathrm{A} C, \mathrm{~B} D$. Decompose the multilinear polynomial according to $X$ :

$$
\begin{aligned}
f[x:=0] & =g_{0}(\mathbf{a}, c) \cdot h_{0}(\mathbf{b}, d) & f[x:=1] & =g_{1}(\mathbf{a}, c) \cdot h_{1}(\mathbf{b}, d) \\
f[\mathbf{b}:=\mathbf{a}, x:=0] & =g_{0}(\mathbf{a}, c) \cdot h_{0}(\mathbf{a}, d) & f[\mathbf{b}:=\mathbf{a}, x:=1] & =g_{1}(\mathbf{a}, c) \cdot h_{1}(\mathbf{a}, d)
\end{aligned}
$$

where we indicated that a, $c$ occurs only in $g_{0}, g_{1}$, while $\mathbf{b}, d$ occurs only in $h_{0}, h_{1}$. While $g_{0}(\mathbf{a}, c)$ may further factorize, all variables a, $c$ are in the same irreducible factor, because their clauses are connected in $F[X:=0]$; similarly for the other three expressions. Assume now that $f[\mathbf{b}:=\mathbf{a}]$ factorizes; since the degree of $X$ in $f[\mathbf{b}:=\mathbf{a}]$ is $\leq 1$, there exists an irreducible factor $k$ of $f[\mathbf{b}:=\mathbf{a}]$ that does not contain $X$. The $k$ divides both expressions in the second line above. We consider three cases.

Case 1: If $k$ contains the variable $c$, then it must divide both $g_{0}(\mathbf{a}, c)$ and $g_{1}(\mathbf{a}, c)$. It follows that $k$ divides $f=g_{0} \cdot h_{0} \cdot(1-x)+g_{1} \cdot h_{1} \cdot x$. This is a contradiction because $f$ is irreducible.

Case 2: If $k$ contains the variable $d$, the it must divide $h_{0}(\mathbf{a}, d)$ and $h_{1}(\mathbf{a}, d)$. In particular $k$ contains the variables a, and the degrees of all a-variables is 1 , because their degree in $h_{0}(\mathbf{a}, d), h_{1}(\mathbf{a}, d)$ is 1 . Substitute $\mathbf{b}$ for $\mathbf{a}$ in $k$, we a conclude that $k$ [a $\left.:=\mathbf{a}\right]$ divides both $h_{0}(\mathbf{b}, d)$ and $h_{1}(\mathbf{b}, d)$. Hence it divides $f$ by the argument in Case 1 , and we reached a contradiction.

Case 3 If $k$ contains neither $c$ nor $d$, then by the argument above it cannot contain any of a. Since $k$ divides $g_{0}(\mathbf{a}, c) \cdot h_{0}(\mathbf{a}, d)$, it also divides $g_{0}(\mathbf{a}, c) \cdot h_{0}(\mathbf{b}, d)$, and it similarly divides $g_{1}(\mathbf{a}, c) \cdot h_{1}(\mathbf{b}, d)$. This, again, implies that $k$ divides $f$, which is a contradiction.

We will now prove the existence of a consistent assignment $\theta$ that extends $\theta_{0}$ such that $\operatorname{det}\left(\mathbf{z}_{i}[\theta]\right) \neq 0$. Recall that $\theta_{0}$, introduced in the previous section, has the property that all Boolean functions $Y_{\alpha \beta}^{(p)}\left[\theta_{0}\right]$ are connected, and every variable $X$ disconnects $U\left(r_{0}, t_{0}\right)$ from $V\left(r_{p}, t_{p}\right)$. 
Theorem C.32. Let $\mathbf{V} \stackrel{\text { def }}{=} \bigcup_{i} \operatorname{Vars}\left(\mathbf{z}_{i}\right) \cup\left\{s_{0}, s_{1}, \ldots, s_{p}\right\}$, and define $\theta$ the following assignment: if $\theta_{0}(x)$ is defined then $\theta(x) \stackrel{\text { def }}{=} \theta_{0}(x)$, otherwise $\theta(x) \stackrel{\text { def }}{=} 1 / 2$. Then, for every $i, \operatorname{det}\left(\mathbf{z}_{i}[\theta]\right) \neq 0$.

Proof. Choose arbitrary $\alpha, \beta$, and recall that the polynomial $y_{\alpha \beta}^{(p)}\left[\theta_{0}\right]$ is irreducible (Lemma C.24). Fix $i=1, \ldots, p$ : we will first define some consistent $\theta$ that extends $\theta_{0}$ such that $\operatorname{det}\left(\mathbf{z}_{i}[\theta]\right) \neq 0$. Denote by a the set of migrating variables $s_{i}^{\prime}$, and denote by $\mathbf{b}$ the set of the predecessor equivalent variables $s_{i-1}^{\prime}$; as we have seen, both $s_{i}^{\prime}$ and $s_{i-1}^{\prime}$ belong to $\mathbf{z}_{i}$. Let $\mathrm{A}, \mathrm{B}$ their corresponding Boolean variables. To apply Lemma C.31, we need to find some variable $X$ that disconnects A, B and that is far from both. Using the left-right path $C_{0}, C_{1}, \ldots, C_{k}$, choose any symbol $S^{\prime \prime} \in C_{j}$ for any $1<j<k-1$, and define $X \stackrel{\text { def }}{=} S^{\prime \prime}\left(r_{i}, t_{i-1}\right)$. Since all B-variables are in the block $B\left(r_{i-1}, t_{i-1}\right)$ and all A-variables are in $B\left(r_{i}, t_{i}\right)$, we have $d(\mathbf{B}, X) \geq 2$ and $d(\mathbf{A}, X) \geq 2$. Therefore, by the lemma, the polynomial $y_{\alpha \beta}^{(p)}\left[\mathbf{b}:=\mathbf{a}, \theta_{0}\right]$ is irreducible.

Claim 6. $\operatorname{det}\left(\mathbf{z}_{i}\left[\mathbf{b}:=\mathbf{a}, \theta_{0}\right]\right) \not \equiv 0$.

Proof. Assume the contrary, that $\operatorname{det}\left(\mathbf{z}_{i}\left[\mathbf{b}:=\mathbf{a}, \theta_{0}\right]\right) \equiv 0$. Then, by Theorem B.1, the matrix $\mathbf{y}_{i}\left[\mathbf{b}:=\mathbf{a}, \theta_{0}\right]$ has rank 1 , more precisely there exists polynomials $g_{0}, g_{1}, h_{0}, h_{1}$, such that:

$$
\left[\begin{array}{l}
g_{0} \\
g_{1}
\end{array}\right] \cdot\left[\begin{array}{ll}
h_{0} & h_{1}
\end{array}\right] \equiv \mathbf{z}_{i}\left[\mathbf{b}:=\mathbf{a}, \theta_{0}\right]
$$

Substituting this expression in $y_{\alpha \beta}^{(p)}\left[\mathbf{b}:=\mathbf{a}, \theta_{0}\right]$ we obtain a factorization:

$$
y_{\alpha_{0} \beta_{0}}^{(p)}\left[\mathbf{b}:=\mathbf{a}, \theta_{0}\right]=\underbrace{\left[\begin{array}{cc}
u_{\alpha_{0}}^{(0)} & u_{\alpha_{0}}^{(1)}
\end{array}\right] \ldots\left[\begin{array}{l}
g_{0} \\
g_{1}
\end{array}\right]}_{\text {factor } 1} \cdot \underbrace{\left[\begin{array}{ll}
h_{0} & h_{1}
\end{array}\right] \cdots\left[\begin{array}{c}
v_{\beta}^{(0)} \\
v_{\beta}^{(1)}
\end{array}\right]}_{\text {factor } 2}
$$

This contradicts the fact that $y_{\alpha \beta}^{(p)}\left[\mathbf{b}:=\mathbf{a}, \theta_{0}\right]$ is irreducible. This completes the proof of the claim.

CLAIM 7. There exists an assignment $\theta_{i}$ of the variables of $\mathbf{z}_{i}[\mathbf{b}:=\mathbf{a}]$ with values in $\{0,1 / 2,1\}$ s.t. $\theta_{i}$ extends $\theta_{0}$ and $\operatorname{det}\left(\mathbf{z}_{i}\left[\mathbf{b}::=\mathbf{a}, \theta_{i}\right]\right) \neq 0$.

Proof. We use Lemma 1.2 in the introduction. To apply it we must verify that $\operatorname{det}\left(\mathbf{z}_{i}\left[\mathbf{b}:=\mathbf{a}, \theta_{0}\right]\right)$ is a polynomial of $\operatorname{degree} \leq 2$ in each variable. This follows immediately by inspecting Eq. (86). When $s_{i}^{\prime}$ migrates from left to right then the variable $s_{i}^{\prime}=s_{i-1}^{\prime}$ has degree 2 in $y_{i}^{(10)}$ and degree 0 in $y_{i}^{(01)}$, hence it has degree $2 \operatorname{in} \operatorname{det}\left(\mathbf{z}_{i}\right)$, and similarly for variables that migrate from right to left. Therefore, by Lemma 1.2 , there exists an assignment $\theta_{i}$ of the variables in $\mathbf{z}_{i}\left[\mathbf{b}:=\mathbf{a}, \theta_{0}\right]$ (hence: an extension of $\left.\theta_{0}\right)$ such that $\operatorname{det}\left(\mathbf{z}_{i}\left[\mathbf{b}:=\mathbf{a}, \theta_{i}\right]\right) \neq 0$.

From here we derive immediately:

CLAIM 8. There exists a consistent assignment $\theta$ of the variables $\mathbf{V}$ that extends $\theta_{0}$ and $\operatorname{det}\left(\mathbf{z}_{i}[\theta]\right) \neq 0$ for all $i=1, p$.

Proof. Take $\theta=\theta_{1} \cup \theta_{2} \cup \cdots \cup \theta_{p}$. While $\theta_{i-1}, \theta_{i}$ are defined on some common variables (the migrating variables) they have the same values. Finally, assign $\theta\left(s_{0}\right)=\theta\left(s_{1}\right)=\cdots=\theta\left(s_{p}\right)=1 / 2$ (since the separator variables are not part of any matrix $\mathbf{z}_{i}$ ).

Finally, we prove that $\theta$ assigns the value $1 / 2$ to every variable not in the domain of $\theta_{0}$.

Claim 9. Assume $p \geq 3$, and let $\theta$ be any consistent assignment of the variables $\mathrm{V}$ that extends $\theta_{0}$. Suppose that there exists some variable $X \notin \operatorname{Dom}\left(\theta_{0}\right)$, such $\theta(X)=0$ or $\theta(X)=1$. Then there exists $i$ such that $\operatorname{det}\left(\mathrm{z}_{i}[\theta]\right)=0$.

Proof. Let $X$ be any variable $\notin \operatorname{Dom}\left(\theta_{0}\right)$. Consider the product of matrices in Def. C.30 that defines the polynomial $y_{\alpha, \beta}^{(p)}$; then $y_{\alpha, \beta}^{(p)}\left[\theta_{0}\right]$ is the same product, where $\theta_{0}$ is applied to each matrix, i.e. $z_{i}\left[\theta_{0}\right] . X$ is a variable that occurs in either one, or at most two consecutive matrices (when it migrates). Assuming it occurs in $\mathbf{z}_{i}, \mathbf{z}_{i+1}$, and we split the expression in Def. C.30 into three parts

Where:

$$
y_{\alpha, \beta}^{(p)}\left[X:=0, \theta_{0}\right]=\mathbf{a} \cdot \operatorname{diag}\left(1-s_{i-1}, s_{i-1}\right) \cdot \mathbf{w} \cdot \operatorname{diag}\left(1-s_{i+1}, s_{i+1}\right) \cdot \mathbf{b}
$$

$$
\mathbf{a} \stackrel{\text { def }}{=} \mathbf{u} \cdot \prod_{\ell=1, i-1} \operatorname{diag}\left(1-s_{\ell-1}, s_{\ell-1}\right) \cdot \mathbf{z}_{\ell}\left[\theta_{0}\right] \quad \mathbf{w} \stackrel{\text { def }}{=} \mathbf{z}_{i}\left[X:=0, \theta_{0}\right] \cdot \operatorname{diag}\left(1-s_{i}\right) \cdot \mathbf{z}_{i+1}\left[X:=0, \theta_{0}\right] \quad \mathbf{b} \stackrel{\operatorname{def}}{=} \prod_{\ell=i+2, p} \mathbf{z}_{\ell}\left[\theta_{0}\right] \cdot \operatorname{diag}\left(1-s_{\ell}, s_{\ell}\right) \cdot \mathbf{v}
$$

We prove that, if $y_{\alpha, \beta}^{(p)}\left[X:=0, \theta_{0}\right]$ factorizes, then $\operatorname{det}(\mathbf{w}) \equiv 0$. This $\operatorname{implies}$ that $\operatorname{either} \operatorname{det}\left(\mathbf{z}_{i}\left[X:=0, \theta_{0}\right]\right) \equiv 0$ or $\operatorname{det}\left(\mathbf{z}_{i+1}\left[X:=0, \theta_{0}\right]\right) \equiv 0$. We will denote $s \stackrel{\text { def }}{=} s_{i-1}$ and $s^{\prime} \stackrel{\text { def }}{=} s_{i+2}$ to reduce clutter, thus:

$$
y_{\alpha, \beta}^{(p)}\left[X:=0, \theta_{0}\right]=a_{0} b_{0} w_{00}(1-s)\left(1-s^{\prime}\right)+a_{0} b_{1} w_{01}(1-s) s^{\prime}+a_{1} b_{0} w_{10} s\left(1-s^{\prime}\right)+a_{1} b_{1} w_{01} s s^{\prime}
$$


Since $y_{\alpha, \beta}^{(p)}\left[X:=0, \theta_{0}\right]$ is reducible, let $f$ be an irreducible factor $f$ that contains the variable $U\left(r_{0}, t_{0}\right)$, where $U$ is a left ubiquitous symbol. That is, $f$ contains a "far left" variable, which only occurs in $a_{0}$ and $a_{1}$. In particular, it does not share any variables with $b_{0}, b_{1}$, because $X$ separates them in the Boolean formula $Y_{\alpha, \beta}^{(p)}\left[\theta_{0}\right]$. We have:

$$
f \mid a_{0} b_{0} w_{00}(1-s)\left(1-s^{\prime}\right)+a_{0} b_{1} w_{01}(1-s) s^{\prime}+a_{1} b_{0} w_{10} s\left(1-s^{\prime}\right)+a_{1} b_{1} w_{01} s^{\prime}
$$

Consider now the variables $s, s^{\prime}: f$ may contain neither, or just $s$, or both $s, s^{\prime}$ (since $U\left(r_{0}, t_{0}\right)$ is closer to $S_{i-1}$ than to $\left.S_{i+2}\right)$ in the latter case we switch the roles of $\mathbf{a}, \mathbf{b}$, i.e. start with some irreducible factor that contains some "far right" variable $V\left(r_{p}, t_{p}\right)$. Hence we will assume w.l.o.g. that $f$ does not contain $s^{\prime}$. Then, can set separately $s^{\prime}=0$ and $s^{\prime}=1$ and obtain:

$$
\begin{aligned}
& f \mid\left(a_{0} w_{00}(1-s)+a_{1} w_{10} s\right) b_{0} \\
& f \mid\left(a_{0} w_{01}(1-s)+a_{1} w_{11} s\right) b_{1}
\end{aligned}
$$

Consider first the case when $f$ does not contain $s$ either. In that case we repeat the argument, and obtain:

$$
f\left|a_{0} b_{0} w_{00} \quad f\right| a_{0} b_{1} w_{01} \quad f\left|a_{1} b_{0} w_{10} \quad f\right| a_{1} b_{1} w_{00}
$$

It follows that $f \mid a_{0}$ and $f \mid a_{1}$, which implies $f \mid y_{\alpha, \beta}^{(p)}\left[\theta_{0}\right]$ (without setting $X:=0$ ), which contradicts our assumption. Consider now the case when $f$ contains $s$. Notice that we can remove $b_{0}, b_{1}$ from (87), since $f$ shares no variables with them. We eliminate $a_{1}$ by multiply the first line by $w_{11}$ and the second by $-w_{10}$, then eliminate $a_{0}$ similarly, and obtain:

$$
\begin{aligned}
& f \mid a_{0}(1-s)\left(w_{00} w_{11}-w_{01} w_{10}\right) \\
& f \mid a_{1} s\left(w_{00} w_{11}-w_{01} w_{10}\right)
\end{aligned}
$$

If $\left(w_{00} w_{11}-w_{01} w_{10}\right) \neq 0$, then $f$ divides both $a_{0}(1-s)$ and $a_{1} s$, again implying that $f \mid y_{\alpha, \beta}^{(p)}\left[\theta_{0}\right]$, which is a contradiction.

In summary, we have

$$
\forall i: \quad \mathbf{z}_{i}[\theta] \stackrel{\text { def }}{=} \mathbf{z}=\left[\begin{array}{cc}
z_{00} & z_{01} \\
z_{10} & z_{11}
\end{array}\right]
$$

LEMMA C.33. $z_{00}, z_{01}, z_{10}, z_{11}>0$.

Proof. Consider $z_{00}$ : this is the probability of the Boolean expression $Z_{i}^{(00)}\left[\theta_{0}\right]$ in (85), for any choice of $i$. We claim that $Z_{i}^{(00)}\left[\theta_{0}\right] \not \equiv$ false. By definition, $Z_{i}^{(00)}\left[\theta_{0}\right]$ is the formula obtained by factorizing:

$$
Y_{\alpha \beta}^{(p)}\left[S_{i-1}:=0, S_{i}:=0, \theta_{0}\right]=A_{0} \wedge Z_{i}^{(00)}\left[\theta_{0}\right] \wedge B_{0}
$$

We claim that this expression is not $\equiv$ false. Indeed, by definition of $\theta_{0}, Y_{\alpha \beta}^{(p)}\left[\theta_{0}\right]$ is a connected monotone Boolean function, and by our choice of the variables $S_{i}$, their distance is $d\left(S_{i-1}, S_{i}\right)=2 k$, hence they are neither prime implicants, nor do they occur together in a clause. It follows that by setting both to 0 we not make $Y_{\alpha \beta}^{(p)}\left[S_{i-1}:=0, S_{i}:=0, \theta_{0}\right] \equiv$ false. Since all Boolean variables in $Z_{i}^{(00)}\left[\theta_{0}\right]$ have been assigned probability $1 / 2$, it follows that its probability is $>0$, proving $z_{00}>0$. The other cases are similar and omitted.

Denote by $\lambda_{1}, \lambda_{2}$ the eigenvalues of the matrix z. We also assign probabilities $1 / 2$ to all the articulation points: $s_{1}=s_{2}=\cdots=s_{p}=1 / 2$, and obtain:

$$
y_{\alpha \beta}^{(p)}[\theta]=\frac{1}{2^{p+1}}\left[\begin{array}{cc}
u_{\alpha}^{(0)} & u_{\alpha}^{(1)}
\end{array}\right] \cdot\left[\begin{array}{ll}
z_{00} & z_{01} \\
z_{10} & z_{11}
\end{array}\right]^{p} \cdot\left[\begin{array}{c}
v_{\beta}^{(0)} \\
v_{\beta}^{(1)}
\end{array}\right]=\frac{1}{2}\left(a_{\alpha \beta}\left(\lambda_{1} / 2\right)^{p}+b_{\alpha \beta}\left(\lambda_{2} / 2\right)^{p}\right)
$$

where $a_{\alpha \beta}, b_{\alpha \beta}$ are coefficients that are independent of $p$. We prove now Condition (78):

Theorem C.34. The following hold: $0<\left|\lambda_{1}\right|<\lambda_{2}$.

Proof. $\operatorname{det}(\mathbf{z}) \neq 0$ implies $\lambda_{1}, \lambda_{2} \neq 0 . \lambda_{1} \neq \lambda_{2}$, because both $z_{01}$ and $z_{10}$ are $\neq 0$, since they represent probabilities. $\lambda_{1}+\lambda_{2}>0$, because the trace of the matrix is $z_{00}+z_{11}>0$ as a sum of two probabilities.

Next, prove condition (79).

Lemma C.35. Assume $\lambda_{1}<\lambda_{2}$, then, for all $\alpha, \beta, b_{\alpha \beta}>0$. 
Proof. We first expand the power $p$ of the matrix $\mathbf{z}$ :

$$
\mathbf{z}^{p}=\left[\begin{array}{cc}
a_{1} \lambda_{1}^{p}+a_{2} \lambda_{2}^{p} & b_{1} \lambda_{1}^{p}+b_{2} \lambda_{2}^{p} \\
c_{1} \lambda_{1}^{p}+c_{2} \lambda_{2}^{p} & d_{1} \lambda_{1}^{p}+d_{2} \lambda_{2}^{p}
\end{array}\right]
$$

We prove that $a_{2}, b_{2}, c_{2}, d_{2}>0$. We start by observing that $\lambda_{1}, \lambda_{2}$ are the solutions to:

$$
\lambda^{2}-\left(z_{00}+z_{11}\right) \lambda+\left(z_{00} z_{11}-z_{01} z_{10}\right)=0
$$

We use the fact that the quantities $z_{00}, z_{01}, z_{10}, z_{11}$ represent proabilities, hence they are $>0$. It follows that $\lambda_{1}+\lambda_{2}=\operatorname{tr}(\mathbf{z})=z_{00}+z_{11}>0$. Since $\lambda_{1} \lambda_{2}=\operatorname{det}(\mathbf{z})=\left(z_{00} z_{11}-z_{01} z_{10}\right)$, we cannot have $\lambda_{1}=z_{00}$, because then $\lambda_{2}=\operatorname{tr}(\mathbf{z})-\lambda_{1}=z_{11}$ and $z_{00} z_{11}=\lambda_{1} \lambda_{2}=\operatorname{det}(\mathbf{z})=z_{00} z_{11}-z_{10} z_{01}$ implying $z_{01}=0$ or $z_{10}=0$, which is impossible by Lemma C.33. Thus, $\lambda_{1,2} \neq z_{00}, z_{11}$.

Next, since $\mathbf{z}^{0}$ is the identity matrix, we obtain:

$$
\begin{array}{ll}
a_{1}+a_{2}=1 & b_{1}+b_{2}=0 \\
c_{1}+c_{2}=0 & d_{1}+d_{2}=1
\end{array}
$$

It follows that $b_{1}=-b_{2}$ and $c_{1}=-c_{2}$. Similarly, we examine $z^{1}=z$, and deduce $a_{1} \lambda_{1}+a_{2} \lambda_{2}=z_{00}, d_{1} \lambda_{1}+d_{2} \lambda_{2}=z_{11}$. If $a_{1}=0$ then $a_{2}=1-a_{1}=1$ which implies $\lambda_{2}=z_{00}$, contradiction. Thus, $a_{1}, a_{2}, d_{1}, d_{2} \neq 0$. Finally, we consider the expression for $\mathbf{z}^{p}$ :

$$
\mathbf{z}^{p}=\left[\begin{array}{ll}
a_{1} \lambda_{1}^{p}+a_{2} \lambda_{2}^{p} & b_{2}\left(\lambda_{2}^{p}-\lambda_{1}^{p}\right) \\
c_{2}\left(\lambda_{2}^{p}-\lambda_{2}^{p}\right) & d_{1} \lambda_{1}^{p}+d_{2} \lambda_{2}^{p}
\end{array}\right]
$$

We notice that all entries in $\mathrm{z}^{p}$ are $>0$, because they are probabilities. When $p \rightarrow \infty$ then all terms above are dominated by $\lambda_{2}^{p}$, which implies $a_{2}, b_{2}, c_{2}, d_{2}>0$. Finally, the lemma follows from computing $y_{\alpha \beta}^{(p)}$ using (89), and obtain:

$$
y_{\alpha \beta}^{(p)}=\frac{1}{2}\left(\left(u_{\alpha}^{(0)} a_{1} v_{\beta}^{(0)}+u_{\alpha}^{(0)} b_{1} v_{\beta}^{(1)}+u_{\alpha}^{(1)} c_{1} v_{\beta}^{(0)}+u_{\alpha}^{(1)} d_{1} v_{\beta}^{(1)}\right) \lambda_{1}^{p}+\left(u_{\alpha}^{(0)} a_{2} v_{\beta}^{(0)}+u_{\alpha}^{(0)} b_{2} v_{\beta}^{(1)}+u_{\alpha}^{(1)} c_{2} v_{\beta}^{(0)}+u_{\alpha}^{(1)} d_{2} v_{\beta}^{(1)}\right) \lambda_{2}^{p}\right)
$$

and the lemma follows by observing that the factor of $\lambda_{2}^{p}$ is $>0$.

Finally, it remains to prove condition-(80): $a_{\alpha_{1} \beta_{1}} b_{\alpha_{2} \beta_{2}} \neq a_{\alpha_{2} \beta_{2}} b_{\alpha_{1} \beta_{1}}$. To enforce this condition we need to design carefully the prefix and suffix blocks. We do this in the next sections.

\section{C.9 One Condition $a_{\alpha_{1} \beta_{1}} b_{\alpha_{2} \beta_{2}} \neq a_{\alpha_{2} \beta_{2}} b_{\alpha_{1} \beta_{1}}$}

Our end goal is to define the block $B^{(p)}(u, v)$, and its probabilities, such as to satisfy all three conditions (78), (79), and (80). In the previous sections we have denoted by $Y_{\alpha \beta}^{(p)}$ and $y_{\alpha \beta}^{(p)}$ the lineage on the zig-zag block only (83), and satisfied the first two conditions; in this and the next section we extend it with the prefix/suffix, and satisfy the third condition, (80). Recall that the complete block $B^{(p)}(u, v)$ is:

$$
B^{(p)}(u, v)=B\left(u, r_{0}\right) \cup\left(\bigcup_{j} B\left(r_{0}, e_{0}^{(j)}\right)\right) \cup B\left(r_{0}, t_{p}\right) \cup\left(\bigcup_{j} B\left(f_{p}^{(j)}, t_{p}\right)\right) \cup B\left(t_{p}, v\right)
$$

We will denote by:

$$
\begin{gathered}
Y_{\alpha \beta}^{(p)}\left(r_{0}, t_{p}\right) \stackrel{\text { def }}{=} \Phi_{B\left(r_{0}, t_{p}\right)}\left(G_{\alpha}\left(r_{0}\right) \wedge Q \wedge H_{\beta}\left(t_{p}\right)\right) \\
Y_{\alpha \beta}^{(p)}(u, v) \stackrel{\text { def }}{=} \Phi_{B^{(p)}(u, v)}\left(G_{\alpha}(u) \wedge Q \wedge H_{\beta}(v)\right)
\end{gathered}
$$

and similarly for their probabilities, $y_{\alpha \beta}^{(p)}\left(r_{0}, t_{p}\right), y_{\alpha \beta}^{(p)}(u, v)$. We have already defined a partial assignment $\theta$ of Boolean variables in $B^{(p)}\left(r_{0}, t_{p}\right)$ to probabilities in $\{0,1 / 2,1\}$ to satisfy Condition (78) (Condition (79) came almost for free). Now we will extend $\theta$ to a total assignment, i.e. to all variables in the block $B^{(p)}(u, v)$, to also satisfy condition (80).

More precisely, let $\mathbf{V} \subset B\left(r_{0}, t_{p}\right)$ be the set of all Boolean variables that occur in the matrices $\mathbf{z}_{1}, \ldots, \mathbf{z}_{p}$. Add to it the articulation variables, $\mathbf{V}^{\prime} \stackrel{\text { def }}{=} \mathbf{V} \cup\left\{s_{0}, s_{1}, \ldots, s_{p}\right\}$. Then $\theta$ is the assignment of the variables $\mathbf{V}^{\prime}$ given by Theorem C.32. We have:

$$
\begin{aligned}
& y_{\alpha \beta}^{(p)}\left(r_{0}, t_{p}\right)[\theta]=\frac{1}{2^{p}}\left[\begin{array}{ll}
u_{\alpha}^{(0)}\left(r_{0}, t_{0}\right)[\theta] & u_{\alpha}^{(1)}\left(r_{0}, t_{0}\right)[\theta]
\end{array}\right] \cdot\left[\begin{array}{ll}
z_{00} & z_{01} \\
z_{10} & z_{11}
\end{array}\right]^{p} \cdot\left[\begin{array}{c}
v_{\beta}^{(0)}\left(r_{p}, t_{p}\right)[\theta] \\
v_{\beta}^{(1)}\left(r_{p}, t_{p}\right)[\theta]
\end{array}\right]=a_{\alpha \beta}\left(r_{0}, t_{p}\right)\left(\lambda_{1} / 2\right)^{p}+b_{\alpha \beta}\left(r_{0}, t_{p}\right)\left(\lambda_{2} / 2\right)^{p} \\
& y_{\alpha \beta}^{(p)}(u, v)[\theta]=\frac{1}{2^{p}}\left[\begin{array}{ll}
u_{\alpha}^{(0)}\left(u, t_{0}\right)[\theta] & u_{\alpha}^{(1)}\left(u, t_{0}\right)[\theta]
\end{array}\right] \cdot\left[\begin{array}{ll}
z_{00} & z_{01} \\
z_{10} & z_{11}
\end{array}\right]^{p} \cdot\left[\begin{array}{c}
v_{\beta}^{(0)}\left(r_{p}, v\right)[\theta] \\
v_{\beta}^{(1)}\left(r_{p}, v\right)[\theta]
\end{array}\right]=a_{\alpha \beta}\left(t_{p}, v\right)\left(\lambda_{1} / 2\right)^{p}+b_{\alpha \beta}\left(t_{p}, v\right)\left(\lambda_{2} / 2\right)^{p}(92)
\end{aligned}
$$


We are interested in the latter expression, where we haven't yet defined the structure and/or probabilities of the two polynomial matrices $\mathbf{u}_{\alpha}, \mathbf{v}_{\beta}$. Notice that, whatever our choice, conditions (78), (79) continue to apply, since we proved them for any polynomials $\mathbf{u}_{\alpha}, \mathbf{v}_{\beta}$. Now, we will design the prefix/suffix blocks and assign probabilities to the variables in $\mathbf{u}_{\alpha}, \mathbf{v}_{\beta}$ to also satisfy condition (80). We will do this in two steps. The first step, described in this section, will satisfy the condition for one pair $\left(\alpha_{1}, \beta_{1}\right) \neq\left(\alpha_{2}, \beta_{2}\right)$. For that we need a single branch in the prefix and a single branch in the suffix, thus $B^{(p)}(u, v)$ will be like $B^{(p+2)}\left(r_{0}, t_{p+2}\right)$, as illustrated in Fig. 3 (b). The second step, described in the next sections, uses multiple parallel branches to satisfy the condition for all pairs, illustrated in Fig. 3 (c).

We will start by satisfying a single condition: for a fixed pair $\left(\alpha_{1}, \beta_{1}\right) \neq\left(\alpha_{2}, \beta_{2}\right)$,our goal is to satisfy. $a_{\alpha_{1} \beta_{1}} b_{\alpha_{2} \beta_{2}} \neq a_{\alpha_{2} \beta_{2}} b_{\alpha_{1} \beta_{1}}$. We will use a single branch for the prefix/suffix blocks, hence $B^{(p)}(u, v)$ is isomorphic to $B^{(p+2)}\left(r_{0}, t_{p+2}\right)$, and, $y_{\alpha \beta}^{(p)}$ is given by (91). It suffices to prove how complete the assignment $\theta$ to all variables in $B^{(p)}\left(r_{0}, t_{p}\right)$ in order to satisfy $a_{\alpha_{1} \beta_{1}} b_{\alpha_{2} \beta_{2}} \neq a_{\alpha_{2} \beta_{2}} b_{\alpha_{1} \beta_{1}}$. Our construction is independent of $p$ because, on one hand, the condition that we need to satisfy, $a_{\alpha_{1} \beta_{1}} b_{\alpha_{2} \beta_{2}} \neq a_{\alpha_{2} \beta_{2}} b_{\alpha_{1} \beta_{1}}$, is independent of $p$, on the other hand we can assume w.l.o.g. that the polynomials $\mathbf{v}_{\beta}\left(r_{p}, t_{p}\right)[\theta]$ and $\mathbf{v}_{\beta}\left(r_{p+1}, t_{p+1}\right)[\theta]$ have the same variables, since the blocks $B\left(r_{p}, t_{p}\right)$ and $B\left(r_{p+1}, t_{p+1}\right)$ are isomorphic. In other words, we assume that the variables of the polynomials $y_{\alpha \beta}^{(p)}\left(r_{0}, t_{p}\right)[\theta]$ are the same for all choices of $p$ (since $\theta$ already assigns values to all variables in the zig-zag portion of $B^{(p)}\left(r_{0}, t_{p}\right)$, which depends on $\left.p\right)$.

When there are no migrating variables, then the polynomials $u_{\alpha}^{(0)}, u_{\alpha}^{(1)}, v_{\beta}^{(0)}, v_{\beta}^{(1)}$ in (91) do not contain any variables with $\mathbf{V}^{\prime}$, thus we can drop the argument $\theta$; then we are free to assign their probabilities as we need. However, if a symbol $S^{\prime}$ migrates from right to left, then variables from its equivalence class occur in both $\mathbf{u}_{\alpha}$ and $\mathbf{v}_{\beta}$. The reason is that $\theta$ assigns the same value to all variables in an equivalence class, hence all the tuples $S^{\prime}\left(r_{0}, t_{0}\right), S^{\prime}\left(r_{1}, t_{1}\right), \ldots, S^{\prime}\left(r_{p}, t_{p}\right)$ are associated with the same real variable $s^{\prime}$. Assuming $S^{\prime}$ migrates from right to left, then $S^{\prime}\left(r_{p}, t_{p}\right)$ appears in $v_{\beta}^{(0)}$ (on the "right") and $S^{\prime}\left(r_{0}, t_{0}\right)$ appears in $u_{\alpha}^{(1)}$ (on the "left"). Similarly, $u_{\alpha}^{(0)}$ and $v_{\beta}^{(1)}$ may share common variables. The notation $u_{\alpha}^{(0)}[\theta], \ldots, v_{\beta}^{(1)}[\theta]$ indicates that we apply $\theta$ to all migrating variables $s^{\prime}$, and recall that $\theta\left(s^{\prime}\right)=1 / 2$. Recall that $\theta$ assigns probabilities $1 / 2$ to these variables, hence, we extend it to a complete assignment $\theta^{\prime}$ we must do it consistently with this assignment.

We start by restating the condition $a_{\alpha_{1} \beta_{1}} b_{\alpha_{2} \beta_{2}} \neq a_{\alpha_{2} \beta_{2}} b_{\alpha_{1} \beta_{1}}$.

Lemma C.36. Let $\mathrm{z}$ be a $2 \times 2$ matrix with eigenvalues $0 \neq \lambda_{1} \neq \lambda_{2} \neq 0$, and let $\mathbf{u}_{i}, \mathbf{v}_{i}, i=1,2$ be four vectors. Define the following two sequences, $y_{i}^{(p)}, p \geq 0, i=1,2$ :

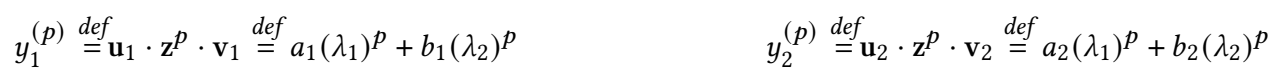

For any $p \geq 0$, consider the following matrix:

$$
\mathbf{D}^{(p)} \stackrel{\text { def }}{=}\left[\begin{array}{cc}
y_{1}^{(p)} & y_{2}^{(p)} \\
y_{1}^{(p+1)} & y_{2}^{(p+1)}
\end{array}\right]
$$

Then the following statements are equivalent:

- $a_{1} b_{2} \neq a_{2} b_{1}$,

- there exists $p \geq 0$ such that $\operatorname{det}\left(\mathbf{D}^{(p)}\right) \neq 0$,

- for all $p \geq 0, \operatorname{det}\left(\mathbf{D}^{(p)}\right) \neq 0$.

Proof. We compute $\operatorname{det}(\mathrm{D})$ directly:

$$
\operatorname{det}\left[\begin{array}{cc}
a_{1} \lambda_{1}^{p}+b_{1} \lambda_{2}^{p} & a_{2} \lambda_{1}^{p}+b_{2} \lambda_{2}^{p} \\
a_{1} \lambda_{1}^{p+1}+b_{1} \lambda_{2}^{p+1} & a_{2} \lambda_{1}^{p+1}+b_{2} \lambda_{2}^{p+1}
\end{array}\right]=\lambda_{1}^{p} \lambda_{2}^{p}\left(\lambda_{2}-\lambda_{1}\right)\left(a_{1} b_{2}-a_{2} b_{1}\right)
$$

and using the fact that $0 \neq \lambda_{1} \neq \lambda_{2} \neq 0$.

Therefore, in order to find an assignment $\theta^{\prime}$ that satisfies $a_{\alpha_{1} \beta_{1}} b_{\alpha_{2} \beta_{2}} \neq a_{\alpha_{2} \beta_{2}} b_{\alpha_{1} \beta_{1}}$, we need to construct $t w o$ blocks, $B^{(p)}\left(r_{0}, t_{p}\right)$ and $B^{(p+1)}\left(r_{0}, t_{p+1}\right): \theta$ already assigns probabilities to the variables in their zig-zag part, hence we only need to extend it with $\theta^{\prime}$ that assigns probabilities to the remaining variables (which we assumed are the same in $B^{(p)}\left(r_{0}, t_{p}\right)$ and $\left.B^{(p+1)}\left(r_{0}, t_{p+1}\right)\right) \operatorname{such}$ that $\operatorname{det}\left(\mathbf{D}_{\alpha_{1} \beta_{1} \alpha_{2} \beta_{2}}^{(p)}\right)\left[\theta^{\prime}\right] \neq 0$, where:

$$
\mathbf{D}_{\alpha_{1} \beta_{1} \alpha_{2} \beta_{2}}^{(p)} \stackrel{\text { def }}{=}\left[\begin{array}{cc}
y_{\alpha_{1} \beta_{1}}^{(p)}[\theta] & y_{\alpha_{2}, \beta_{2}}^{(p)}[\theta] \\
y_{\alpha_{1} \beta_{1}}^{(p+1)}[\theta] & y_{\alpha_{2}, \beta_{2}}^{(p+1)}[\theta]
\end{array}\right]
$$

Furthermore, by lemma C.36 we can check the condition for any $p$, so we choose conveniently to check it for $p=0$, in other words use the blocks $B^{(0)}\left(r_{0}, t_{0}\right)$ and $B^{(1)}\left(r_{0}, t_{1}\right)$. To find $\theta^{\prime}$ such that $\operatorname{det}\left(\mathbf{D}_{\alpha_{1} \beta_{1} \alpha_{2} \beta_{2}}^{(p)}\right)\left[\theta^{\prime}\right] \neq 0$, we proceed as usual: we first prove that the polynomial $\operatorname{det}\left(\mathbf{D}_{\alpha_{1} \beta_{1} \alpha_{2} \beta_{2}}^{(0)}\right) \neq \equiv$, then use this to argue that there exists an assignment $\theta^{\prime}$ of its variables such that $\operatorname{det}\left(\mathbf{D}_{\alpha_{1} \beta_{1} \alpha_{2} \beta_{2}}^{(0)}\left[\theta^{\prime}\right]\right) \neq 0$. 
Lemma C.37. If $\left(\alpha_{1}, \beta_{1}\right) \neq\left(\alpha_{2}, \beta_{2}\right)$, then $\operatorname{det}\left(\mathbf{D}_{\alpha_{1} \beta_{1} \alpha_{2} \beta_{2}}^{(0)}\right) \not \equiv 0$.

Proof. For arbitrary $\alpha, \beta$, denote the following matrices of polynomials (appearing in Eq. (91)):

$$
\mathbf{u}_{\alpha}=\left[\begin{array}{c}
u_{\alpha}^{(0)} \\
u_{\alpha}^{(1)}
\end{array}\right] \quad \mathbf{v}_{\beta}=\left[\begin{array}{c}
v_{\beta}^{(0)} \\
v_{\beta}^{(1)}
\end{array}\right]
$$

Notice that these are polynomials that include the migrating variables. By Eq. (91), we have:

$$
y_{\alpha \beta}^{(0)}=\mathbf{u}_{\alpha}^{T} \mathbf{v}_{\beta} \quad y_{\alpha \beta}^{(1)}=\mathbf{u}_{\alpha}^{T} \mathbf{z v}_{\beta}
$$

For the values $\alpha_{1}, \beta_{1}, \alpha_{2}, \beta_{2}$ given in the lemma, define the following matrix:

$$
\mathbf{D} \stackrel{\text { def }}{=}\left[\begin{array}{cc}
y_{\alpha_{1} \beta_{1}}^{(0)} & y_{\alpha_{2}, \beta_{2}}^{(0)} \\
y_{\alpha_{1} \beta_{1}}^{(1)} & y_{\alpha_{2}, \beta_{2}}^{(1)}
\end{array}\right]=\left[\begin{array}{cc}
\mathbf{u}_{\alpha_{1}}^{T} \mathbf{v}_{\beta_{1}} & \mathbf{u}_{\alpha_{2}}^{T} \mathbf{v}_{\beta_{2}} \\
\mathbf{u}_{\alpha_{1}}^{T} \mathbf{z v}_{\beta_{1}} & \mathbf{u}_{\alpha_{2}}^{T} \mathbf{z v}_{\beta_{2}}
\end{array}\right]
$$

Then $\mathbf{D}[\theta]=\mathbf{D}_{\alpha_{1} \beta_{1} \alpha_{2} \beta_{2}}^{(0)}$, i.e. $\mathbf{D}$ is obtained by exposing the migrating variables, which in $\mathbf{D}_{\alpha_{1} \beta_{1} \alpha_{2} \beta_{2}}^{(0)}$ are assigned by $\theta$ (all are assigned the value $1 / 2)$. Thus, it suffices to prove $\operatorname{det}(\mathbf{D}[\theta]) \not \equiv 0$.

We denote by $\mathrm{T}$ the non-singular matrix that diagonalizes the matrix $\mathrm{z}$, that is $\mathrm{z}=\mathrm{T}^{-1} \Lambda \mathrm{T}$, where $\Lambda=\operatorname{diag}\left(\lambda_{1}, \lambda_{2}\right)$, and define:

$$
\begin{array}{ll}
\mathbf{x}_{\alpha_{1}}^{T} \stackrel{\text { def }}{=} \mathbf{u}_{\alpha_{1}} \mathbf{T} & \mathbf{y}_{\beta_{1}} \stackrel{\text { def }}{=} \mathbf{T}^{-1} \mathbf{v}_{\beta_{1}} \\
\mathbf{x}_{\alpha_{2}}^{T} \stackrel{\text { def }}{=} \mathbf{u}_{\alpha_{2}} \mathbf{T} & \mathbf{y}_{\beta_{2}} \stackrel{\text { def }}{=} \mathbf{T}^{-1} \mathbf{v}_{\beta_{2}}
\end{array}
$$

The matrix $\mathbf{T}$ is a matrix of numbers, while $\mathbf{x}_{\alpha_{1}}, \ldots, \mathbf{y}_{\beta_{2}}$ are vectors of multilinear polynomials. We denote the components of the vector $\mathbf{x}_{\alpha_{1}}$ by $x_{\alpha_{1}}^{(0)}$ and $x_{\alpha_{1}}^{(1)}$, similar to the components of the vector $\mathbf{u}_{\alpha_{1}}$. Notice that $\operatorname{Vars}\left(x_{\alpha_{1}}^{(0)}\right) \subseteq \operatorname{Vars}\left(u_{\alpha_{1}}^{(0)}\right) \cup \operatorname{Vars}\left(u_{\alpha_{1}}^{(1)}\right)$ and $\operatorname{Vars}\left(x_{\alpha_{1}}^{(1)}\right) \subseteq$ $\operatorname{Vars}\left(u_{\alpha_{1}}^{(0)}\right) \cup \operatorname{Vars}\left(u_{\alpha_{1}}^{(1)}\right)$. These variables include the migrating variables. Similarly for the other three vectors. Therefore, we obtain:

$$
\begin{aligned}
\operatorname{det}(\mathbf{D}) & =\left|\begin{array}{cc}
\mathbf{u}_{\alpha_{1}}^{T} \mathbf{v}_{\beta_{1}} & \mathbf{u}_{\alpha_{2}}^{T} \mathbf{v}_{\beta_{2}} \\
\mathbf{u}_{\alpha_{1}}^{T} \mathbf{z} \mathbf{v}_{\beta_{1}} & \mathbf{u}_{\alpha_{2}}^{T} \mathbf{z} \mathbf{v}_{\beta_{2}}
\end{array}\right|=\left|\begin{array}{cc}
\mathbf{x}_{\alpha_{1}}^{T} \mathbf{y}_{\beta_{1}} & \mathbf{x}_{\alpha_{2}}^{T} \mathbf{y}_{\beta_{2}} \\
\mathbf{x}_{\alpha_{1}}^{T} \mathbf{\Lambda}_{\beta_{1}} & \mathbf{x}_{\alpha_{2}}^{T} \Lambda \mathbf{y}_{\beta_{2}}
\end{array}\right| \\
& =\left|\begin{array}{cc}
x_{\alpha_{1}}^{(0)} y_{\beta_{1}}^{(0)}+x_{\alpha_{1}}^{(1)} y_{\beta_{1}}^{(1)} & x_{\alpha_{2}}^{(0)} y_{\beta_{2}}^{(0)}+x_{\alpha_{2}}^{(1)} y_{\beta_{2}}^{(1)} \\
x_{\alpha_{1}}^{(0)} \lambda_{1} y_{\beta_{1}}^{(0)}+x_{\alpha_{1}}^{(1)} \lambda_{2} y_{\beta_{1}}^{(1)} & x_{\alpha_{2}}^{(0)} \lambda_{1} y_{\beta_{2}}^{(0)}+x_{\alpha_{2}}^{(1)} \lambda_{2} y_{\beta_{2}}^{(1)}
\end{array}\right|=\left(x_{\alpha_{1}}^{(0)} y_{\beta_{1}}^{(0)} x_{\alpha_{2}}^{(1)} y_{\beta_{2}}^{(1)}-x_{\alpha_{1}}^{(1)} y_{\beta_{1}}^{(1)} x_{\alpha_{2}}^{(0)} y_{\beta_{2}}^{(0)}\right) \cdot\left(\lambda_{2}-\lambda_{1}\right) \stackrel{\text { def }}{=} f \cdot\left(\lambda_{2}-\lambda_{1}\right)
\end{aligned}
$$

Since $\lambda_{1} \neq \lambda_{2}$, in order to prove $\operatorname{det}(\mathrm{D}[\theta]) \not \equiv 0$, we need to show that $f[\theta] \not \equiv 0$. Assuming the contrary, we have the following identity of polynomials:

$$
\underbrace{x_{\alpha_{1}}^{(0)}[\theta] x_{\alpha_{2}}^{(1)}[\theta]}_{\operatorname{Vars}\left(\mathbf{u}_{\alpha_{1}}[\theta], \mathbf{u}_{\alpha_{2}}[\theta]\right)} \underbrace{y_{\beta_{1}}^{(0)}[\theta] y_{\beta_{2}}^{(1)}[\theta]}_{\operatorname{Vars}\left(\mathbf{v}_{\beta_{1}}[\theta], \mathbf{v}_{\beta_{2}}[\theta]\right)} \equiv \underbrace{x_{\alpha_{1}}^{(1)}[\theta] x_{\alpha_{2}}^{(0)}[\theta]}_{\operatorname{Vars}\left(\mathbf{u}_{\alpha_{1}}[\theta], \mathbf{u}_{\alpha_{2}}[\theta]\right)} \underbrace{y_{\beta_{1}}^{(1)}[\theta] y_{\beta_{2}}^{(0)}[\theta]}_{\operatorname{Vars}\left(\mathbf{v}_{\beta_{1}}[\theta], \mathbf{v}_{\beta_{2}}[\theta]\right)}
$$

We have indicate above the set of variables that occur in these multilinear polynomials. At this point we observe that Vars $\left(\mathbf{u}_{\alpha_{i}}[\theta]\right)$ and $\operatorname{Vars}\left(\mathbf{v}_{\beta_{j}}[\theta]\right)$ are disjoint sets of variables, for any $i, j=1,2$. Indeed, the only variables shared by $\mathbf{u}_{\alpha_{i}}$ and $\mathbf{v}_{\beta_{j}}$ are the migrating variables, but these have been replaced by the constant $1 / 2$ by $\theta$. Therefore, assuming the identity (94) holds, then both the following identities hold too:

$$
x_{\alpha_{1}}^{(0)}[\theta] x_{\alpha_{2}}^{(1)}[\theta] \equiv x_{\alpha_{1}}^{(1)}[\theta] x_{\alpha_{2}}^{(0)}[\theta] \quad y_{\beta_{1}}^{(0)}[\theta] y_{\beta_{2}}^{(1)}[\theta] \equiv y_{\beta_{1}}^{(1)}[\theta] y_{\beta_{2}}^{(0)}[\theta]
$$

Now we return to the definition of the vectors $\mathbf{x}_{\alpha_{i}}, \mathbf{y}_{\beta_{j}}$ in terms of $\mathbf{u}_{\alpha_{i}}, \mathbf{v}_{\beta_{j}}$ and the non-singular matrix $\mathbf{T}$. Assuming the entries in $\mathbf{T}$ are $a, b, c, d$, where $a d-b c \neq 0$, we have:

and we obtain:

$$
\begin{aligned}
& x_{\alpha_{i}}^{(0)}=a u_{\alpha_{i}}^{(0)}+c u_{\alpha_{i}}^{(1)} \\
& x_{\alpha_{i}}^{(1)}=b u_{\alpha_{i}}^{(0)}+d u_{\alpha_{i}}^{(1)}
\end{aligned}
$$

$$
\left(x_{\alpha_{1}}^{(0)} x_{\alpha_{2}}^{(1)}-x_{\alpha_{1}}^{(1)} x_{\alpha_{2}}^{(0)}\right)=\left(\left(a u_{\alpha_{1}}^{(0)}+c u_{\alpha_{1}}^{(1)}\right)\left(b u_{\alpha_{2}}^{(0)}+d u_{\alpha_{2}}^{(1)}\right)-\left(b u_{\alpha_{1}}^{(0)}+d u_{\alpha_{1}}^{(1)}\right)\left(a u_{\alpha_{2}}^{(0)}+c u_{\alpha_{2}}^{(1)}\right)\right)=(a d-b c)\left(u_{\alpha_{1}}^{(0)} u_{\alpha_{2}}^{(1)}-u_{\alpha_{1}}^{(1)} u_{\alpha_{2}}^{(0)}\right)
$$

We apply a similar change of base from $\mathbf{y}_{\beta_{i}}$ to $\mathbf{v}_{\beta_{i}}$, and the identities (95) become:

$$
u_{\alpha_{1}}^{(0)}[\theta] u_{\alpha_{2}}^{(1)}[\theta] \equiv u_{\alpha_{1}}^{(1)}[\theta] u_{\alpha_{2}}^{(0)}[\theta] \quad v_{\beta_{1}}^{(0)}[\theta] v_{\beta_{2}}^{(1)}[\theta] \equiv v_{\beta_{1}}^{(1)}[\theta] v_{\beta_{2}}^{(0)}[\theta]
$$

We have shown that, if $\operatorname{det}(\mathrm{D}[\theta]) \equiv 0$, then both identities (96) hold. We prove that this is a contradiction. For that we show that there exists a total assignment $\theta^{\prime}$ of all variables in the polynomials $\mathbf{u}_{\alpha_{i}}, \mathbf{v}_{\beta_{j}}$ that (1) extends $\theta$, and (2) make at least one of the quantities in (96) $\neq$. 
Notice that when $\alpha_{1}=\alpha_{2}$ then the first identity does hold, but in that case $\beta_{1} \neq \beta_{2}$ and then we show that the second identity implies a contradiction. When both $\alpha_{1}=\alpha_{2}$ and $\beta_{1}=\beta_{2}$ then both identities (96) hold, but we have assumed that $\left(\alpha_{1}, \beta_{1}\right) \neq\left(\alpha_{2}, \beta_{2}\right)$. hold:

To prove our claim, we remove the assignment $\theta$ and start from the polynomials $\mathbf{u}_{\alpha_{i}}, \mathbf{v}_{\beta_{j}}$. We claim that the following non-indentities

$$
\alpha_{1} \neq \alpha_{2} \Rightarrow u_{\alpha_{1}}^{(0)} u_{\alpha_{2}}^{(1)} \not \equiv u_{\alpha_{1}}^{(1)} u_{\alpha_{2}}^{(0)} \quad \beta_{1} \neq \beta_{2} \Rightarrow v_{\beta_{1}}^{(0)} v_{\beta_{2}}^{(1)} \not \equiv v_{\beta_{1}}^{(1)} v_{\beta_{2}}^{(0)}
$$

Indeed, suppose the first identity holds. Then by Theorem B.1 we can factorize the polynomials as follows:

$$
\left[\begin{array}{ll}
u_{\alpha_{1}}^{(0)} & u_{\alpha_{1}}^{(1)} \\
u_{\alpha_{2}}^{(0)} & u_{\alpha_{2}}^{(1)}
\end{array}\right] \equiv\left[\begin{array}{cc}
f \cdot h & f \cdot k \\
g \cdot h & g \cdot k
\end{array}\right]
$$

If $f$ is not a constant polynomial, then $f$ divides both $u_{\alpha_{1}}^{(0)}$ and $u_{\alpha_{1}}^{(1)}$, and therefore it divides $y_{\alpha_{1} \beta_{1}}^{(0)}=u_{\alpha_{1}}^{(0)} v_{\beta_{1}}^{(0)}\left(1-s_{0}\right)+u_{\alpha_{1}}^{(1)} v_{\beta_{1}}^{(1)} s_{0}\left(\right.$ where $s_{0}$ is the articulation variable), which contradicts the fact that $y_{\alpha_{1} \beta_{1}}^{(0)}$ is irreducible. This proves that $f$ must be a constant. Similarly, $g$ must be a constant, by the same argument. It follows that $u_{\alpha_{1}}^{(0)} \equiv c u_{\alpha_{2}}^{(0)}$ for some constant $c$. Since both $u_{\alpha_{1}}^{(0)}$ and $u_{\alpha_{2}}^{(0)}$ are arithmetizations of monotone Boolean functions, when all variables are set to 1 then both quantities are $=1$, hence the constant is $c=1$. Thus, $u_{\alpha_{1}}^{(0)} \equiv u_{\alpha_{2}}^{(0)}$, and by the same argument , $u_{\alpha_{1}}^{(1)} \equiv u_{\alpha_{2}}^{(1)}$ which implies that the two polynomials are identical, $u_{\alpha_{1}} \equiv u_{\alpha_{2}}$, which implies $\alpha_{1}=\alpha_{2}$ by Lemma C.23.

At this point we need to treat separately the cases when $\alpha_{1} \neq \alpha_{2}$ and $\beta_{1} \neq \beta_{2}$ and when one of them is equal. Assume first that both are different, hence both Eq. (97) hold. In that case the following product of polynomials is not identically zero:

$$
f \stackrel{\text { def }}{=}\left(u_{\alpha_{1}}^{(0)} u_{\alpha_{2}}^{(1)}-u_{\alpha_{1}}^{(1)} u_{\alpha_{2}}^{(0)}\right) \cdot\left(v_{\beta_{1}}^{(0)} v_{\beta_{2}}^{(1)}-v_{\beta_{1}}^{(1)} v_{\beta_{2}}^{(0)}\right) \not \equiv 0
$$

We claim that each variable in $f$ has degree $\leq 2$. Indeed, the only variables common in both factors are the migrating variables. Let $s^{\prime}$ be a migrating variable, and assume it migrates from right to left. Then it occurs only in $v_{\beta_{1}}^{(0)}, v_{\beta_{2}}^{(0)}, u_{\alpha_{1}}^{(1)}, u_{\alpha_{2}}^{(1)}$, and therefore it has degree 1 in each of the factors, hence it total degree in $f$ is 2 . Therefore, by Lemma 1.1, there exists an assignment $\theta^{\prime}$ with values in $\{0,1 / 2,1\}$ such that $f\left[\theta^{\prime}\right] \neq 0$. In other words, we have proven that both the following inequalities hold:

$$
u_{\alpha_{1}}^{(0)}\left[\theta^{\prime}\right] u_{\alpha_{2}}^{(1)}\left[\theta^{\prime}\right] \neq u_{\alpha_{1}}^{(1)}\left[\theta^{\prime}\right] u_{\alpha_{2}}^{(0)}\left[\theta^{\prime}\right] \quad v_{\beta_{1}}^{(0)}\left[\theta^{\prime}\right] v_{\beta_{2}}^{(1)}\left[\theta^{\prime}\right] \neq v_{\beta_{1}}^{(1)}\left[\theta^{\prime}\right] v_{\beta_{2}}^{(0)}\left[\theta^{\prime}\right]
$$

It remains to prove that $\theta^{\prime}$ assigns $1 / 2$ to each migrating variable $s^{\prime}$.

Let $s^{\prime}$ be a variable migrating from right to left. We claim that either the following two identities hold:

$$
\begin{aligned}
& u_{\alpha_{1}}^{(0)}\left[s^{\prime}:=0\right] u_{\alpha_{2}}^{(1)}\left[s^{\prime}:=0\right]=u_{\alpha_{1}}^{(1)}\left[s^{\prime}:=0\right] u_{\alpha_{2}}^{(0)}\left[s^{\prime}:=0\right] \\
& v_{\beta_{1}}^{(0)}\left[s^{\prime}:=1\right] v_{\beta_{2}}^{(1)}\left[s^{\prime}:=1\right]=v_{\beta_{1}}^{(1)}\left[s^{\prime}:=1\right] v_{\beta_{2}}^{(0)}\left[s^{\prime}:=1\right]
\end{aligned}
$$

or the following two identities hold (obtained by switching $s^{\prime}:=0$ and $s^{\prime}:=1$ ):

$$
\begin{gathered}
u_{\alpha_{1}}^{(0)}\left[s^{\prime}:=1\right] u_{\alpha_{2}}^{(1)}\left[s^{\prime}:=1\right]=u_{\alpha_{1}}^{(1)}\left[s^{\prime}:=1\right] u_{\alpha_{2}}^{(0)}\left[s^{\prime}:=1\right] \\
v_{\beta_{1}}^{(0)}\left[s^{\prime}:=0\right] v_{\beta_{2}}^{(1)}\left[s^{\prime}:=0\right]=v_{\beta_{1}}^{(1)}\left[s^{\prime}:=0\right] v_{\beta_{2}}^{(0)}\left[s^{\prime}:=0\right]
\end{gathered}
$$

The claim completes the proof, because, assuming the first two equalities hold, (100) and (101), then, if $\theta^{\prime}\left(s^{\prime}\right)=0$ then the left inequality in (99) becomes an equality, while if $\theta^{\prime}\left(s^{\prime}\right)=1$, then the right inequality in (99) becomes an equality, which is a contradiction because we have chosen $\theta^{\prime}$ such that both (99) hold. Similarly for the case when (102) and (103) hold. In either case, $\theta^{\prime}\left(s^{\prime}\right)$ cannot be either 0 or 1 , hence it must be $1 / 2$ and, since $s^{\prime}$ was an arbitrary migrating variable, we have that $\theta^{\prime}$ is an extension of $\theta$.

Thus, it remains to prove the claim. Recall that, for any $\alpha, \beta, y_{\alpha \beta}^{(0)}$ is final, meaning that for every symbol $s^{\prime}$, setting it to 0 or to 1 decomposes the polynomial. ${ }^{8}$ Thus, for any $\alpha, \beta$ :

$$
y_{\alpha \beta}^{(0)}\left[s^{\prime}:=0\right]=a_{\alpha} \cdot b_{\beta}
$$

where $a_{\alpha}$ and $b_{\beta}$ are polynomials that depend only on $\alpha$ and $\beta$ respectively. By assumption, $s_{0}$, causes $s^{\prime}$ to migrate, hence, by Corollary B.16, $s^{\prime}$ will cause $s_{0}$ to migrate. Assume that $s_{0}$ migrates from right to left: that is $s_{0}$ occurs in $b_{\beta}$, and when we decompose $y_{\alpha \beta}^{(0)}\left[s^{\prime}:=1\right]$ then it

${ }^{8}$ There is no need for partial assignment $\theta_{0}$ in Sec. C.7 because when $p=0$ then $\theta_{0}$ is empty; in fact, when $p=0$ then $y_{\alpha \beta}^{(0)}$ is isomorphic to $Q_{\alpha \beta}$, and it is final because $Q$ is final. 
occurs on the left. Then, we apply (104) to $y_{\alpha_{1} \beta}^{(0)}$ and $y_{\alpha_{2} \beta}^{(0)}$ where $\alpha_{1}, \alpha_{2}$ are the values given by the lemma, and $\beta$ is arbitrary, and obtain:

$$
\begin{aligned}
& y_{\alpha_{1} \beta}^{(0)}\left[s^{\prime}:=0, s_{0}:=0\right]=\overbrace{a_{\alpha_{1}} \cdot b_{0}}^{u_{\alpha_{1}}^{(0)}\left[s^{\prime}:=0\right]} \cdot \overbrace{c_{0 \beta}}^{v_{\beta}^{(0)}\left[s^{\prime}:=0\right]} \quad y_{\alpha_{1} \beta}^{(0)}\left[s^{\prime}:=0, s_{0}:=1\right]=\overbrace{a_{\alpha_{1}} \cdot b_{1}}^{u_{\alpha_{1}}^{(1)}\left[s^{\prime}:=0\right]} \cdot \overbrace{c_{1 \beta}}^{v_{\beta}^{(1)}\left[s^{\prime}:=0\right]} \\
& y_{\alpha_{2} \beta}^{(0)}\left[s^{\prime}:=0, s_{0}:=0\right]=\underbrace{a_{\alpha_{2}} \cdot b_{0}} \cdot \underbrace{c_{0 \beta}} \\
& u_{\alpha_{2}}^{(0)}\left[s^{\prime}:=0\right] \quad \underbrace{(0)}_{\beta}\left[s^{\prime}:=0\right] \\
& y_{\alpha_{2} \beta}^{(0)}\left[s^{\prime}:=0, s_{0}:=1\right]=\underbrace{a_{\alpha_{2}} \cdot b_{1}}_{u_{\alpha_{2}}^{(1)}\left[s^{\prime}:=0\right]} \cdot \underbrace{c_{1 \beta}}_{v_{\beta}^{(1)}\left[s^{\prime}:=0\right]}
\end{aligned}
$$

and both sides of (100) become equal to $a_{\alpha_{1}} a_{\alpha_{2}} b_{0} b_{1}$, thus we have proven the identity (100). Applying the same reasoning to the decomposition $y_{\alpha \beta}^{(0)}\left[s^{\prime}:=1\right]$ (where $s_{0}$ occurs on the left) we deduce the identity (101). Thus, when $s_{0}$ migrates from right to left, then both identities (100) and (101) hold. Similarly, when it migrates from right to left then (100) and (101) hold, proving the claim.

Next, assume that $\alpha_{1} \neq \alpha_{2}, \beta_{1}=\beta_{2}$. In that case only the first condition in (97) holds, thus we have:

$$
f \stackrel{\text { def }}{=} u_{\alpha_{1}}^{(0)} u_{\alpha_{2}}^{(1)}-u_{\alpha_{1}}^{(1)} u_{\alpha_{2}}^{(0)} \not \equiv 0
$$

We prove that $f[\theta] \not \equiv 0$, where $\theta\left(s^{\prime}\right)=1 / 2$ for all migrating variables. Here we notice that every migrating variable in $f$ has degree 1 , because it occurs either only in $u_{\alpha_{1}}^{(0)}$ and $u_{\alpha_{2}}^{(0)}$ or only in $u_{\alpha_{1}}^{(1)}$ and $u_{\alpha_{2}}^{(1)}$. We prove that either $f\left[s^{\prime}:=0\right] \equiv 0$ or $f\left[s^{\prime}:=1\right] \equiv 0$. Using the same argument as before, we derive that either (100) holds or (102) holds; equations (101) and (103) hold vacuously because $\beta_{1}=\beta_{2}$. In the first case, when (100) holds, then $f\left[s^{\prime}:=0\right] \equiv 0$; in the second case $f\left[s^{\prime}:=1\right] \equiv 0$. Thus, $f$ is divisible by either $s^{\prime}$ or by $1-s^{\prime}$. It follows that $f$ is a product of the form $s^{\prime}\left(1-s^{\prime \prime}\right) s^{\prime \prime \prime} \ldots$ i.e. there is one factor for each migrating variable $s^{\prime}$, and that factor is either $s^{\prime}$ or $1-s^{\prime}$. It follows that, if $\theta$ assigns values $1 / 2$ to all migrating variables, then $f[\theta] \not \equiv 0$, completing the proof.

Corollary C.38. If $\left(\alpha_{1}, \beta_{1}\right) \neq\left(\alpha_{2}, \beta_{2}\right)$ then there exists an assignment $\theta^{\prime}$ to all variables in $\mathbf{D}_{\alpha_{1} \beta_{1} \alpha_{2} \beta_{2}}^{(0)}$ such that $\operatorname{det}\left(\mathbf{D}_{\alpha_{1} \beta_{1} \alpha_{2} \beta_{2}}^{(0)}\left[\theta^{\prime}\right]\right) \neq 0$. Notice that $\theta^{\prime}$ depends on the choices of $\alpha_{1}, \beta_{1}, \alpha_{2}, \beta_{2}$.

Proof. This is an immediate consequence of the previous Lemma C.37 and of Lemma 1.1, because $\operatorname{det}\left(\mathbf{D}_{\alpha_{1} \beta_{1} \alpha_{2} \beta_{2}}^{(0)}\right)$ is a degree-2 multivariate polynomial that is $\not \equiv 0$.

This completes our goal for this subsection. For any fixed $\left(\alpha_{1}, \beta_{1}\right) \neq\left(\alpha_{2}, \beta_{2}\right)$, we can construct a block $B^{(p)}(u, v)$ isomorphic to $B^{(p+2)}\left(r_{0}, t_{p+2}\right)$ and define an assignment $\theta^{\prime}$ of its variables such that the polynomials $y_{\alpha \beta}^{(p)}(u, v)$ satisfy condition-(80) for the given pair. Importantly, while $\theta^{\prime}$ depends on $\alpha_{1}, \beta_{1}, \alpha_{2}, \beta_{2}$, its restriction to the zig-zag block $B^{(p)}\left(r_{0}, t_{p}\right)$ agrees with $\theta$, and is thus independent on $\alpha_{1}, \beta_{1}, \alpha_{2}, \beta_{2}$, see Fig. 3 (b).

\section{C.10 All Conditions $a_{\alpha_{1} \beta_{1}} b_{\alpha_{2} \beta_{2}} \neq a_{\alpha_{2} \beta_{2}} b_{\alpha_{1} \beta_{1}}$}

In the second part, we show that, if we modify the prefix/suffix blocks by constructing many parallel branches, in order to satisfy condition-(80) for all pairs $\left(\alpha_{1}, \beta_{1}\right) \neq\left(\alpha_{2}, \beta_{2}\right)$.

Consider a block $B^{(p)}(u, v)$ defined by Eq. (90). Let $\mathbf{V}_{\text {pref }}, \mathbf{V}_{\text {suff }}$ be the set of variables (tuples) in the prefix block $B\left(u, r_{0}\right)$ and suffix block $B\left(t_{0}, v\right)$ respectively. These sets will depend on how many branches we choose for these blocks. Let $\mathbf{V}_{\text {zigzag }}$ be the remaining variables, in the zig-zag block $B^{(p)}\left(r_{0}, t_{p}\right)$ and the two remaining sets of dead-end branches at $r_{0}$ and $t_{p}$ respectively. We will fix the following assignment $\theta$ on $\mathbf{V}_{\text {zigzag. }}$. Consider a prefix with a single branch, and a suffix with a single branch, thus $B^{(p)}(u, v)$ is isomorphic to $B^{(p+2)}\left(r_{0}, t_{p+2}\right)$, then apply Corollary C.38. This gives us an assignment $\theta^{\prime}$ to all variables in $B^{(p)}(u, v)$ such that $\operatorname{det}\left(\mathbf{D}_{\alpha_{1} \beta_{1} \alpha_{2} \beta_{2}}^{(p)}\right)\left[\theta^{\prime}\right] \neq 0$, where $\mathbf{D}_{\alpha_{1} \beta_{1} \alpha_{2} \beta_{2}}^{(p)}$ is defined by Eq. (93) w.r.t. the entire block $B^{(p)}(u, v)$. While $\theta^{\prime}$ depends on the choices of $\alpha_{1} \beta_{1} \alpha_{2} \beta_{2}$, its restriction to $B^{(p)}\left(r_{0}, t_{p}\right)$ is independent of $\alpha_{1} \beta_{1} \alpha_{2} \beta_{2}$. Let $\theta$ be that restriction. See Fig. 3 (c) for an illustration.

With the assignment to $\mathbf{V}_{\text {zigzag }}$ fixed, we recompute the probabilities $y_{\alpha \beta}^{(p)}$, by separating the quantities that depend on the prefix/suffix from the rest. For that we use Möbius inversion formula applied to the points $r_{0}$ and $t_{p}$, which separate the two sets of blocks, and obtain:

$$
y_{\alpha \beta}^{(p)}(u, v)=\sum_{\gamma \in L_{0}(\mathbf{G}), \delta \in L_{0}(\mathbf{H})} p_{\alpha \gamma} c_{\gamma \delta}^{(p)} q_{\delta \beta}
$$

where:

$$
p_{\alpha \gamma} \stackrel{\text { def }}{=} \operatorname{Pr}\left(Y_{\alpha \gamma}\left(u, r_{0}\right)\right) \quad c_{\gamma \delta}^{(p)} \stackrel{\text { def }}{=} \mu(\gamma) \mu(\delta) y_{\gamma \delta}^{(p)}\left(r_{0}, t_{p}\right) \prod_{j} y_{\gamma \hat{1}}^{(0)}\left(r_{0}, e_{0}^{(j)}\right) \prod_{j} y_{\hat{1} \delta}^{(0)}\left(f_{0}^{(j)}, t_{0}\right) \quad q_{\delta \beta} \stackrel{\text { def }}{=} \operatorname{Pr}\left(Y_{\delta \beta}\left(t_{p}, v\right)\right)
$$

We think of these quantities as follows. The values $c_{\gamma \delta}$ are constants, since they are defined by the assignment $\theta$ to $\mathrm{V}_{\text {zigzag, and do not }}$ depend on the pair $\left(\alpha_{1}, \beta_{1}\right) \neq\left(\alpha_{2}, \beta_{2}\right)$. The quantities $p_{\alpha \gamma}$ are defined by the prefix block $B\left(u, r_{0}\right)$. The value $p_{\alpha \gamma}$ is defined exactly like $y_{\alpha \beta}$, 
the only difference is that it goes from left-to-left, thus $\alpha, \gamma$ come from the same lattice $L(\mathrm{G})$, with the only restriction that $\gamma \neq \hat{1}$. Similarly, the values $q_{\delta \beta}$ are defined by the suffix block.

We compute $\operatorname{det}\left(\mathbf{D}_{\alpha_{1} \beta_{1} \alpha_{1} \beta_{2}}\right)$ in terms of these new variables $p_{\alpha \gamma}$ and $q_{\delta \beta}$, and denote it by $f_{\alpha_{1} \beta_{1} \alpha_{1} \beta_{2}}$ :

$$
\begin{aligned}
& f_{\alpha_{1} \beta_{1} \alpha_{1} \beta_{2}}=\left|\begin{array}{cc}
y_{\alpha_{1} \beta_{1}}^{(0)} & y_{\alpha_{2}, \beta_{2}}^{(0)} \\
y_{\alpha_{1} \beta_{1}}^{(1)} & y_{\alpha_{2}, \beta_{2}}^{(1)}
\end{array}\right|=\left|\begin{array}{cc}
\sum_{\gamma_{1} \in L_{0}(\mathrm{G}), \delta_{1} \in L_{0}(\mathbf{H})} p_{\alpha_{1} \gamma_{1}} c_{\gamma_{1} \delta_{1}}^{(0)} q_{\delta_{1} \beta_{1}} & \sum_{\gamma_{2} \in L_{0}(\mathrm{G}), \delta_{2} \in L_{0}(\mathbf{H})} p_{\alpha_{2} \gamma_{2}} c_{\gamma_{2} \delta_{2}}^{(0)} q_{\delta_{2} \beta_{2}} \\
\sum_{\gamma_{3} \in L_{0}(\mathbf{G}), \delta_{3} \in L_{0}(\mathbf{H})} p_{\alpha_{1} \gamma_{3}} c_{\gamma_{3} \delta_{3}} q_{\delta_{3} \beta_{1}} & \sum_{\gamma_{4} \in L_{0}(\mathbf{G}), \delta_{4} \in L_{0}(\mathbf{H}) p_{\alpha_{2} \gamma_{4}} c_{\gamma_{4} \delta_{4}}^{(1)} q_{\delta_{4} \beta_{2}}}
\end{array}\right| \\
& =\sum_{\gamma_{1}, \delta_{1}, \ldots, \gamma_{4}, \delta_{4}}\left(c_{\gamma_{1} \delta_{1}}^{(0)} c_{\gamma_{4} \delta_{4}}^{(1)} p_{\alpha_{1} \gamma_{1}} q_{\delta_{1} \beta_{1}} p_{\alpha_{2} \gamma_{4}} q_{\delta_{4} \beta_{2}}-c_{\gamma_{2} \delta_{2}}^{(0)} c_{\gamma_{3} \delta_{3}}^{(1)} p_{\alpha_{1} \gamma_{2}} q_{\delta_{2} \beta_{1}} p_{\alpha_{2} \gamma_{3}} q_{\delta_{3} \beta_{2}}\right) \\
& =\sum_{\gamma_{1}, \delta_{1}, \ldots, \gamma_{4}, \delta_{4}}\left(c_{\gamma_{1} \delta_{1}}^{(0)} c_{\gamma_{4} \delta_{4}}^{(1)} p_{\alpha_{1} \gamma_{1}} q_{\delta_{1} \beta_{1}} p_{\alpha_{2} \gamma_{4}} q_{\delta_{4} \beta_{2}}\right)-\sum_{\gamma_{1}, \delta_{1}, \ldots, \gamma_{4}, \delta_{4}}\left(c_{\gamma_{2} \delta_{2}}^{(0)} c_{\gamma_{3} \delta_{3}}^{(1)} p_{\alpha_{1} \gamma_{2}} q_{\delta_{2} \beta_{1}} p_{\alpha_{2} \gamma_{3}} q_{\delta_{3} \beta_{2}}\right) \\
& =\bar{m} \bar{n} \sum_{\gamma_{1}, \delta_{1}, \gamma_{4}, \delta_{4}}\left(c_{\gamma_{1} \delta_{1}}^{(0)} c_{\gamma_{4} \delta_{4}}^{(1)} p_{\alpha_{1} \gamma_{1}} q_{\delta_{1} \beta_{1}} p_{\alpha_{2} \gamma_{4}} q_{\delta_{4} \beta_{2}}\right)-\bar{m} \bar{n} \sum_{\gamma_{2}, \delta_{2}, \gamma_{3}, \delta_{3}}\left(c_{\gamma_{2} \delta_{2}}^{(0)} c_{\gamma_{3} \delta_{3}}^{(1)} p_{\alpha_{1} \gamma_{2}} q_{\delta_{2} \beta_{1}} p_{\alpha_{2} \gamma_{3}} q_{\delta_{3} \beta_{2}}\right) \\
& =\bar{m} \bar{n} \sum_{\gamma_{1}, \delta_{1}, \gamma_{2}, \delta_{2}}\left(c_{\gamma_{1} \delta_{1}}^{(0)} c_{\gamma_{2} \delta_{2}}^{(1)}-c_{\gamma_{1} \delta_{2}}^{(0)} c_{\gamma_{2} \delta_{1}}^{(1)}\right) p_{\alpha_{1} \gamma_{1}} p_{\alpha_{2} \gamma_{2}} q_{\delta_{1} \beta_{1}} q_{\delta_{2} \beta_{2}} \\
& \stackrel{\text { def }}{=} \bar{m} \bar{n} \sum_{\gamma_{1}, \delta_{1}, \gamma_{2}, \delta_{2}}\left(\Gamma_{\gamma_{1} \gamma_{2} \delta_{1} \delta_{2}}\right) p_{\alpha_{1} \gamma_{1}} p_{\alpha_{2} \gamma_{2}} q_{\delta_{1} \beta_{1}} q_{\delta_{2} \beta_{2}}
\end{aligned}
$$

We make the following observations about this polynomial.

- The quantities $p_{\alpha \gamma}$ and $q_{\delta \beta}$ are defined in (106), and thus depend on the assignment $\theta$ on the variables $\mathbf{V}_{\text {pref }} \cup \mathbf{V}_{\text {suff }}$.

- On the other hand, we can view the quantities $p_{\alpha \gamma}$ and $q_{\delta \beta}$ as independent variables, and thus, $f_{\alpha_{1} \beta_{1} \alpha_{1} \beta_{2}}$ in Eq. (107) is a multivariate polynomial in these variables.

- The polynomial $f_{\alpha_{1} \beta_{1} \alpha_{1} \beta_{2}}$ is not identically 0. This follows from Corollary C.38 since we can construct prefix/suffix blocks consisting of a single branch and extend $\theta$ to a total assignment $\theta^{\prime}$ such that $f_{\alpha_{1} \beta_{1} \alpha_{1} \beta_{2}}=\operatorname{det}\left(\mathbf{D}_{\alpha_{1} \beta_{1} \alpha_{1} \beta_{2}}\right)\left[\theta^{\prime}\right] \neq 0$.

- The coefficients of the polynomial $f_{\alpha_{1} \beta_{1} \alpha_{1} \beta_{2}}$ are $\Gamma_{\gamma_{1} \gamma_{2}} \delta_{1} \delta_{2}$. The total number of variables is $2 \bar{m}+2 \bar{n}$, where $\bar{m}=\left|L_{0}(\mathbf{G})\right|, \bar{n}=\left|L_{0}(\mathbf{H})\right|$.

- When $\alpha_{1}=\alpha_{2}=\alpha, \beta_{1} \neq \beta_{2}$, then the polynomial has only $\bar{m}+2 \bar{n}$ variables, and it simplifies to:

$$
\bar{m} \bar{n} \sum_{\gamma, \delta_{1}, \delta_{2}}\left(c_{\gamma \delta_{1}}^{(0)} c_{\gamma \delta_{2}}^{(1)}-c_{\gamma \delta_{2}}^{(0)} c_{\gamma \delta_{1}}^{(1)}\right) p_{\alpha \gamma}^{2} q_{\delta_{1} \beta_{1}} q_{\delta_{2} \beta_{2}}
$$

The reason is that in Eq. (107), the monomials $p_{\alpha \gamma_{1}} p_{\alpha \gamma_{2}}$ and $p_{\alpha \gamma_{2}} p_{\alpha \gamma_{1}}$ are of course the same, but the coefficients given by $\gamma_{1}, \gamma_{2}$ and $\gamma_{2}, \gamma_{1}$ cancel out, except when $\gamma_{1}=\gamma_{2}$. This polynomial is also not identically zero, $\operatorname{det}\left(\mathbf{D}_{\alpha \beta_{1} \alpha \beta_{2}}\right)\left[\theta^{\prime}\right] \neq 0$, by the same argument.

- Similarly, when $\alpha_{1} \neq \alpha_{2}, \beta_{1}=\beta_{2}=\beta$ the polynomial has only $2 \bar{m}+\bar{n}$ variables, simplifies similarly, and is not $\equiv 0$.

- If both $\alpha_{1}=\alpha_{2}$ and $\beta_{1}=\beta_{2}$, then there are only $\bar{m}+\bar{n}$ variables, but the polynomial is $\equiv 0$. We are not interested in this case.

- Suppose we have built two separate blocks $B_{1}^{(p)}(u, v)$ and $B_{2}^{(p)}(u, v)$, with the same $p$, but different prefixes/suffixes, i.e. different number of parallel branches, and different probability assignments. The values of the quantities (106) are $p_{\alpha \gamma}^{(1)}$ and $q_{\delta \beta}^{(1)}$ in the first block, and $p_{\alpha \gamma}^{(2)}$ and $q_{\delta \beta}^{(2)}$ in the second block; the coefficients $c_{\gamma \delta}^{(p)}$ are the same, since we use the same assignment $\theta$ for the variables $\mathbf{V}_{\text {zigzag }}$ in blocks. Construct a new block $B^{(p)}(u, v)$ whose prefix consists of the union of all parallel branches in $B_{1}^{(p)}(u, v)$ and $B_{2}^{(p)}(u, v)$, and similarly its suffix consists of the union of all parallel branches of the suffices of the two blocks; see Fig. 3 (c). Then the quantities (106) of the new block are given by the products, i.e. $p_{\alpha \gamma}^{(1)} p_{\alpha \gamma}^{(2)}$ and $q_{\delta \beta}^{(1)} q_{\delta \beta}^{(2)}$ respectively. This follows immediately from the fact that, in the Möbius expansion, the formulas for the parallel branches in the suffix/postfix become independent; this is in fact a special case of Theorem C.20.

Next, we state a theorem, of possible independent interest, which we prove in the next section.

Theorem C.39. Let $f_{1}, \ldots, f_{m}$ be multivariate polynomials in the variables $\mathbf{x}=\left(x_{1}, \ldots, x_{n}\right)$. Suppose that, for each $i=1, m$, there exists a set of values $\mathbf{v}_{i}=\left(v_{i 1}, \ldots, v_{i n}\right) \in \mathbb{R}^{n}, v_{i j}>0$, such that $f_{i}\left(\mathbf{v}_{i}\right) \neq 0$. Then there exists natural numbers $k_{1}, k_{2}, \ldots, k_{n} \geq 1$ such that, setting $\mathbf{v}=\left(v_{1}, v_{2}, \ldots, v_{n}\right)$ where $v_{j} \stackrel{d e f}{=} v_{i 1}^{k_{1}} v_{i 2}^{k_{2}} \cdots v_{i n}^{k_{n}}$ we have $f_{1}(\mathbf{v}) \neq 0, \ldots, f_{m}(\mathbf{v}) \neq 0$.

If $\mathbf{v}_{1}, \mathbf{v}_{2}$ are two vectors, then we write $\mathbf{v}_{1} \mathbf{v}_{2}$ for their element-wise product. Thus, the theorem states that, if $f_{i}\left(\mathbf{v}_{i}\right) \neq 0$, then there exists exponents such that, defining $\mathbf{v} \stackrel{\text { def }}{=} \prod_{i} \mathbf{v}_{i}^{k_{i}}$, then for all $i=1, \ldots, m, f_{i}(\mathbf{v}) \neq 0$. We prove the theorem in the next section.

We use this theorem as follows. We need to satisfy several conditions $a_{\alpha_{1} \beta_{1}} b_{\alpha_{2} \beta_{2}} \neq a_{\alpha_{2} \beta_{2}} b_{\alpha_{1} \beta_{1}}$. Let $m$ be the number of such conditions, we will refer to them using an index $i=1,2, \ldots, m$. For each condition $i$ we know that we can satisfy that condition, using a single prefix/suffix branch and some assignment $\theta_{i}$ of the variables $\mathbf{V}_{\text {pref }}, \mathbf{V}_{\text {suff; }}$ t this follows from Corollary C.38. The assignment $\theta_{i}$ results in numerical values $p_{\alpha \gamma}\left[\theta_{i}\right], q_{\delta \beta}\left[\theta_{i}\right]$, and, in particular, on these values, $f_{\alpha_{1} \beta_{1} \alpha_{1} \beta_{2}} \neq 0$. Next, construct parallel branches of the prefix/suffix. In the new block, 
the quantities $p_{\alpha \gamma}$ are products $\prod_{i}\left(p_{\alpha \gamma}\left[\theta_{i}\right]\right)^{k_{i}}$ where $k_{i}$ is the number of copies of the branch with assignment $\theta_{i}$, and similarly for $q_{\delta \beta}$. To apply the Theorem C.39 we need to prove that $p_{\alpha \gamma}\left[\theta_{i}\right]>0$ for all $\alpha, \gamma, \theta_{i}$. Corollary C.38 seems insufficient for that. Instead, we will use the corollary only to prove that the polynomials $f_{\alpha_{1} \beta_{1} \alpha_{1} \beta_{2}}$ are not identically 0 , then prove that we can satisfy each condition $i$ with an assignment that guarantees $p_{\alpha \gamma}\left[\theta_{i}\right]>0$ and $q_{\delta \gamma}\left[\theta_{i}\right]>0$ for all $\alpha, \beta, \gamma, \delta$.

LEMMA C.40. Consider two distinct monomials $p_{\alpha_{1} \gamma_{1}} p_{\alpha_{2} \gamma_{2}} q_{\delta_{1} \beta_{1}} q_{\delta_{2} \beta_{2}}$ and $p_{\alpha_{1} \gamma_{3}} p_{\alpha_{2} \gamma_{4}} q_{\delta_{3} \beta_{1}} q_{\delta_{4} \beta_{2}}$ occurring in the polynomial $f_{\alpha_{1} \alpha_{2} \beta_{1} \beta_{2}}$ (107). Then there exists an assignment $\theta$ to the variables in $\mathbf{V}_{\text {pref }} \cup \mathbf{V}_{\text {suff }}$ such that:

(a) The following inequality holds $p_{\alpha_{1} \gamma_{1}}[\theta] p_{\alpha_{2} \gamma_{2}}[\theta] q_{\delta_{1} \beta_{1}}[\theta] q_{\delta_{2} \beta_{2}}[\theta] \neq p_{\alpha_{1} \gamma_{3}}[\theta] p_{\alpha_{2} \gamma_{4}}[\theta] q_{\delta_{3} \beta_{1}}[\theta] q_{\delta_{4} \beta_{2}}[\theta]$

(b) For every $\alpha, \gamma, p_{\alpha \gamma}[\theta]>0$ and for every $\delta, \beta, q_{\delta \beta}[\theta]>0$.

Proof. We view the two monomials as multilinear polynomials over the variables $\mathbf{V}_{\text {pref }} \cup \mathbf{V}_{\text {suff. }}$ Consider any polynomials $p_{\alpha \gamma}, q_{\delta \beta}$, defined by Eq. (106). The Boolean formula $Y_{\alpha \gamma}$ is similar to $Y_{\alpha \beta}$ in Eq. (66), the only difference is that we are now moving from left to right to left. In particular, $p_{\alpha \gamma}$ is an irreducible polynomial. If $U$ is a left-ubiquitous symbol in $Q$, then we write $U^{\prime}, U^{\prime \prime}$ for the Boolean variables $U^{\prime}=U\left(u, t_{\text {pref }}\right)$ and $U^{\prime \prime}=U\left(r_{0}, t_{\text {pref }}\right)$. We say that $U^{\prime}$ is a ubiquitous variable at the "start" and $U^{\prime \prime}$ is at the "end", since their distance in $Y_{\alpha \gamma}$ is $2 k$. We write $Y_{\alpha \gamma}$ as:

$$
Y_{\alpha \gamma}=G_{\alpha}^{\prime} \wedge Y \wedge G_{\gamma}^{\prime \prime}
$$

where $G_{\alpha}^{\prime}$ consists of clauses that contain some ubiquitous variable $U^{\prime}$ at the start, $G_{\gamma}^{\prime \prime}$ consists of clauses with some ubiquitous variable $U^{\prime \prime}$ at the end, and $Y$ contains all clauses without ubiquitous symbols. Notice that $Y$ is the same formula for all choices of $\alpha$ and $\gamma$.

We will use the following simple fact. If $F$ is a monotone Boolean function in CNF with $n$ variables, and $f$ is its arithmetization, then for any $\theta \in[0,1]^{n}$, if $f[\theta]=0$ then there exists a clause $X_{1} \vee X_{2} \vee \cdots$ in $F$ such that $\theta\left(X_{1}\right)=\theta\left(X_{2}\right)=\ldots=0$. In this case, with some abuse, we write $F[\theta]=0$, although $F[\theta]$ is not a well defined value since $\theta$ assigns real values to Boolean variables.

To prove the lemma, we will prove that there exists an assignment $\theta$ that satisfies item (a) of the lemma, and also satisfies the following properties: (1) $Y[\theta] \neq 0$ (2) for any start- or end-ubiquitous symbol $U, \theta(U) \neq 0$. This implies that, for any $\alpha, \gamma, p_{\alpha \gamma}[\theta] \neq 0$, because the clauses $Y$ will be the same, while each clause in $G_{\alpha}^{\prime}$ or $G_{\gamma}^{\prime \prime}$ has at least one ubiquitous symbol, which is not assigned the value 0 . We enforce similar properties on $Y_{\delta \beta}$, and therefore these imply condition (b) of the lemma.

We start by proving that the two monomials, when viewed as multilinear polynomials over the variables $\mathbf{V}_{\text {pref }} \cup \mathbf{V}_{\text {suff }}$ are not identical polynomials. Suppose otherwise, then:

$$
p_{\alpha_{1} \gamma_{1}} p_{\alpha_{2} \gamma_{2}} q_{\delta_{1} \beta_{1}} q_{\delta_{2} \beta_{2}} \equiv p_{\alpha_{1} \gamma_{3}} p_{\alpha_{2} \gamma_{4}} q_{\delta_{3} \beta_{1}} q_{\delta_{4} \beta_{2}}
$$

Since the polynomials $p_{\alpha \gamma}$ depend only on variables from the prefix while $q_{\delta \beta}$ depend only on variables from the suffix, we obtain that both two identities below must hold:

$$
p_{\alpha_{1} \gamma_{1}} p_{\alpha_{2} \gamma_{2}} \equiv p_{\alpha_{1} \gamma_{3}} p_{\alpha_{2} \gamma_{4}} \quad q_{\delta_{1} \beta_{1}} q_{\delta_{2} \beta_{2}} \equiv q_{\delta_{3} \beta_{1}} q_{\delta_{4} \beta_{2}}
$$

We claim that if the first identity holds, then, when viewed as monomial terms in $f_{\alpha_{1} \beta_{1} \alpha_{2} \beta_{2}}$, the monomials $p_{\alpha_{1} \gamma_{1}} p_{\alpha_{2} \gamma_{2}}$ and $p_{\alpha_{1} \gamma_{3}} p_{\alpha_{2} \gamma_{4}}$ are the same. If the second identity holds as well, then the other two monomials $q_{\delta_{1} \beta_{1}} q_{\delta_{2} \beta_{2}}$ and $q_{\delta_{3} \beta_{1}} q_{\delta_{4} \beta_{2}}$ are also identical, but both cannot hold by the assumption of the lemma. To prove the claim, assume that the first identity above holds. Since each of the four polynomials is irreducible, there are two cases. The first is when $p_{\alpha_{1} \gamma_{1}} \equiv p_{\alpha_{1} \gamma_{3}}$ and $p_{\alpha_{2} \gamma_{2}} \equiv p_{\alpha_{2} \gamma_{4}}$. In that case, using the argument in Lemma C.16, we conclude that $\gamma_{1}=\gamma_{3}$ and $\gamma_{2}=\gamma_{4}$, meaning that monomials $p_{\alpha_{1} \gamma_{1}} p_{\alpha_{2} \gamma_{2}}$ and $p_{\alpha_{1} \gamma_{3}} p_{\alpha_{2} \gamma_{4}}$ are the same. The second case is $p_{\alpha_{1} \gamma_{1}} \equiv p_{\alpha_{2} \gamma_{4}}$ and $p_{\alpha_{2} \gamma_{2}} \equiv p_{\alpha_{1} \gamma_{3}}$, in which case $\alpha_{1}=\alpha_{2}$ and $\gamma_{1}=\gamma_{4}$ and $\gamma_{2}=\gamma_{3}$, and, again, the two monomials are identical.

Therefore, at least one of the identities in (109) does not hold. Assume w.l.o.g. that it is the first identity. We will construct below a certain assignment $\theta$ on $\mathrm{V}_{\text {pref }}$ such that:

$$
p_{\alpha_{1} \gamma_{1}}[\theta] p_{\alpha_{2} \gamma_{2}}[\theta] \neq p_{\alpha_{1} \gamma_{3}}[\theta] p_{\alpha_{2} \gamma_{4}}[\theta]
$$

and satisfies conditions (1) and (2) above. Then we will extend it by setting $\theta(X)=1$ for all variables $X$ in $\mathbf{V}_{\text {suff; }}$ this assignment satisfies both conditions (a) and (b) of the lemma. We distinguish two cases:

Case 1: $\left(\gamma_{1}, \gamma_{2}\right)=\left(\gamma_{4}, \gamma_{3}\right)$. By Lemma 1.1 in the introduction there exists that $\theta \in\{0,1 / 2,1\}$ that satisfies:

$$
p_{\alpha_{1} \gamma_{1}}[\theta] p_{\alpha_{2} \gamma_{2}}[\theta] \neq p_{\alpha_{1} \gamma_{2}}[\theta] p_{\alpha_{2} \gamma_{1}}[\theta]
$$

By Corollary B.3, we may assume w.l.o.g. that neither side $=0$, which implies that $Y[\theta] \neq 0$. We prove that for any ubiquitous symbol $U^{\prime}$ at the start, $\theta\left(U^{\prime}\right) \in\{1 / 2,1\}$. Since the query is final, $Q\left[U^{\prime}:=0\right]$ is a safe query. Since all left clauses remain intact in $Q\left[U^{\prime}:=0\right]$ (none becomes redundant and no subclause becomes redundant), it follows that in $Q_{\text {left }}[U:=0]$ all left clauses are disconnected from the right clauses; referring to a left-to-right path $C_{0}, C_{1}, \ldots, C_{k}$ of minimal length, the clause $C_{1}$ becomes redundant in $Q[U:=0]$. It follows that, for any $\alpha, \gamma$,

$$
Y_{\alpha \gamma}\left[U^{\prime}:=0\right]=G_{\alpha}^{\prime}\left[U^{\prime}:=0\right] \wedge Y_{0} \wedge G_{\gamma}^{\prime \prime} \quad \operatorname{Vars}\left(G_{\alpha}^{\prime}\left[U^{\prime}:=0\right]\right) \cap \operatorname{Vars}\left(Y_{0} \wedge G_{\gamma}^{\prime \prime}\right)=\emptyset
$$

where $Y_{0}$ are clauses of $Y$ that are not redundant in $Y_{\alpha \gamma}\left[U^{\prime}:=0\right]$. Therefore, the polynomials $p_{\alpha \gamma}$ factorize as $p_{\alpha \gamma}\left[U^{\prime}:-0\right]=f_{\alpha} \cdot g_{\gamma}$, and the following equivalence holds $p_{\alpha_{1} \gamma_{1}}\left[U^{\prime}:-0\right] p_{\alpha_{2} \gamma_{2}}\left[U^{\prime}:-0\right] \equiv p_{\alpha_{1} \gamma_{2}}\left[U^{\prime}:-0\right] p_{\alpha_{2} \gamma_{1}}\left[U^{\prime}:-0\right]$. Thus, if $\theta\left(U^{\prime}\right)=0$ then (111) becomes an 
equality, which is a contradiction. If $U^{\prime \prime}$ is a ubiquitous symbol at the end, then we prove similarly that $\theta\left(U^{\prime \prime}\right) \neq 0$. This completes the proof of Case 1 .

Case 2: $\left(\gamma_{1}, \gamma_{2}\right) \neq\left(\gamma_{4}, \gamma_{3}\right)$; by our earlier discussion we also have $\left(\gamma_{1}, \gamma_{2}\right) \neq\left(\gamma_{3}, \gamma_{4}\right)$. Here we will define $\theta\left(U^{\prime}\right)=1$ for all ubiquitous symbols at the start. The four Boolean formulas become:

$$
\begin{array}{ll}
F_{\gamma_{1}} \stackrel{\text { def }}{=} Y_{\alpha_{1} \gamma_{1}}\left[\mathbf{U}^{\prime}:=1\right]=Y \wedge G_{\gamma_{1}}^{\prime \prime} & F_{\gamma_{3}} \stackrel{\text { def }}{=} Y_{\alpha_{1} \gamma_{3}}\left[\mathbf{U}^{\prime}:=1\right]=Y \wedge G_{\gamma_{3}}^{\prime \prime} \\
F_{\gamma_{2}} \stackrel{\text { def }}{=} Y_{\alpha_{2} \gamma_{2}}\left[\mathbf{U}^{\prime}:=1\right]=Y \wedge G_{\gamma_{2}}^{\prime \prime} & F_{\gamma_{4}} \stackrel{\text { def }}{=} Y_{\alpha_{2} \gamma_{4}}\left[\mathbf{U}^{\prime}:=1\right]=Y \wedge G_{\gamma_{4}}^{\prime \prime}
\end{array}
$$

Since all these Boolean formulas are connected, their arithmetizations are irreducible polynomials. It follows that $f_{\gamma_{1}} f_{\gamma_{2}} \not \equiv f_{\gamma_{3}} f_{\gamma_{4}}$, since otherwise we have $\left(\gamma_{1}, \gamma_{2}\right)=\left(\gamma_{3}, \gamma_{4}\right)$ or $\left(\gamma_{1}, \gamma_{2}\right)=\left(\gamma_{4}, \gamma_{3}\right)$. By Lemma 1.1 there exists an assignment $\theta$ in $\{0,1 / 2,1\}$ such that $f_{\gamma_{1}}[\theta] f_{\gamma_{2}}[\theta] \not \equiv f_{\gamma_{3}}[\theta] f_{\gamma_{4}}[\theta]$. We check that these four functions, together with ubiquitous symbols at the end, $U_{1}^{\prime \prime}, \ldots, U_{m}^{\prime \prime}$, satisfy the conditions in Lemma B.5. Indeed, by setting any $U_{i}^{\prime \prime}:=1$, all the subclausess in $G_{\gamma}^{\prime \prime}$ that depend on the choice of $\gamma$ become 1 , since they contain all ubiquitous symbols, and therefore $G_{\gamma_{1}}^{\prime \prime}\left[U_{i}^{\prime \prime}:=1\right] \equiv G_{\gamma_{2}}^{\prime \prime}\left[U_{i}^{\prime \prime}:=1\right] \equiv G_{\gamma_{3}}^{\prime \prime}\left[U_{i}^{\prime \prime}:=1\right] \equiv G_{\gamma_{4}}^{\prime \prime}\left[U_{i}^{\prime \prime}:=1\right]$. Therefore, we can apply Lemma B.5 and obtain an assignment $\theta$ such that $f_{\gamma_{1}}[\theta] f_{\gamma_{2}}[\theta] \not \equiv f_{\gamma_{3}}[\theta] f_{\gamma_{4}}[\theta]$ and $\theta\left(U^{\prime \prime}\right) \in\{1 / 2,1\}$ for all $U^{\prime \prime}$. This concludes the proof.

We now proceed to re-prove Corollary C.38.

Lemma C.41. Fix any $\left(\alpha_{1}, \beta_{1}\right) \neq\left(\alpha_{2}, \beta_{2}\right)$, and consider a prefix/suffix block with a single branch. Then there exists an assignment $\theta$ of the variables in $\mathbf{V}_{\text {pref }} \cup \mathbf{V}_{\text {suff }}$ such that (1) $f_{\alpha_{1}, \beta_{1}, \alpha_{2}, \beta_{2}}[\theta] \neq 0$ and (2) for any $\alpha, \gamma, p_{\alpha \gamma}[\theta]>0$ and for any $\beta, \delta, q_{\delta \beta}[\theta]>0$.

Proof. Denote by $m_{1}, m_{2}, \ldots, m_{n}$ the monomials occurring in all polynomials $f_{\alpha_{1}, \beta_{1}, \alpha_{2}, \beta_{2}}$. That, each $m_{i}$ has the form $m_{i}=p_{\alpha_{1} \gamma_{1}} p_{\alpha_{2} \gamma_{2}} q_{\delta_{1} \beta_{1}} q_{\delta_{2} \beta_{2}}$ for some choice of $\gamma_{1}, \gamma_{2}, \delta_{1}, \delta_{2}$, and we can write $f_{\alpha_{1}, \beta_{1}, \alpha_{2}, \beta_{2}}=\sum_{i} \Gamma_{i} m_{i}$, where $\Gamma_{i}$ is the coefficient of the $i$ 'th monomial. Recall that the polynomial is not identically 0 (this follows from Corollary C.38). For each $i \neq j$, let $\theta_{i j}$ be the assignment given by Lemma C.40 for this pair of monomials. Denote by $\mathbf{p}_{i j}$ be the vector consisting of all values $p_{\alpha \gamma}\left[\theta_{i j}\right]$ and $q_{\delta \beta}\left[\theta_{i j}\right]$ : many do not occur in the polynomial $f_{\alpha_{1}, \beta_{1}, \alpha_{2}, \beta_{2}}$, but we include all of them in these vectors. Notice that all components of all these vectors are $>0$. By Theorem C.39, we can construct prefix/suffix blocks consisting of multiple parallel copies of these blocks, such that (1) the new vectors p are element-wise products of the vectors $\mathbf{p}_{i j}$, and similarly for $\mathbf{q}$; in particular all their components are $>0$, and (2) all pairs of monomials in $f_{\alpha_{1}, \beta_{1}, \alpha_{2}, \beta_{2}}$ are distinct: $m_{i} \neq m_{j}$. Call this block the starting block; notice that it has several parallel branches in both the prefix and the suffix (the same number of branches in the prefix and suffix). Thus, we have:

$$
f_{\alpha_{1}, \beta_{1}, \alpha_{2}, \beta_{2}}(\mathbf{p}, \mathbf{q})=\sum_{i=1, n} \Gamma_{i} m_{i}
$$

Next, we make $k$ parallel copies of the starting block; on this even larger block, the vector $\mathbf{p}$ is replaced by $\mathbf{p}^{k}$, i.e. each component is raised to the power $k$, and similarly for q. Thus, in the new block, the polynomial is:

$$
f_{\alpha_{1}, \beta_{1}, \alpha_{2}, \beta_{2}}\left(\mathbf{p}^{k}, \mathbf{q}^{k}\right)=\sum_{i=1, n} \Gamma_{i} m_{i}^{k}
$$

If this value is $=0$ for all $k=1,2, \ldots, n+1$, then $\Gamma_{1}=\cdots=\Gamma_{n}=0$, because the values $m_{i}$ are distinct and thus the matrix of the system of linear equation is non-singular (it is a Vandermonde matrix). But that implies that the polynomial is identically 0 , which is a contradiction. Thus, there exists $k$ such that the value of this polynomial is $\neq 0$. Since all components of $\mathbf{p}^{k}, \mathbf{q}^{k}$ are $>0$, this proves the claim.

Finally, we pove:

Corollary C.42. There exists a choice of the prefix/suffix blocks such that, for every pair $\left(\alpha_{1}, \beta_{1}\right) \neq\left(\alpha_{2}, \beta_{2}\right)$, the polynomial (107) is $\neq 0$.

Proof. By the previous lemma we can construct a prefix/suffix block that satisfies one polynomial $\neq 0$. By Theorem C.39, we can construct parallel branches of these prefix/suffix blocks to satisfy all polynomials $\neq 0$, as required.

The corollary completes the proof: with this choice of prefix/suffix blocks we have $a_{\alpha_{1} \beta_{1}} b_{\alpha_{2} \beta_{2}} \neq a_{\alpha_{2} \beta_{2}} b_{\alpha_{1} \beta_{1}}$ for all $\left(\alpha_{1}, \beta_{1}\right) \neq\left(\alpha_{2}, \beta_{2}\right)$, and this we proved condition-(80).

It remains to prove Theorem C.39. 


\section{C.11 Proof of Theorem C.39}

Thus, in the rest of this section it remains to prove Theorem C.39. Here we will refer to the polynomials $f_{1}, f_{2}, \ldots$ as $p_{1}, p_{2}, \ldots$

We write a multivariate polynomial as

$$
p\left(x_{1}, \ldots, x_{n}\right)=p(\mathbf{x})=\sum_{\mathbf{e}: \mathbf{e} \leq d} a_{\mathbf{e}} \mathbf{x}^{\mathbf{e}}
$$

Here $\mathbf{e}=\left(e_{1}, \ldots, e_{n}\right) \in \mathbb{N}^{n}$ denotes a vector of exponents, $\mathbf{x} \stackrel{\text { e def }}{=} \prod_{i} x_{i}^{e_{i}}$, and $\mathbf{e} \leq d$ means $e_{i} \leq d$ for all $i$; in other words, each variable $x_{i}$ has degree $\leq d$. We assume that the coefficients $a_{\mathrm{e}}$ are real numbers.

We will consider vectors of values $\mathbf{v}=\left(v_{1}, \ldots, v_{n}\right) \in \mathbb{R}_{+}^{n}$, where $v_{i}>0$ for $i=1, n$. We denote by $\mathbf{v}^{k} \stackrel{\text { def }}{=}\left(v_{1}^{k}, \ldots, v_{n}^{k}\right)$, and denote by vw $\stackrel{\text { def }}{=}\left(v_{1} w_{1}, \ldots, v_{n} w_{n}\right)$, where $\mathbf{w}=\left(w_{1}, \ldots, w_{n}\right)$. Then, Theorem C.39 says that: if $p_{1}\left(\mathbf{v}_{1}\right) \neq 0, \ldots, p_{m}\left(\mathbf{v}_{m}\right) \neq 0$, then there exists $\mathbf{u} \stackrel{\text { def }}{=} \mathbf{v}_{1}^{k_{1}} \cdots \mathbf{v}_{m}^{k_{m}}$ s.t. $p_{1}(\mathbf{u}) \neq 0, \ldots, p_{m}(\mathbf{u}) \neq 0$.

We prove the theorem through a sequence of lemmas. Let $p(\mathbf{x})$ be a multivariate polynomial in $n$ variables, of degree $d$, and let $\mathbf{v} \in \mathbb{R}_{+}^{n}$ be a vector of non-zero values. To compute $p\left(\mathbf{v}^{k}\right)$ we will group the terms of (112) as follows. Let:

$$
U \stackrel{\text { def }}{=}\left\{\mathbf{v}^{\mathbf{e}} \mid \mathbf{e} \leq d\right\} \subseteq \mathbb{R}_{+}
$$

That is, $U$ is the set of all distinct values $\mathbf{v}^{\mathbf{e}}$ that will occur in the expansion of $p(\mathbf{v})$. Assume $U$ has $m$ distinct values, $U=\left\{u_{1}, \ldots, u_{m}\right\}$. For all $u_{i} \in U$, define:

$$
E_{\mathbf{v}, i} \stackrel{\text { def }}{=}\left\{\mathbf{e} \mid \mathbf{e} \leq d, \mathbf{v}^{\mathbf{e}}=u_{i}\right\}
$$

Thus, $E_{\mathbf{v}, 1} \cup E_{\mathbf{v}, 2} \cup \ldots \cup E_{\mathbf{v}, m}$ forms a partition of the set of all exponents occurring in $p(\mathbf{x})$, and we can write it as a sum of $m$ polynomials:

$$
p(\mathbf{x})=\sum_{i=1, m}\left(\sum_{\mathbf{e} \in E_{\mathrm{v}, i}} a_{\mathbf{e}} \mathbf{x}^{\mathbf{e}}\right) \stackrel{\text { def }}{=} \sum_{i=1, m} p_{\mathbf{v}, i}(\mathbf{x})
$$

Then, for all $i, p_{\mathbf{v}, i}\left(\mathbf{v}^{k}\right)=u_{i}^{k} p_{\mathbf{v}, i}(\mathbf{1})$, because all terms $\left(\mathbf{v}^{k}\right)^{\mathbf{e}}$ in $p_{\mathbf{v}, i}\left(\mathbf{v}^{k}\right)$ are equal to $u_{i}^{k}$. Therefore,

$$
p\left(\mathbf{v}^{k}\right)=\sum_{i=1, m} p_{\mathbf{v}, i}\left(\mathbf{v}^{k}\right)=\sum_{i=1, m} u_{i}^{k} p_{\mathbf{v}, i}(\mathbf{1})
$$

Notice that $p_{\mathbf{v}, i}(\mathbf{1})=p_{\mathbf{v}, i}(1,1, \ldots, 1)$ are just the sum of all coefficients of the polynomial $p_{\mathbf{v}, i}(\mathbf{x})$.

Let's call a polynomial $p(\mathbf{x})$ balanced if $p(\mathbf{1})=0$; otherwise it is imbalanced. We prove:

Lemma C.43. Let $\mathbf{v} \in \mathbb{R}_{+}^{n}$ be such that $p(\mathbf{v}) \neq 0$. Then there exists $i$ such that $p_{\mathbf{v}, i}(\mathbf{x})$ is imbalanced.

Proof. We prove the converse: if each $p_{\mathbf{v}, i}(\mathbf{x})$ is balanced, then $p(\mathbf{v})=0$. This follows immediately from Eq. (113).

LEMмa C.44. If at least one of the polynomials $p_{\mathbf{v}, i}(\mathbf{x})$ is imbalanced, then there exists $k_{0} \geq 0$ such that forall $k \geq k_{0}, p\left(\mathbf{v}^{k}\right) \neq 0$. In other words, $p\left(\mathbf{v}^{k}\right) \neq 0$, for all $k$ that are "large enough".

Proof. Assume w.l.o.g. that $m>0$ and all polynomials $p_{\mathbf{v}, i}(\mathbf{x})$ are imbalanced (otherwise we simply remove the balanced polynomials and corresponding values $u_{i}$ ). Also assume $u_{1}>u_{2}>\cdots>u_{m}$. Then Eq. (113) becomes:

$$
p\left(\mathbf{v}^{k}\right)=\sum_{i=1, m} u_{i}^{k} p_{\mathbf{v}, i}(\mathbf{1})=u_{1}^{k}(p_{\mathbf{v}, 1}(\mathbf{1})+\underbrace{\sum_{i=2, m}\left(\frac{u_{i}}{u_{1}}\right)^{k} p_{\mathbf{v}, i}(\mathbf{1})}_{\rightarrow 0})
$$

When $k \rightarrow \infty$, then the expression under $\sum_{i=2, m}$ goes to 0 , hence when $k$ is large enough, $p_{\mathbf{v}, 1}(\mathbf{1})+\sum_{i=2, n}(\cdots) \neq 0$.

For a simple example, consider $f\left(x_{1}, x_{2}, x_{3}\right)=2 x_{1}^{2} x_{2}-x_{3}^{2}$, and assume $\mathbf{v}=(3,2,6)$. Then $f(3,2,6)=2 \cdot 18-36=0$. However, the reader may verify that, for every $k \geq 2, f\left(3^{k}, 2^{k}, 6^{k}\right)>0$.

Next, we will examine combinations of the form $\mathbf{v}^{s} \mathbf{w}^{t}$ for natural numbers $s, t \geq 1$. We will require some simple inequality constraints of these pairs $s, t$, which we define next.

Definition C.45. An inequality constraint is a pair of real numbers $(\alpha, \beta)$, such that $(\alpha, \beta) \neq(0,0)$ (i.e. not both $\alpha, \beta$ can be 0$)$. We say that two natural numbers $s, t \in \mathbb{N}$ satisfy the constraint if $s, t \geq 1$ and $s \alpha+t \beta \neq 0$. If $\Gamma$ is a set of inequality constraints, then we write $\Gamma \models(s, t)$ when $s, t$ satisfy every constraint in $\Gamma$. 
The intuition behind an inequality constraint is the following. We have two numbers $v, w$, and want to find exponents $s, t$ such that $v^{s} \neq w^{t}$. Any pair $(s, t)$ satisfying the constraint $(\log v,-\log w)$ will also satisfy the inequality $v^{s} \neq w^{t}$. We need two very simple facts:

LEMмA C.46. If $\Gamma$ is a finite set of inequality constraints, then there exists infinitely many pairs of natural numbers $s, t$ s.t. $s \geq 1, t \geq 1$ that satisfy all constraints in $\Gamma$.

Proof. Let $\Gamma=\left\{\left(\alpha_{1}, \beta_{1}\right), \ldots,\left(\alpha_{m}, \beta_{m}\right)\right\}$, and define the set $S \stackrel{\text { def }}{=}\left\{-\alpha_{i} / \beta_{i} \mid\left(\alpha_{i}, \beta_{i}\right) \in \Gamma, \beta_{i} \neq 0\right\}$. This is a finite set of real numbers. Then, any pair of natural numbers $s, t$ such that $s, t \geq 1$ and $t / s \in \mathbb{Q}-S$ satisfies all constraints in $\Gamma$ : indeed, if $\beta_{i} \neq 0$ then $t / s \neq-\alpha_{i} / \beta_{i}$ implies $s \alpha_{i}+t \beta_{i} \neq 0$, and if $\beta_{i}=0$ then $s \alpha_{i}+t \beta_{i}=s \alpha_{i} \neq 0$ because $s>0$.

Lemma C.47. Let $\alpha_{1}>\alpha_{2}>\cdots>\alpha_{m}$ and $\beta_{1}>\beta_{2}>\cdots>\beta_{n}$ be two sequences of distinct real values. Then there exists a finite set of inequality constraints $\Gamma$ such that, for any numbers $s, t$, if $\Gamma=(s, t)$, then the $m \cdot n$ values $q \alpha_{i}+t \beta_{j}, i=1, m, j=1, n$, are distinct.

Proof. For all tuples $i_{1}, i_{2}, j_{1}, j_{2}$ such that $1 \leq i_{1} \leq i_{2} \leq m$ and $1 \leq j_{1} \leq j_{2} \leq n$ and $\left(i_{1}, j_{1}\right) \neq\left(i_{2}, j_{2}\right)$, define $\gamma_{i_{1} i_{2} j_{1} j_{2}}=\alpha_{i_{1}}-\alpha_{i_{2}}$ and $\delta_{i_{1} i_{2} j_{1} j_{2}}=\beta_{j_{1}}-\beta_{j_{2}}$. Let $\Gamma$ be the set of constraints $\left(\gamma_{i_{1} i_{2} j_{1} j_{2}}, \delta_{i_{1} i_{2} j_{1} j_{2}}\right)$. If $\Gamma \vDash(s, t)$, then $s \gamma_{i_{1} i_{2} j_{1} j_{2}}+t \delta_{i_{1} i_{2} j_{1} j_{2}} \neq 0$ for all $i_{1}, i_{2}, j_{1}, j_{2}$, which implies $s \alpha_{i_{1}}+t \beta_{j_{1}} \neq s \alpha_{i_{2}}+t \beta_{j_{2}}$.

Let $p(\mathbf{x}, \mathbf{y})=\sum_{\mathbf{e}, \mathbf{f}} a_{\mathbf{e}, \mathbf{f}} \mathbf{x}^{\mathbf{e}} \mathbf{y}^{\mathbf{f}}$ be a polynomial in two sets of variables, and let $\mathbf{v}, \mathbf{w}$ be two sequences of positive real numbers. We define:

$$
\begin{aligned}
U & \stackrel{\text { def }}{=}\left\{\mathbf{v}^{\mathbf{e}} \mid \mathbf{e} \leq d\right\}=\left\{u_{1}>u_{2}>\cdots>u_{m}(>0)\right\} \\
Z & \stackrel{\text { def }}{=}\left\{\mathbf{w}^{\mathbf{e}} \mid \mathbf{e} \leq d\right\}=\left\{z_{1}>z_{2}>\cdots>z_{s}(>0)\right\} \\
E_{\mathbf{v}, \mathbf{w}, i, j} & \stackrel{\text { def }}{=}\left\{(\mathbf{e}, \mathbf{f}) \mid \mathbf{e} \leq d, \mathbf{f} \leq d, \mathbf{v}^{\mathbf{e}}=u_{i}, \mathbf{w}^{\mathbf{f}}=z_{j}\right\}
\end{aligned}
$$

As before, for any two sequences of positive real numbers $\mathbf{v}, \mathbf{w}$, the sets $E_{\mathbf{v}, \mathbf{w}, i, j}$ partition the set of exponents occurring in $p$, and we can write:

$$
p(\mathbf{x}, \mathbf{y})=\sum_{i, j}\left(\sum_{(\mathbf{e}, \mathbf{f}) \in E_{\mathbf{v}, \mathbf{w}, i, j}} a_{\mathbf{e}, \mathbf{f}} \mathbf{x}^{\mathbf{e}} \mathbf{y}^{\mathbf{f}}\right) \stackrel{\text { def }}{=} \sum_{i=1, m ; j=1, s} p_{\mathbf{v}, \mathbf{w}, i, j}(\mathbf{x}, \mathbf{y})
$$

Lemma C.48. Fix $p(\mathbf{x}, \mathbf{y})$, and let $\mathbf{v} \in \mathbb{R}_{+}^{n}$ be a vector of values $>0$, such that $p(\mathbf{v}, \mathbf{1}) \neq 0$. Let $\mathbf{w} \in \mathbb{R}_{+}^{n}$ be any other vector of values $>0$. Then there exists a finite set of inequality constraints $\Gamma$, such that, forall $s, t$, if $\Gamma \vDash(s, t)$ then there exists $k_{0} \geq 0$ such that forall $k \geq k_{0}$, $p\left(\mathbf{u}^{k q}, \mathbf{v}^{k t}\right) \neq 0$.

Proof. Let $U, Z, E_{\mathbf{v}, \mathbf{w}, i, j}, p_{\mathbf{v}, \mathbf{w}, i, j}(\mathbf{x}, \mathbf{y})$ be defined as above. We start by noticing that, for any numbers $a, b \geq 0$, we have $p_{\mathbf{v}, \mathbf{w}, i, j}\left(\mathbf{v}^{a}, \mathbf{w}^{b}\right)=$ $u_{i}^{a} z_{j}^{b} p_{\mathbf{v}, \mathbf{w}, i, j}(\mathbf{1}, \mathbf{1})$. For any three numbers $q, t, k \geq 0$, we have:

$$
\begin{aligned}
p\left(\mathbf{v}^{k q}, \mathbf{w}^{k t}\right) & =\sum_{i=1, m ; j=1, s} p_{\mathbf{v}, \mathbf{w}, i, j}\left(\mathbf{v}^{k q}, \mathbf{w}^{k t}\right) \\
& =\sum_{i=1, m ; j=1, s} u_{i}^{k q} z_{j}^{k t} p_{\mathbf{v}, \mathbf{w}, i, j}(\mathbf{1}, \mathbf{1}) \\
& =\sum_{i=1, m ; j=1, s} r_{i j}^{k} p_{\mathbf{v}, \mathbf{w}, i, j}(\mathbf{1}, \mathbf{1})
\end{aligned}
$$

where $r_{i j} \stackrel{\text { def }}{=} u_{i}^{q} z_{j}^{t}$. We notice that there exists $i, j$ such that $p_{\mathbf{v}, \mathbf{w}, i, j}(\mathbf{1}, \mathbf{1}) \neq 0$. Indeed, if we choose $k=q=1, t=0$, then the quantity above becomes $p\left(\mathbf{v}^{k q}, \mathbf{w}^{k t}\right)=p(\mathbf{v}, \mathbf{1})$, which, by assumption of the lemma is $\neq 0$, proving that at least one quantity $p_{\mathbf{v}, \mathbf{w}, i, j}(\mathbf{1}, \mathbf{1}) \neq 0$.

We will define a set of constraints $\Gamma$ such that $\Gamma \vDash(q, t)$ implies that all values $r_{i j}=u_{i}^{q} z_{j}^{t}$ are distinct or, equivalently, the quantities $q \log u_{i}+t \log z_{j}$ are distinct. To obtain such a $\Gamma$, we apply Lemma C.47 to the sequences $\log u_{i}$ and $\log z_{j}$ respectively. Considering only those values $r_{i j}$ for which $p_{\mathrm{v}, \mathrm{w}, i, j}(\mathbf{1}, \mathbf{1}) \neq 0$, let $r_{i_{0} j_{0}}$ be the largest number. Then we have:

$$
\begin{aligned}
p\left(\mathbf{v}^{k q}, \mathbf{w}^{k t}\right) & =\sum_{i=1, m ; j=1, s} r_{i j}^{k} p_{\mathbf{v}, \mathbf{w}, i, j}(\mathbf{1}, \mathbf{1}) \\
& =r_{i_{0} j_{0}}\left(p_{\mathbf{v}, \mathbf{w}, i_{0}, j_{0}}(\mathbf{1}, \mathbf{1})+\sum_{(i, j) \neq\left(i_{0}, j_{0}\right)}\left(\frac{r_{i j}}{r_{i_{0} j_{0}}}\right)^{k} p_{\mathbf{v}, \mathbf{w}, i, j}(\mathbf{1}, \mathbf{1})\right)
\end{aligned}
$$

Since $\lim _{k \rightarrow \infty}\left(\frac{r_{i j}}{r_{i_{0} j_{0}}}\right)^{k}=0$ it follows that, for $k$ large enough, $p\left(\mathbf{v}^{k q}, \mathbf{w}^{k t}\right) \neq 0$, as required. 
Finally, we can prove Theorem C.39.

Proof. (Of Theorem C.39) We proceed by induction on $m$. When $m=1$ then the theorem holds trivially. Assume $m \geq 2$, and denote $p(\mathbf{x}) \stackrel{\text { def }}{=} p_{1}(\mathbf{x}) \cdot p_{2}(\mathbf{x}) \cdots p_{m-1}(\mathbf{x})$. By induction hypothesis, there exists $\mathbf{v}=\mathbf{v}_{1}^{k_{1}} \cdots \mathbf{v}_{m-1}^{k_{m-1}}$ such that $p(\mathbf{v}) \neq 0$, and there exists $\mathbf{w}$ such that $p_{m}(\mathbf{w}) \neq 0$.

Define the following polynomials $f(\mathbf{x}, \mathbf{y})$ and $g(\mathbf{x}, \mathbf{y})$ :

$$
\begin{aligned}
& f\left(x_{1}, \ldots, x_{n}, y_{1}, \ldots, y_{n}\right) \stackrel{\text { def }}{=} p\left(x_{1} y_{1}, \ldots, x_{n} y_{n}\right) \\
& g\left(x_{1}, \ldots, x_{n}, y_{1}, \ldots, y_{n}\right) \stackrel{\text { def }}{=} p_{m}\left(x_{1} y_{1}, \ldots, x_{n} y_{n}\right)
\end{aligned}
$$

We apply Lemma C.48 to the polynomial $f$ and the sequences $\mathbf{v}$, w: the assumption $f(\mathbf{v}, \mathbf{1}) \neq 0$ holds because $f(\mathbf{v}, \mathbf{1})=p(\mathbf{v}) \neq 0$. Therefore, we obtain a finite set of constraints $\Gamma_{1}$ s.t. for all $s, t$, if $\Gamma_{1}=(s, t)$, then $f\left(\mathbf{v}^{k s}, \mathbf{w}^{k t}\right) \neq 0$ for all $k$ "large enough".

Similarly, we apply Lemma C.48 to the polynomial $g$ and the same sequences $\mathbf{v}, \mathbf{w}$. The condition in the lemma holds, because $g(\mathbf{1}, \mathbf{w})=$ $p_{m}(\mathbf{w}) \neq 0$. Therefore, there exists a finite set of constraints $\Gamma_{2}$ s.t. for all $s, t$, if $\Gamma_{2}=(s, t)$ then $g\left(\mathbf{v}^{k s}, \mathbf{w}^{k t}\right) \neq 0$ for all $k$ "large enough".

Let $(s, t)$ be any pair that satisfies both $\Gamma_{1}$ and $\Gamma_{2}$ : such a pair exists by Lemma C.46, because $\Gamma_{1} \cup \Gamma_{2}$ is a finite set. Therefore, if $k$ is large enough, then, denoting $\mathbf{u} \stackrel{\text { def }}{=} \mathbf{v}^{k s} \mathbf{w}^{k t}$, we notice that this has the required form of the theorem, i.e. $\mathbf{u}=\prod_{i} \mathbf{v}_{i}^{k_{i}}$ for appropriate exponents $k_{i}$, and we prove that it satisfies the conditions of the theorem. Indeed, on one hand $p(\mathbf{u})=p\left(\mathbf{v}^{k s} \mathbf{w}^{k t}\right)=f\left(\mathbf{v}^{k s}, \mathbf{w}^{k t}\right) \neq 0$ which implies $p_{i}(\mathbf{u}) \neq 0$ for all $i=1, m-1$, and on the other hand $p_{m}(\mathbf{u})=p_{m}\left(\mathbf{v}^{k s} \mathbf{w}^{k t}\right)=g\left(\mathbf{v}^{k s}, \mathbf{w}^{k t}\right) \neq 0$, proving the theorem. 